

\section{Editorial Board}

\section{Editor in Chief}

Mark Zilberman, MSc, Shiny World Corporation, Toronto, Canada

\section{Scientific Editorial Board}

Viktor Andrushhenko, PhD, Professor, Academician of the Academy of Pedagogical Sciences of Ukraine, President of the Association of Rectors of pedagogical universities in Europe

John Hodge, MSc, retired, USA

Petr Makuhin, PhD, Associate Professor, Philosophy and Social Communications faculty of Omsk State Technical University, Russia

Miroslav Pardy, PhD, Associate Professor, Department of Physical Electronics, Masaryk University, Brno, Czech Republic

Lyudmila Pet'ko, Executive Editor, PhD, Associate Professor, National Pedagogical Dragomanov University, Kiev, Ukraine

\section{IntellectualArchive, Volume 9, Number 1}

\begin{tabular}{|c|c|}
\hline $\begin{array}{l}\text { Publisher } \\
\text { Address }\end{array}$ & $\begin{array}{l}\text { Shiny World Corp. } \\
9200 \text { Dufferin Street } \\
\text { P.O. Box } 20097 \\
\text { Concord, Ontario } \\
\text { L4K 0C0 } \\
\text { Canada }\end{array}$ \\
\hline $\begin{array}{l}\text { E-mail } \\
\text { Web Site } \\
\text { Series } \\
\text { Frequency } \\
\text { Month } \\
\text { ISSN } \\
\text { DOI } \\
\text { Trademark }\end{array}$ & $\begin{array}{l}\text { : support@IntellectualArchive.com } \\
\text { : www.IntellectualArchive.com } \\
\text { : Journal } \\
\text { : Every } 3 \text { months } \\
\text { : January - March } 2020 \\
: \text { 1929-4700 } \\
: \text { 10.32370/IA_2020_03 } \\
\text { : IntellectualArchiveTM }\end{array}$ \\
\hline
\end{tabular}

(C) 2020 Shiny World Corp. All Rights Reserved. No reproduction allowed without permission. Copyright and moral rights of all articles belong to the individual authors. 


\section{Intellectual Archive}

\section{$\begin{array}{lll}\text { Volume } 9 & \text { Number } 1 & \text { January/March } 2020\end{array}$ \\ Table of Contents}

Physics

M. Pardy

J. C. Hodge

M. Zilberman

L. Koval

Y. Kusparmakov

Y. Kusparmakov

V. Shcherban,

A. Petko,

Ju. Makarenko,

N. Kolva,

D. Egorov

J. Saidov
The elementary particles with imaginary parity

Sgr.A* effect on Mercury and Trans-Neptune Objects

Is Gravity the Only Way to Bend Space-Time?

\section{Engineering}

Simplified Method of Visualization of Light Distribution at the Initial Stage of Design of Symmetrical LED Luminaires

The Principles of the Conceptual Organization and Construction of Patent

Protection

Development of a Patenting and Licensing Strategy

Computer Implementation of the Dichotomy Algorithm for Transcendent Equations

when Determining the Thread Tension

Modern Methods of Processing and Utilization of Oil Sludge

126

\section{Law}

Forensic Examination of Packaging with Indications of Counterfeiting Present on Optical Media in Civil and Criminal Proceedings

Y. Tereshchenko,

H. Strilets

V. Atamanchuk,

N. Pavlovska,

M. Kulyk,

A. Symchuk,

N. Filipova

A. Kapustin
Missed Opportunities for Previous Examinations under the Current Criminal, Civil

Immediate Steps of Judicial Reform in Ukraine 78 


\section{Table of Contents (continued)}

\section{Education}

\section{Y. Laptinova}

A. Yakunina

V. Pererva

I. Khmil

N. Maksimenko

V. Vyshkivska, S. Kushniruk

O. Kalishuk

Unplugging in Language Learning and Teaching

Teaching Fractions in a Course Basic College Mathematics .

165

Terminology Work of Future Biology Teachers During the Field-Based Training in Botany .....

Diagnostics of Formation the Subject Competences in Future Doctors

To the Question of Formation of Competences for the Safety of Life of Students with Intellectual Disabilities

Development of Future Teachers` Professional Thinking by Means of Task

Approach to Organization of Educational Process

Consideration of the Future Music Teacher's Performing Skill in the Aspect of Forming His / Her Performing Mobility

\section{Sport}

T. Salikhov, M. Temperamental Properties of Personality and its Importance in Organization of Shoymardanova Sports Activity

S. Salihov,

Psychological Features of Formation of Emotional-Will Sphere of Athlete

M. Umidjanova

\section{Toronto, January/March 2020}




\title{
The elementary particles with imaginary parity
}

\author{
Miroslav Pardy \\ Department of Physical Electronics \\ Masaryk University \\ Kotlářská 2, 61137 Brno, Czech Republic \\ e-mail:pamir@physics.muni.cz
}

February 1, 2020

\begin{abstract}
The Dirac equation with the imaginary reflection is considered. The intrinsic imaginary parity can be generated in the proton antiproton Dalitz reaction. The violation of the intrinsic parity of an elementary particle can be explained as the oscillation of parity before the particle decay into plus and minus parity system. The explanation is the alternative form of the older explanation.
\end{abstract}

\section{Introduction}

The imaginary parity was postulated by Spitzer $(1960,1963)$, using $K^{0}$ meson. The $K^{0}$ meson has well-defined imaginary parity relative to the vacuum. Further, electromagnetic transitions could carry $K^{0}$ into $\bar{K}^{0}$ in the presence of an external field. A search, by analysing existing experimental results, for the implied dependence of the $K_{1}^{0}-K_{2}^{0}$ mass difference on the external field was proposed and discussed by Natapoff (Natapoff, 1965). However, the mathematical form of the imaginary parity is not realized in the final form. We present the relation of the imaginary parity to the Dirac equation.

Bruno Pontecorvo was the first physicist who introduced the electronneutrino, muon-neutrino and tau-neutrino oscillation. We used here his 
idea to define the so called parity oscillation of $K_{+}, K_{-}$mesons as the analogue of the Pontecorvo model for neutrino oscillations.

\section{The violation of space parity}

It is well known that the parity operator $\mathrm{P}$ acts on the wave function $\psi(x)$ as follows:

$$
P \psi(x)=\lambda \psi(-x), P^{2} \psi(x)=\lambda^{2} \psi(x)=\psi(x),
$$

from which follows that the eigen value of parity $\lambda$ is \pm 1 . The wave function $\psi(x)$ corresponds to the state with positive parity and $-\psi(x)$ corresponds to the state with negative parity.

Now, let us be specific and let us consider the $\theta-\tau$ puzzle. Dalitz (1953; $1956 \mathrm{a} ; 1956 \mathrm{~b})$ analyzed the $\tau$ into three pions and in doing so introduced the Dalitz plot into physics. The first use of the Dalitz plot revealed that the $\theta$ particle appeared to be the same as the $\tau$, which was paradoxical. The puzzle persisted for two years: Dalitz created te hypothesis that perhaps the law of odds and evens was not true, even though all the evidence said otherwise (Dalitz, 1956b).

If the decay is according to form $\theta \rightarrow \pi+\pi$, then parity of $\theta$ is $(-1)(-1)=1$, and if $\tau \rightarrow \pi+\pi+\pi$, then parity of $\tau$ is $(-1)(-1)(-1)=-1$. However, it was experimentally confirmed that $\theta$ and $\tau$ are the same particle called K-meson. So we have two decays:

$$
K \rightarrow \pi+\pi
$$

and

$$
K \rightarrow \pi+\pi+\pi
$$

The first process can be evidently described by Hamiltonian $H_{1}=A B_{+}$, and the second process can be described by Hamiltonian $H_{2}=A B_{-}$, while the original Hamiltonian is

$$
H=A\left(C_{1} B_{+}+C_{2} B_{-}\right),
$$

where $C_{1}, C_{2}$ are some constants.

After application o the parity operator on the Hamiltonian (4), we get

$$
P H=A\left(C_{1} B_{+}-C_{2} B_{-}\right)
$$


It is possible to show, (Lee, et al., 1956; Yang, 1964), that the angular distribution $N(\varphi)$ of electrons in beta decay of cobalt $C_{60}$ is described by function, which follows from the Hamiltonian, which is combination of positive parity term and negative parity term (4). The resulting function is

$$
N(\varphi)=A(1+a \cos \varphi) .
$$

It means that the correct description of the process of the violation of parity is described by the Hamiltonian with the positive and negative parity term.

\section{The oscillation of parity}

The main idea of this section is in the following principle: If system $K$ is described by Hamiltonian $H_{1}=A B_{+}$, where $A, B_{+}$are the positive parity operators, and at the same time it can be described by the Hamiltonian $H_{2}=A B_{-}$, where $B_{-}$is the negative parity operator, then it can be in reality described by the Hamiltonian $H=A\left(C_{1} B_{+}+C_{2} B_{-}\right)$.

The immediately statement following from the last principle is, that $K$ system oscillates between positive and negative parity states to form the positive parity states, or the negative parity states. If the system is $K$ meson, then this meson oscillates between positive and negative mesons, forming $\theta$-particle and $\tau$-particle.

The oscillation of parity is the crucial problem of the future particle physics. It is not confirmed that the oscillation of parity is equivalent to the violation of parity. We write it as follows

$$
\begin{gathered}
\theta(t)=\tau(t) \rightarrow(\pi+\pi) ; \quad P=1 \\
\theta(t+\Delta t)=\tau(t+\Delta t) \rightarrow(\pi+\pi+\pi) ; P=-1
\end{gathered}
$$

The fall of parity open the way for a reconsideration of physical theories and led to new, discoveries regarding the nature of matter and the universe. The work of Lee and Yang (1956), Wu et al. (1957), and Garwin et al. (1957) has provided definitive proof that mirror symmetry is broken in the weak interaction, and it is not a true symmetry of the Universe as a whole. This is an surprising conclusion, because the historical trend of physics up to this point was that of simplification and unification. In the case of parity violation, however, we learned that nature exhibits a very striking asymmetry for no apparent reason, and goes against our expectation of a symmetrical universe. 
The matrix element describing the violation of parity during the $\beta-$ decay of neutron into the electron and electron antineutrino is of the form (Commins et al., 1983):

$$
M=\frac{G_{F}}{\sqrt{2}} \int d \mathbf{x} \bar{\psi}_{p} \gamma_{\mu}\left(1-\lambda \gamma_{5}\right) \psi_{n} \psi_{e} \gamma^{\mu}\left(1-\gamma_{5}\right) \psi_{\bar{\nu}_{e}} ; \quad \lambda \approx 1,25,
$$

where the asymetry of the matrix element $M$ is caused by therm with $\gamma^{5}$, where parity of $\bar{\psi} \gamma^{\mu} \gamma^{5} \psi$ is $-(-1)^{\mu} ; \quad \gamma^{5}=\gamma^{1} \gamma^{2} \gamma^{3} \gamma^{4} ; \mu=0,1,2,3$ (Peskin et al., 1977). The analogical matrix element was derived for the decay of the $\mu$ meson and so on (Commins et al., 1983).

\section{The imaginary parity}

The imaginary parity operator $\Pi$ acts on the wave function $\psi(x)$ as follows:

$$
\begin{gathered}
\Pi \psi(x)=\Lambda \psi(i x), \Pi^{2} \psi(x)=\Lambda^{2} \psi(-x), \\
\Pi^{3} \psi(x)=\Lambda^{3} \psi(-i x), \Pi^{4} \psi(x)=\Lambda^{4} \psi(x)=\psi(x)
\end{gathered}
$$

from which follows that the eigen value of parity equation $\Lambda^{4}=1$ is \pm 1 and $\pm i$ The wave function $+\psi(x)$ corresponds to the state with the positive parity and $-\psi(x)$ corresponds to the state with negative parity. The wave function $+\mathrm{i} \psi(x)$ corresponds to the state with the positive imaginary parity and $-i \psi(x)$ corresponds to the state with the negative imaginary parity. While the algebra of the states of the imaginary parity is elementary, the experimental proof of the existence of the particles in the states of the imaginary parity is sophisticated.

Let us consider the following particle reaction to establish the imaginary parity (Dalitz, 1962).

$$
(\bar{p}+p) \rightarrow \pi+\pi+\pi+\pi+\pi^{0},
$$

where the particle $\pi^{0}$ can be detected by the Dalitz method (Dalitz, 1962). The parity of the right side of eq. $(10)$ is $(-1) \times(-1) \times(-1) \times(-1) \times(-1)=$ $(-1)$. So, the parity of the left side is also $(-1)$. However the process $(\bar{p}+p)$ involves the possible channel with particles in the states with the imaginary parity leading to the conservation of parity. Namely, $(i) \times(i) \times(i) \times(i) \times(-1)=(-1)$, where only $\pi^{0}$-meson was not replaced by $i$. 
The similar reactions and decay channels having the imaginary parity are involved in the Particle Data Group (Tanabashi et al., 2018) and can be verified by the well-educated experimenters.

\section{The intrinsic parity and the pion}

Let us consider the parity of the $\pi^{-}$meson (Muirhed, 1968). When $\pi^{-}$ mesons are brought to rest in liquid hydrogen or deuterium, then the following reactions are observed (Panovsky, Aamodt and Hadley, 1951):

$$
\begin{aligned}
& \pi^{-}+p \rightarrow n+\gamma, \\
& \pi^{-}+p \rightarrow n+\pi^{0}
\end{aligned}
$$

and

$$
\begin{gathered}
\pi^{-}+d \rightarrow n+n, \\
\pi^{-}+d \rightarrow n+n+\gamma .
\end{gathered}
$$

The surprising feature of these transitions is the absence of $\pi^{0}$ mesons from the reactions in deuterium, whereas in hydrogen one-half of the reactions produce $\pi^{0}$ mesons. This result may be understand if the $\pi^{-}$ meson has odd parity.

Another demonstration of the pion parity was given in 1954, by Chinowsky and Steinberger (Chinowsky et al., 1954). They studied the decay of an "atom" made from a deuteron $\left({ }_{1}^{2} \mathrm{H}^{+}\right)$and a negatively charged pion $\pi^{-}$in a state with zero orbital angular momentum $l=0$ into two neutrons $n$.

So, we see that parity is not defined by the microscopic theory of the electron structure but only by phenomenology. Let us remark that it is well known that $C P$ parity is violated and it is not excluded that the analogical violation is valid for $C \Pi$ parity being the crucial problem for the well educated experimenters in particle physics.

\section{The parity of the wave functions}

Now, let us still remember the mathematical definition of parity in quantum mechanics. So, let us consider the relative motion of two particle in 
quantum theory. The wave function of such two-body system is described by the wave function

$$
\psi_{l}(r)=R(l) P_{l}^{m}(\cos \theta) e^{i m \varphi},
$$

where $P_{l}^{m}(\cos \theta)$ is the associate Legendre function. After the operation of reflection with $(\cos \theta) \rightarrow(-\cos \theta)$, the Legendre coefficient is transformed to $(-1)^{l-m}$, where $l$ is the quantum number of the orbital momentum with projection on the polar axis. During reflection $r \rightarrow r$, and $\theta \rightarrow \pi-\theta$, we have $\cos (\pi-\theta) \rightarrow-\cos \theta, \varphi \rightarrow \pi+\varphi$, and $e^{i m \varphi} \rightarrow e^{i m(\pi+\varphi)} \rightarrow(-1)^{m} e^{i m \varphi}$. Then,

$$
\begin{gathered}
\psi_{l}(r)=R(l) P_{l}^{m}(\cos \theta) e^{i m \varphi} \rightarrow \\
R(l)(-1)^{l-m} P_{l}^{m}(\cos \theta) e^{i m \varphi}(-1)^{m}=(-1)^{l} \psi_{l}(r)
\end{gathered}
$$

So, the parity of the system is

$$
P_{l}=(-1)^{l}
$$

\section{The imaginary parity of the plane wave}

If we apply the imaginary reflection $\mathbf{x} \rightarrow i \mathbf{x}$ on the particle in the state of the plane wave

$$
\psi(\mathbf{x}, t)=e^{i(\mathbf{k x}-\omega t)}
$$

we get

$$
\psi(\mathbf{x}, t) \rightarrow e^{-\mathbf{k x}-i \omega t},
$$

which means that the elementary particle is in the nonperiodic space state and the imaginary parity cannot be detected by the Dalitz method.

The mechanism of the imaginary reflection is not known similarly to the mechanism of the normal reflection. One possibility is the oscillation of parity of the original particle, or of the original process.

\section{Parity and the Dirac equation}

The transformation of the mirror reflection is

$$
\mathbf{x} \rightarrow-\mathbf{x} ; \quad t \rightarrow t
$$


and the corresponding matrix of transformation is is:

$$
a_{\alpha \lambda}=\left(\begin{array}{cccc}
-1 & 0 & 0 & 0 \\
0 & -1 & 0 & 0 \\
0 & 0 & -1 & 0 \\
0 & 0 & 0 & 1
\end{array}\right)
$$

The invariance of the Dirac equation with regard to transformation (19) generates the following equation involving the transformation matrix $S$ (Muirhead, 1968):

$$
\gamma_{\alpha}=a_{\alpha \lambda} S \gamma_{\lambda} S^{-1}
$$

which can be rewriting in the explicit form as follows:

$$
\begin{aligned}
& \gamma_{1}=-S \gamma_{1} S^{-1}, \gamma_{2}=-S \gamma_{2} S^{-1} \\
& \gamma_{3}=-S \gamma_{3} S^{-1}, \gamma_{4}=+S \gamma_{4} S^{-1}
\end{aligned}
$$

with the solution

$$
S=\gamma_{4}
$$

\section{Imaginary parity and the Dirac equation}

In this case the metric tensor is of the form

$$
a_{\alpha \lambda}=\left(\begin{array}{cccc}
i & 0 & 0 & 0 \\
0 & i & 0 & 0 \\
0 & 0 & i & 0 \\
0 & 0 & 0 & 1
\end{array}\right)
$$

and we perform the operation by the analogy with the previous section. So, the invariance of the Dirac equation with regard to transformation (23) generates the following equation involving the transformation matrix $S$ :

$$
\gamma_{\alpha}=a_{\alpha \lambda} S \gamma_{\lambda} S^{-1}
$$

which can be rewriting in the explicit form as follows:

$$
\begin{gathered}
\gamma_{1}=i S \gamma_{1} S^{-1}, \gamma_{2}=i S \gamma_{2} S^{-1} \\
\gamma_{3}=i S \gamma_{3} S^{-1}, \quad \gamma_{4}=S \gamma_{4} S^{-1} .
\end{gathered}
$$


with the solution of $\gamma_{4}=S \gamma_{4} S^{-1}$

$$
S=\gamma_{4}
$$

which is not solution for eqs. with $\gamma_{1}, \gamma_{2}, \gamma_{3}$ in eq. (25).

\section{The space-time metric in the imaginary mirror}

Let us consider the Minkowski space-time metric:

$$
d s^{2}=c^{2} d t^{2}-d x^{2}-d y^{2}-d z^{2} .
$$

Then, this metric in the imaginary mirror is of the form:

$$
d s^{2}=c^{2} d t^{2}+d x^{2}+d y^{2}+d z^{2} .
$$

Now, let us consider relativistic spherical metric:

$$
d s^{2}=c^{2} d t^{2}-d r^{2}-r^{2} d \theta^{2}-r^{2} \sin ^{2} \theta d \varphi^{2} .
$$

Then, in the imaginary mirror, the metric i as follows $(r \rightarrow i r)$

$$
d s^{2}=c^{2} d t^{2}+d r^{2}+r^{2} d \theta^{2}+r^{2} \sin ^{2} \theta d \varphi^{2} .
$$

\section{Discussion}

To probe the spin and parity quantum numbers of the discovered particles, a systematic analysis of its production and decay processes is necessary to perform. An excess over the expected background must be used to further discriminate between signal hypotheses. These analysis are based on probing various alternative models of spin and parity.

The models can be expressed in terms of an effective Lagrangian, or, in terms of helicity amplitudes. The two approaches are equivalent. The effective Lagrangian formalism is frequently chosen to describe the models considered and a restricted number of models are discussed.

In the analysis performed by CMS a larger number of models have been investigated, however the main channels studied by both experiments are essentially the same and the main conclusions are similar and fully consistent (Tanabashi et al., 2018).

The revision of all experiments in the Tanabashi monograph (Tanabashi et al., 2018) can lead to the discovery of the imaginary parities of elementary particles and to the new deal of physics, while the parities of black 
hole, white hole and wormhole hole follow from the Einstein equations. On the other hand the parity of the black hole as the black body (Lee, 1988) is the parity of the black body photons with the parity of every photon $(-1)$ (Berestetzkii, et al., 1989).

The operation of parity can also be expressed as a rotation of the decay plane, so no parity violating kinematic observables can be defined (unless they also violate rotational invariance). The use of parity-odd observables in four body decays is discussed in literature (Tanabashi et al., 2018).

\section{References}

Berestetzkii, V. B., Lifshitz, E. M. and Pitaevskii, L. P. Quantum Electrodynamics, (Moscow, Nauka, 1989). (in Russian).

Chinowsky, W. and Steinberger, J. (1954). Absorption of negative pions in deuterium: parity of the pion. Physical Review 95 (6), 1561-1564.

Commins, E. D and Bucksbaum, P. H. Weak interactions of leptons and quarks, (Cambridge University Press, 1983)

Dalitz, R. H. Strange Particles and Strong Interactions, (Oxford University Press, 1962).

Dalitz, R. H. (1953). On the analysis of $\tau$-meson data and the nature of the $\pi$-meson, Philosophical Magazine 44, 1068).

Dalitz, R. H. (1956a). Isotopic spin changes in $\tau$ and $\theta$ decays, Proc. Phys. Soc. A69, 527.

Dalitz, R. H. (1956b). Present status of $\tau$ Spin-Parity, High Energy Physics, Proceedings of the Sixth Annual Rochester conference, April 37, edited by J. Ballam, V.L. Fitch, T. Fulton, K., Huanf, R. R. Rau, S. B. and Treiman, P. VIII-19, Interscience Publishers Inc., New York. 67053-5.

Garwin, R. L., Lederman, L. M. and Weinrich, M. (1957). Observations of the failure of conservation of parity and charge conjugation in meson decays: The magnetic moment of the free muon. Physical Review 105 (4), $1415-1417$.

Lee, T. D., Yang, C. N. (1956). Question of parity conservation in weak interactions. Physical Review 104 (1), 254-258. 
Lee, T. D. (1988). Do black holes emit black-body radiation? (Preprint of Columbia University).

Muirhead, H. The Physics of Elementary Particles, (Pergamon Press, Oxford, London et al., second ed. 1968).

Natapoff, A. (1965). Is the parity the $K^{0}$ meson imaginary?, Nuclear Physics, 66, p. 653-656.

Panofsky, W. K. H., Aamodt, R. L. and Hadley, J. (1951). The gammaray spectrum resulting from capture of negative $\pi$-mesons in hydrogen and deuterium, Phys. Rev. 81, 565.

Peskin, M. E. and Schroeder, D. V. An Introduction to quantum Field Theory, (Addison-Wesley Publishing Company, Reading, Massachusetts, Menlo Park CA, New York, USA, 1997).

Spitzer, R. (1960). On neutral spin-0 particles with imaginary parity , Nuclear Physics, 21, 681-685.

Spitzer, R. (1963). The parity of the neutral K meson, Nuclear Physics, 47, 367-384.

Tanabashi, T. et al. (2018). Particle Data Group, Phys. Rev. D 98, 030001

Yang, C. N. (1964). The law of parity conservation and other symmetry laws of physics, Nobel Lectures Physics, 1942-1962,

Wu, C. S., Ambler, E, Hayward, R. W., Hoppes, D.D. and Hudson, R. P. (1957). Experimental test of parity conservation in beta decay. Physical Review 105 (4), 1413-1415. 


\title{
Sgr.A* effect on Mercury and Trans-Neptune Objects
}

\author{
J.C. Hodge $\mathrm{e}^{1 *}$ \\ ${ }^{1}$ Retired, 477 Mincey Rd., Franklin, NC, 28734
}

\begin{abstract}
The Scalar Theory of Everything (STOE) has suggested the plenum from the Source of the Milky-Way galaxy (Galaxy) causes the observed rotation curves of galaxies and is the cause of the "Planet 9" orbits of icy objects. A correlation of the apsidal lines of the rocky planets is also consistent with their being a plenum caused force influencing the orbits. The study of planetary objects should include a parameter reflecting the effect of the STOE Source.
\end{abstract}

The orbit of Mercury is eccentric and inclined relative to other planets. The orbits of Trans-Neptune Objects (TNOs) have also been measured to be eccentric and highly inclined to the plane of the solar system. The Scalar Theory Of Everything proposed a new physics explanation of the TNOs to replace the "Planet 9" traditional explanation (Hodge 2019). This explanation was predicted by the explanation of the Pioneer Anomaly (Hodge 2006)

(Bartlett 2020) suggested the perihelion of Mercury resembles the orbits of the TNOs. Therefore, the orbit of Mercury should have a relation to the Scalar Theory of Everything (STOE) Source (Sagittarius A*). This is surprising for the STOE because the inner planets are rocky objects and the TNOs are icy-with-methane objects. The STOE suggests the divergence of the plenum (hence force) from the Source at Sagittarius A* (Sgr A*) acts on the surface area (a radius ${ }^{-2}$ relation). Gravity acts on the mass (radius ${ }^{-3}$ relation). The difference causes the decreasing metallicity with increasing Galactic radius relation, pushing lighter elements outward.

\footnotetext{
*E-mail: jchodge@frontier.com
} 
This paper examines the data.

Table 1 Shows the aphelion (Ap.)and perihelion (Peri.) of the rocky planets and two of the more extreme TNOs. The two TNOs listed represent the RA extremes.

The last line shows the position of Sgr A* in the Ap. column. The Source pushes matter from it to the coordinates in the Peri. column. Therefore, the Source effect is to cause a perihelion on objects in the listed perihelion direction.

A tendency of solar system objects is to align their apsidal lines and, also, their orbital planes with the STOE plenum force. Mercury is the closest aligned of the objects listed. Venus and Earth also show a tendency to align. Mars seems the outlier.

The solar system is revolving around the Galaxy center. This causes a change in the relative direction of the Source force of $\approx 0.014^{\circ} /$ year. This change in direction could cause the objects to deviate inclination of the plane of the solar system. Such change would be more pronounced in icy objects than in rocky objects. This is measured in TNOs. Those objects with very long rotation periods would experience a greater changing direction of the force. This would present a very confusing orbit to gravitational only orbit calculations as some of the simulations show.

This further suggests the TNOs are being pushed out of the solar system on orbits becoming hyperbolic.

This force would also lift the perihelia of long-period Kuiper Belt Objects (KBOs) as their orbit data shows that explain the origin of the Neptunedetached KBOs. At the same time, Oort cloud objects traveling toward Sgr $A^{*}$ have their orbits reduced. This, also, could be a source of long-period KBOs.

Because the TNOs are icy objects and they venture far from the gravity of the Sun, the effect of the STOE Source is more pronounced. Perhaps, the simulations of the TNOs orbits would be more accurate with consideration of the Source.

The experience of the long, fruitless search for Dark Matter and the search for Vulcan suggest new physics should be considered.

The study of planetary objects should include a parameter reflecting the effect of the STOE Source. Perhaps one of the PPN factors should be this force. 
Table 1: Aphelion and perihelion of some solar system objects. ${ }^{\text {a }}$

\begin{tabular}{|c|c|c|c|c|c|c|}
\hline Object & $\begin{array}{l}\text { Ap. } \\
\text { RA } \\
\text { hours }\end{array}$ & $\begin{array}{l}\text { DEC } \\
\text { deg. }\end{array}$ & $\begin{array}{l}\text { Dist. }^{c} \\
\text { AU }\end{array}$ & $\begin{array}{l}\text { Peri. }^{b} \\
\text { RA } \\
\text { hours }\end{array}$ & $\begin{array}{l}\text { DEC } \\
\text { deg. }\end{array}$ & $\begin{array}{l}\text { Dist. }^{c} \\
\text { AU }\end{array}$ \\
\hline Mercury $^{\mathrm{d}}$ & $17^{\mathrm{h}} 03^{\mathrm{m}}$ & $-26^{\mathrm{o}} 14^{\mathrm{m}}$ & 0.47 & $05^{\mathrm{h}} 03^{\mathrm{m}}$ & $+26^{\mathrm{o}} 14^{\mathrm{m}}$ & 0.31 \\
\hline Venus ${ }^{\mathrm{e}}$ & $20^{\mathrm{h}} 58^{\mathrm{m}}$ & $-20^{\mathrm{o}} 02^{\mathrm{m}}$ & 0.73 & $08^{\mathrm{h}} 59^{\mathrm{m}}$ & $+19^{\mathrm{o}} 58^{\mathrm{m}}$ & 0.72 \\
\hline $\operatorname{Earth}^{\mathrm{f}}$ & $18^{\mathrm{h}} 51^{\mathrm{m}}$ & $-22^{\mathrm{o}} 55^{\mathrm{m}}$ & 1.02 & $07^{\mathrm{h}} 02^{\mathrm{m}}$ & $+22^{\mathrm{o}} 41^{\mathrm{m}}$ & 0.98 \\
\hline Mars $^{g}$ & $10^{\mathrm{h}} 34^{\mathrm{m}}$ & $+10^{\mathrm{o}} 54^{\mathrm{m}}$ & 1.67 & $22^{\mathrm{h}} 34^{\mathrm{m}}$ & $-10^{\mathrm{o}} 53^{\mathrm{m}}$ & 1.38 \\
\hline TNOs & & & & & & \\
\hline Sedna ${ }^{\mathrm{h}}$ & & $?$ & & $06^{\mathrm{h}} 27^{\mathrm{m}}$ & $+14^{\mathrm{o}} 23^{\mathrm{m}}$ & 76.2 \\
\hline $2007 \mathrm{TG} 422^{\mathrm{i}}$ & & $?$ & & $02^{\mathrm{h}} 51^{\mathrm{m}}$ & $-02^{\mathrm{o}} 21^{\mathrm{m}}$ & 35.6 \\
\hline Sgr.A* j & $17^{\mathrm{h}} 46^{\mathrm{m}}$ & $-29^{\circ} 00^{\mathrm{m}}$ & $1.6 \times 10^{8}$ & $05^{\mathrm{h}} 46^{\mathrm{m}}$ & $+29^{\circ} 00^{\mathrm{m}}$ & \\
\hline
\end{tabular}

a Data for planets from https://ssd.jpl.nasa.gov/horizons.cgi . Coordinate system is Sun centered, equatorial.

b Dates for aphelion and perihelion from www.skycaramba.com/aphelions.shtml and www.skycaramba.com/perihelions.shtml .

c Distance from the center of the Sun.

d Dates: 7 Feb. 2017, 23 Mar. 2017.

e Dates: 12 Jun. 2017, 20 Feb. 2017.

f Dates: 3 Jul. 2017, 4 Jan. 2017.

g Dates: 07 Oct. 2017, 29 Oct. 2016.

h Dates: 14 Jul. 2076.

i Dates: 24 Oct. 2005.

j J2000 Wikipedia. 


\section{REFERENCES}

\section{References}

Bartlett, R., 2020, NON-COMPUTABILITY AND UNPREDICTABILITY ARE SO YESTERSDAY: WITH COMPUTABLE AND PREDICTABLE COSMIC STRUCTURE, PLUS IMPLICATIONS FOR MATHEMATICS AND SCIENCE, FQXi 2020 contest, https://fqxi.org/community/forum/topic/3382 .

Hodge, J.C., 2006. Scalar potential model of the Pioneer Anomaly, preprint http://arxiv.org/abs/astro-ph?0612567v1.pdf .

Hodge, J.C., 2019, STOE explains "Planet 9" , IntellectualArchive, Vol.8, No. 2, P.13, http://intellectualarchive.com/?link=item\&id=2089. 


\title{
Is Gravity the Only Way to Bend Space-Time? \\ Mark Zilberman, M.Sc. \\ Shiny World Corp. (Canada)
}

\begin{abstract}
The purpose of this article is to raise the question, "Is gravity the only way to bend the space-time, or can there be other ways to bend space-time?" The answer to this question can be either, A) a description of the mechanism of non-gravitational curvature of space-time, or B) proof that gravity is the only method to bend space-time and there is no other way. Since modern science can prove neither A nor B, the question raised in this article remains open.

This article also discusses and rejects the objection that non-gravitational curvature of space-time would be observed as a kind of "additional" gravity, what is not taking place. The scale and strength of non-gravitational curvature is important. Also, the non-gravitational curvature of space-time does not necessarily have the U-shaped form, which we observe as a gravity.
\end{abstract}

The explanation of gravity by the curvature of space-time generated by the presence of massenergy was put forward by Einstein in his works Die Feldgleichungen der Gravitation [1] and Die Grundlage der allgemeinen Relativitätstheorie [2], published in 1915 and 1916 respectively. The Theory of General Relativity has been brilliantly confirmed in many fields of physics and is used in astronomy, engineering, astronautics, atomic physics, etc.

Thus, General Relativity and subsequent experiments:

A. created a precedent that space-time can be bent;

B. proposed the presence of mass-energy as the mechanism of this curvature;

C. confirmed this model in many experiments in various fields of science and technology.

Thus, a precedent has been created. It is possible to bend space-time and there is at least one mechanism that can do this. However (as far as the author knows) no one has ever proved that gravity is the only way to bend space-time.

Therefore, may there be some other mechanism of space-time curvature?

This question is unusual, but in the absence of evidence that gravity is the only way to bend space-time, it is a legitimate one.

The author's goal, of course, is not to propose another way to bend space time. Perhaps it simply does not exist. This article is merely a statement of the problem, which in all probability has only two solutions:

A. a description of the mechanism of non-gravitational curvature of space-time, or

B. proof that gravity is the only method to bend space-time and there is no other way. 
Since modern science can prove neither A nor B, the question raised in this article remains open.

\section{Discussion}

The question posed in the article may cause a natural objection. Since the curvature of spacetime is perceived by us as gravity, then any non-gravitational curvature of space-time will also be perceived by us as some kind of "additional" gravity. And since we do not observe anything like this in nature, only the gravitational curvature of space-time is possible.

This objection, however, is untenable for three reasons.

First, the scale at which the non-gravitational curvature of space-time occurs is important. If it takes place on a scale of, say, several meters, then we probably will not notice any effect on the Earth's motion. If it occurs in space on a planetary scale, then when observing the stars, we also will not notice it, etc.

Secondly, intensity is also important. Gravitational curvature of space-time depends on the mass of the object. The larger the mass, the stronger (and more noticeable) the curvature of space (the stronger is the gravity of the object). For a small mass, the gravitational curvature of space is negligible. The same thing with non-gravitational curvature of space-time. It can be so weak that it is not detected as a deviation of gravity.

Thirdly, most important. We perceive gravity as the U-shaped curvature of space-time. (Figuratively speaking, for a black hole it is more like a Y-shaped curvature of space-time.) However, we do not know how the curvature of space-time of a different form is perceived. Therefore, speaking of the non-gravitational curvature of space-time, we cannot postulate that it will also be U-shaped, and that it will be perceived by us as some kind of additional gravity.

Once again, the purpose of the article is not to prove that a non-gravitational curvature of spacetime is possible, but only to pose the question about it.

\section{References}

1. Albert Einstein. Die Feldgleichungen der Gravitation // Sitzungsberichte der Preussischen Akademie der Wissenschaften zu Berlin. — 1915. — 25 November. — P. 844 - 847.

2. Albert Einstein. Die Grundlage der allgemeinen Relativitätstheorie // Annalen der Physik. 1916. — V. 354, \# 7. - P. 769-822. 


\title{
Simplified Method of Visualization of Light Distribution at the Initial Stage of Design of Symmetrical LED Luminaires
}

\author{
Lidiya Koval \\ Associate Professor, PhD in Art History, \\ Doctorate in the Department of Architectural Structures \\ Kyiv National University of Construction and Architecture, Ukraine
}

\begin{abstract}
The article offers a simplified method of visualization of light distribution of LED luminaires, with due consideration of specifics of LED as illuminant: namely, its dime size that allows to consider it a point source, and broad installation opportunities - combination with different shapes and surfaces. The efficacy of the proposed method is confirmed by calculation and visualization of light distribution of symmetrical LED luminaires. Among them there are three rotationally symmetrical luminaires that demonstrate which way the shape of shade influences luminous distribution. Main results of the research have been obtained owing to the elaboration and use demonstration of a simplified methodology of calculation and visualization of light distribution of symmetrical LED luminaires grounding on the combination of graphical-analytical method and computing methods with the use of free software, namely IESGen, LDT Editor, IESviewer.
\end{abstract}

Keywords: symmetrical LED luminaires, visualization of light distribution, initial stage of design.

Problem formulation. The most widely spread record format of photometric quantities related to light distribution of luminaires is IES format which has become standard in some countries [1]. It is accepted by almost all the software featuring 3D-modelling, and its main convenience is that it has the form of a text that is up to editing and correcting. However, this format secures allocation of all the necessary photometric quantities of a luminaire, without providing any graphic image of the present light distribution. Thus, further construction of diagrams and visualization of light distribution of a luminaire requires the use of additional software products.

Analysis of recent research and publications. The vast majority of special computer programs elaborated for designing optical and luminous systems are commercial. Some global producers of LED products create special free software [2], that assist designers in choosing the most relevant LEDs for the designed illumination device by means of matching by several set-up parameters, i.e. directed luminous flux and specific value of efficiency rate of secondary optics and power source. Such applications are an important informative supplement for general design of a luminaire but are not related to prediction and visualization of its luminous intensity distribution. Thus, aiming at passing all the stages of 
visualization of luminous intensity distribution by means of free software and at creating a file, which is applicable for further calculations of illuminance of certain objects, a combination of several program applications has been employed in the process of elaboration, namely IESGen [3], LDT Editor [4], IESviewer [5].

The purpose of the article. So, the aim of the present paper is the creation and demonstration of the use of simplified method of visualization of light Distribution in symmetrical LED luminaires, employing computer methods based on the combination of software tools from several free applications.

Results. The procedure of calculation and isocandela curves construction included the following steps. Initially, calculations were performed according to the data of LED illumination and basing on structural layout of luminaires. Afterwards, an IES file was created by means of IESGen software, and later, in the program LDT Editor by DIAL GmbH all calculations on every luminaire were input and corresponding diagram of luminous intensity distribution were obtained. While saving the corrected file, LDT Editor converts it into its own format with .ldt extension. However, this does not make a problem since this format was created for editing luminaire files using free programs for calculation of lighting designed by DIAL GbmH, and it easily integrates into them, allowing both to perform preliminary rough calculations of lighting by means of DIALux Light program, and to create spatial visualizations of luminous intensity distribution of a luminaire as well as to simulate conditional borders of its photometric body in full version of DIALux program. Besides, .ldt format is friendly for IESviewer program which enabled to create graphic visualizations of preliminary constructed diagram of luminous intensity distribution not schematically - in the form of diagrams, but realistically - as a photo of luminous intensity distribution of a luminaire.

Taking into account that a LED can be considered a point source of light with definite luminous intensity distribution [6, p. 47-77], it is worth mentioning that the majority of modern frameless powerful LEDs (without primary and secondary optics) have type D diagram, i.e. cosine one (according to the classification which is wide-spread in Ukraine), or $\mathrm{BZ}_{5}$ (according to British zonal classification), or circular (according to the typology, singled out on the basis of these catalogues) [7]. Considering that luminous intensity distribution with such a curve is believed to be appropriate for general lighting of accommodations and public 
spaces, the design of this method was elaborated with the use of LED luminaires made of frameless LEDs.

The elaborated method is based on the hypothesis of unified value of luminous intensity of a separate LED $I=1 \mathrm{~cd}$ in calculations for illumination devices containing LEDs with the same luminous intensity distribution, and of unified value of a separate LED $\Phi=1$ $1 \mathrm{~m}$ in calculations for complicated illumination devices which contain LEDs with different luminous intensity distribution.

We assume that a LED is a point source. Assuming for the sake of calculation convenience that luminous intensity value of LED $I=1 \mathrm{~cd}$, distance from the source to reference surface $r=1 \mathrm{~m}$ with the spacing between reference points equal to $1^{\circ}$ (symmetry of luminous distribution allows to limit values for angle of arrival of a beam from $0{ }^{\circ}$ to $900^{\circ}$ ), we can define illuminance $E(\mathrm{~lx})$ using the formula [8, p. 141-144]:

$$
E=\frac{I \cos \theta}{r^{2}}
$$

in which $E$ - illuminance in reference point; $\theta$ - angle of arrival of a beam (an angle between a falling beam and a a perpendicular line put against the surface in the point of the beam arrival); $r$ - the distance between the source to the surface (the reference point on the surface).

In order to demonstrate the influence of the shape of a shade on luminous intensity distribution, we calculated diagram of luminous intensity distribution for three rotationally symmetrical luminaires with radial symmetry of LED allocation on the mounting surface. Calculations were performed according to formula (1), with the pitch between calculated points of 1 ; balance of luminous intensity distribution allowed to limit the range of values for angle of beam arrival $0^{\circ}$ to $90^{\circ}$. The results of the research that represent constructive schemes of luminaires and visualizations of luminous intensity distribution is given in Table 3.

During calculation the following aspects were taken into account:

- Similar frameless LEDs with the same luminous intensity distribution were used as sources, so $I=1 \mathrm{~cd}$ is admitted as calculated value of luminous intensity for each separate LED. 
- In other equal similar conditions illuminance will be proportional to luminous intensity of the source, and two similar sources, allocated nearby, will send in the given direction twice more luminous energy than one source, and such sources can be replaced by one, with bigger luminous intensity. That is why maximum calculated luminous intensity of a luminaire $I_{p l}$ will equate in the calculated plane to the product of luminous intensity of one LED $I$ and the total number of LEDs $n$ in a luminaire. Afterwards it is necessary to determine, which part of the maximum luminous intensity is provided by each LED for calculated plane, depending on the quantity of LEDs that are intersected by a definite calculated plane.

- For performing calculations in case of considerable distances from calculated point of illuminated surface to luminaire, comparing to the size of the luminaire itself, the distance from each separate LED can be considered equal to the average for all the LEDs distance from the provisional central point of the luminaire to calculated point. In this case illuminance of any point on calculated surface will be proportional to that quantity of LEDs that is not cut off by luminaire walls.

- As a provisional central point of a luminaire, we chose the point which lies at the intersection of vertical symmetry axis, which passes through geometrical center of the luminaire, and horizontal axis, which passes through geometrical center of the upwardmost LED (LEDs). 
Table 3. Constructive schemes and visualization of luminous intensity distribution of rotationally symmetrical LED luminaires with shades of different shape
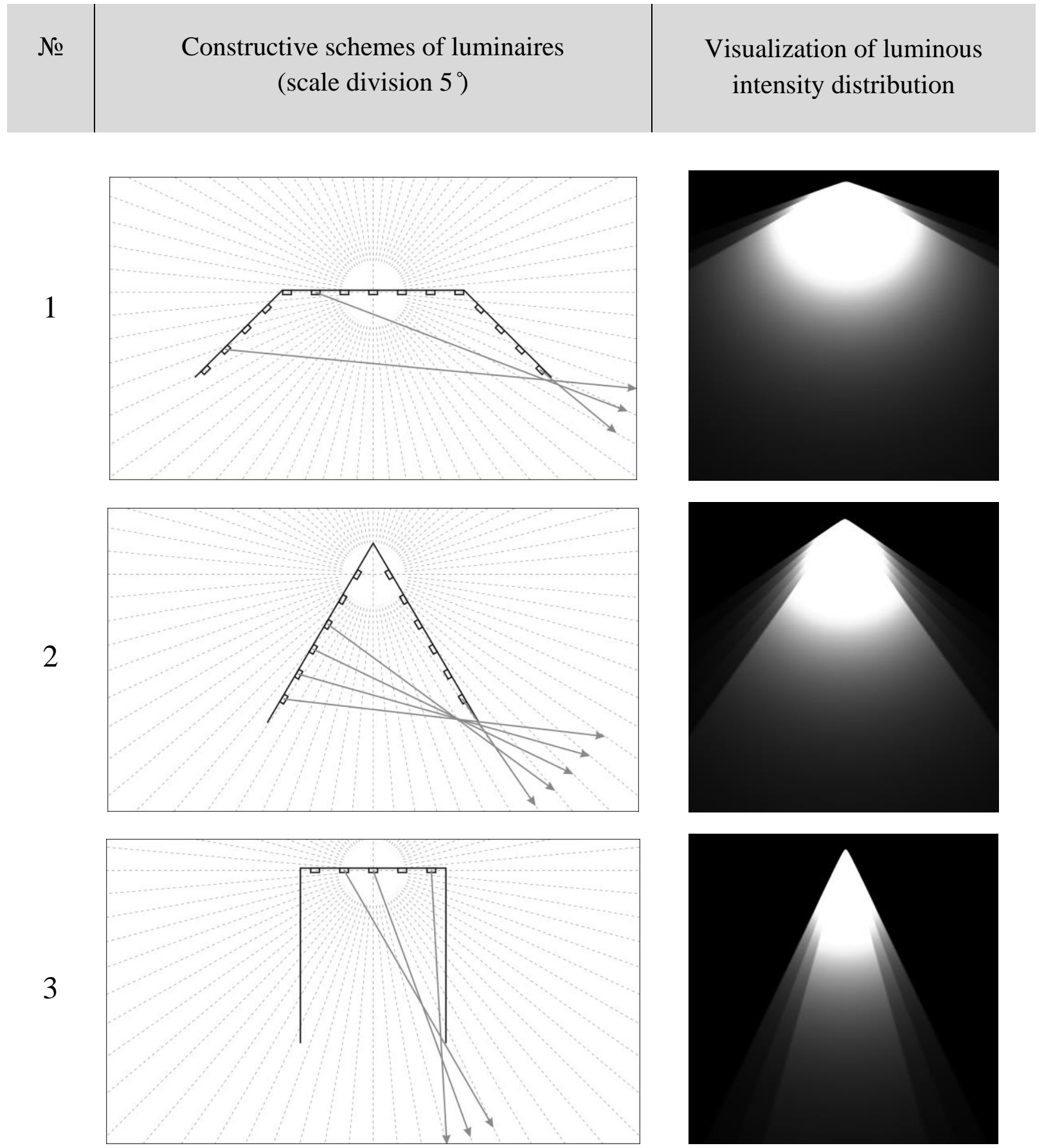

Then we will analyze the process of construction visualization of light distribution given in Table 3.

Luminaire № 1 in Table 3, as it is obvious from constructive scheme, contains only 15 LEDs that intersect with calculated plane, they are not cut off to calculated angle $60^{\circ}$, from 
$61^{\circ}$ to $65^{\circ} 6$ LEDs are not cut off, from $66^{\circ}$ to $70^{\circ}-2$ LEDs are not cut off, and light does not come to calculated angles starting with $71^{\circ}$.

Luminaire № 2 inTable 3, as it is obvious from its constructive scheme, has only 12 LEDs that are intersected by calculated plane, they are not cut off to calculated angle $35^{\circ}$, from $36^{\circ}$ to $40^{\circ} 4$ LEDs are not cut off, from $41^{\circ}$ to $45^{\circ}-3$ LEDs are not cut off, from $46^{\circ}$ to $50^{\circ}-2$ LEDs are not cut off, from $51^{\circ}$ to $55^{\circ}-1$ LED is not cut off and light does not come to calculated angles starting with $56^{\circ}$.

Luminaire № 3 in Table 3, as it is obvious from its constructive scheme, has only 5 LEDs that are intersected by calculated plane, they are not cut off to calculated angle $15^{\circ}$, from $16^{\circ}$ to $20^{\circ} 3$ LEDs are not cut off, from $21^{\circ}$ to $25^{\circ}-2$ LEDs are not cut off, and light does not come to calculated angles starting with $26^{\circ}$.

Conclusions. Suggested method of visualization of light distribution of symmetrical LED luminaires does not take into account either light diffusing by the inner surface of luminaire shade (or LED mounting surface), or absorptance and reflectivity of the material which this surface is made of, etc., but this method provides information on the influence of the shape of a designed luminaire shade on luminous intensity distribution, and the visualization of light distribution can become the basis for their further refinement and perfection at later stages of design.

In further studies in this research direction it is reasonable to focus attention on peculiarities of calculation and construction of luminous intensity distribution of LED luminaires in cases when distance from calculated point to a luminaire is insignificant comparing to its size.

\section{References}

1. Illuminating Engineering Society of North America. Standard file format for electronic transfer of photometric data. Report LM-63-95. New York: IESNA, 1995.

2. CREE. Product characterization tool. Retrieved 18 November 2019 from http://pct.cree.com/dt/index.html

3. Karba. IESGen Version 4.0., 2006. LightOnline. Retrieved 18 November 2019 from http://www.lightonline.ru/documents/Other/IES_Generator.html

4. DIAL GmbH. LDT Editor. Free tool for ldt files. Retrieved 18 November 2019 from https://www.dial.de/en/dialux/ldt-editor/ 
5. Legotin A. IESviewer (photometric viewer), 2001. Retrieved 18 November 2019 from http://photometricviewer.com/download.html

6. Koval L. Design and LED technology. Zaporizhzhia National Technical University, Zaporizhzhia: ZNTU, 2014.

7. Filip A, Maier V, Pavel SGh, Polec V. Polar curves for indoor direct lighting luminaires and BZ classification. Acta Electrotehnica, Mediamira Science Publisher 2012; 53(2): $167-174$.

8. Miakishev G., Bukhovtsev B. Physics. Kyiv: Radianska shkola, 1974.

\section{Translation of the Author's Name and Affiliation to the Original Language}

Коваль Лідія Михайлівна, кандидат мистецтвознавства, доцент, докторант кафедри архітектурних конструкцій, Київський національний університет будівництва i архітектури, Україна 


\title{
THE PRINCIPLES OF THE CONCEPTUAL ORGANIZATION AND CONSTRUCTION OF PATENT PROTECTION
}

\section{for smart integrative technical systems containing complex subsystems, managed and on-line controlled at the level of capabilities of quantum computers containing elements of artificial intelligence and artificial neural networks}

\author{
Yerzhan Kusparmakov \\ - Engineer, Inventor, Doctor of Philosophy (PhD) Major in Computer Science \\ - Professional Member of the New York Academy of Science \\ - Academician of the International Informatization Academy \\ - Chief Product Manager at SAMSUNG Electronics \\ e-mail: kusparmakov@gmail.com
}

\begin{abstract}
The integration of software solutions and mobile applications into innovative technical systems of all levels in the current process of transforming classical technologies into smart technologies and transforming the entire hierarchy of technical systems and their interconnections into smart technical systems is the basis for the formation of such innovative products that are most in demand on the markets.

As the practice of such innovative development shows, the use of methods and techniques of the inventive process adapted to today's elemental base and modern composite materials from generating an idea to the complete formation of materials for an application for an invention can be successfully carried out with the well-known Theory and Algorithm for solving inventive problems.

Of particular importance in the innovation process are relations with investors and potential strategic partners in the commercial application of the created innovative product.

Keywords: Patent, Licensing, Quantum Computers, Artificial Intelligence (AI), Artificial Neural Networks (ANN), Augmented Reality (AR), Smart technology
\end{abstract}

The integration of software solutions and mobile applications into innovative technical systems of all levels in the current process of transforming classical technologies into smart technologies and transforming the entire hierarchy of technical systems and their interconnections into smart technical systems is the basis for the formation of such innovative products that are most in demand on the markets.

As the practice of such innovative development shows, the use of methods and techniques of the inventive process adapted to today's elemental base and modern composite materials from generating an idea to the complete formation of materials for an application for an invention can be successfully carried out with the well-known Theory and Algorithm for solving inventive problems. 
The use of Theory of Inventive Problem Solving (TIPS) and Algorithms for Solving Inventive Problems (ASIP) is of course only one of many possible analytical tools in the preparation of a patent application.

The relations with investors and potential strategic partners in the commercial application of the created innovative product are of particular importance in the innovation process.

To convince the investor that investments in the proposed project will bring financial success, it is necessary to explain in detail the essence of the project and to reveal all technological and innovative secrets to the experts.

It is impossible without serious protection of the engineering solutions that form the basis of the innovative product at all stages of the project development.

Non-traditional types and forms of collective investment have now appeared and are successfully developing, and this also motivates the project authors to constantly search for more and more reliable forms of protection of their inventions.

Provisional Patent Application is an inexpensive and reliable protection option for up to 1 year.

The use of TIPS and ASIP, as already mentioned above, with the necessary and sufficient updating of the basic techniques and methods, is only one of the many possible tools in the preparation of the patent application and in many cases requires system modification and optimization taking into account new innovative circumstances.

For each specific case, taking into account all circumstances, reasons, objectives and conditions, and taking into account the results of patent forecasting, in full compliance with the current US Patent Law (America Invents Act), an applicant company or an individual inventor may have, and most often need, an integrative program in which the original system and structure of the patent application are precisely developed.

A patent licensing strategy, including a consistent patent forecasting process, can also be, and most often should be, developed for each individual applicant or inventor.

This manual is also fully suitable for compiling application materials for filing (Non Provisional Patent Application ..., - Utility Patent Application).

\section{APPARATUS AND METHOD FOR ...}

The title of the proposed invention should contain not only a brief definition of the nature of the engineering solution, but also an extremely brief commercial description of its impact on the market within the scope of the proposed engineering solution. The title should not contain any advertising characteristics..., - such as effective, best, etc. 
In the title of the invention, it is very important to take into account the market conditions existing at the time of application filing. If the purpose of the authors (or their companies and financial partners) and applicants is to further attract additional investment in the development of the project, the basis of which should be the claimed engineering solution, it is preferable to consider in the title the known technological and commercial interests of potential partners.

Since the proof of non-obviousness of the claimed engineering solution is very important for a patent expert in the United States, it is extremely important to take into account the criteria and characterization of non-obviousness in the title of the invention.

Technical systems related to the so-called smart technologies and equipment, as a rule, should include the software part, which in itself is not a technical solution and cannot be classified as a technical system.

But since software solutions are the basis of the control and monitoring systems of any modern smart technology, this should be taken into account in the name of the invention, that is, the name of the invention should be presented in the form: - Device, program, system and method; or - An apparatus, program, system and method.

In addition, when classifying a technical system as a smart system having elements of artificial intelligence and artificial neural networks, it is also necessary to have information storages with the corresponding amount of information and corresponding speed.

This factor should be reflected in the name, for example ..., - The device, program, system with memory drives, and the associated method of use.

\section{REFERENCE TO RELATED APPLICATIONS}

[0001] This application claims the priority benefit under 35 U.S.C. $\$ 119($ e) of U.S. Provisional Application Nos. 61/, filed...; and 61/, filed... The entire disclosures of each of these applications are incorporated herein by reference..., - this section contains information on all provisional patent applications that have been filed by the authors of the invention or the applicant prior to the execution and filing of this application.

If the provisional patent applications in the United States (Provisional Patent Applications) have not been filed, this section of the application should specify information on all previous applications on this topic and this technical field, which were filed by the authors of the invention or by the applicant (in the name of the authors of this application or other authors) before the execution and filing of this application.

\section{TECHNICAL FIELD}


[0002] The invention relates to apparatus and methods for..., - this section describes the field of methods and technology to which the claimed engineering solution relates.

The nature of belonging to one or another technical field should be clearly reasoned and related to the distinctive features of the claimed engineering solution by cause-effect relations characterized and formulated understandable for a semi-skilled specialist in this field.

If the distinctive features of the claimed engineering solution are obvious for one field and not obvious for another, it is necessary to stop at this and try to give an analytical explanation of this phenomenon.

It is especially important to characterize the problems and contradictions peculiar to and obvious for the technical field selected for the invention and for the development of this thesis for the field of methods and technology to which the claimed engineering solution relates.

Due to the fact that, unlike the European Patent Office, in the United States, under certain conditions, a patent can be granted for a computer program, it is preferable, if possible, to have an integration solution for the claimed engineering solution in the form of..., - Program, system, method and device for their application.

\section{DESCRIPTION OF THE RELATED TECHNOLOGY}

[0003] - this section consists of two parts. The first part provides a more specified, definite, local analytical assessment of the field of methods and technology, to which the claimed engineering solution relates. The most characteristic features of the technology, its known disadvantages and advantages are described.

[0004] - in this part of the section, it is preferable to start the search and preliminary analysis of the contradictions of a general nature, which are known and characteristic of the technology to which the inventors relate it.

\section{BACKGROUND OF THE INVENTION}

[0005] - this section, which is one of the most important in the application, provides an indepth analysis of patent search results for all options and sources of published patent information, at least for the US Patent Office, and better if for the European Patent Office, and even better if you give a comparative analysis of known engineering solutions and, in conclusion, bring the results of structural element-wise analysis. 
After that, using the TIPS and ASIP tools, it is necessary to search, formulate and comparatively analyze all kinds of contradictions in the known engineering solutions and determine and characterize a possible ideal final result.

Then it is necessary to analyze the possibility of the fundamental achievement of the ideal final result in the claimed engineering solution by using equipment, technology, materials and means of control and management, known at the time of application filing.

\section{SUMMARY}

[0006] According to some embodiments of the invention..., - this section defines the first local ultimate objective of the invention and defines the way to achieve it within the framework of compliance with the claims; provides identification of the local objective and determines whether it is enough to achieve the objective in view in order to state the achievement of at least a partial or local ideal final result.

[0007] In some embodiments, programs, systems and methods of..., - this section defines the second local ultimate objective of the invention and defines the way to achieve it within the framework of compliance with the claims; provides identification of the local objective and determines whether it is enough to achieve the objective in view in order to state the achievement of at least a partial or local ideal final result.

[0008] In some embodiments, further programs, systems and methods for..., - this section defines the third local ultimate objective of the invention and defines the way to achieve it within the framework of compliance with the claims; provides identification of the local objective and determines whether it is enough to achieve the objective in view in order to state the achievement of at least a partial or local ideal final result.

[0009] Also in some further embodiments, programs, systems and methods of..., - this section defines the first integrated local ultimate objective of the invention and defines the way to achieve it within the framework of compliance with the claims; provides identification of the local objective and determines whether it is enough to achieve the objective in view in order to state the achievement of at least a partial or local ideal final result.

[0010] In some embodiments, an apparatus is provided with..., - this section defines the first local ultimate objective of the invention related cause-effect with the second local ultimate objective of the invention and defines the way to achieve the specified objective integration within the framework of compliance with the claims; provides identification of the local integrated objective and determines whether it is enough to achieve this objective in view in order to state the achievement of at least a partial or local ideal final result. 
[0011] - this section defines the first local ultimate objective of the invention related causeeffect with the second and third local ultimate objectives of the invention and defines the way to achieve this objective integration within the framework of compliance with the claims; provides identification of the local integrated objective and determines whether it is enough to achieve this objective in view in order to state the achievement of at least a partial or local ideal final result.

[0012] In some embodiments, an apparatus for..., - this section defines the first local ultimate objective of the invention in terms of solutions for the apparatus related cause-effect with the second local ultimate objective of the invention also related to the apparatus and defines the way to achieve the specified objective integration within the framework of compliance with the claims; provides identification of the local integrated objective and determines whether it is enough to achieve this objective in view in order to state the achievement of at least a partial or local ideal final result.

[0013] In some other embodiments, an apparatus is provided with a system for..., - in this section, one of the known systems, that is necessary for its declared duty cycle, is added to the apparatus and the presence or absence of a jump effect is analyzed.

[0014] In some other embodiments, an apparatus is provided with a system for..., - in this section, the second of the known systems, that is necessary for its declared duty cycle, is added to the apparatus and the presence or absence of a jump effect is analyzed.

[0015] In some embodiments of the various apparatus, the..., - in this section, a combination of the known systems integrated with the apparatus into an integrative system, that is necessary for its declared duty cycle, is added to the apparatus and the presence or absence of a jump effect is analyzed.

[0016] - in this section, a combination of the known systems, that is necessary for its declared duty cycle, is added to the apparatus one by one, and the presence or absence of a jump effect is analyzed.

[0017] The system for..., - in this section, a structural analysis of the system (s) of the engineering solution is carried out and the sufficiency of the presence of the combination of all necessary systems in the engineering solution to ensure the achievement of an ideal final result is monitored.

[0018] Structurally, the apparatus can be provided with a...,- in this section, a structural analysis of the nodes and components of the apparatus is carried out.

[0019] In the apparatus, the system for..., - this section shows how design features affect the achievement of an ideal final result. 
[0020] The apparatus can also comprise a system for..., - this section considers the possibility of including various additional functionally independent and autonomous systems into the apparatus that can enhance the effect of the use of the proposed invention and thereby ensure a more confident achievement of the ideal final result.

[0021] In some embodiments, the method of operation of the device involves..., - this section analyzes local and integral objectives in dynamics or action, analyzes methods of achieving the objectives, and compares parameters after achieving a specified objective with an ideal final result.

[0022] An external..., - this section considers additional possible aspects of improving and modifying objectives and ways to achieve them, as well as analyzes and assesses the potential for achieving additional components of an ideal final result.

[0023] The following can be provided to..., - in this section, various actions and constructive improvements are considered that can complement the essence of the ideal final result with new content.

[0024] In some embodiments..., - this section gives the final definition of the fully integrated local ultimate objective of the invention and defines the way to achieve it within the framework of compliance with the claims.

The identification of the local and fully integrated objective in view is given and it is determined whether it is enough to achieve this objective in order to state the achievement of at least a partial or local ideal final result.

\section{BRIEF DESCRIPTION OF THE DRAWINGS}

[0025] The invention will be better understood from the Detailed Description and from the appended drawings, which are meant to illustrate and not to limit the invention. The Figures are not necessarily drawn to scale, nor are the relative sizes of parts within the Figures necessary in proportion to one another, - the invention will be better understood from the detailed description and from the appended drawings, which are meant to illustrate and not to limit the invention. The Figures are not necessarily drawn to scale, nor are the relative sizes of parts within the Figures necessary in proportion to one another.

[0026] Figure 1 is an example of a..., - this figure represents a general schematic flowchart of an engineering system/supersystem, in which all subsystems and their communication with each other as well as outputs and lines of communication with the supersystem are displayed with a focus on the functional features and differences integrated into the supersystem of software elements in combination with the management and control systems of the supersystem comprising elements of artificial intelligence and artificial neural networks. 
[0027] Figure 2 is an example of a ...,- this figure represents a general schematic flowchart of a leading and central engineering system/subsystem, in which all functionally related subsystems and their communication with each other as well as outputs and lines of communication with the supersystem are displayed with a focus on the functional features and differences integrated into the supersystem and, if possible, subsystems of software elements, in combination with the management and control systems of the supersystem comprising elements of artificial intelligence and artificial neural networks.

Technical details and software functions, as well as algorithms characterizing the nonobviousness of these solutions in combination with the general principles of use of elements of artificial intelligence and artificial neural networks are also reflected.

[0028] Figure 3 shows an example..., - this figure represents a general schematic flowchart of an auxiliary or local first engineering system/subsystem, in which all functionally related subsystems and their communication with each other as well as outputs and lines of communication with the supersystem are displayed with a focus on the functional features and differences integrated into the supersystem and, if possible, subsystems of software elements, in combination with the management and control systems of the supersystem comprising elements of artificial intelligence and artificial neural networks.

Technical details and software functions, as well as algorithms characterizing the nonobviousness of these solutions in combination with the general principles of use of elements of artificial intelligence and artificial neural networks are also reflected.

[0029] Figure 4 shows an example of components of the..., - this figure represents a general schematic flowchart of an auxiliary or local second engineering system/subsystem, in which all functionally related subsystems and their communication with each other as well as outputs and lines of communication with the supersystem are displayed with a focus on the functional features and differences integrated into the supersystem and, if possible, subsystems of software elements, in combination with the management and control systems of the supersystem comprising elements of artificial intelligence and artificial neural networks.

Technical details and software functions, as well as algorithms characterizing the nonobviousness of these solutions in combination with the general principles of use of elements of artificial intelligence and artificial neural networks are also reflected.

[0030] Figure 5 shows an example of a..., - this figure represents a general schematic flowchart of an auxiliary or local third engineering system/subsystem, in which all functionally related subsystems and their communication with each other as well as outputs and lines of communication with the supersystem are displayed with a focus on the functional features and differences integrated into the supersystem and, if possible, subsystems of software elements, in combination with the management and control systems of the supersystem comprising elements of artificial intelligence and artificial neural networks. 
Technical details and software functions, as well as algorithms characterizing the nonobviousness of these solutions in combination with the general principles of use of elements of artificial intelligence and artificial neural networks are also reflected.

[0031] Figure 6 is a..., - the illustrations to Figure 1 made as dependent graphic structures to dependent claims relating to independent claim 1.

[0032] Figure 7 shows an example of a..., - the illustrations to Figure 2 made as dependent graphic structures to dependent claims relating to independent claim 2.

[0033] Figure 8 is an example of a..., - the illustrations to Figure 3 made as dependent graphic structures to dependent claims relating to independent claim 3.

[0034] Figure 9 is an example of a...,- the illustrations to Figure 4 made as dependent graphic structures to dependent claims relating to independent claim 4.

[0035] Figure 10 is an example of a..., - the illustrations to Figure 5 made as dependent graphic structures to dependent claims relating to independent claim 5.

[0036] Figure 11 is an example of a...,- this figure represents a general schematic flowchart or interaction algorithm of an auxiliary or local first engineering system/subsystem, in which all functionally related subsystems and their communication with each other as well as outputs and lines of communication with the supersystem are displayed with a focus on the functional features and differences integrated into the supersystem and, if possible, subsystems of software elements, in combination with the management and control systems of the supersystem comprising elements of artificial intelligence and artificial neural networks.

Technical details and software functions, as well as algorithms characterizing the nonobviousness of these solutions in combination with the general principles of use of elements of artificial intelligence and artificial neural networks are also reflected.

[0037] Figure 12 is an example of an..., - this figure represents a general schematic flowchart or interaction algorithm of an auxiliary or local second, in order of importance, engineering system/subsystem, in which all functionally related subsystems and their communication with each other as well as outputs and lines of communication with the supersystem are displayed with a focus on the functional features and differences integrated into the supersystem and, if possible, subsystems of software elements, in combination with the management and control systems of the supersystem comprising elements of artificial intelligence and artificial neural networks.

Technical details and software functions, as well as algorithms characterizing the nonobviousness of these solutions in combination with the general principles of use of elements of artificial intelligence and artificial neural networks are also reflected. 
[0038] Figure 13 is an example of a..., - this figure represents a general schematic flowchart or interaction algorithm of an auxiliary or local third, in order of importance, engineering system/subsystem, in which all functionally related subsystems and their communication with each other as well as outputs and lines of communication with the supersystem are displayed with a focus on the functional features and differences integrated into the supersystem and, if possible, subsystems of software elements, in combination with the management and control systems of the supersystem comprising elements of artificial intelligence and artificial neural networks.

Technical details and software functions, as well as algorithms characterizing the nonobviousness of these solutions in combination with the general principles of use of elements of artificial intelligence and artificial neural networks are also reflected.

[0039] Figure 14 is an example of a..., - this figure represents a general schematic flowchart or interaction algorithm of an auxiliary or local fourth, in order of importance, engineering system/subsystem, in which all functionally related subsystems and their communication with each other as well as outputs and lines of communication with the supersystem are displayed with a focus on the functional features and differences integrated into the supersystem and, if possible, subsystems of software elements, in combination with the management and control systems of the supersystem comprising elements of artificial intelligence and artificial neural networks.

Technical details and software functions, as well as algorithms characterizing the nonobviousness of these solutions in combination with the general principles of use of elements of artificial intelligence and artificial neural networks are also reflected.

[0040] Figure 15 is an example of a..., - this figure represents a general schematic flowchart or interaction algorithm of an auxiliary or local fifth, in order of importance, engineering system/subsystem, in which all functionally related subsystems and their communication with each other as well as outputs and lines of communication with the supersystem are displayed with a focus on the functional features and differences integrated into the supersystem and, if possible, subsystems of software elements, in combination with the management and control systems of the supersystem comprising elements of artificial intelligence and artificial neural networks.

Technical details and software functions, as well as algorithms characterizing the nonobviousness of these solutions in combination with the general principles of use of elements of artificial intelligence and artificial neural networks are also reflected.

[0041] Figure 16 is an example of a..., - the illustrations to Figure 11 made as dependent graphic structures to dependent claims relating to independent claim 11. 
[0042] Figure 17 shows an example of..., - the illustrations to Figure 12 made as dependent graphic structures to dependent claims relating to independent claim 12.

[0043] Figure 18 is an example of a..., - the illustrations to Figure 13 made as dependent graphic structures to dependent claims relating to independent claim 13.

[0044] Figure 19 is an example of a....., - the illustrations to Figure 14 made as dependent graphic structures to dependent claims relating to independent claim 14.

[0045] Figure 20 is an example of a..., - the illustrations to Figure 15 made as dependent graphic structures to dependent claims relating to independent claim 15.

[0046] Figure 21 is an example of a..., - this figure represents a general schematic flowchart or algorithm of complex integration of an auxiliary or local first, in order of importance, engineering system/subsystem into a central supersystem, and all functionally related subsystems and their communication with each other as well as outputs and lines of communication with the supersystem are displayed in such engineering system/subsystem, with a focus on the functional features and differences integrated into the supersystem and, if possible, subsystems of software elements, in combination with the management and control systems of the supersystem comprising elements of artificial intelligence and artificial neural networks.

Technical details and software functions, as well as algorithms characterizing the nonobviousness of these solutions in combination with the general principles of use of elements of artificial intelligence and artificial neural networks are also reflected.

[0047] Figure 22 is an example of a..., - this figure represents a general schematic flowchart or algorithm of complex integration of an auxiliary or local second, in order of importance, engineering system/subsystem into a central supersystem, and all functionally related subsystems and their communication with each other as well as outputs and lines of communication with the supersystem are displayed in such engineering system/subsystem, with a focus on the functional features and differences integrated into the supersystem and, if possible, subsystems of software elements, in combination with the management and control systems of the supersystem comprising elements of artificial intelligence and artificial neural networks.

Technical details and software functions, as well as algorithms characterizing the nonobviousness of these solutions in combination with the general principles of use of elements of artificial intelligence and artificial neural networks are also reflected.

[0048] Figure 23 is an example of a..., - this figure represents a general schematic flowchart or algorithm of complex integration of an auxiliary or local third, in order of importance, engineering system/subsystem into a central supersystem, and all functionally related 
subsystems and their communication with each other as well as outputs and lines of communication with the supersystem are displayed in such engineering system/subsystem, with a focus on the functional features and differences integrated into the supersystem and, if possible, subsystems of software elements, in combination with the management and control systems of the supersystem comprising elements of artificial intelligence and artificial neural networks.

Technical details and software functions, as well as algorithms characterizing the nonobviousness of these solutions in combination with the general principles of use of elements of artificial intelligence and artificial neural networks are also reflected.

[0049] Figure 24 is an example of a..., - this figure represents a general schematic flowchart or algorithm of complex integration of an auxiliary or local fourth, in order of importance, engineering system/subsystem into a central supersystem, and all functionally related subsystems and their communication with each other as well as outputs and lines of communication with the supersystem are displayed in such engineering system/subsystem, with a focus on the functional features and differences integrated into the supersystem and, if possible, subsystems of software elements, in combination with the management and control systems of the supersystem comprising elements of artificial intelligence and artificial neural networks.

Technical details and software functions, as well as algorithms characterizing the nonobviousness of these solutions in combination with the general principles of use of elements of artificial intelligence and artificial neural networks are also reflected.

\section{DETAILED DESCRIPTION}

[0050] According to some embodiments, an..., - this section describes the elements of essential novelty contained in the proposed invention at the level of the basic engineering solution of the top position in the hierarchy of new or updated engineering solutions and their integrative combinations.

[0051] In some embodiments, a..., - this section describes the principles of integration of software solutions into the basic engineering solution.

[0052] In order to develop..., - this section describes the fundamental elements of development of all types of engineering solutions at the level of the basic engineering solution without integration with software and test solutions.

[0053] To develop a..., - this section describes methods and processes, as well as algorithms designed for deep integration of software solutions of all kinds as a whole and hierarchy of the basic engineering solution and its integrative combinations with engineering subsystems. 
[0054] It will be appreciated that some..., - this section shows the reference and consequence of the input of software solutions in the principal characteristic and connections in all interconnected and mutually integrated engineering systems, taking into account the essential elements of artificial intelligence and related artificial neural networks.

[0055] In some embodiments, the..., - this section describes characteristics and new distinctive features that appeared during integration of software solutions of all levels into the basic engineering solution.

[0056] It will be appreciated that the..., - this section shows the elements of essential novelty that appeared during integration of software solutions into classic-type engineering solutions of all levels of the hierarchy.

[0057] In some embodiments, the..., - this section details characteristics and working relations of artificial intelligence elements with the basic scheme of operation of engineering systems of all levels of the hierarchy with reference of the principles of essential novelty to the algorithm or principles of operation of the entire complex invented engineering system.

[0058] In some embodiments, an..., - this section details characteristics and working relations of elements of artificial intelligence and artificial neural networks with the basic scheme of operation of engineering systems of all levels of the hierarchy with reference of the principles of essential novelty to the algorithm or principles of operation of the entire complex invented engineering system.

[0059] Reference will now be made to the figures, in which like numerals refer to like parts throughout.

[0060] In Figure 1..., - this section provides a detailed description of Figure 1 with a legend of all reference characters and reference numerals or digital designations. All digital designations for this figure should be of the form - 101, 102... The following reference numerals identify the following features.

[0061] Figure 2 shows an example of a..., - this section provides a detailed description of Figure 2 with a legend of all reference characters and reference numerals or digital designations. All digital designations for this figure should be of the form - 201, 202... The following reference numerals identify the following features.

[0062] Figure 3 shows a..., - this section provides a detailed description of Figure 3 with a legend of all reference characters and reference numerals or digital designations. All digital designations for this figure should be of the form - 301, 302... The following reference numerals identify the following features.

[0063] Figure 4 shows a..., - this section provides a detailed description of Figure 4 with a legend of all reference characters and reference numerals or digital designations. All digital 
designations for this figure should be of the form - 401, 402... The following reference numerals identify the following features.

[0064] Figure 5 shows a..., - this section provides a detailed description of Figure 5 with a legend of all reference characters and reference numerals or digital designations. All digital designations for this figure should be of the form - 501, 502... The following reference numerals identify the following features.

[0065] Figure 6 shows a..., - this section provides a detailed description of Figure 6 with a legend of all reference characters and reference numerals or digital designations. All digital designations for this figure should be of the form - 601, 602... The following reference numerals identify the following features.

[0066] Figure 7 shows..., - this section provides a detailed description of Figure 7 with a legend of all reference characters and reference numerals or digital designations. All digital designations for this figure should be of the form - 701, 702... The following reference numerals identify the following features.

[0067] Figure 8 shows an..., - this section provides a detailed description of Figure 8 with a legend of all reference characters and reference numerals or digital designations. All digital

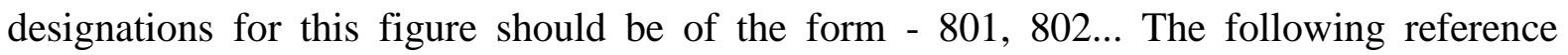
numerals identify the following features.

[0068] Figure 9 shows a..., - this section provides a detailed description of Figure 9 with a legend of all reference characters and reference numerals or digital designations. All digital designations for this figure should be of the form - 901, 902... The following reference numerals identify the following features.

[0069] Figure 10 shows a..., - this section provides a detailed description of Figure 10 with a legend of all reference characters and reference numerals or digital designations. All digital designations for this figure should be of the form - 1001, 1002... The following reference numerals identify the following features.

[0070] Figure 11 shows..., - this section provides a detailed description of Figure 11 with a legend of all reference characters and reference numerals or digital designations. All digital designations for this figure should be of the form - 1001, 1002... The following reference numerals identify the following features.

[0071] Figure 12 shows ..., - this section provides a detailed description of Figure 12 with a legend of all reference characters and reference numerals or digital designations. All digital designations for this figure should be of the form - 1201, 1202... The following reference numerals identify the following features. 
[0072] Figure 13 shows a..., - this section provides a detailed description of Figure 13 with a legend of all reference characters and reference numerals or digital designations. All digital designations for this figure should be of the form - 1301, 1302... The following reference numerals identify the following features.

[0073] Figure 14 shows..., - this section provides a detailed description of Figure 14 with a legend of all reference characters and reference numerals or digital designations. All digital designations for this figure should be of the form - 1401, 1402... The following reference numerals identify the following features.

[0074] Figure 15 shows a..., - this section provides a detailed description of Figure 15 with a legend of all reference characters and reference numerals or digital designations. All digital designations for this figure should be of the form $-1501,1502 \ldots$ The following reference numerals identify the following features.

[0075] Figure 16 shows a..., - this section provides a detailed description of Figure 16 with a legend of all reference characters and reference numerals or digital designations. All digital designations for this figure should be of the form - 1601, 1602... The following reference numerals identify the following features.

[0076] Figure 17 shows..., - this section provides a detailed description of Figure 17 with a legend of all reference characters and reference numerals or digital designations. All digital designations for this figure should be of the form - 1701, 1702... The following reference numerals identify the following features.

[0077] Figure 18 shows a..., - this section provides a detailed description of Figure 18 with a legend of all reference characters and reference numerals or digital designations. All digital designations for this figure should be of the form - 1801, 1802... The following reference numerals identify the following features.

[0078] Figure 19 shows a..., - this section provides a detailed description of Figure 19 with a legend of all reference characters and reference numerals or digital designations. All digital designations for this figure should be of the form - 1901, 1902... The following reference numerals identify the following features.

[0079] Figure 20 shows..., - this section provides a detailed description of Figure 20 with a legend of all reference characters and reference numerals or digital designations. All digital designations for this figure should be of the form - 2001, 2002... The following reference numerals identify the following features.

[0080] Figure 21 shows a..., - this section provides a detailed description of Figure 21 with a legend of all reference characters and reference numerals or digital designations. All digital 
designations for this figure should be of the form - 2101, 2102... The following reference numerals identify the following features.

[0081] Figure 22 shows an..., - this section provides a detailed description of Figure 22 with a legend of all reference characters and reference numerals or digital designations. All digital designations for this figure should be of the form - 2201, 2202... The following reference numerals identify the following features.

[0082] Figure 23 shows a..., - this section provides a detailed description of Figure 23 with a legend of all reference characters and reference numerals or digital designations. All digital designations for this figure should be of the form - 2301, 2302... The following reference numerals identify the following features.

[0083] Figure 24 shows a..., - this section provides a detailed description of Figure 24 with a legend of all reference characters and reference numerals or digital designations.

\section{TEST RESULTS}

[0084]This section demonstrates the test results in the form of indicators, tables, graphs, and charts.

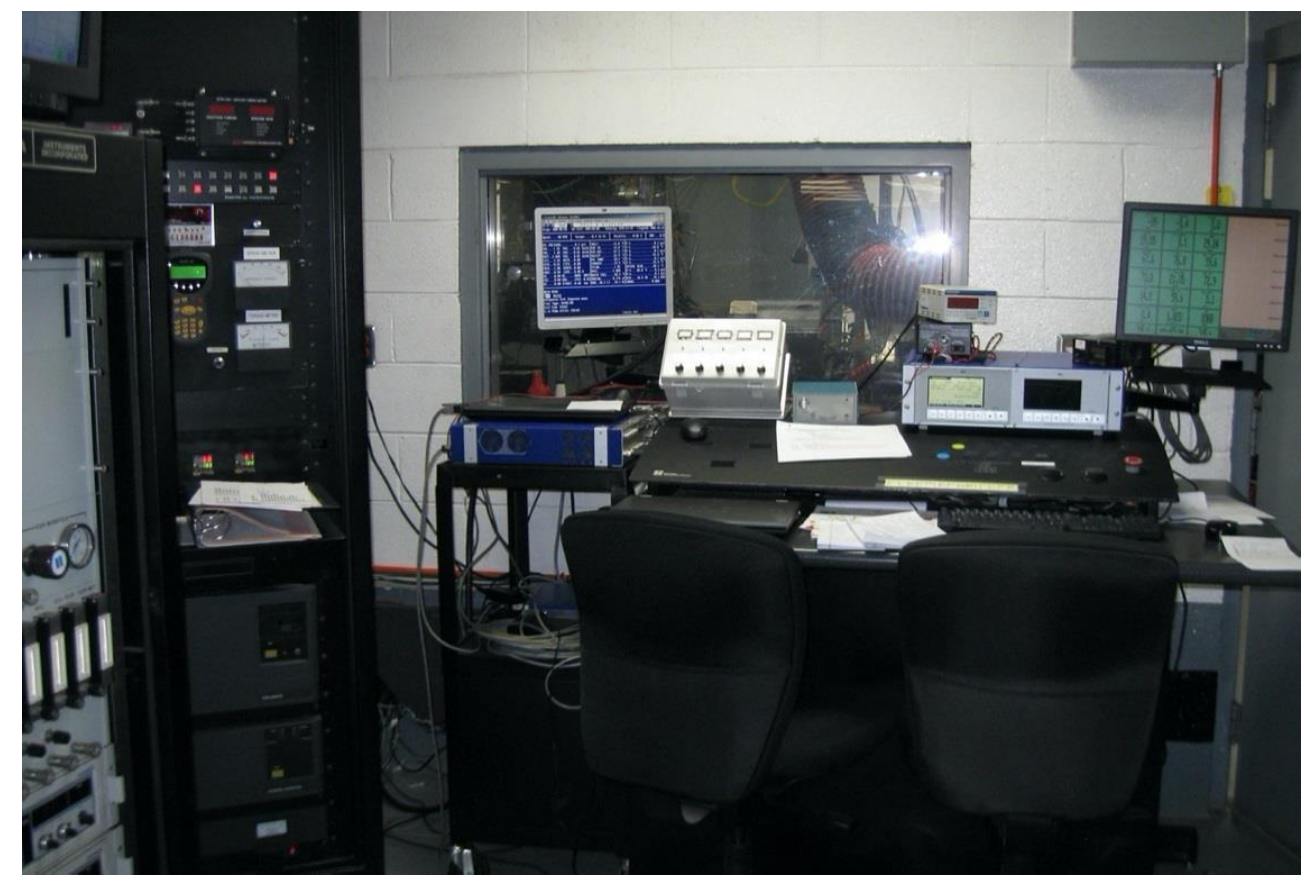

Figure 1, - the figure shows an example of the location of the system of analytical and control equipment of the test bench, intended for testing and analytical evaluation of a diesel engine. Bench systems include elements of artificial intelligence and artificial neural networks. It is very important to show and reveal the novelty of using this system to achieve an ideal end result or its equivalent in relation to the claimed material 


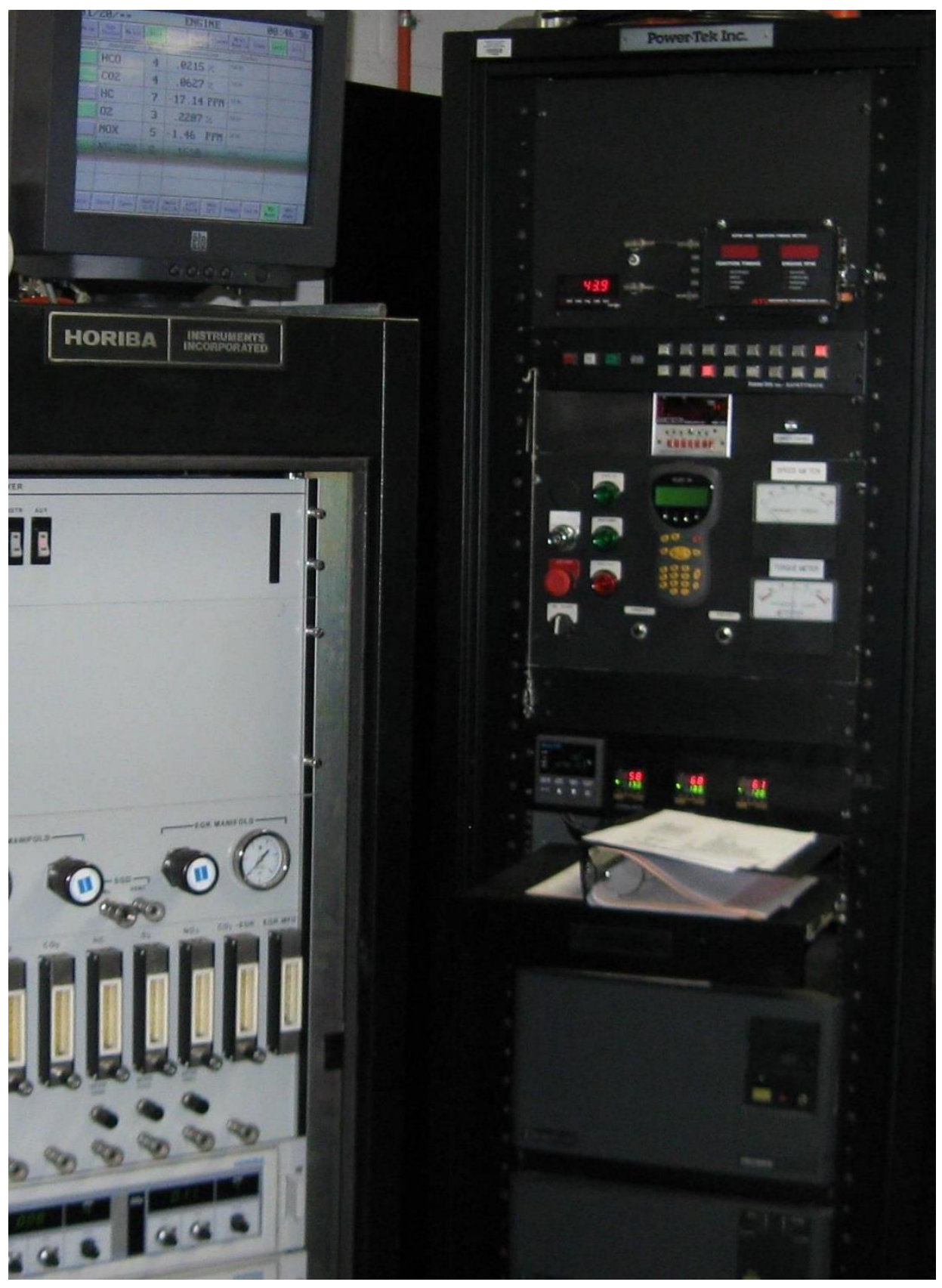

Figure 2, - the figure shows an example of a fragment of the location of the system of analytical and control equipment of the test bench, intended for testing and analytical evaluation of a diesel engine. Bench systems include elements of artificial intelligence and artificial neural networks. It is very important to show and disclose the novelty of using this system to achieve an ideal end result or its equivalent in relation to the claimed material, as well as to show the interaction of the control and analytical components of the system with elements of artificial intelligence 


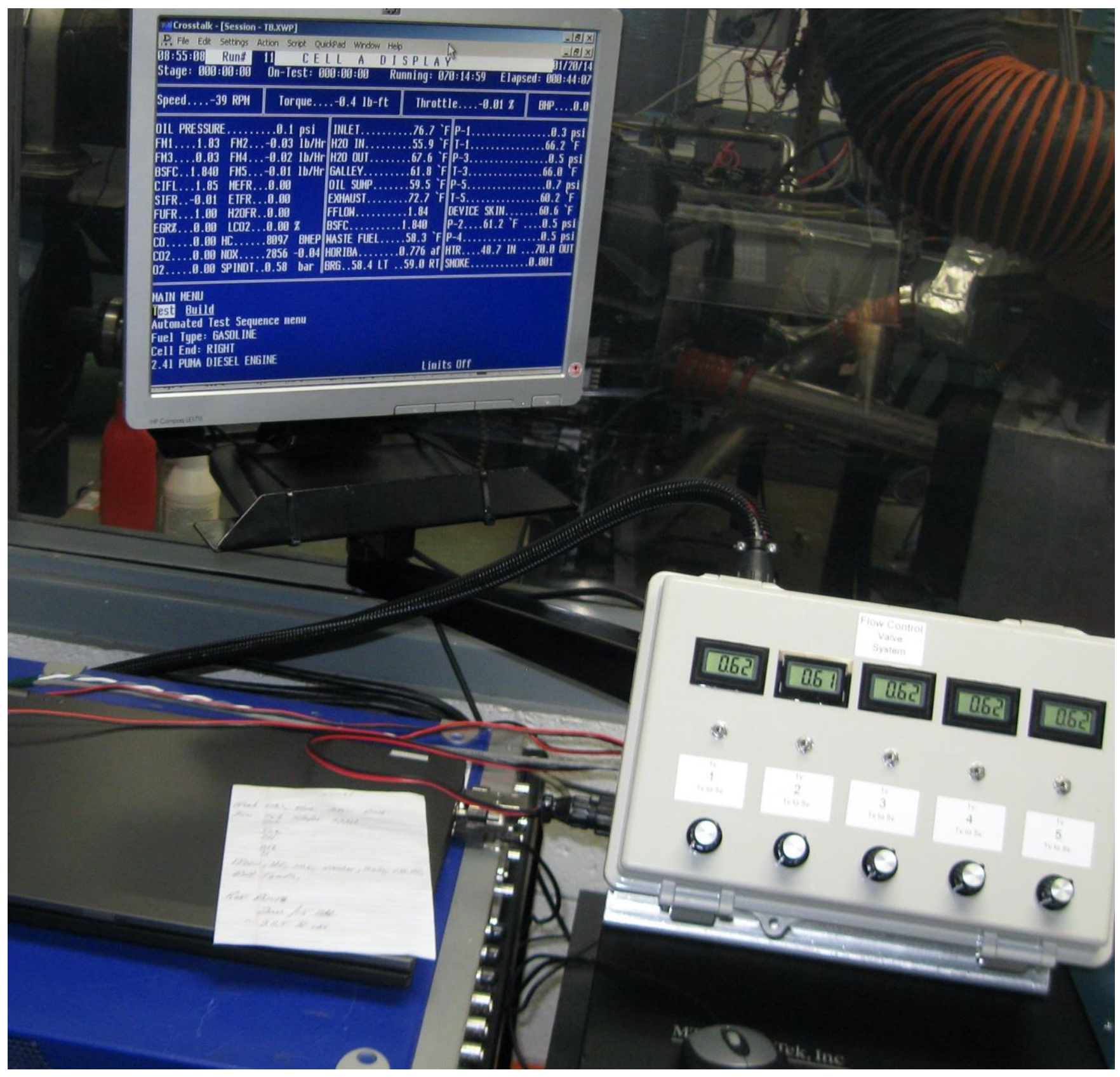

Figure 3, - the figure shows an example of the location of the system of analytical and control equipment of the test bench, intended for testing and analytical evaluation of a diesel engine. Bench systems include elements of artificial intelligence and artificial neural networks. It is very important in the application materials to show and disclose the novelty of the application of this system to achieve an ideal end result or its equivalent in relation to the claimed material 


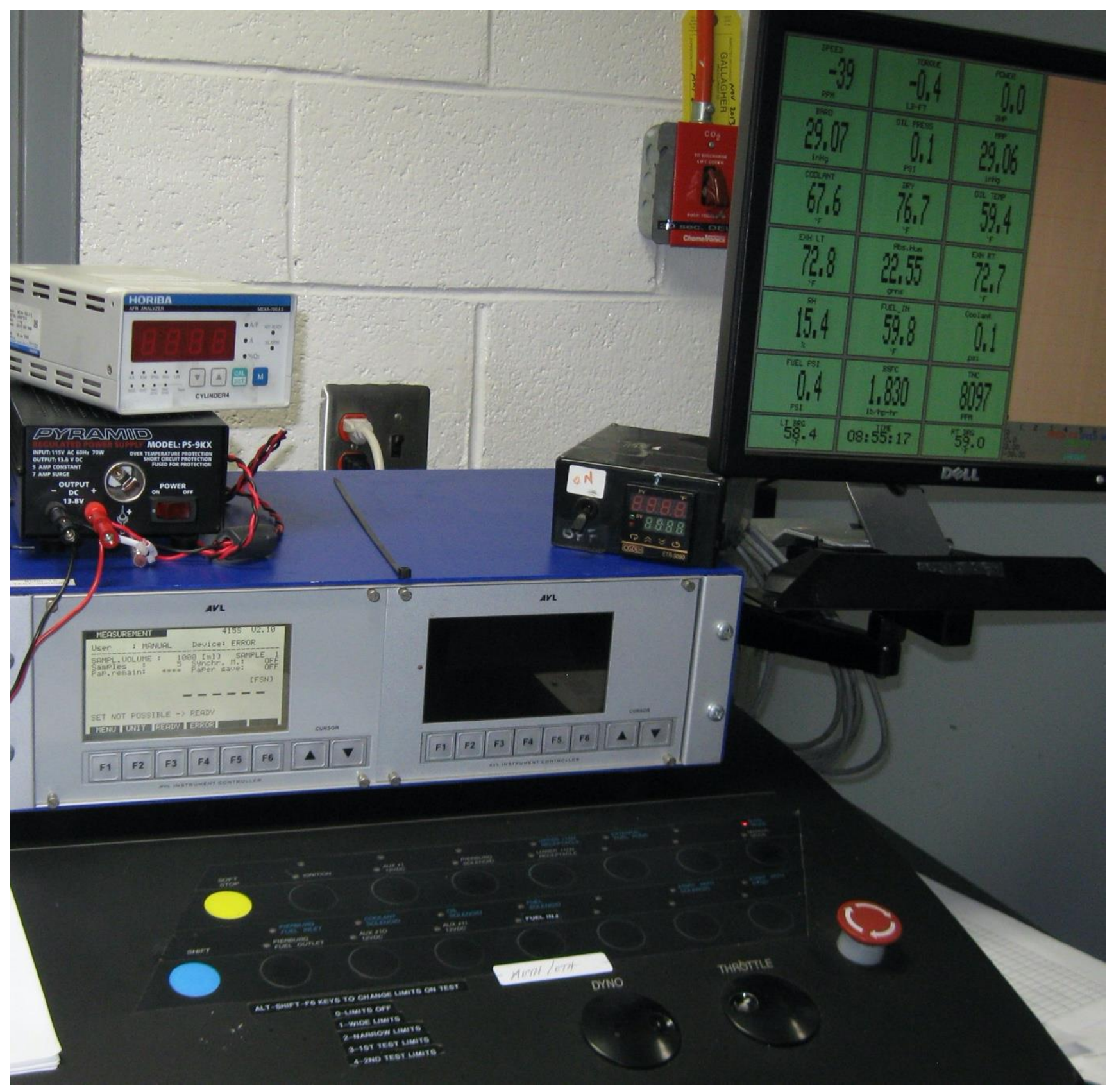

Figure 4, - the figure shows an example of a fragment of the location of the system of analytical and control equipment of the test bench, intended for testing and analytical evaluation of a diesel engine. Bench systems include elements of artificial intelligence and artificial neural networks. It is very important to show and disclose the novelty of using this system to achieve an ideal end result or its equivalent in relation to the claimed material, as well as to show the interaction of the control and analytical components of the system with elements of artificial intelligence 

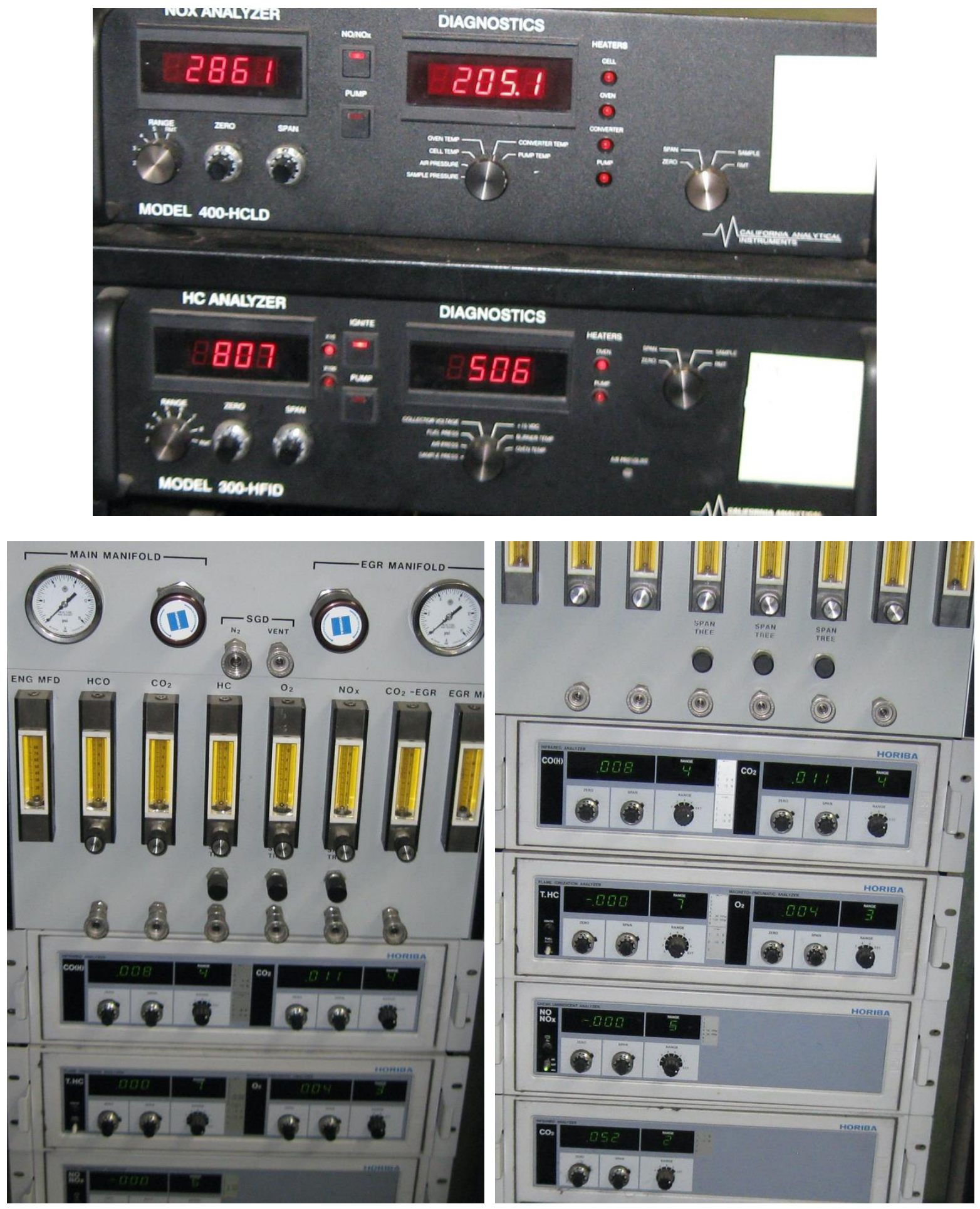

Figures 5, 6, 7, - the figures show examples of fragments of the location of the system of analytical and control equipment of the test bench, intended for testing and analytical evaluation of a diesel engine. It is very important to show and reveal the novelty of the application of this system, including the control and analytical components of the system with elements of artificial intelligence 


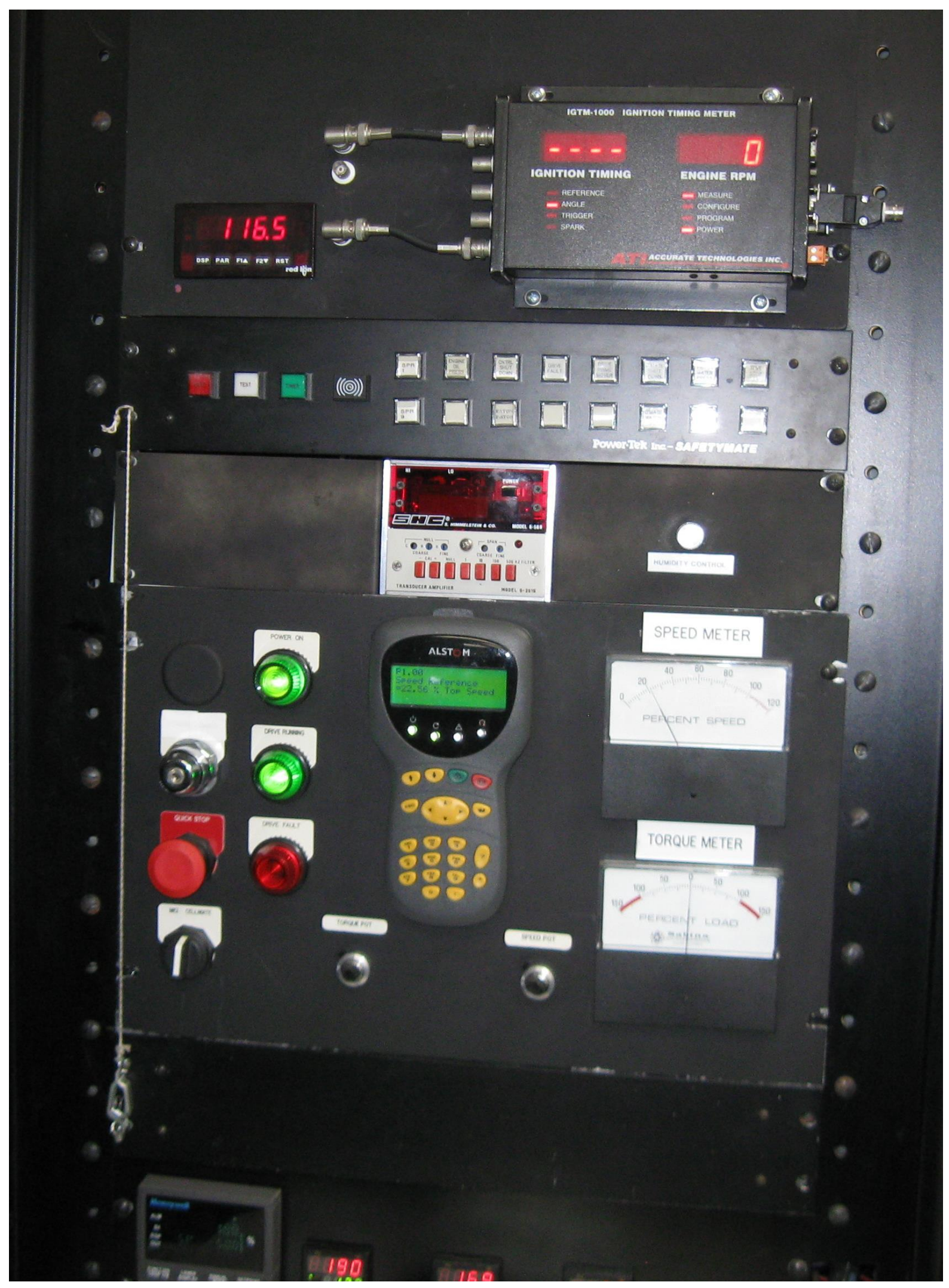

Figure 8 
Figures 8, 9, 10 and 11 show examples of fragments of the location of the system of analytical and control equipment of the test bench, intended for testing and analytical evaluation of a diesel engine. It is very important to show and reveal the novelty of the application of this system, including the control and analytical components of the system with elements of artificial intelligence.

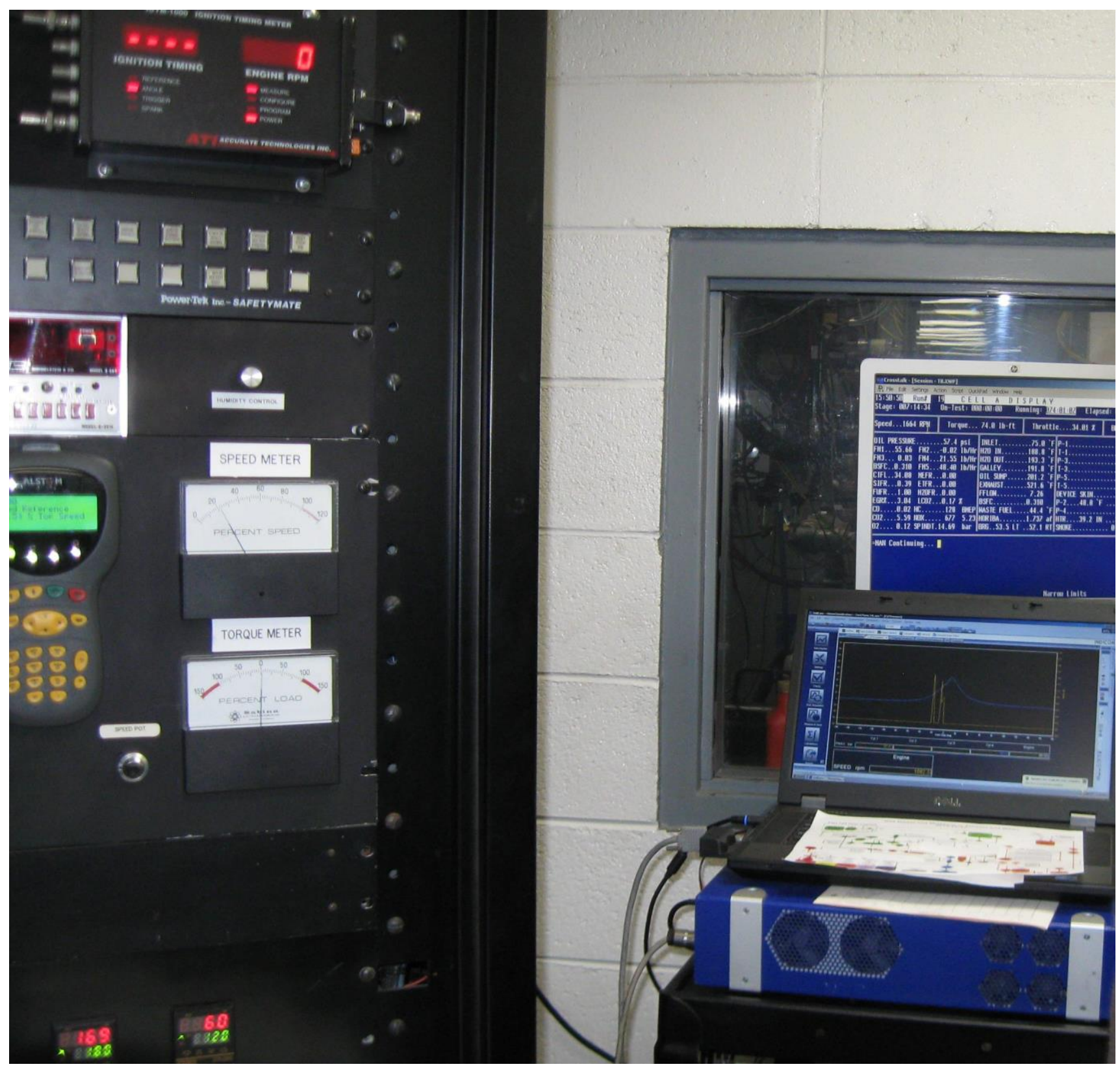

Figure 9 


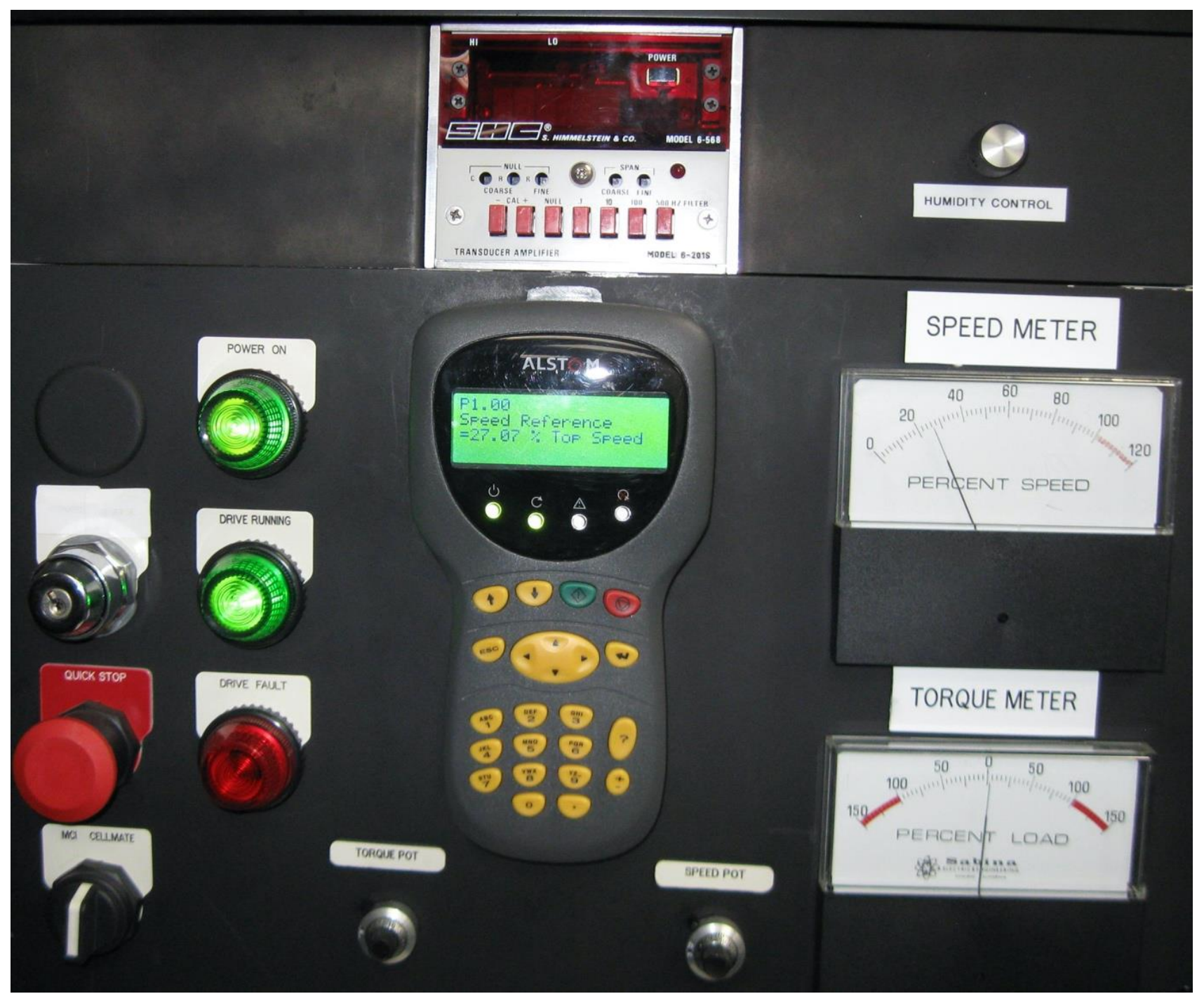

Figure 10, - the figure shows, as an example, the components of a control and analytical system that allow, in the process of comparative evolution of measurement results and comprehensive monitoring of the whole complex of technological transitions, to use the capabilities and characteristics of elements of artificial intelligence and artificial neural networks

This is especially important in the constant coordination of the main operating parameters of the test bench and the response from these parameters to the test components of the engine installed on the dynamometer table of the bench.

In the section in which the test results are given, it is necessary to connect the novelty elements of the modified engine components with the determination of their real indicators and the determination of the effect of this novelty on the nature of the indicators. 


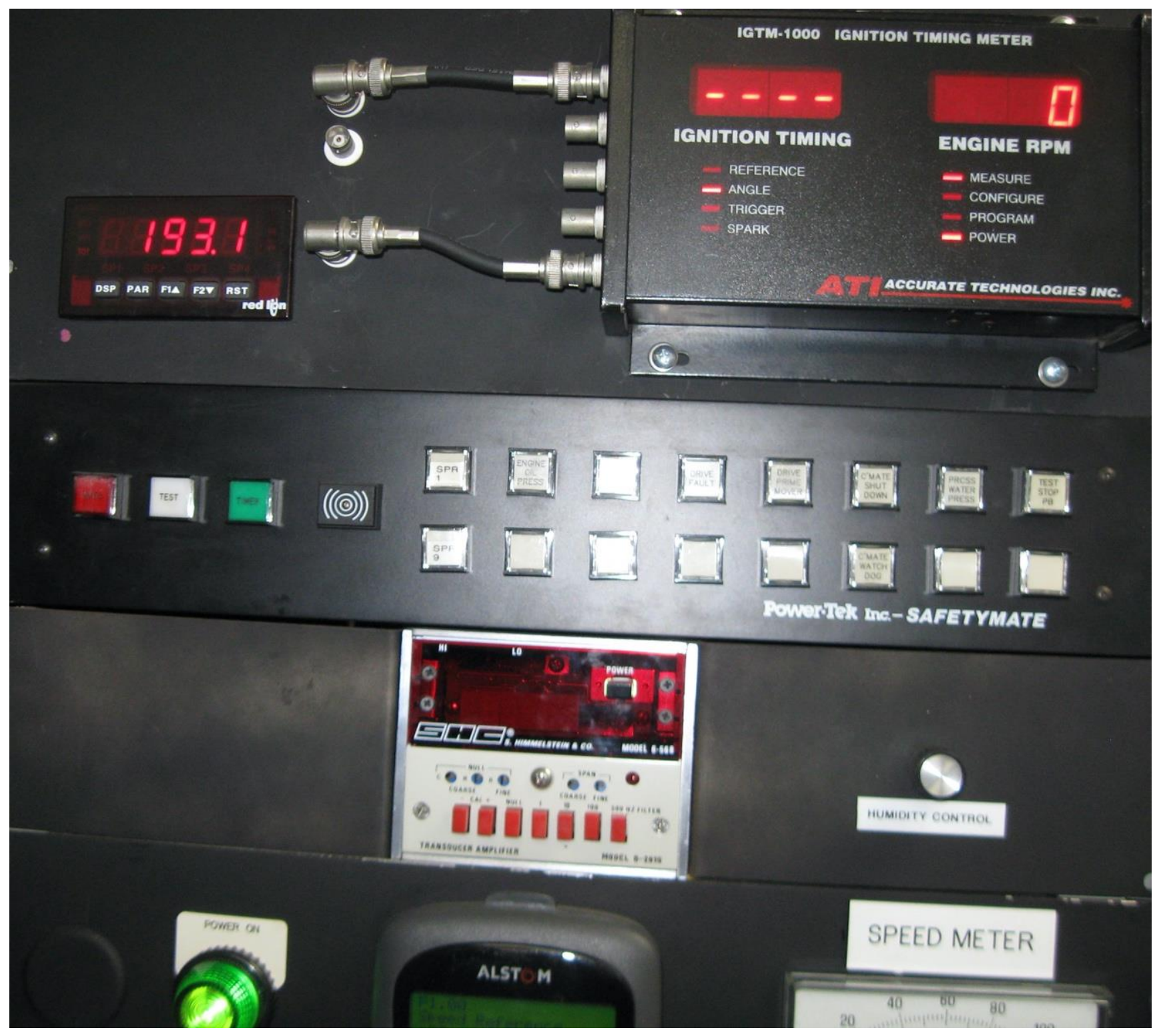

Figure 11, - the figure also shows, as an example, the components of the control and analytical system that allow the capabilities and characteristics of artificial intelligence elements and artificial neural networks to be used in the process of comparative evolution of measurement results and integrated monitoring of the entire complex of technological transitions

In the section in which the test results are given, it is necessary to connect the novelty elements of the modified engine components with the determination of their real indicators and the determination of the effect of this novelty on the nature of the indicators. 


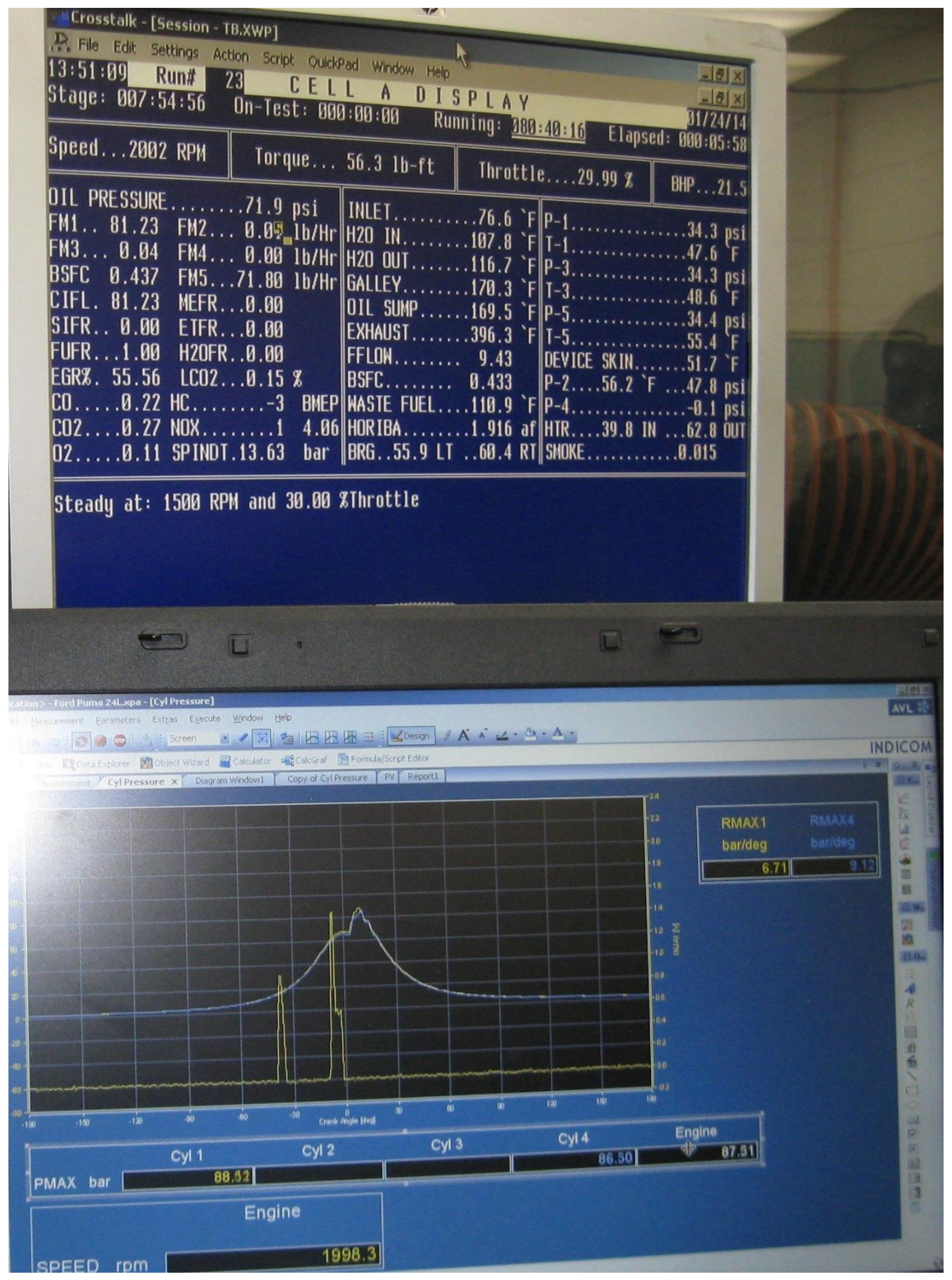


Figure 12

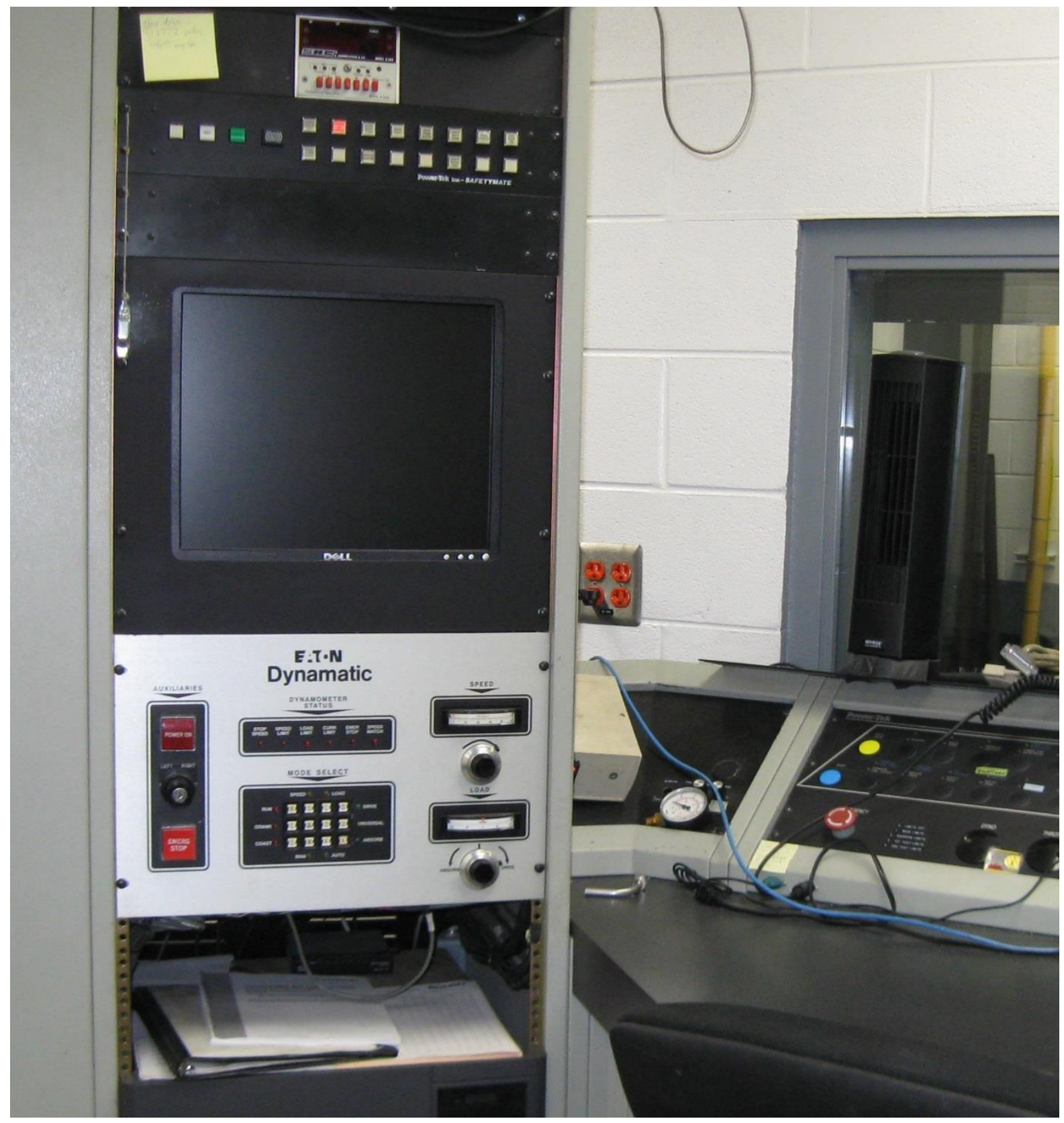

Figure 13

Figures 12, 13, 14, and 15 show the control and measurement processes that determine the nature of the interaction between various devices and measurement systems and their coordination with elements of artificial intelligence and artificial neural networks in terms of optimizing the main parameters of the technical characteristics of the engine, including environmental indicators associated with with the formation of fuel mixtures, including methanol or biological fuels. 


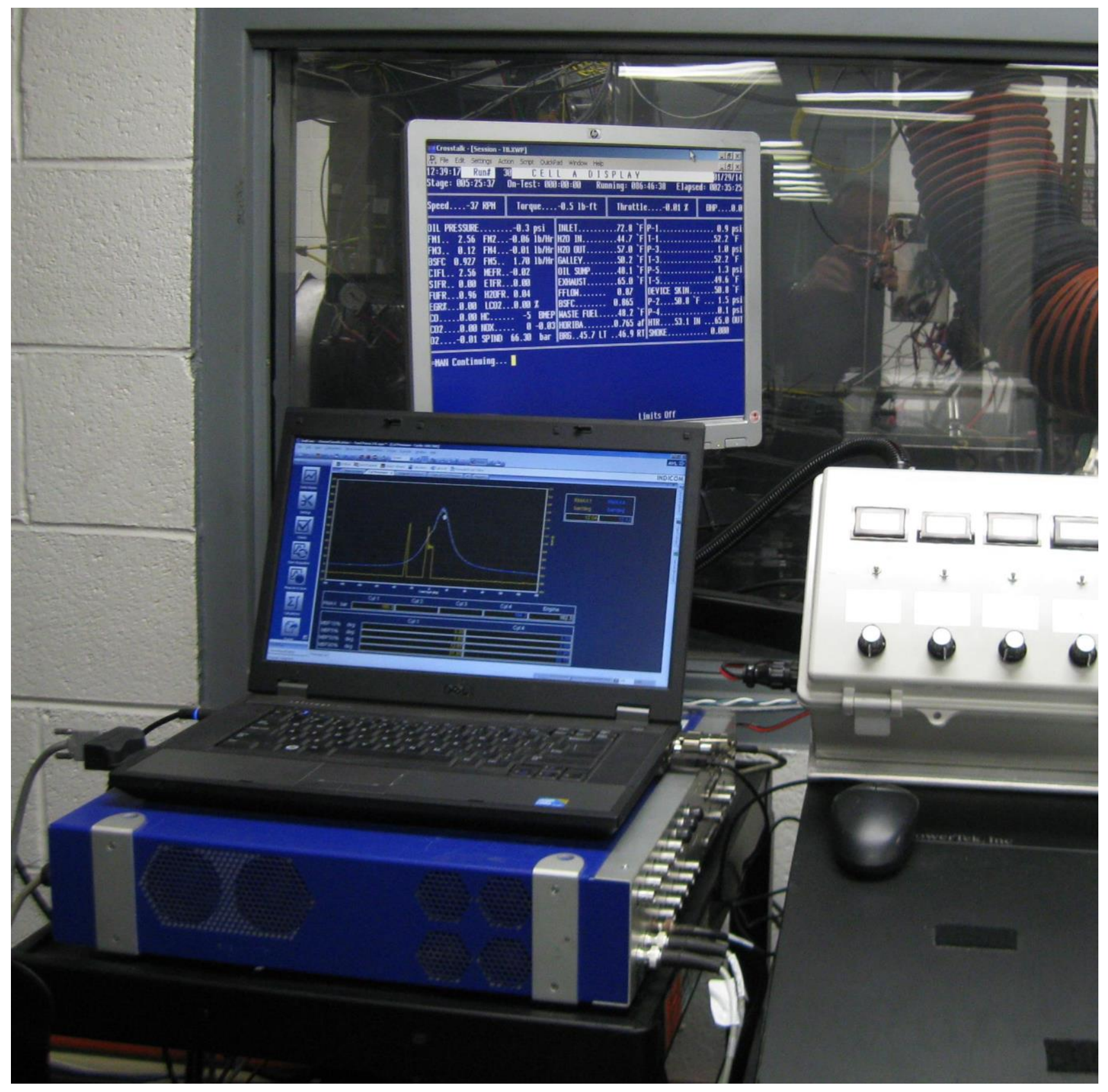

Figure 14

For applications for the proposed invention, it is important to show the influence of elements and signs of significant novelty of the proposed technical solution in their active combination with elements of artificial intelligence and artificial neural networks in combination with an ideal end result aimed at obtaining an unusual test result that does not take place in known technical solutions and characterizing the proposed technical solution. 


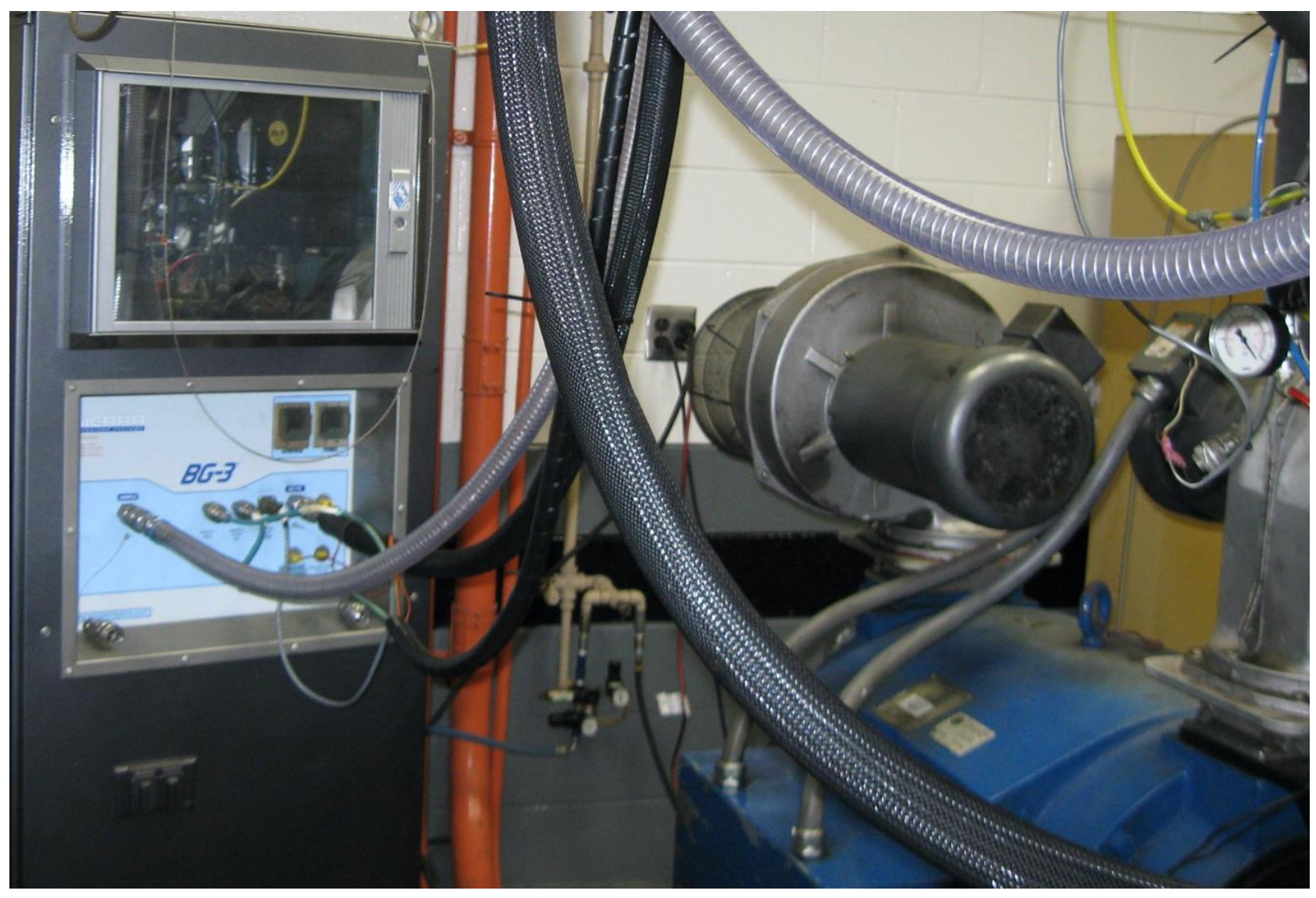

Figure 15






\section{Figure 16}

Figure 16 shows the engine on the stand, which controls more than 1200 parameters, the processing of the measurement results of which is carried out in real time using elements of artificial intelligence and artificial neural networks.

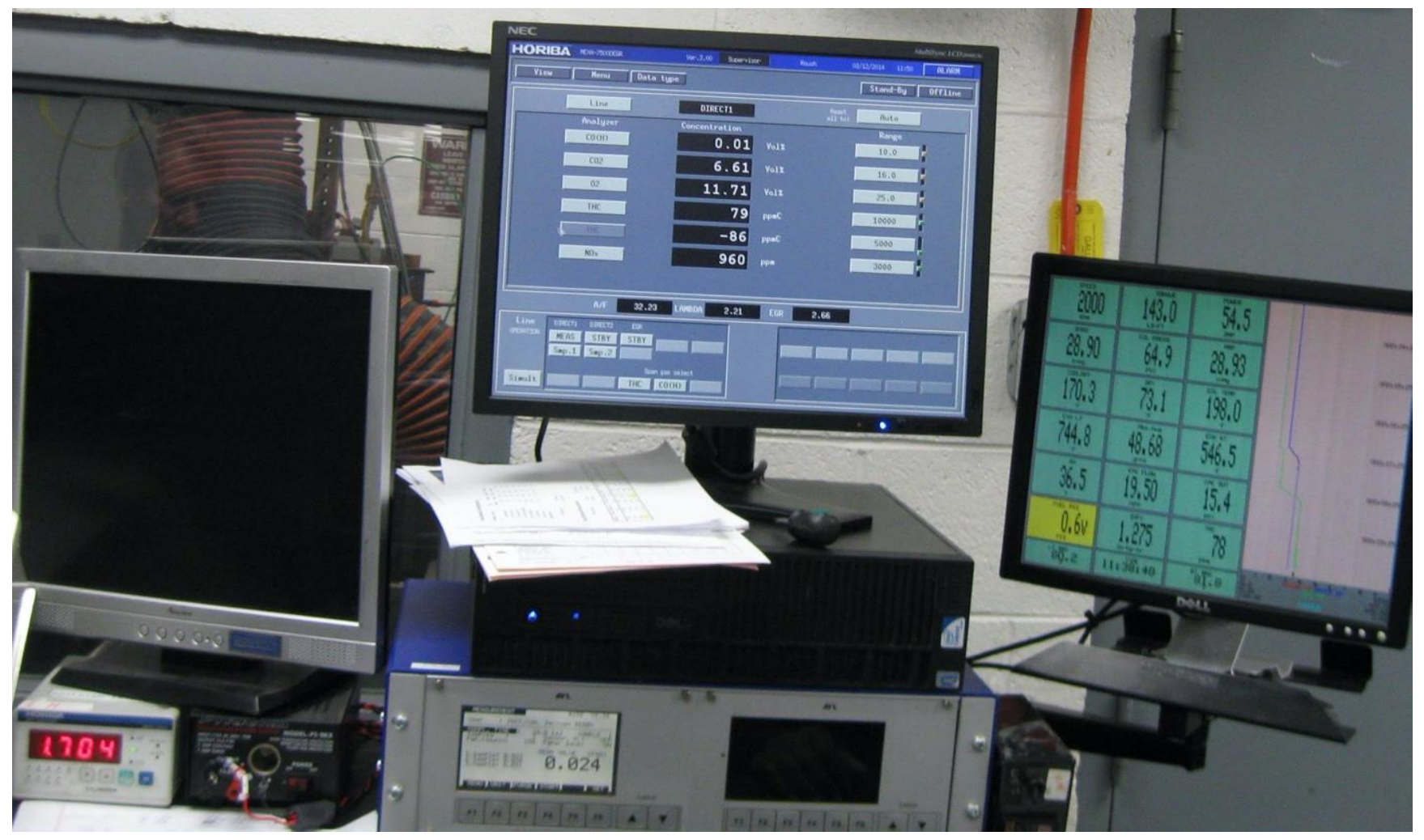

Figure 17, - the figure shows, as an example, the components of a control and analytical system that allow, in the process of comparative evolution of measurement results and comprehensive monitoring of the whole complex of technological transitions, to use the capabilities and characteristics of elements of artificial intelligence and artificial neural networks

This is especially important in the constant coordination of the main operating parameters of the test bench and the response from these parameters to the test components of the engine installed on the dynamometer table of the bench.

In the section in which the test results are given, it is necessary to connect the novelty elements of the modified engine components with the determination of their real indicators and the determination of the effect of this novelty on the nature of the indicators. 


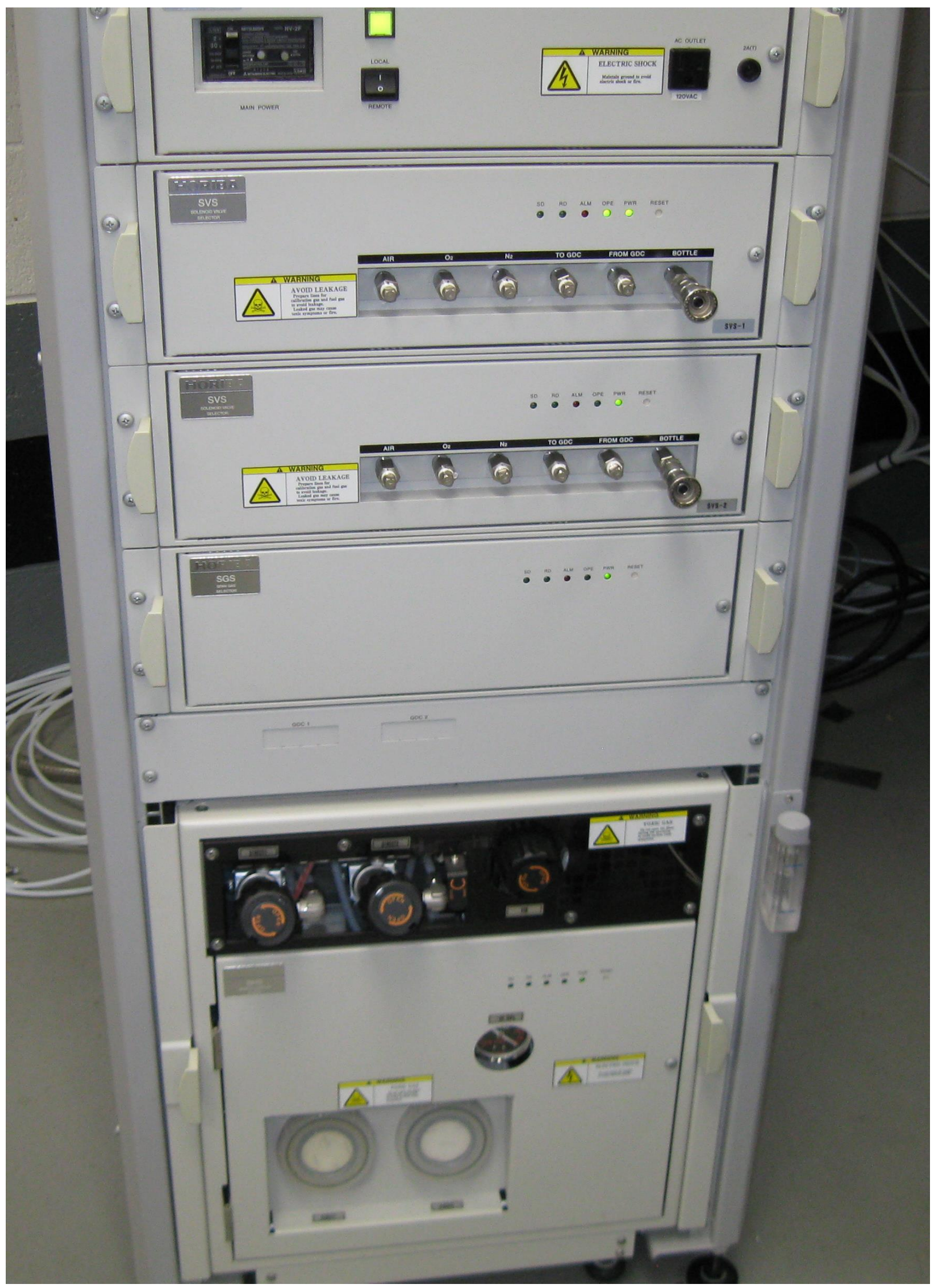




\section{Figure 18}

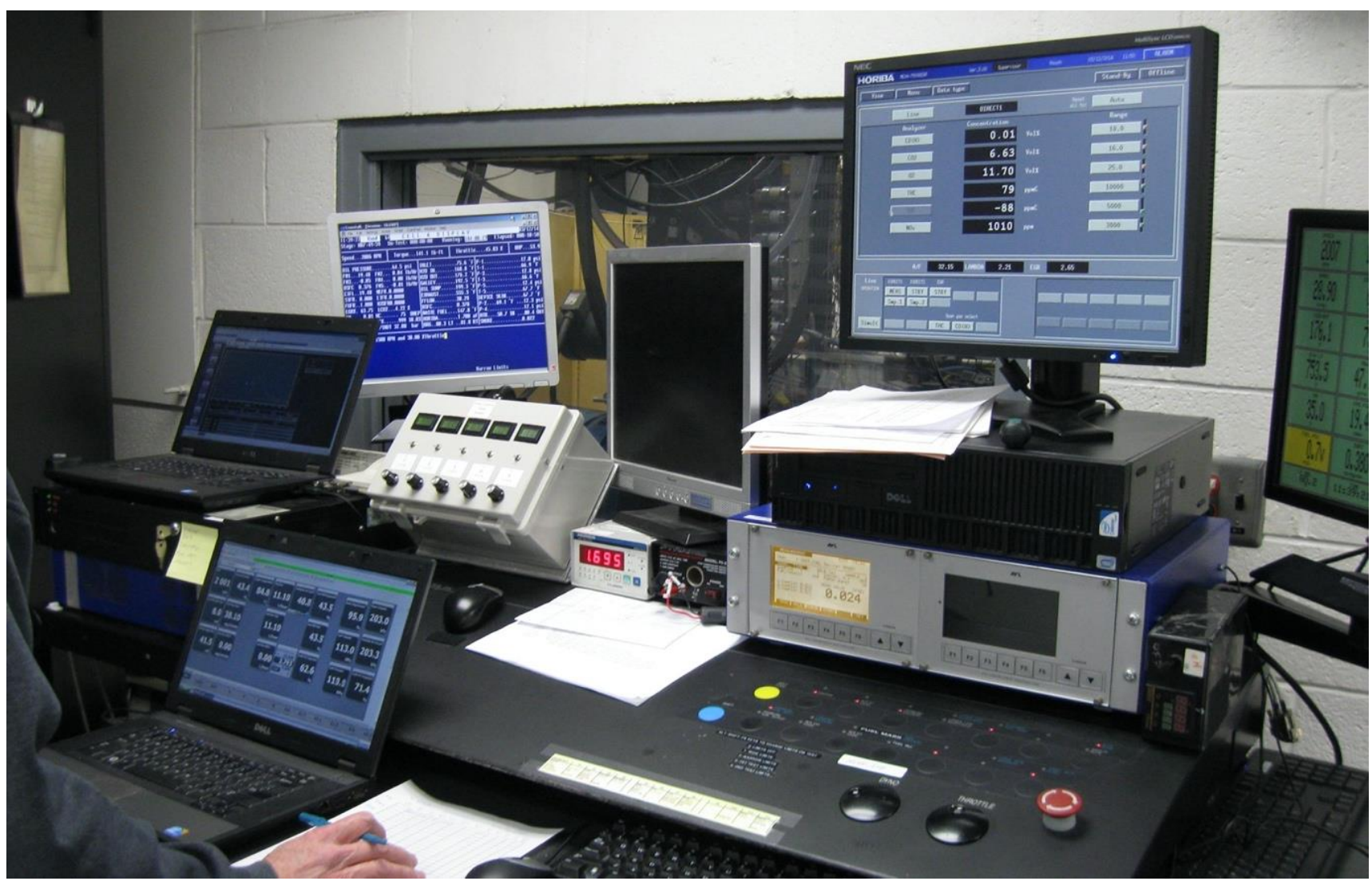

Figure 19

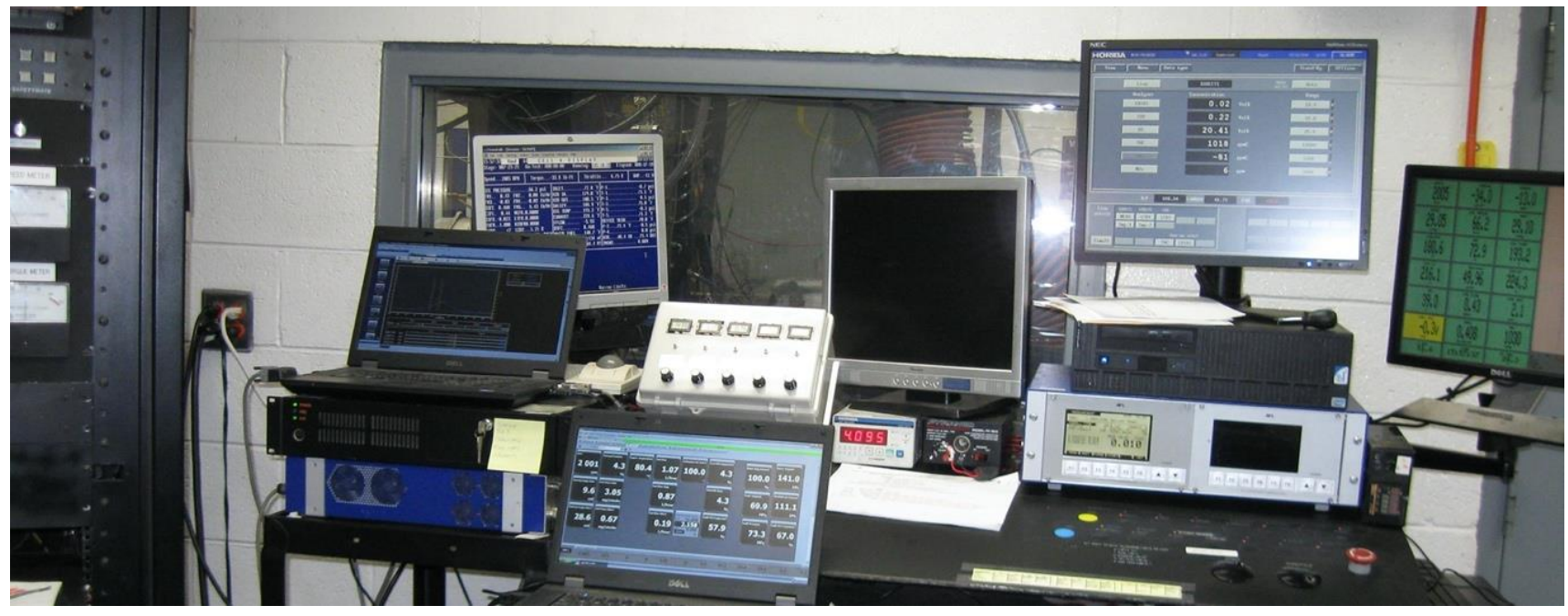

Figure 20 


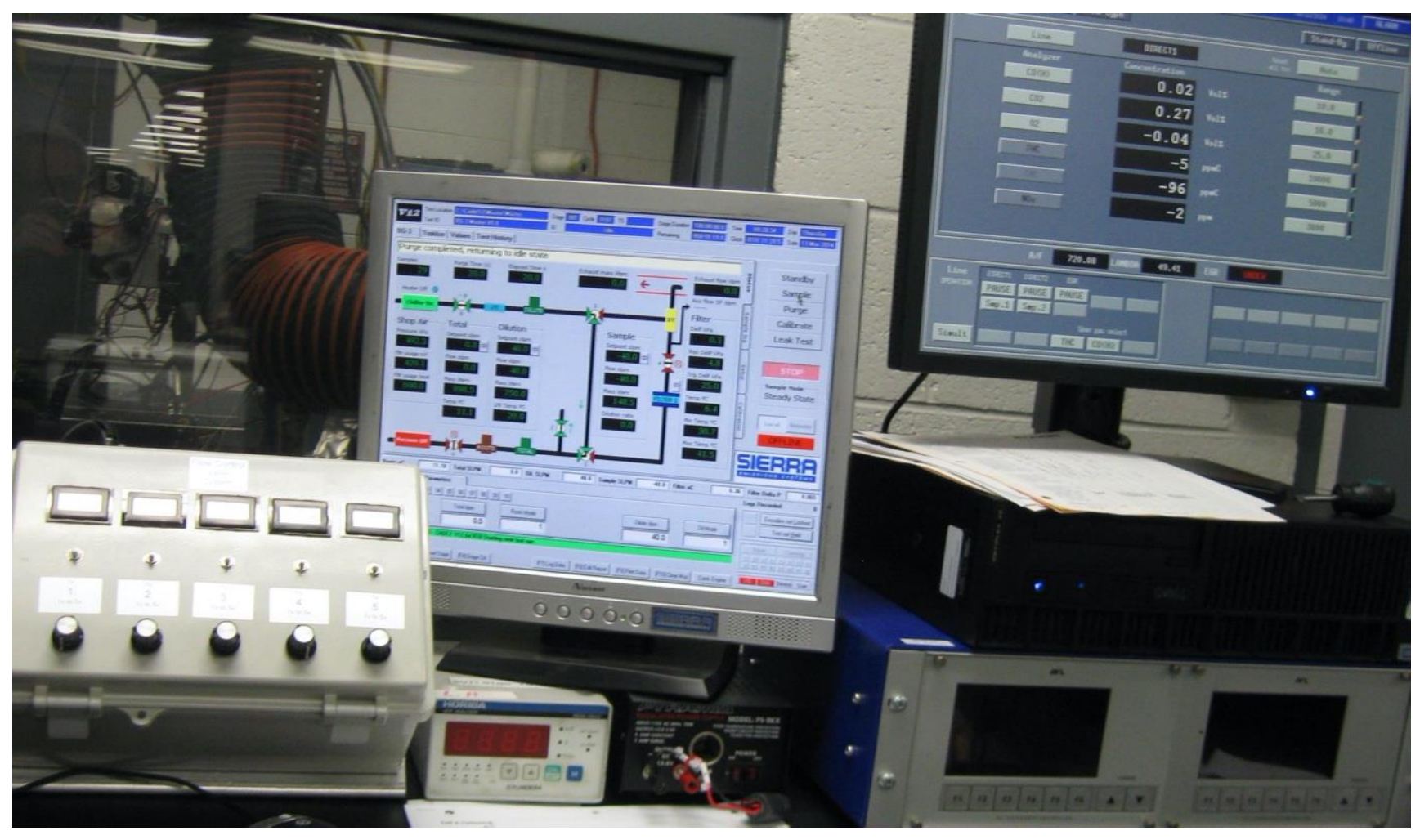

Figure 21

Figures 18, 19, 20, 21, 22, 23, and 24, - also show, as an example, the components of a controlanalytical system that are functionally connected with each other and simultaneously with elements of artificial intelligence and artificial neural networks, allowing in the process of comparative evolution of measurement results and integrated monitoring of the whole complex of technological transitions also comprehensively apply the capabilities and characteristics of elements of artificial intelligence and artificial neural networks.

This is especially important in the constant coordination of the main operating parameters of the test bench and the response from these parameters to the test components of the engine installed on the dynamometer table of the bench.

In the section in which the test results are given, it is necessary to connect the novelty elements of the modified engine components with the determination of their real indicators and the determination of the effect of this novelty on the nature of the indicators. 


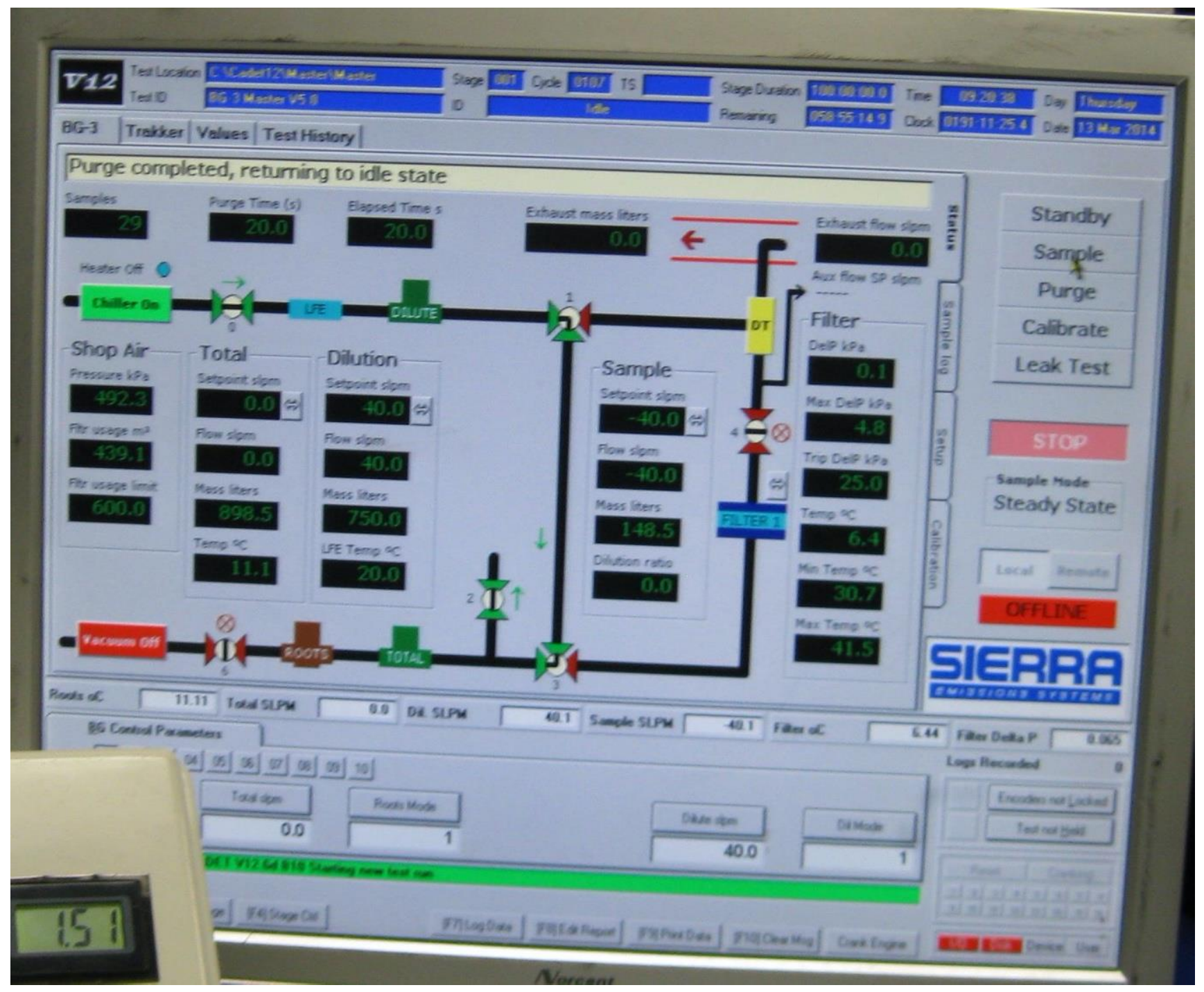

Figure 22

For the given example, indicators and combinations of measurement results of various, interconnected engine operation parameters, which vary depending on the factor that affects the result to the maximum extent, are typical, moreover, this may be the composition of the fuel mixture, including only liquid components, or liquid and gaseous components or only gaseous components.

A constant analysis of the effectiveness of all elements of novelty and their influence on the degree or level of achievement of an ideal end result to the maximum extent depends on the inclusion or not inclusion in the control and analytical complex of elements of artificial intelligence and artificial neural networks. 


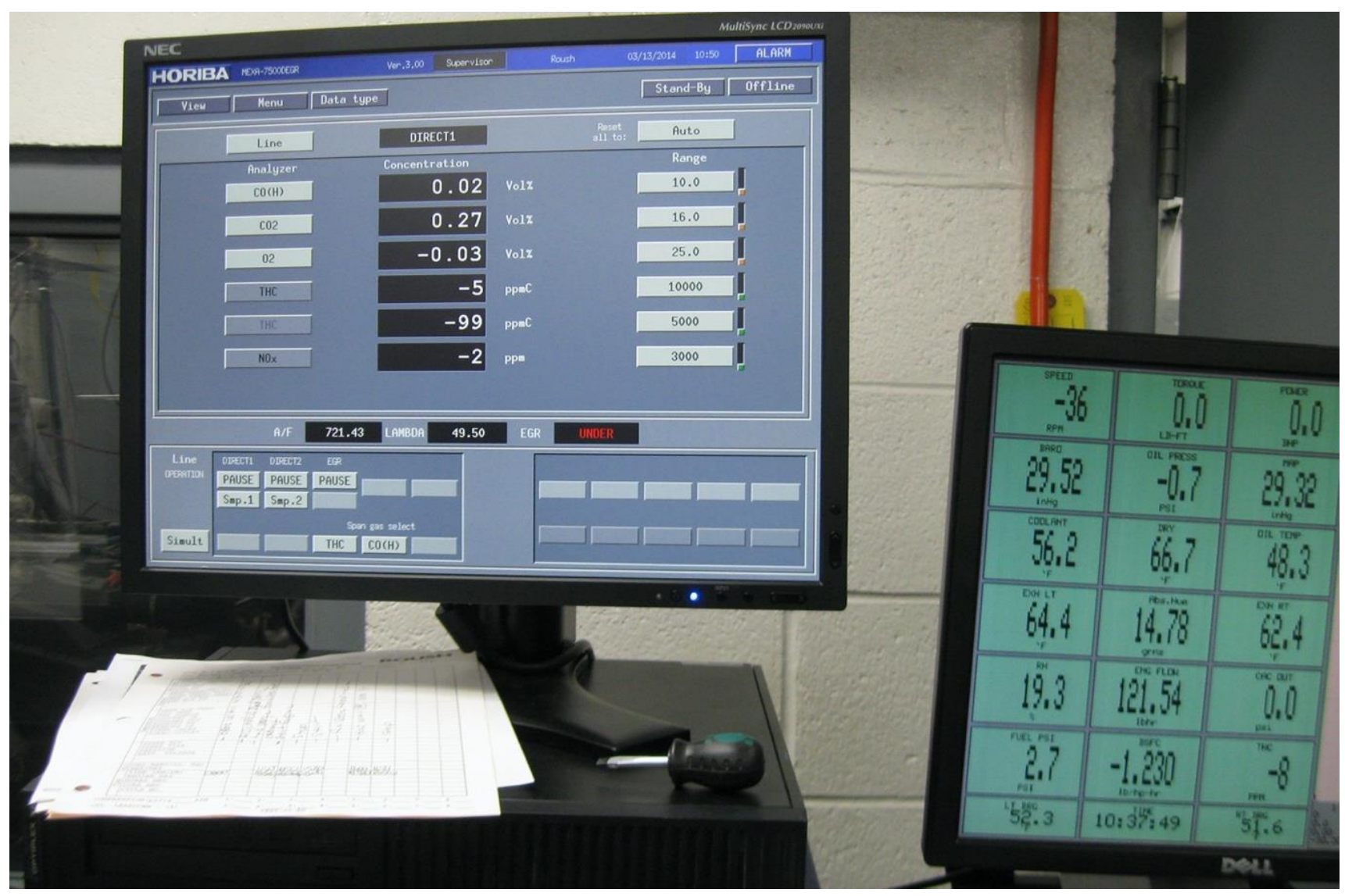

Figure 23

The task of inclusion and a detailed analysis of the impact of this inclusion is the material that should be included in the description of the application for the invention, in the section of evidence of the effectiveness of the elements of significant novelty and the results of their comprehensive tests using the above equipment.

In the text of the application related to the description of the test process and characterization of the test results, it is necessary to give a detailed detailed explanation of the principles of constructing the test process in combination with the analytical processing of the test results, including the connection with the requirements of the standards in force during the test period.

The description should include a characterization of the general distinguishing features of the alleged invention and their causal relationship with the results.

It is also necessary to indicate how new and non-obvious the results are. 


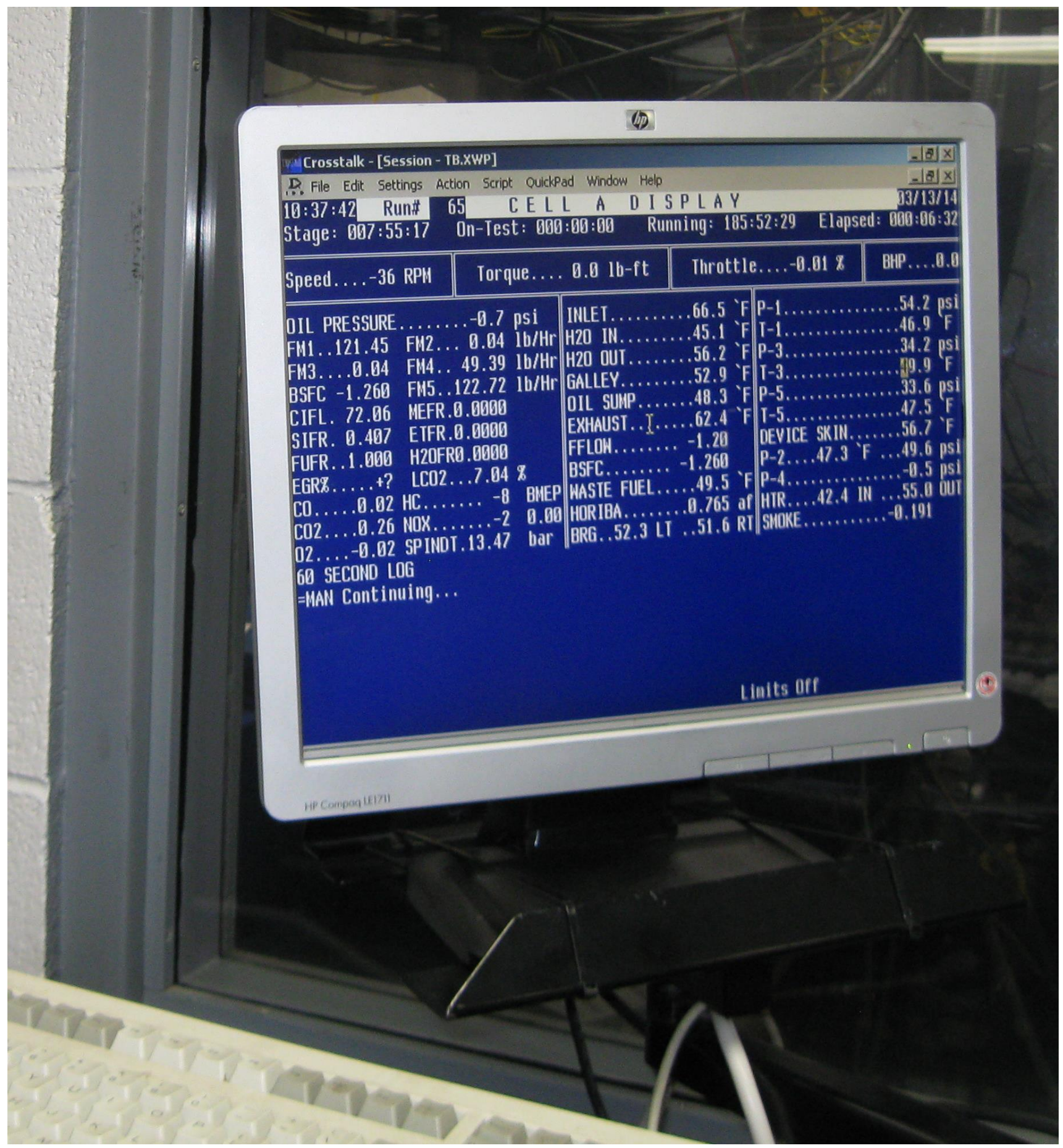

Figure 24

It is important to show the connection of the invention with smart technologies and the possibilities of combining software products of a new solution with the structural elements of an apparatus or system, forming a complex of new technical solutions that form the basis of the invention. 
[0085] Test results showed that in the..., - in this section, the test results are identified and compared with similar test results of known devices or apparatuses.

[0086] This section proves that the test results obtained correspond to the expected and declared indicators of the ideal final result.

\section{ANALYSIS OF THE TEST RESULTS}

[0087] Qualification test result analysis showed that the embodiments of the invention achieve the objective of..., - this section provides a structural analysis and characterization of the test results of prototypes of the invented engineering solution.

Next, you need to analyze the General distinguishing features of the invention, which distinguish it from known patent materials.

As an example, we consider an invention - an internal combustion engine, classified as a smart transport system for all structural elements and operating principles:

$\circ$ an internal combustion engine, into the fuel mixture supply and preparation system of which, a device for dynamically mixing liquid fuel with other components of the fuel mixture is integrated;

o the operating cycle of the device for dynamically mixing liquid fuel with other components of the fuel mixture occurs in the fuel pipeline with the main parameters of the fuel pipeline that occur in the normal duty cycle of any internal combustion engine;

$\circ$ in the operation of an engine with an integrated device for dynamically mixing the liquid component of the fuel mixture with other components of the fuel mixture, only materials and substances are used that are commonly used in the operation of serial internal combustion engines;

- a device for dynamic mixing of components has two complex input devices sequentially arranged along the course of the fuel, ending with vortex generators forming a vortex tube within the fuel pipeline;

o the device for dynamically mixing the components of the fuel mixture has a mixing system, which when mixing equalizes the level of turbulence in each cross section of the fuel pipeline in the center of the cross section of the pipeline and on its periphery;

$\circ$ the fuel mixture at the outlet of the device for dynamic mixing of the components of the fuel mixture, depending on the mixing ratio between the components, and depending on the nature and type of components, may have a different structure and composition:

○ emulsions

$\circ$ foamed emulsion

○ foamed fuel mixture 
○ fuel foam

○ aerosols

○ carbonated fuel.

In none of the known technical solutions does the fuel mixture have such a composition and structure before being injected into a cylinder or combustion chamber.

If gas acts as a component of the fuel mixture, due to the fact that in the device for dynamically mixing liquid fuel with other components of the fuel mixture, the contact surface area in the boundary layer — liquid / gas — increases by at least an order of magnitude compared with opposed technical solutions (at least 10 times) the concentration of dissolved gas in a liquid increases in proportion to the increase in contact area:

$\circ$ for mixing, only materials and components, both liquid and gaseous, which are traditionally used for the preparation of fuel mixtures, are used;

O for the preparation of fuel mixtures in the proposed technical solution, only known technological methods and actions are applied ..., only hydrodynamic and aerodynamic mixing and technological methods and actions are not applied, as in well-known patent applications:

○ "Fuel converter" - the declared effect is achieved due to heating, evaporation and catalysis of the fuel mixture, and not due to only hydrodynamic and aerodynamic mixing, as is the case in the proposed technical solution. In the known material there are no signs inherent in the technical solution underlying the present invention and, based on this, the known technical solution is not the technical equivalent of the claimed technical solution according to any of the signs and criteria.

○ "Heated catalyzed fuel injector for injection ignition engines", the declared effect is achieved due to additional compression and heating of the fuel mixture and additional catalytic effect on the fuel mixture, and not due to only hydrodynamic and aerodynamic mixing of the components of the fuel mixture, as in the proposed technical solution. In the known material there are no signs inherent in the technical solution underlying the present invention and, based on this, the known technical solution is not the technical equivalent of the claimed technical solution according to any of the signs and criteria.

○ "Single nozzle direct injection system for rapidly variable gasoline / anti-knock agents mixtures", the declared effect is completely different from the effect of the proposed technical solution. The goal of the well-known technical solution ..., is mixing an antiknock agent with the fuel mixture, which reduces the level of gasoline detonation. In this case, mixing is carried out according to a technology that is absolutely not equal and not equivalent in any way to the claimed technical solution; In the known material there are no signs inherent in the technical solution underlying the present invention, and, based on this, the 
opposed technical solution is not the technical equivalent of the claimed technical solution according to any of the signs and criteria.

○ "Internal combustion engine / water sours system", the declared effect is the cooling of exhaust gases in order to condense water from them, it is proposed to carry out by compressing the exhaust gases and forming a vortex tube from them. Based on the materials set forth in the well-known technical solution, we can conclude that it is impossible in this technical solution to reduce the temperature of the exhaust gases to such a level that condensation of water is possible; Known technical solution is absolutely not functional. In the known material there are no signs inherent in the technical solution underlying the present invention and, based on this, the known technical solution is not the technical equivalent of the claimed technical solution according to any of the signs and criteria.

Based on the test results of the prototype of the proposed technical solution integrated into the fuel system of a serial diesel engine, the result is new for the actual practice of operating diesel engines and amounts to $-97 \%$ reduction in the level of soot resulting from the combustion of the fuel mixture in the engine cylinders.

Such a result is completely new for both diesel and gasoline engines, and none of the known technical solutions in any of its signs solves the equivalent technical problem at such a level, which means that the known technical solutions in relation to the proposed technical a solution with proven full performance and documented efficiency, for all four well-known technical solutions with undetermined performance and with unproven efficiency is I technically incompetent. In addition, the prevention of soot formation in engine cylinders due to only hydrodynamic and aerodynamic mixing of standard components of the fuel mixture, which is not known from previous publications and inventions and is also absent in known technical solutions, is in relation to each of the known technical solutions, separately and all opposed technical solutions in the aggregate, completely new.

\section{EXAMPLES OF APPLICATIONS}

[0088] In some embodiments..., - this section provides examples of the various applications of the invention or the various embodiments of the invention, if any.

If a variety of embodiments and applications are not included in the main distinctive features of the claims, it is very important to detail them more clearly and more precisely.

In the future, when identifying and verifying the fact of actual use of the invention, these application samples help to more convincingly prove the facts of use of the invention. 
[0089] In some embodiments..., - this section provides examples of the various applications of the invention or the various embodiments of the invention, if any.

If a variety of embodiments and applications are not included in the main distinctive features of the claims, it is very important to detail them more clearly and more precisely.

In the future, when identifying and verifying the fact of actual use of the invention, these application samples help to more convincingly prove the facts of use of the invention.

[0090] In some embodiments..., - this section indicates thanks to what peculiar features and combinations of features in each of the considered embodiments, the achievement of the ideal final result is provided, which is expected from this particular embodiment.

[0091] In some embodiments..., - this section indicates thanks to what peculiar features and combinations of features in each of the considered embodiments, the achievement of the ideal final result is provided, which is expected from this particular embodiment.

[0092] In some embodiments..., - this section indicates thanks to what peculiar features and combinations of features in each of the considered embodiments, the achievement of the ideal final result is provided, which is expected from this particular embodiment.

[0093] In some embodiments..., - this section indicates thanks to what peculiar features and combinations of features in each of the considered embodiments, the achievement of the ideal final result is provided, which is expected from this particular embodiment.

[0094] In some embodiments..., - this section indicates thanks to what peculiar features and combinations of features in each of the considered embodiments, the achievement of the ideal final result is provided, which is expected from this particular embodiment.

[0095] In some embodiments..., - this section indicates thanks to what peculiar features and combinations of features in each of the considered embodiments, the achievement of the ideal final result is provided, which is expected from this particular embodiment.

[0096] In some embodiments..., - this section indicates thanks to what peculiar features and combinations of features in each of the considered embodiments, the achievement of the ideal final result is provided, which is expected from this particular embodiment.

[0097] In some embodiments..., - this section indicates thanks to what peculiar features and combinations of features in each of the considered embodiments, the achievement of the ideal final result is provided, which is expected from this particular embodiment.

[0098] In some embodiments..., - this section indicates thanks to what peculiar features and combinations of features in each of the considered embodiments, the achievement of the ideal final result is provided, which is expected from this particular embodiment. 
[0099] An operating principle of the devices is..., - this section analyzes and compares the options for operating an apparatus or device, analyzes the options for management and control processes, and application of the principles of forming feedback elements between actuators and control systems or processors.

[0100] This section compares the capabilities and features of the various listed options and examples of application of the invention.

[0101] In some embodiments..., - this section indicates thanks to what peculiar features and combinations of features in each of the considered embodiments, the achievement of the ideal final result is provided, which is expected from this particular embodiment.

[0102] In some embodiments..., - this section indicates thanks to what peculiar features and combinations of features in each of the considered embodiments, the achievement of the ideal final result is provided, which is expected from this particular embodiment.

[0103] It will be appreciated that the..., - this section indicates thanks to what peculiar features and combinations of features in each of the considered embodiments, the achievement of the ideal final result is provided, which is expected from this particular embodiment.

[0104] Various embodiments of the invention allow one or more of the following advantages..., - this section identifies and analyzes any advantages arising from the embodiment of the invention.

[0105] It will be appreciated by those skilled in the art that various omissions, additions and modifications may be made to the methods and structures described above without departing from the scope of the invention. All such modifications and changes are intended to fall within the scope of the invention, as defined by the appended claims.

As an example of an analytical assessment, we give a comparative characteristic of an innovative product, which in general can be represented as, for example, a fuel mixture, is as follows:

○ the product is formed within a short period of time - this time can be equal to at least a few milliseconds, for which the product receives all the necessary characteristics and properties and immediately becomes suitable for further use;

○ the product retains its properties for a long time if the pressure under which it is formed does not change or if it increases;

- the product is able to maintain its properties and characteristics even with a sharp increase in pressure (during testing, the product pressure was up to 2000 bar and no changes in the structure and properties of the product were found);

○ the product has a flexible hydrodynamic structure;

$\circ$ the product has a flexible chemical structure; 
- the product can consist entirely of homogeneous liquids and gases in their properties, while all liquid and gaseous components can be mixed in various proportions and combinations;

○ the product can consist of liquids and gases that are heterogeneous in properties, while all liquid and gaseous components can be mixed in various proportions and combinations;

$\circ$ after formation, various chemicals that are not part of the starting product may be added to the product;

$\circ$ various chemicals that are not part of the starting material may be added to the product during formation.

An invented product can have many varieties. The varieties of the invented product include:

a homogeneous mixture consisting of at least two homogeneous liquids;

- a homogeneous mixture consisting of at least two dissimilar liquids;

○ a mixture consisting of at least two homogeneous liquids and at least one gas;

$\circ$ a mixture consisting of at least two dissimilar liquids and at least one gas;

- a mixture consisting of at least one solution of liquids and at least one additional liquid;

- a mixture consisting of at least two homogeneous solutions of liquids;

- a mixture consisting of at least two heterogeneous solutions of liquids;

- a mixture consisting of at least two homogeneous solutions of liquids and at least one gas;

$\circ$ a mixture consisting of at least two heterogeneous solutions of liquids and at least one gas.

Chemical reagents can be introduced into the mixture both during formation and after formation. These types of mixtures can also have many varieties:

- a mixture consisting of at least two homogeneous liquids with the addition, after formation, of additional chemicals;

- a mixture consisting of at least two dissimilar liquids with the addition of additional chemicals after formation;

- a mixture consisting of at least one liquid solution and at least one liquid with the addition of additional chemicals after formation;

$\circ$ a mixture consisting of at least two homogeneous solutions of liquids with the addition of additional chemical reagents after formation;

- a mixture consisting of at least two heterogeneous solutions of liquids with the addition of additional chemical reagents after formation.

Further it is necessary to reveal the essence of technology, that underlies the formation of a new product - by way of example we give a mixture, which are the production technology is as follows: 
$\circ$ at the moment of formation of the mixture, the level of turbulence at all points of the cross-section of the flows of the basic components of the mixture is equalized, due to which the necessary level of uniformity of state over the entire volume and the required level of particle size of the components of the mixture are achieved;

$\circ$ the kinetic energy of the particles of all components of the mixture is conserved, and this energy is released if the pressure under which the mixture is reduced or even becomes atmospheric;

$\circ$ the release of the kinetic energy of the components of the mixture helps (as, for example, during the combustion process to obtain the maximum atomization of the fuel liquid component of the mixture);

O the number of components in the mixture is practically unlimited, since the apparatus for forming the mixture can be adapted to the required number of components without changing the fundamental principles of the technology;

$\circ$ if a gas or a mixture of gases is used as one or several components, the fundamental principles of the technology make it possible to obtain an aerosol in the form of a mixture or allow mixing the mixture with compressed gas or air;

○ the technology allows sequential introduction of additional components into the mixture, and the number of such components and their chemical composition are not limited;

O since all the parameters of the mixture depend on the pressure in the flow of the components of the mixture, the regulation of the properties and parameters of this mixture can be carried out by adjusting the pressure.

\section{WHAT IS CLAIMED IS...}

1. The first independent claim is the most important for the application:

The first claim should be extremely brief and at the same time consist of three parts:

- The first part gives the commercial name of engineering solution, which should cover the largest possible area of commercial use, and at the same time give an idea of the scope of the claims of the claimed engineering solution, excluding advertising manner of speech and paradoxical statements;

- The second part of the first claim describes the distinctive features, their sets and interrelations, which are known and used as basic for the realization of distinctive features;

- The third part of the first claim describes the distinctive features, their sets and static and dynamic relations of the distinctive features with the basic known features, which together ensure the achievement of an ideal final result. 
Subsequent dependent claims should describe in more detail the distinctive features in the most general form. All subsequent claims should disclose all possible applications of distinctive features to form an effect and achieve an ideal final result. If the ideal final result can be achieved in some options and subject to the availability of different options for combining distinctive features with basic features, all possible options are indicated in the following claims.

Each subsequent claim also consists of three parts, and the requirements for each of them corresponds to the requirements for the first claim.

2. The second independent claim of the invention.

3. The claim dependent on the first and third claims of the invention.

4. The claim dependent on the first and third claims of the invention.

5. The dependent on the first and third claims of the invention.

6. The dependent on the first and third claims of the invention.

7. The third independent claim of the invention.

If the subject of the invention, - a device or apparatus and the named, - is an apparatus, for example, it is preferable to have at least three independent claims, so that the first claim is, - apparatus for..., method of application and associated method of manufacture.

The third claim, - the method of the apparatus application.

8. The eighth claim - the apparatus for... and associated method of manufacture.

All of the above is just an example, each engineering solution has its own peculiar features, and, accordingly, the composition and structure of the construction of the claims may change.

\section{ABSTRACT OF THE DISCLOSURE}

Abstract, in principle, is not special and there are no recommendations for its composition.

\section{LIST OF REFERENCES, PATENT AND LICENSE MATERIALS}

\section{ANNEX 1}




\title{
ARTIFICIAL INTELLIGENCE DATA PROCESSING SYSTEM AND METHOD
}

\begin{abstract}
There are provided a system that is capable of performing tasks associated with IPR procurement. The system employs a computing architecture that provides characteristics of artificial intelligence (AI). The computing architecture employs a configuration of pseudoanalog variable-state machines that is implemented by disposing the pseudo-analog variablestate machines in a hierarchical arrangement, wherein pseudo-analog variable-state machines higher in the hierarchical arrangement mimic behavior of a human claustrum for performing higher cognitive functions when processing information associated with one or more service requests and for performing quality checking of the one or more work products. Moreover, the computing architecture is susceptible to being implemented by employing a novel configuration of data processing devices, for example arrays of RISC processors.
\end{abstract}

\section{ANNEX 2}

United States Patent Application

20190205606

Kind Code

Zhou; Shaohua Kevin ; et al.

July 4, 2019

\section{METHOD AND SYSTEM FOR ARTIFICIAL INTELLIGENCE BASED MEDICAL IMAGE SEGMENTATION}

\begin{abstract}
Methods and systems for artificial intelligence based medical image segmentation are disclosed. In a method for autonomous artificial intelligence based medical image segmentation, a medical image of a patient is received. A current segmentation context is automatically determined based on the medical image and at least one segmentation algorithm is automatically selected from a plurality of segmentation algorithms based on the current
\end{abstract}


segmentation context. A target anatomical structure is segmented in the medical image using the selected at least one segmentation algorithm.

\title{
ANNEX 3
}

United States Patent Application

20190206218

Kind Code

KUSENS; BRUCE HOWARD ; et al.

July 4, 2019

\section{UTILIZING ARTIFICIAL INTELLIGENCE TO DETECT OBJECTS OR PATIENT SAFETY EVENTS IN A PATIENT ROOM}

\begin{abstract}
Methods and systems are provided for detecting objects or patient safety events in a patient room. Artificial intelligence is utilized to enhance safety issue recognition capabilities by the methods and systems. Sensors collect a series of images and depth data in a room of a patient. Data (corresponding to images and depth data of an object or patent safety event) is received from the sensors and compared to stored data to identify the object or patient safety event. The images are communicated to a central video monitoring system and a user may be prompted to confirm if the object requires learning or a patient safety event occurred (or identify the object or patient safety event) or to provide additional parameters or actions. A patient safety learning system analyzes the data and incorporates the user response to enhance safety issue recognition capabilities of the system and reduce false alerts.
\end{abstract}

\section{ANNEX 4}

United States Patent Application

20190189236

Kind Code

A1

Poliakov; Pavel ; et al.

June 20, 2019

\section{ARTIFICIAL INTELLIGENCE BASED MONITORING OF SOLID STATE DRIVES AND DUAL IN-LINE MEMORY MODULES}




\begin{abstract}
In embodiments, a memory controller (MC) includes an output interface, and an execution engine (EE) to identify, based on field test results of a die coupled to the $\mathrm{MC}$, initial test results of the die using an artificial neural network (ANN) trained to identify the die from a set of NVM dies based on initial test results of the set of NVM dies obtained at a time of manufacture of the set of dies. The initial test results include a first useful life prediction and the field test results include a second useful life prediction, and the initial test results are regenerated by the ANN to protect their confidentiality. In embodiments, the MC is further to compare the second useful life prediction with the first useful life prediction, to determine a deviation between the two, and output, via the output interface, the deviation to a user.
\end{abstract}

\title{
ANNEX 5
}

United States Patent Application

20190205885

Kind Code

A1

Lim; Chern Jie ; et al.

July 4, 2019

\section{MACHINE LEARNING ENGINE FOR FRAUD DETECTION FOLLOWING LINK SELECTION}

\begin{abstract}
A machine learning engine for fraud detection following link selection may be trained using artificial intelligence techniques and used according to techniques discussed herein. A buyer account may be used to establish and generate a digital gift card having a particular value specified by the buyer. The digital gift card may then be conveyed to another account, such as an email address. The digital gift card may be provided with an online electronic process for redemption and use of the value, for example, by selecting a link and navigating to the process. When the claimer account attempts to utilize the value of the gift card by navigating to the process or otherwise engaging in the electronic process through a device, a risk and fraud analysis engine may execute to determine, based on real-time data of the claimer account, the buyer account, and/or device, whether the digital gift card was generated fraudulently or is being used fraudulently.
\end{abstract}

\section{ANNEX 6}




\title{
DEVICE OF CONTROLLING RELATED DEVICE USING ARTIFICIAL INTELLIGENCE BASED ON OPERATION SITUATION, SCHEDULE BOT AND SERVER CONTROLLING THEREOF
}

\begin{abstract}
This application is a technology with regard to a device that controls a related device based on an operation situation using artificial intelligence, a schedule bot and a server that controls the same, and the device that receives a control of the related device based on the operation situation by an exemplary embodiment of this application includes a function unit that performs a physical function of the device, a communication unit that receives a time schedule and function information from a related device or a schedule management device, and control unit that controls the function unit to maintain a ready state so that a function unit performs a first function at a point that is indicated in the time schedule by using the time schedule and the function information that the communication unit receives.
\end{abstract}

\section{ANNEX 7}

United States Patent Application

20190197357

Kind Code

A1

ANDERSON; GLEN J. ; et al.

June 27, 2019

\section{MISUSE INDEX FOR EXPLAINABLE ARTIFICIAL INTELLIGENCE IN COMPUTING ENVIRONMENTS}

\begin{abstract}
A mechanism is described for facilitating misuse index for explainable artificial intelligence in computing environments, according to one embodiment. A method of embodiments, as described herein, includes mapping training data with inference uses in a machine learning environment, where the training data is used for training a machine learning model. The
\end{abstract}


method may further include detecting, based on one or more policy/parameter thresholds, one or more discrepancies between the training data and the inference uses, classifying the one or more discrepancies as one or more misuses, and creating a misuse index listing the one or more misuses.

\title{
ANNEX 8
}

United States Patent Application

20190187775

Kind Code

Rotem; Nadav ; et al.

June 20, 2019

\section{DYNAMIC POWER MANAGEMENT FOR ARTIFICIAL INTELLIGENCE HARDWARE ACCELERATORS}

\begin{abstract}
A computer-implemented method for dynamically managing the power usage and/or performance of an artificial intelligence (AI) hardware accelerator may include (1) receiving an instruction stream that includes one or more instructions for performing at least one AIspecific computing task, (2) identifying a plurality of special-purpose, hardware-based functional units configured to perform AI-specific computing tasks, (3) predicting, based on an analysis of at least a portion of the instruction stream, a power-usage requirement for at least one of the functional units when executing the instruction stream, and then (4) modifying, based on the power-usage requirement, the power supplied to at least one of the functional units. Various other methods and systems are also disclosed.
\end{abstract}

\section{ANNEX 9}

United States Patent Application

20190188570

Kind Code

June 20, 2019 


\title{
METHODS AND APPARATUS FOR MODEL PARALLELISM IN ARTIFICIAL NEURAL NETWORKS
}

\begin{abstract}
The method according to an embodiment comprises automatically controlling allocation, to memories of available hardware resources, of parameters defining computational operations required to calculate an output of at least one layer of neurons of an artificial neural network. The allocation is controlled on the basis of previously-defined allocation data specifying how the operations required to calculate the output of the one layer of neurons are to be allocated to hardware resources to perform the operations. The allocation data is pre-defined using, at least partly, an automatic computer-implemented process, which may include checking before each iteration of the network which of the hardware resources are available to execute that iteration of the network and, if necessary, re-defining the allocation data for that iteration accordingly
\end{abstract}

\section{ANNEX 10}

United States Patent Application

20190192258

Kind Code

A1

KANG; Je Hun ; et al.

June 27, 2019

\section{AUTOMATIC STEPWISE TOOTH MOVEMENT SYSTEM AND METHOD USING ARTIFICIAL INTELLIGENCE TECHNOLOGY}

\begin{abstract}
Disclosed is a stepwise automatic orthodontic system and method using an artificial intelligence technique. The method includes: scanning a dental state of a patient by using an intraoral scanner; allowing a server to determine to which group of grouped data of the database the scanned dental data belong; allowing the server to refer to data of the determined group, move a tooth needing orthodontics gradually, and generate a predictive digital orthodontic dental data set; allowing the server to transmit the orthodontic-processed digital orthodontic dental data set of a patient to a 3D printer, and allowing the 3D printer to generate and output a dental orthodontic model; and generating a clear aligner by vacuum-compressing a transparent synthetic resin plate to the generated dental orthodontic model through a vacuum former. In the exemplary embodiment of the present invention, the orthodontic patient is clustered or grouped through an unsupervised learning based on the good orthodontic data
\end{abstract}


excluding personal information of the patient, and the tooth moving plan for orthodontics through repeated reinforcement learning satisfying the orthodontic limit condition suggested by the grouped data and the orthodontics textbook for respective steps.

\title{
ANNEX 11
}

\section{METHOD AND SYSTEM FOR CLASSIFYING AN OBJECT-OF-INTEREST USING AN ARTIFICIAL NEURAL NETWORK}

\begin{abstract}
Methods, systems, and techniques for classifying an object-of-interest using an artificial neural network, such as a convolutional neural network. An artificial neural network receives a sample image including the object-of-interest overlaying a background and a sample background image excluding the object-of-interest and corresponding to the background overlaid by the object-of-interest. The object-of-interest is classified using the artificial neural network. The artificial neural network classifies the object-of-interest using the sample background and sample images. Prior to receiving the sample background and sample images the artificial neural network has been trained to classify the object-of-interest using training image pairs. Each of at least some of the training image pairs includes a first training image that includes a training object-of-interest overlaying a training background and a training background image excluding the training object-of-interest and corresponding to the training background.
\end{abstract}

\section{ANNEX 12}

United States Patent Application

20190171950

Kind Code

Srivastava; Kumar

June 6, 2019 
METHOD AND SYSTEM FOR AUTO LEARNING, ARTIFICIAL INTELLIGENCE (AI) APPLICATIONS DEVELOPMENT, OPERATIONALIZATION AND EXECUTION

\begin{abstract}
Disclosed is a method and a system a method and a system for auto learning, artificial intelligence (AI) applications development, and execution. Various applications or operations may be associated with training environment-agnostic AI models, automated AI app application performance monitoring, fault, quality and performance remediation through prediction of failures or suboptimal performance, privacy and secure AI training and inference mechanism for data and AI model sharing between untrusted parties, and building auto learning applications that can automatically learn and improve.
\end{abstract}

\title{
ANNEX 13
}

United States Patent Application

Kind Code

LU; Mingzhu
20190171944

A1

June 6, 2019

\section{INTEGRITY EVALUATION OF UNSTRUCTURED PROCESSES USING ARTIFICIAL INTELLIGENCE (AI) TECHNIQUES}

\begin{abstract}
A process integrity evaluation system ensures integrity of unstructured processes. The process integrity evaluation system handles structured, semi-structured, and unstructured data at massive and large scale. The system provides scalability, secure storage, indexing, knowledge storage, and visualizations of processes by information retrieval, natural language processing, cloud computing, large scale machine learning, knowledge discovery, and other artificial intelligence techniques. Self-provided data, systematically gathered data, and potentially related data from additional sources are incorporated in the process integrity evaluation system which provides the core capabilities of data integrity checking, entity extraction, entity resolution, entity categorization, entity relationship extraction, processes extraction and reconstruction based on knowledge storage, such as knowledge graphs, inference functions, and evaluation computations. After extracting and reconstructing unstructured processes successfully, machine learning functions compute an integrity assurance score, e.g., a similarity, between extracted documents and the internal records in addition to an evaluation result, which can ensure the integrity of the unstructured processes.
\end{abstract}




\title{
ANNEX 14
}

United States Patent Application

20190140913

Kind Code

A1

GUIM BERNAT; Francesc ; et al.

May 9, 2019

TECHNIQUES FOR ARTIFICIAL INTELLIGENCE CAPABILITIES AT A NETWORK SWITCH

\begin{abstract}
Examples include techniques for artificial intelligence (AI) capabilities at a network switch. These examples include receiving a request to register a neural network for loading to an inference resource located at the network switch and loading the neural network based on information included in the request to support an AI service to be provided by users requesting the AI service.
\end{abstract}

\section{ANNEX 15}

United States Patent Application

20190171928

Kind Code

A1

Young; Robin

June 6, 2019

\section{DYNAMICALLY MANAGING ARTIFICIAL NEURAL NETWORKS}

\begin{abstract}
In some embodiments, the disclosed subject matter involves using socket layers with a plurality of artificial neural networks in a machine learning system to create customizable inputs and outputs for a machine learning service. The machine learning service may include a plurality of convolutional neural networks and a plurality of pre-trained fully connected neural networks to find the best fits. In an embodiment, when the customized input or output data is not a good fit with the pre-trained artificial neural networks, a socket layer may automatically request
\end{abstract}


additional convolutional layers or new training of a neural network to dynamically manage the machine learning system to accommodate the customized input or customized output. Other embodiments are described and claimed.

\title{
ANNEX 16
}

\section{SEARCH RESULT AGGREGATION METHOD AND APPARATUS BASED ON ARTIFICIAL INTELLIGENCE AND SEARCH ENGINE}

\begin{abstract}
The present disclosure provides a search result aggregation method and apparatus based on artificial intelligence and a search engine. The method includes: obtaining a query; generating a plurality of search results according to the query; obtaining a plurality of corresponding demand dimensions according to the query; aggregating the plurality of demand dimensions according to the plurality of search results; obtaining an answer corresponding to each demand dimension, and aggregating the answers corresponding to the plurality of demand dimensions according to the aggregated demand dimensions to generate an aggregation result.
\end{abstract}

\section{ANNEX 17}

United States Patent Application

Kind Code

Emma; Maria ; et al.
20190156222

A1

May 23, 2019 


\begin{abstract}
System, methods, and apparatuses for an artificial intelligence (AI) toy with improved conversational dialogue and personality development. The AI toy determines responses to stimuli based on user profiles and personality profiles that are developed through user interaction and external media inputs. Natural Language Processing (NLP) and other semantic interaction processing is paired with the profiles to develop AI personality and conversational ability.
\end{abstract}


DOI 10.32370/IA_2020_01_6

\title{
Development of a Patenting and Licensing Strategy - principles of development of control and analytical algorithms taking into consideration new opportunities provided by quantum computers in light of analysis of readiness of a smart technology and its smart infrastructure for mass production and efficient integrated marketing
}

\author{
Yerzhan Kusparmakov \\ - Engineer, Inventor, Doctor of Philosophy (PhD) in Computer Science \\ - Professional Member of the New York Academy of Science \\ - Academician of the International Informatization Academy \\ - Advisor of the Russian Academy of Natural History \\ - Chief Product Manager at SAMSUNG Electronics
}

e-mail:kusparmakov@gmail.com

\begin{abstract}
Recently several new, in many respects - completely new areas of development of high-tech innovative products, which are unambiguously mass-market products, have emerged.

Such innovative areas may include the said and classified as - smart products and technologies, such products as - smart design, smart medical technologies, equipment and tool set, definitely including medical equipment and devices with efficient mobile applications, smart transport and smart transportation, smart manufacturing and smart manufacturing equipment, including special processing equipment with digital programmed control and the most perfect machining centers and flexible production modules.
\end{abstract}

Keywords: Patenting, Licensing, Quantum computers, Smart technology, Smart infrastructure, Mass production Recently several new, in many respects - completely new areas of development of high-tech innovative products, which are unambiguously mass-market products, have emerged.

Such innovative areas may include the said and classified as - smart products and technologies, such products as - smart design, smart medical technologies, equipment and tool set, definitely including medical equipment and devices with efficient mobile applications, smart transport and smart transportation, smart manufacturing and smart manufacturing equipment, including special processing equipment with digital programmed control and the most perfect machining centers and flexible production modules.

It also includes Smart workforce development.

Let us consider this whole process on the example of introduction of modifications of mobile secure coding of digital external storage media. 


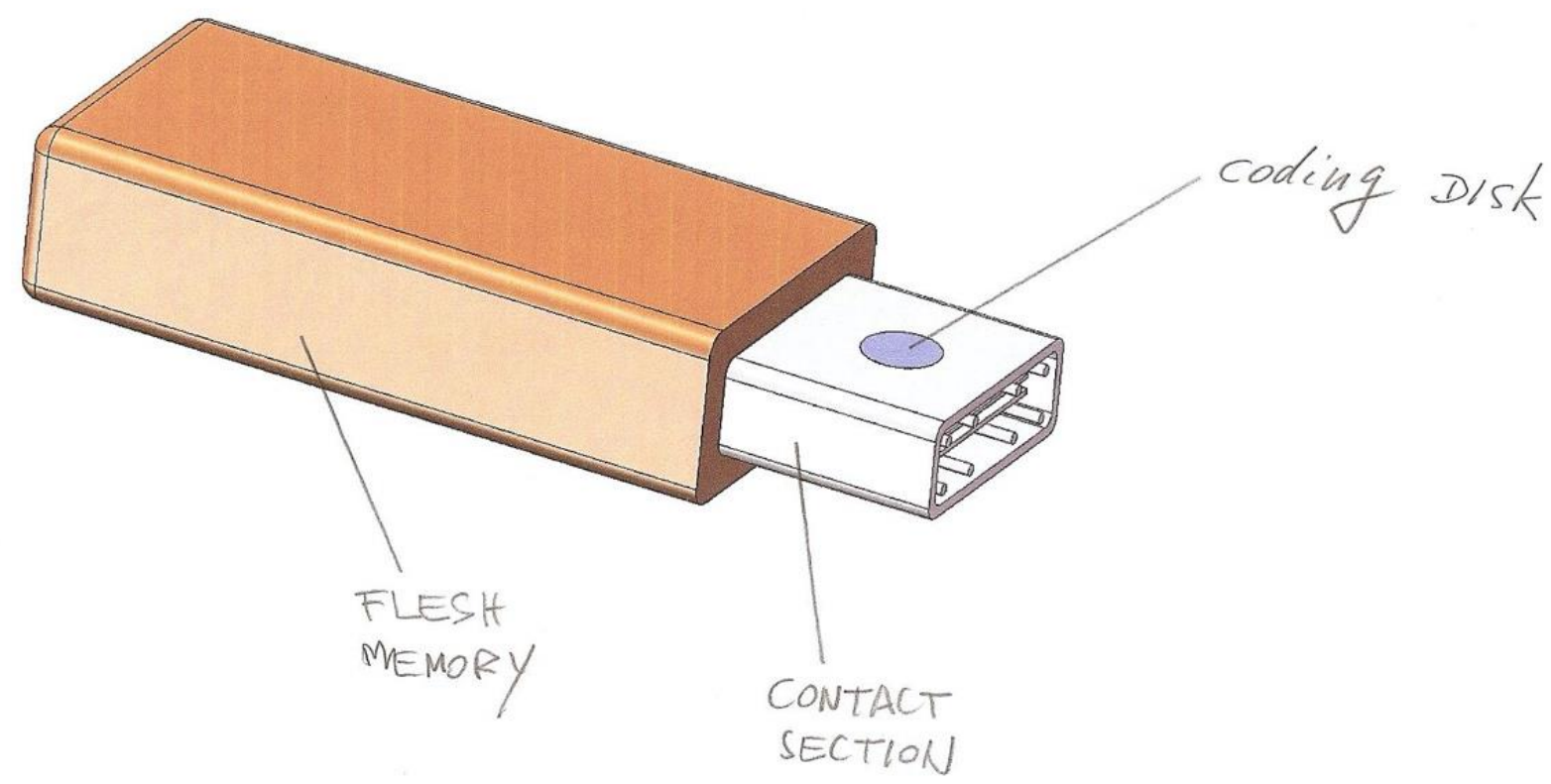

Figure 1, - an example of such an external storage medium modified by the innovative coding system is presented in the drawing

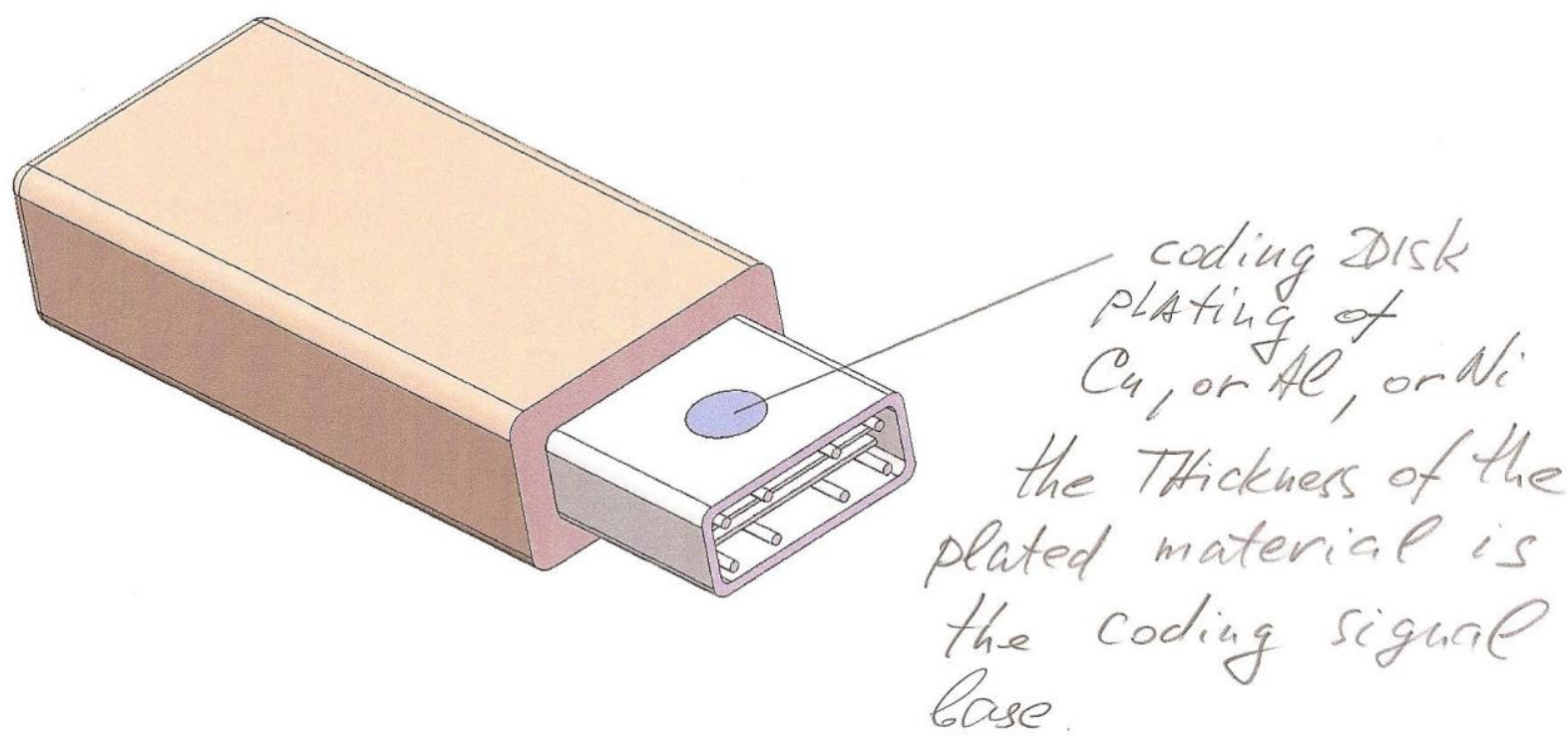

Figure 2, - an example of such an external storage medium modified by the innovative coding system is presented in the drawing as well

The principle of the coding system and materials used for its construction and their combinations are also shown in the drawing. 
A solution of such a coding and decoding unit, with all its simplicity, allows obtaining maximum accuracy of code identification, since the specified accuracy is determined by the accuracy of a thickness of a coding or decoding disk.

There are at least two methods of manufacturing and installation of this disk in a casing, - the first method is the method of manufacturing of a coding disk of precision foil, the second method is applying of a precision electrochemical coating in a groove made in the casing for the coding disk.

\section{General information}

All projects of this group of technical solutions are based on one method of coding and subsequent identification of record of a coding element.

The essence of the principle is in applying a coding coating or its technological equivalent on a secured object and further measuring the thickness of this coating, which determines coincidence or non-coincidence of measuring results with the code.

In case of coincidence of an obtained result with the set one there is positive identification of the coding element, in case of non-coincidence, - there is negative identification and stop or blocking of an operating cycle of equipment or information consumer, for example, - a computer. 


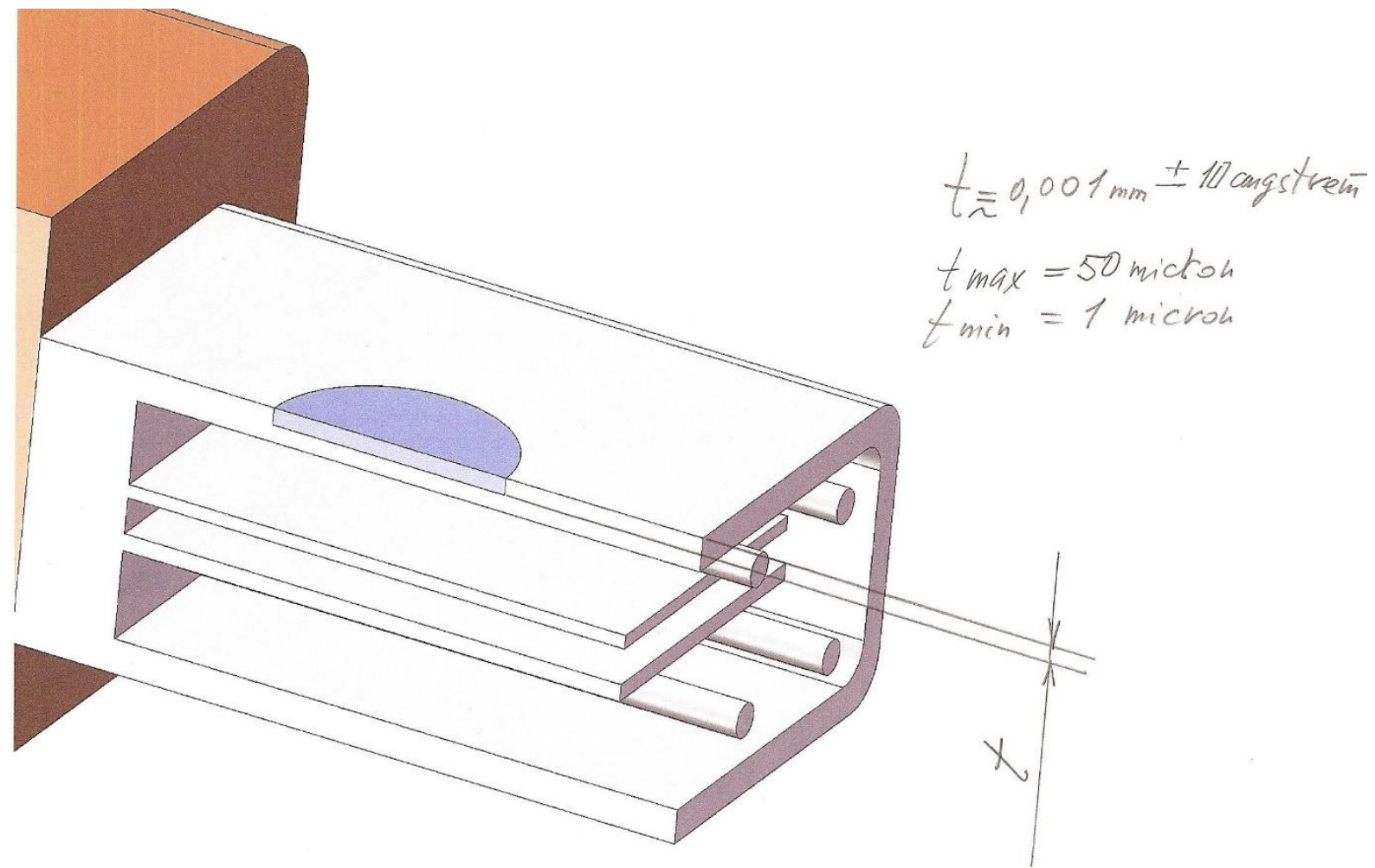

Figure 3, - an example of such an external storage medium with indication of technical details modified by the innovative coding system is also presented in the drawing

The most critical value determining the accuracy of coding or decoding is shown in the section, - the thickness of the disk - $t$.

The minimal thickness may be equal to 1 micron, the maximum thickness -50 microns.

\section{Additional technological characteristics}

Technologically matters of applying special coatings are solved, and this technology was checked many times on analogous tasks related to control of film thickness on solar arrays and in traditional semiconductor manufacturing.

Additional characteristics and possibilities of technology use, with regard to new conditions that emerged on the market of storage media and information tanks for the last year taking into account emerged new courses in technical solutions and technical systems of any levels eventually leading to synthesis of the so-called smart technologies and complex technical systems with elements of artificial intelligence and artificial neural networks. 
Patenting \& Licensing Strategy

$\underline{1}^{\text {st }}$ INITIAL STAGE OF PROJECT - NEW INTERNET TECHNOLOGY PRINCIPLES (RESEARCH)

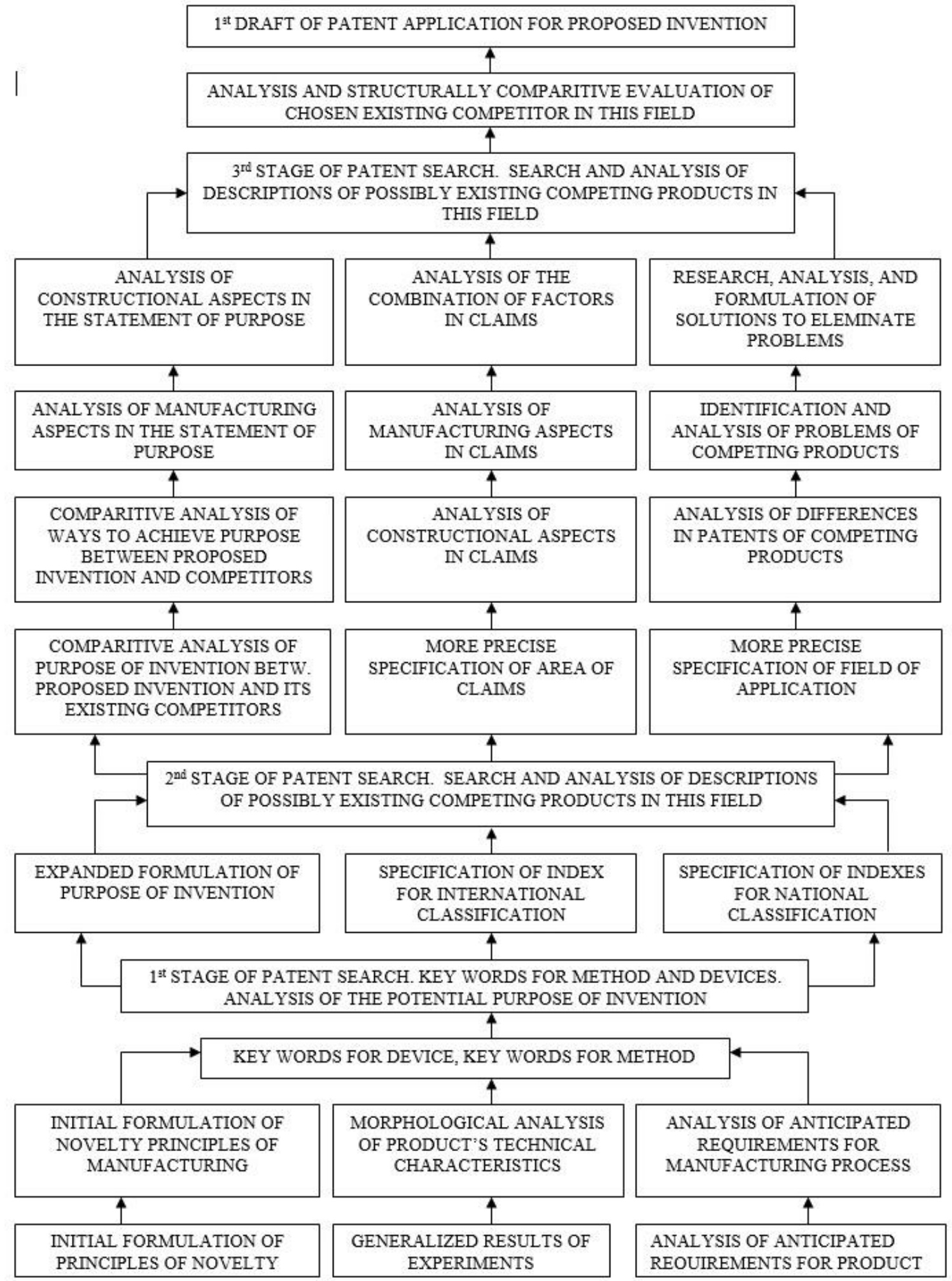

Figure 4, - an algorithm of using the patenting and licensing strategy at initial stages of project development is presented in the drawing 
Patenting \& Licensing Strategy

$2^{\text {nd }}$ INITIAL STAGE OF PROJECT - Codification Technology Principles and Internet integrated solutions DEALS WITH CORE TECHNOLOY THAT RELATES TO BASE TECHNOLOGY AND CONSTRUCTION PRINCIPLES OF PRODUCT ("BASE"), AS OPPOSED TO A SPECIFIC APPLICATION OF THE PRODUCT

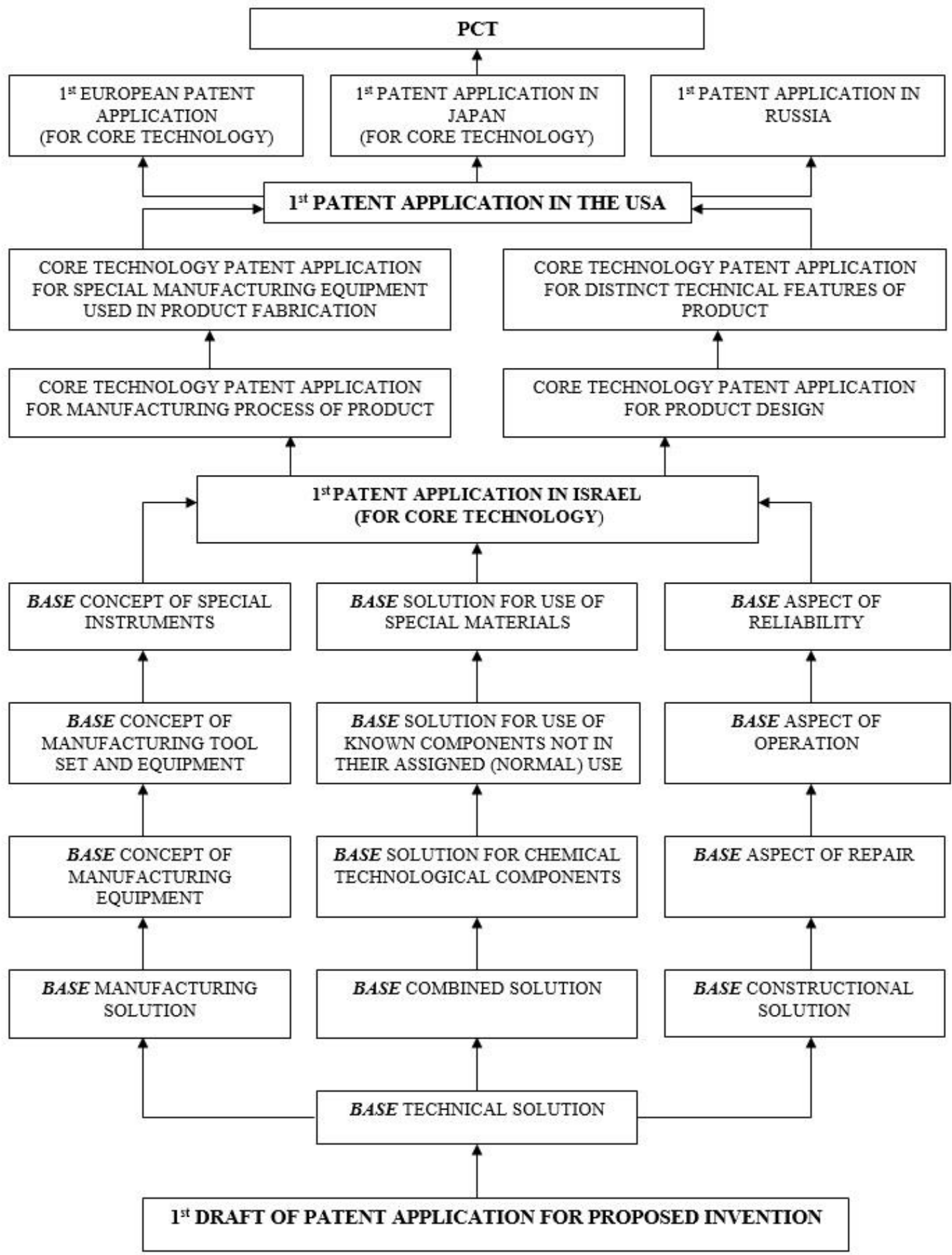

Figure 5, - an algorithm of using the patenting and licensing strategy at subsequent stages of project development is also presented in the drawing 
Patenting \& Licensing Strategy

Codification Technology Principles, Coder-Encoder system and Internet solutions and interfaces $3^{\text {rd }}$ INITIAL STAGE OF PROJECT (RELATING TO SPECIFIC APPLICATION)

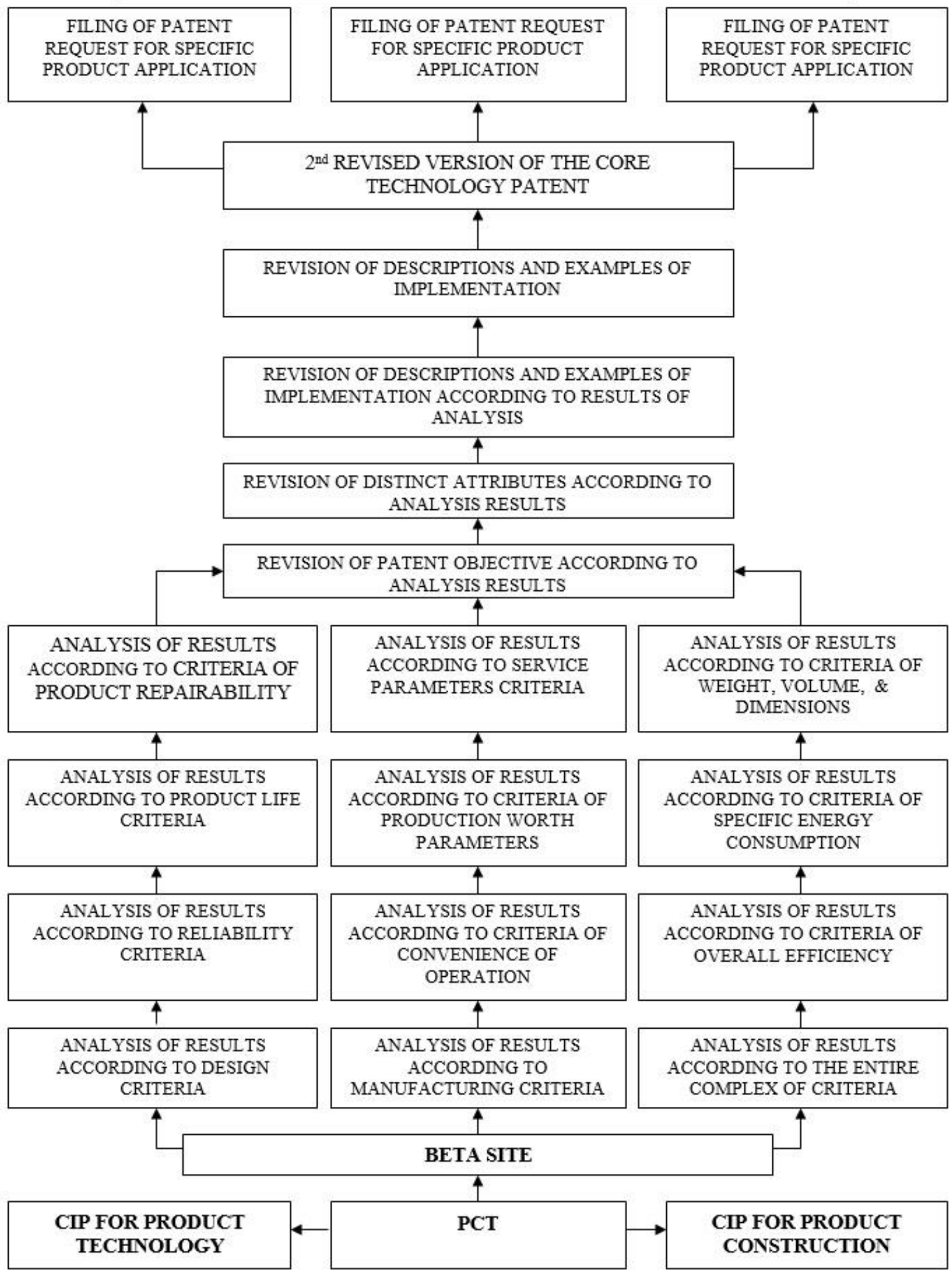

Figure 6, - an algorithm of using the patenting and licensing strategy at medium stages of project development is presented in the drawing 
Patenting \& Licensing Strategy

STAGES: DETAIL DESIGN FOR MASS PRODUCTION of CODIFICATION TECHNOLOGY PRINCIPLES and CODER-ENCODER systems FOR Internet solutions, - COMPONENTS PRELIMINARY TESTING DOCUMENTATION FOR MASS PRODUCTION

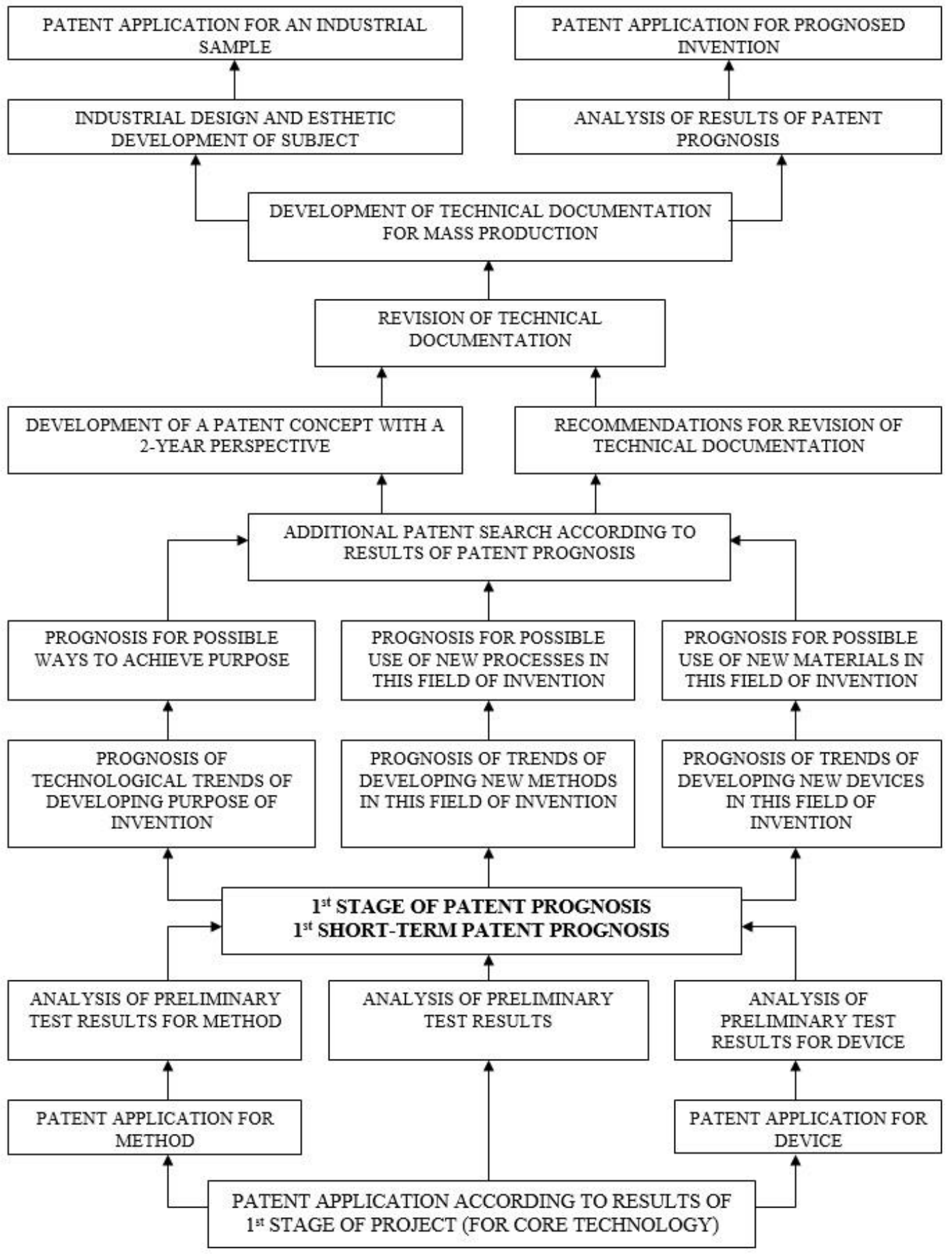

Figure 7, - an algorithm of using the patenting and licensing strategy at design stages for mass production is presented in the drawing 
Patenting \& Licensing Strategy

Codification Technology Principles, Coder-encoder system and Internet new solutions and interfaces STAGES:
TRIAL RUN OF MASS PRODUCTION
PRODUCT ACCEPTANCE TESTING
FULL SCALE MASS PRODUCTION

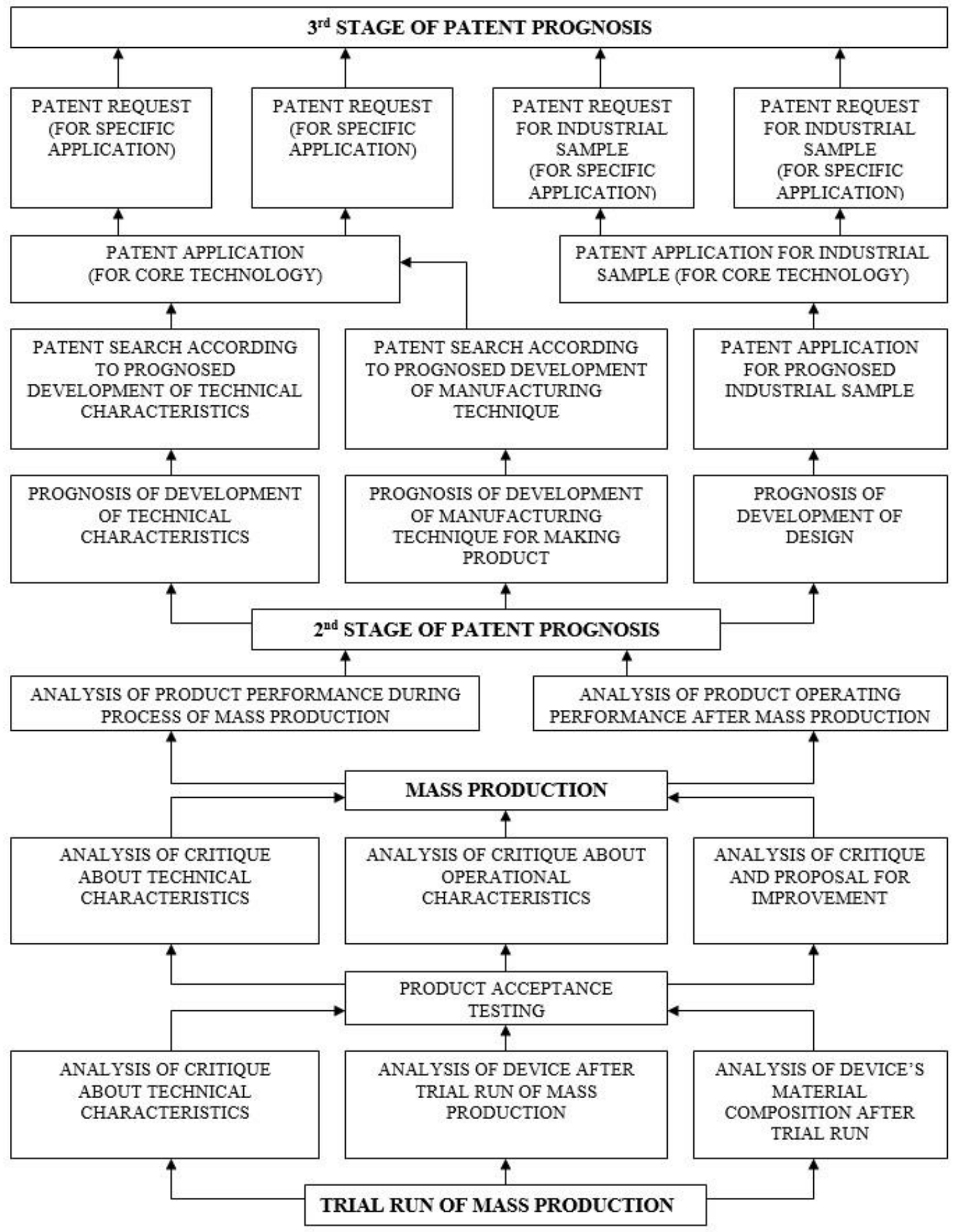

Figure 8, - an algorithm of using the patenting and licensing strategy at stages of mass production, stages of acceptance and qualification tests and after adjustment based on their results transition to full-scale mass production is presented in the drawing 
Patenting \& Licensing Strategy

CODIFICATION TECHNOLOGY PRINCIPLES in Internet solutions, programs and interfaces STAGES:

MASS PRODUCTION

ACTIVE MARKETING PHASE

PRODUCT IMPROVEMENT

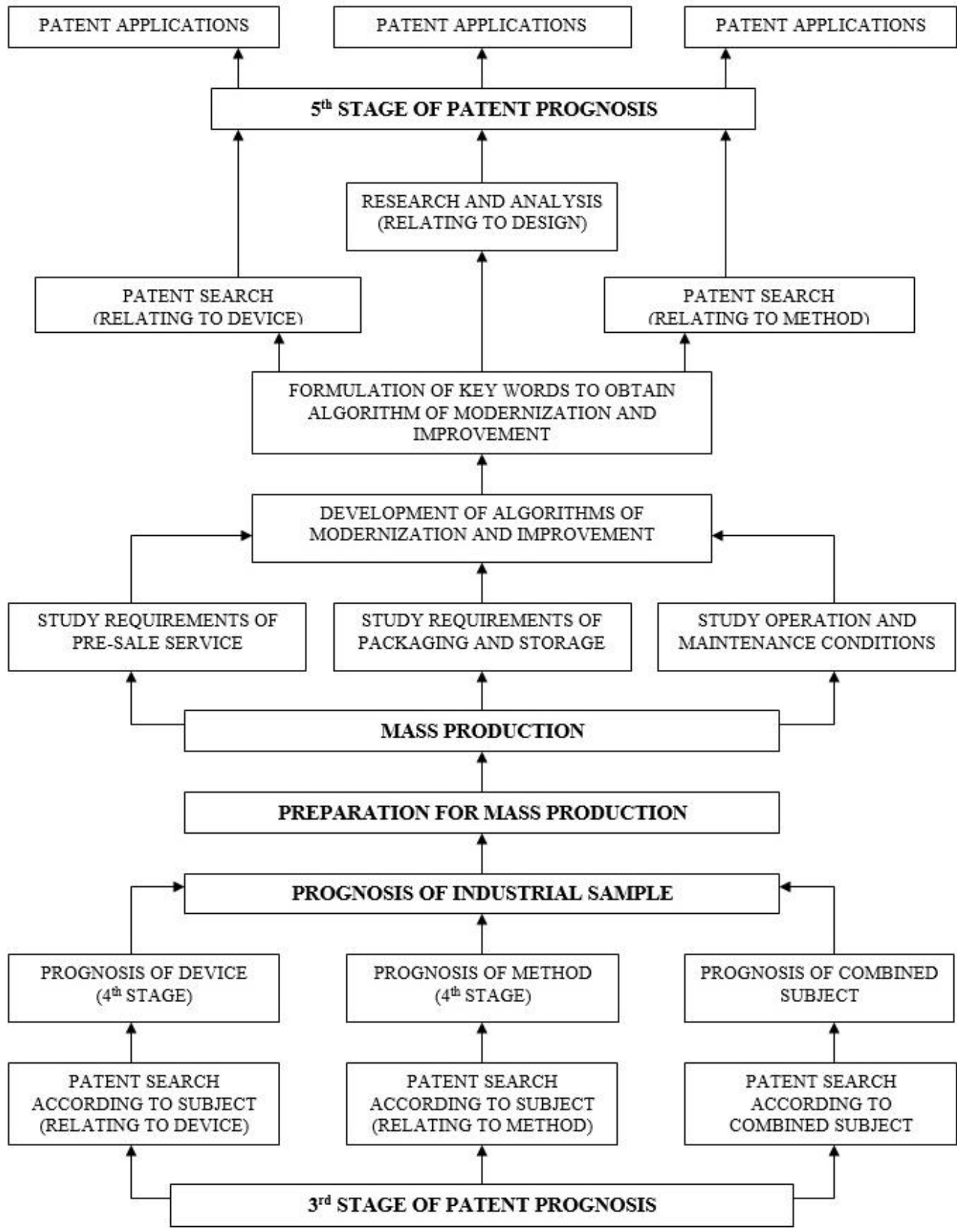

Figure 9, - an algorithm of development and introduction of the patenting and licensing strategy at the stages of project development is presented in the drawing, - such as, - mass production, an active phase of complete marketing and subsequent after them and parallel to them an integrative stage of optimization and modification of the product imparting the product properties and characteristics, - of a smart product manufactured by a smart 
technology, on smart equipment, using smart materials and components, using smart workforce within the framework of the smart production infrastructure

A special attention (in accordance with the algorithm) is paid to patent forecasting in terms of the device, in terms of - methods, in terms of - the combined and integrated object, to the extent practicable using elements of artificial intelligence with artificial neural networks in all component parts of the product infrastructure.

From the marketing experience after implementation of all aspects and features of mass production of a new product, analytical requirements are introduced to the algorithm to all elements of the marketing process service system, - study of pre-sale service, study of packaging and storage requirements, as well as study of requirements and conditions (especially technical requirements and technical conditions) to a procedure and methodology of repair and maintenance.

All these types of activity provide for an integrative combination of all above mentioned aspects and based on them possible objective and task setting on optimization and further project development.

\section{Comments to algorithms (shown on the drawings above)}

Figure 4, - an algorithm of using the patenting and licensing strategy at initial stages of project development is presented in the drawing.

Specific issues solved on this fragment of the algorithm:

- formulation of general novelty of the product;

$\circ$ formulation of general operating performance of the product;

○ formulation of a technical specification of the product;

- formulation of a manufacturing specification of the product;

○ formulation of basic technical requirements to the product and its production;

○ formulation of patent search requirements;

- formulation of technical characteristics of the product and its potential of comparative novelty and potential for the next 5, 10, 15 years.

Figure 5, - an algorithm of using the patenting and licensing strategy at subsequent stages of project development is also presented in the drawing.

Beginning of the algorithm - development of the first option of patent application on the new product with due account for all characteristics and results of all types of patent search, as well as market researches.

Completion of the algorithm - development and filing an international patent application with due account for all results of market researches and its requirements to the new product. 
A nature of basic conceptual solutions on all characteristics and properties of the new product are constantly analyzed in perfection of the algorithm.

Figure 6, - an algorithm of using the patenting and licensing strategy at medium stages of project development is presented in the drawing.

Beginning of the algorithm - development and filing an international patent application with due account for all results of market researches and its requirements to the new product, as well as development of the new product project and its applications in the format - program, system and associated method on the method - CIP, - continuation in part.

System analysis of results of pilot production and all types of tests.

Completion of the algorithm - preparation and filing system applications for supposed inventions, on materials of pilot production, on results of tests and on results of system analysis of all obtained results of this stage of production and implementation of the new product project.

Figure 7, - an algorithm of using the patenting and licensing strategy at design stages for mass production is presented in the drawing.

Beginning of the algorithm - preparation and filing system applications for supposed inventions, on materials of pilot production, on results of tests and on results of system analysis of all obtained results of this stage of production and implementation of the new product project.

Completion of the algorithm - preparation of materials and filing an application for obtaining an industrial sample for the new product.

Apart from that, this algorithm included all phases and stages of development of technical documentation, starting from stages of terms of reference, technical proposal, draft design, technical project, detailed design, corrections of documentation based on results of tests and pilot production.

Also, this algorithm included stages of patent search on arrays of world information with due account for results of tests and their structural analysis.

Figure 8, - an algorithm of using the patenting and licensing strategy at stages of mass production, stages of acceptance and qualification tests and after adjustment based on their results transition to full-scale mass production is presented in the drawing.

Beginning of the algorithm - mass production of the new product

Completion of the algorithm - 3 stages of patent forecasting, for detecting courses of optimization of the new product. 


\section{Theory of Inventive Problem Solving (TIPS) and Algorithms of Inventive Problem Solving (AIPS)}

Theory of Inventive Problem Solving and all known its derivative Algorithms of Inventive Problem Solving were created in the USSR and in the period from 1945 to 1947, where special emphasis was not laid on commercialization of innovative solutions, one may even say that at a certain stage of creation of the Theory and Algorithm of Inventive Problem Solving the issues of commercialization were ignored deliberately in favor of purely technological options of innovative solutions, absolutely detached from the real economy and in majority cases from the real life.

As the result of such short-sighted and one-sided organizational model of development of the innovative process, which took place at that time, inventors, who grew up and were brought up on classical TIPS and AIPS approaches and principles turned out completely unprepared for peculiarities and methods of competitive fight under the conditions of the modern society with free competitive economy, especially with gradual transition to system smart technologies, especially using elements of artificial intelligence and artificial neural networks.

They are willing to and like to invent, but are not prepared for and are not able to earn on their inventions, in order to get worthy compensation for their talent and creative work.

It is especially important to evaluate the necessity of beginning of the innovative process and understand, and even better to estimate all possible options of sequence of events in the commercialization process.

For this purpose, we find it expedient to use a system of assessment algorithmic criteria and tables represented by analytical assessment sheets, the algorithm of patenting and licensing strategy consisting of 6 group elements of assessment of criteria and parameters of an emerged innovative idea or initiative and process of their development and implementation.

Due to emergence of new formats of recording and reading on optical storage media using blue lases, and due to beginning of production of multilayer optical disks, based on the same technology, the proposed principles and technical solutions on secure coding have acquired even more value, since the quantity of recorded information on each disk increases and absence of protection leads to more loses of secret and confidential data.

In addition to already transferred information it is necessary to specify possibilities on coding each layer in multilayer disks, in which each level of record layers is coded, which is a substantial improvement of the formatting system of the optical storage medium in threedimensional expression and is a tool of ensuring (for especially important and secret information) local selective coding of information within one disk. 


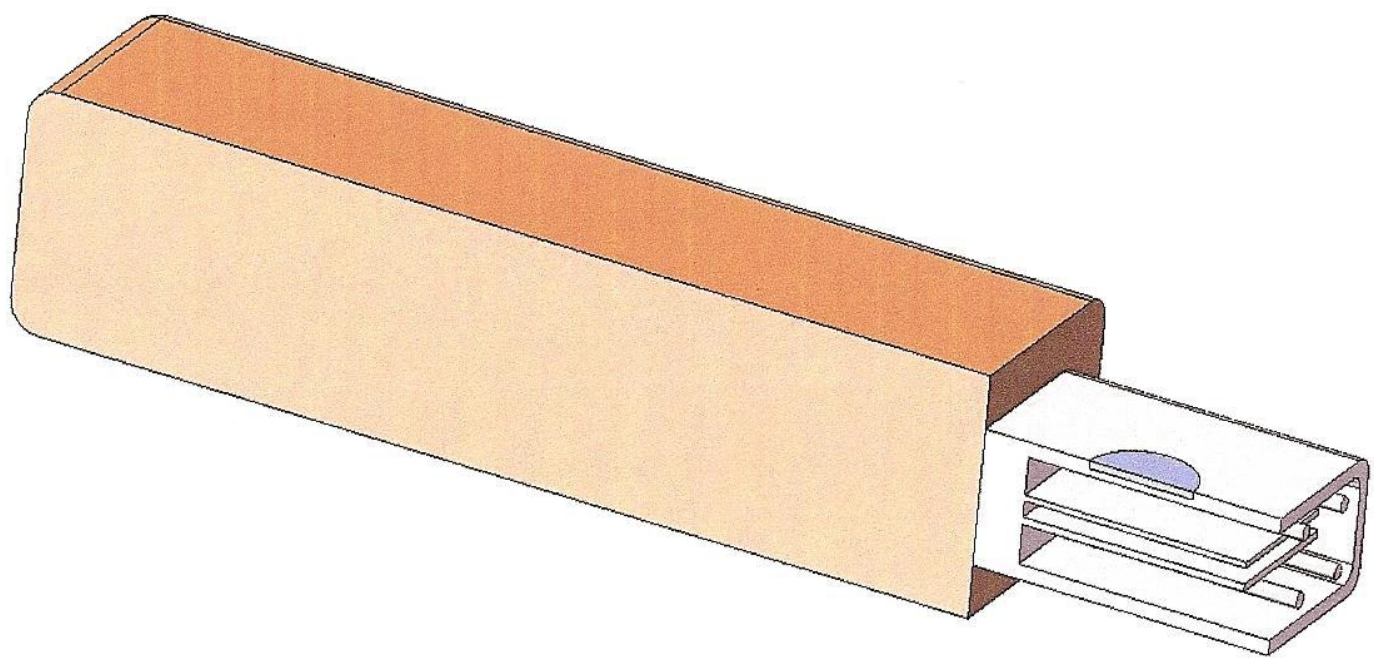

Figure 10, - a model of a storage medium in axial section is shown in the drawing

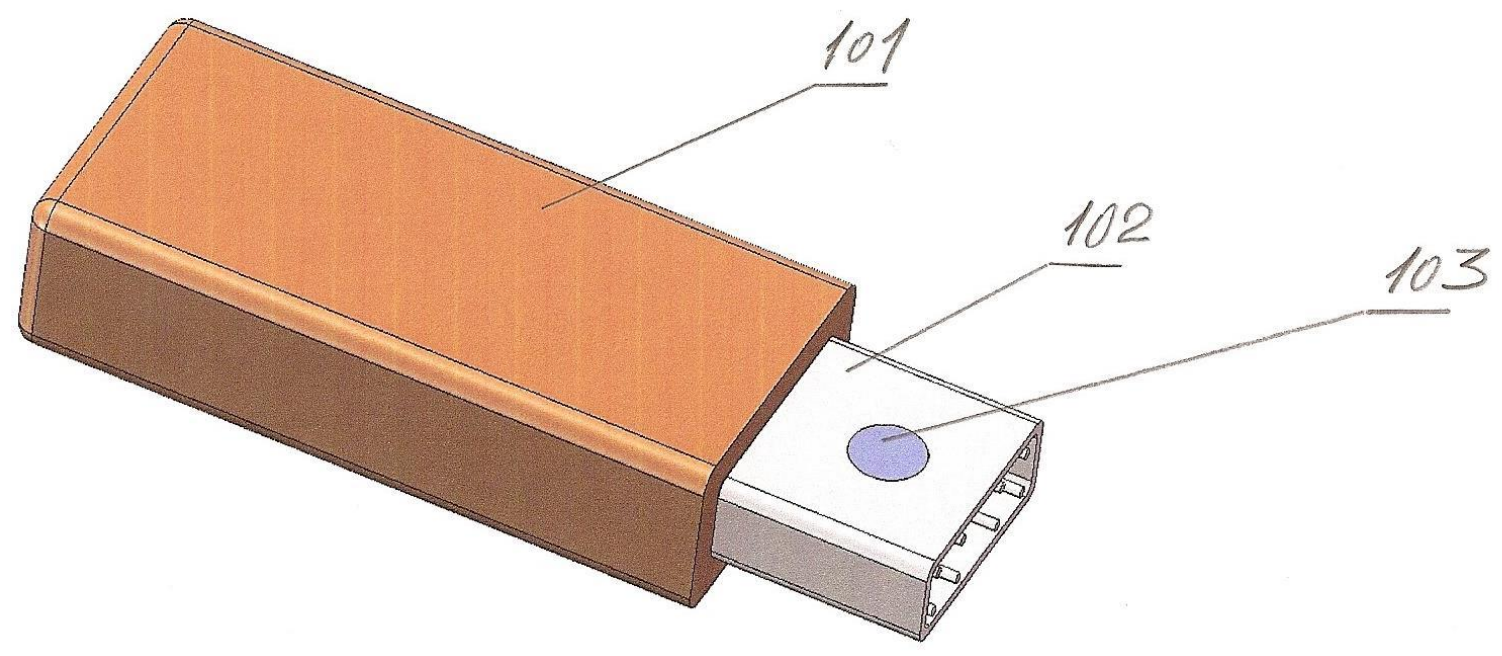

Figure 11, - a three-dimensional model of a storage medium is shown in the drawing 


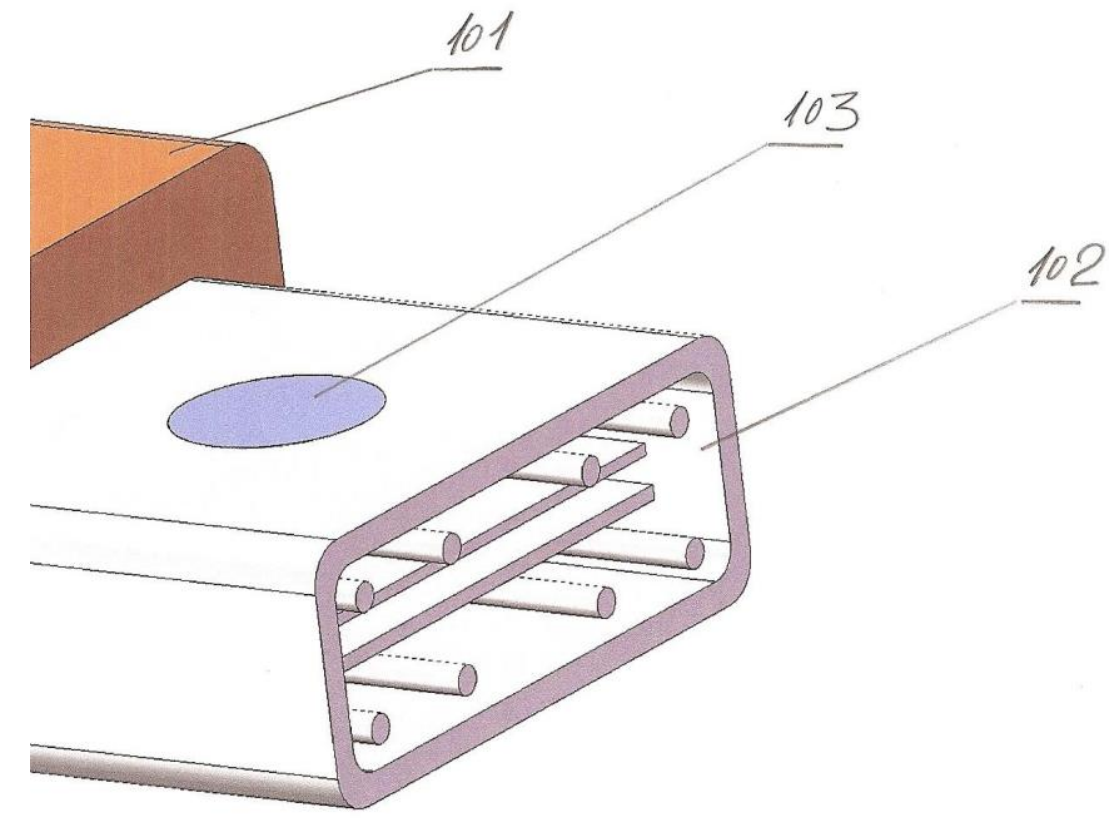

Figure 12, - a fragment of the three-dimensional model of a storage medium in the region of a flat plug connector with a coding and decoding disk is shown in the drawing

The following are figured in drawing 11 and 12:

- 101, - a casing of the storage medium;

- 102, - a flat plug connector;

○ 103 , - a coding disk.

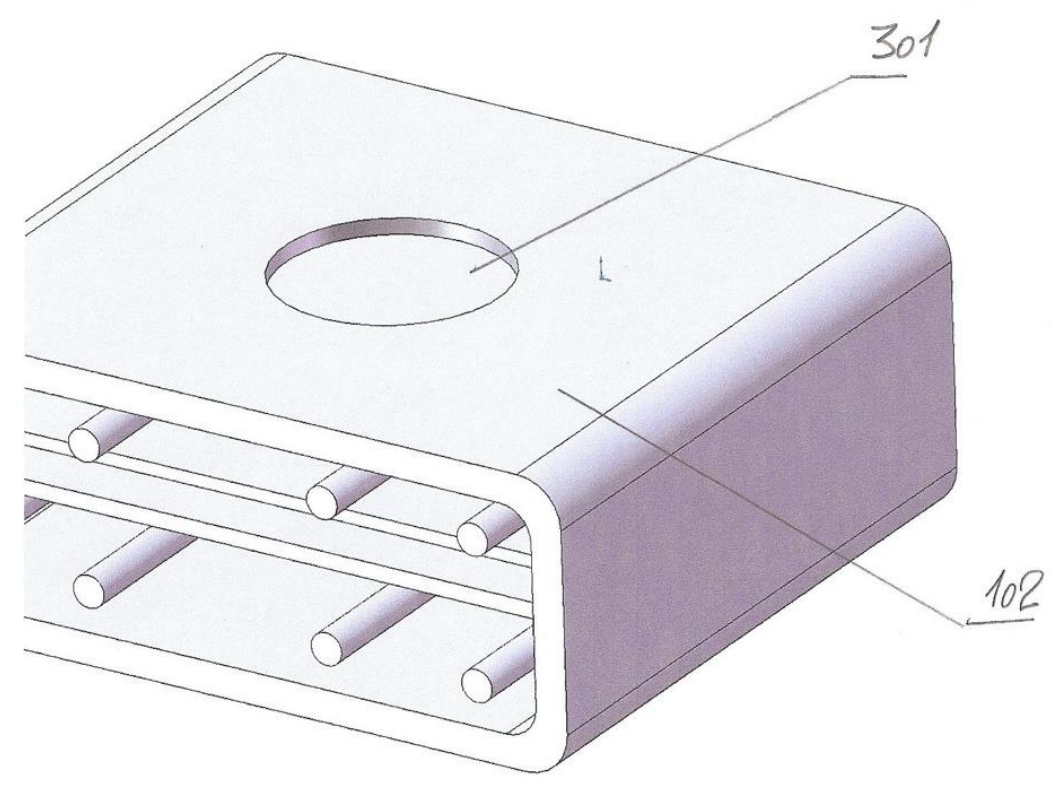


Drawing 13, - a fragment of the three-dimensional model of a storage medium in the region of a flat plug connector with a coding and decoding disk is shown in the drawing prior to installation of the coding and decoding disk

The following are figured in the drawing:

- 102 , - a casing of the flat plug connector;

- 301 , - a bore in the casing for installation of the coding disk in any design option.

As it is seen from all presented models of the storage medium in figures 10,11, 12 and 13, in terms of design the coding and decoding system is quite simple, which enables its integration even on storage media made without such device and such functions.

This implies an additional opportunity of using the newest technologies for increasing the marketing potential of the new product. The following technology can be cited as an example:

The method of production of a module for installation, cooling, management and control of energetically saturated optical and electronic systems including:

- treatment of a surface of a steel tape (roll) (metal can be specified instead of steel);

- application of photoresist;

○ development of photoresist;

- a rapid stream-like electrochemical coating with nickel in a directed flow of an electrolyte (2-3 microns thick);

- a rapid stream-like electrochemical coating with copper in a directed flow of an electrolyte (25-35 microns thick).

"Since this technological phenomenon is the main basic distinction and makes a package of substantial advantages of the proposed innovative method", - the author considers it necessary to give some explanation to this phenomenon.

The rapid stream-like electrochemical coating is a developed galvanic process in a selectively oriented directed flow of an electrolyte with a constant, electrolyte updating electrolyte recirculation system, which (the recirculation system) includes:

- a capacity with an electrolyte with certain parameters of a maintenance mode of the electrolyte in the capacity, such as:

○ concentrations of nickel and copper in the electrolyte

$\circ$ temperatures of the electrolyte

- acidity or alkalinity level of the electrolyte density of the electrolyte

- electric resistance of the electrolyte (conductivity)

0 owing to the abovementioned advantages of the technology and used materials there is no need in using organic additives to the electrolyte - clarifying agents;

○ a pump with a filter; 
- anode for stream-like metallization, which has soluble in this type of the electrolyte and insoluble in this type of the electrolyte components.

Components installed consecutively in the direction of the motion of the electrolyte, notably that the insoluble component was made of a composite, graphite and current-conducting tissue (like ETHANE). The insoluble component is located in parallel to a metallized area and last in the direction of the motion of the electrolyte and first before a covered surface (cathode). Both components are connected to a positive electrical potential as well as both components have a selective regulated hydraulic permeability for the electrolyte.

In the anode there is also a system of even distribution of the electrolyte on the plane of the soluble component, which is automatically repeated on the insoluble component and consequently on the metalized area - cathode:

○ photoresist removal;

○ iron etching, from one side, for the half of the thickness of the steel tape

- removal of etching products from the surface by aerodynamic and thereupon hydrodynamic effect;

○ pressurization by a free-flowing polymer compound, - in a such technologic procedure:

○ filling with a monomer

- subsequent layer-by-layer polymerization

○ thermal stabilization;

0 iron etching from the second side of the tape (with the same distinctions);

○ pressurization from the second side (with the same distinctions);

- application of a protector on electrically conductive structures;

- a coating in a vacuum of all heat-conducting structures - with a layered system of semiconductive nano-structured polycrystalline diamond films.

Such technological addition to traditional techniques and methods enables to expand spheres of introducing methods of marketing of the new product substantially.

\section{Organization of corporate security systems}

The proposed technology during organization of the security system of information flows within one corporation ensures security at several system levels, including both tracing in the real time system of a state and location of each disk, which is in the corporation.

When using the proposed methods of coding, for security of information on mobile external storage media, it is supposed to obtain the same advantages, as when using on optical storage media and information tanks. 


\section{Changes in the structure and boundaries of using the product created as the result of project implementation}

Thus, based on analogous solutions it is possible to create at least two projects with a large number of applications in each, - a technology project for coding optical information tanks represented by a disk, including a relevant analytically-sensing device, which may in its turn have a variety of applications in different spheres and branches.

And the project for coding and security of information on mobile external storage media, including a relevant mobile or stationary sensor measuring-analytically-comparative device, also having a variety of applications and design-models.

\section{Additional devices and systems, which can be created on the basis of the same fundamental technologic solutions}

Upon demand of a consumer of projects the project package may include a section, relating to additional or special devices, using which the whole corporate system of safeguard and security of information flows is formed within one corporation or a group of corporations or (according to Russian specificity, - state corporations) individual scientific research establishments, research institutes and large institutions in the healthcare system.

An information security system can be created as a special product not only in the sphere of storage, but in the operative sphere, when transferring commands and signals in the setting of army units and commands, and especially, in the setting of the - sea fleet.

In modern conditions, when information is concentrated in relatively very small sizes and capacities of devices for its storage, possible harm from unauthorized or criminal entry to these arrays of information can be prevented or localized using creation of a special infrastructure of the specified security systems, which can be standardized within the specificity of this ministry, main directorate or structural corporate units and enterprises of the lower organizational level.

A series of drawings, - from drawing 14 to drawing 20 shows, as an example, applications of coding and decoding technologies in identification of a one-use tool in laser medical devices. 


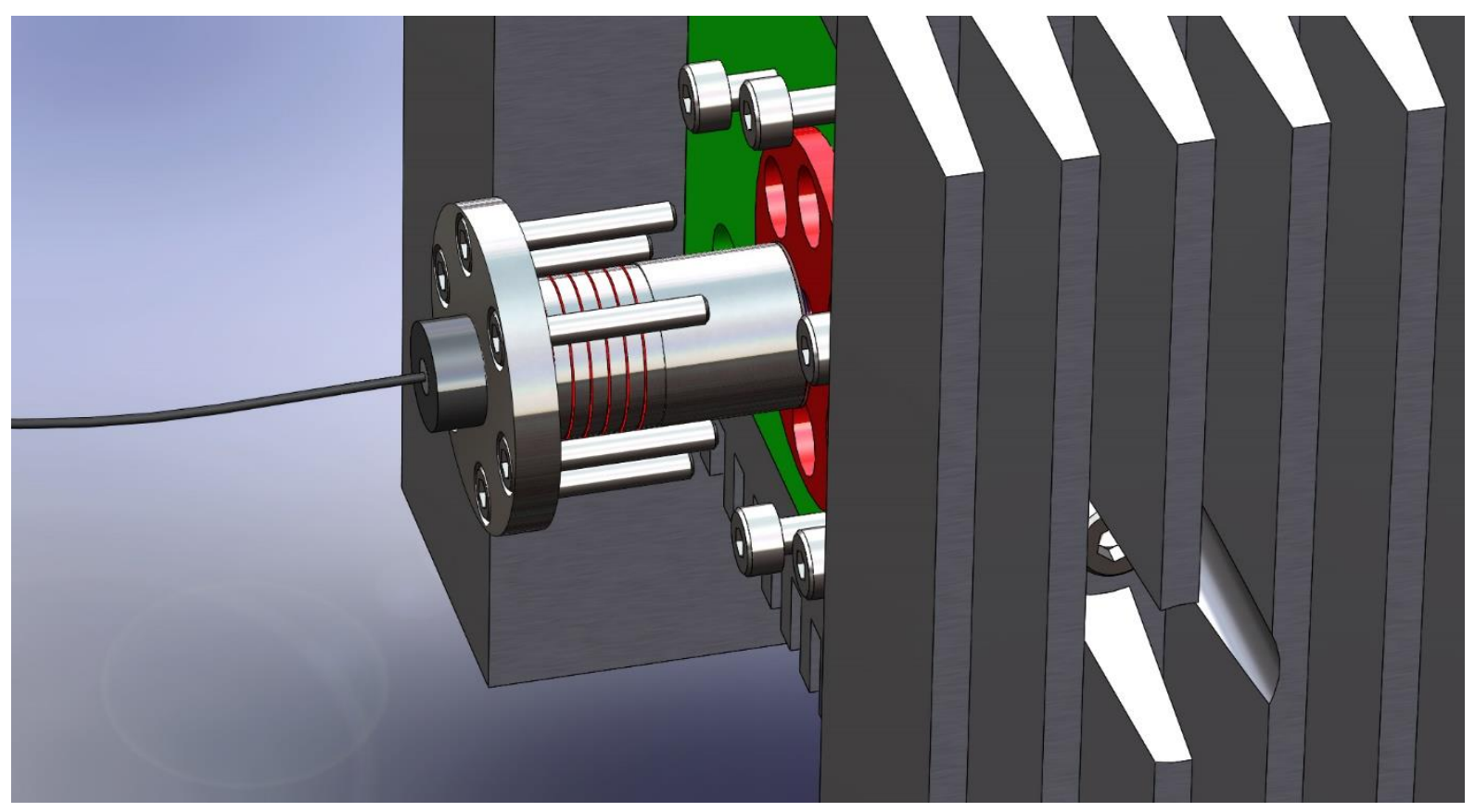

Figure 14, - a three-dimensional model of a docking device of a laser endoscope for installation and identification of one-use tool is shown in the drawing

Such combination of functions increases efficiency of operation of the system and substantially simplifies a design of the unit, which in its turn decreases its value and costs for operation and repair.

In its turn decrease in expenses while improving the quality gives additional warranties for more confident marketing for the new product.

\section{Confidentiality of information}

More in detail (in volumes going beyond this publication and illustrative materials to it) all necessary information can be provided during documentary and legal formulation of intentions of a potential consumer or a partner, after signing confidentiality agreements with him (by a mutually agreed, acceptable for both parties legal form).

A magnetic resonance method was applied for more complete presentation of existing physical bases of performance of coding and decoding operations of optical disks, a brief description of which is given below. 


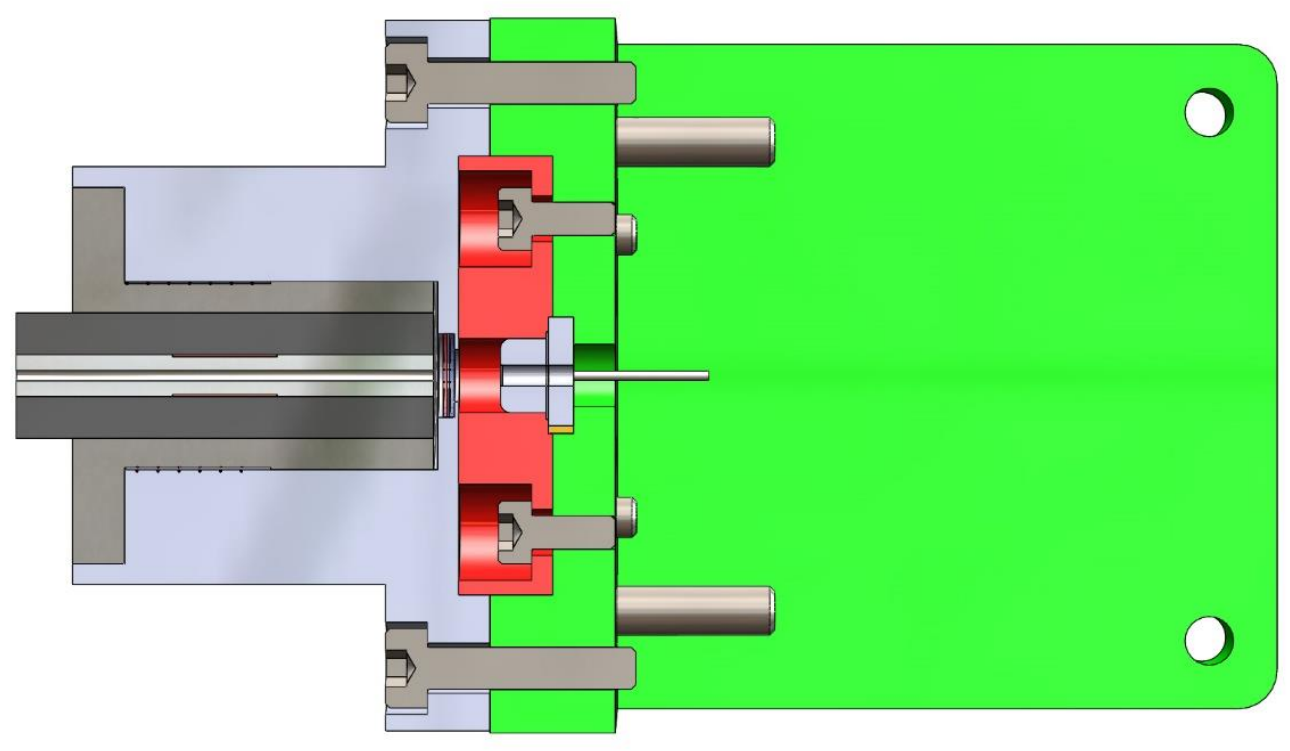

Figure 15, - a three-dimensional model of a docking device of a laser endoscope for installation and identification of one-use tool is also shown in the drawing. The model is shown in the axial section, where the system of functional interaction between a laser diode and optical cable, as the axis of one-use tool is seen

The system is maximally easy for reproduction and operation

\section{Brief description of the resonance method}

The method provides for creation of an alternating electromagnetic field in space, in which a studied sample is located. This field is an intermediate between a resonance circuit and the test sample.

On the one hand, the resonance circuit is an emitter (radiator) of this field, and on the other hand - an acceptor (a sensing element), of those changes in the electromagnetic field, which the tests sample makes.

Even in the absence of the test sample, the alternating electromagnetic field created by a solenoid is the sum of two electromagnetic fields, which change in antiphase to each other.

One field is generated by change of magnetic induction of the solenoid and have an electric curl field as a consequence (Maxwell-Faraday equation). The other one is generated by change of the electric field created by difference of potentials between extreme the most remote from each other solenoid coils (if the sample is placed inside of the solenoid) or difference of potentials between the closest to the surface coil of the measured sample and the sample itself 
(if the sample is located against the solenoid face), and has a whirling magnetic field as a consequence (Ampère's circuital law with Maxwell's correction).

Affected by the external alternating electromagnetic field in the test sample, depending on its nature, such electric phenomena as linear and whirling currents of conductivity, linear and whirling currents of displacement, as well as linear and whirling ionic currents (ordered motion of ions) may be induced.

In accordance with the superposition principle of fields these electric phenomena introduce distortions to the external alternating electromagnetic field.

These distortions are perceived by the solenoid of a resonance sensor. A resonance circuit that includes this solenoid, changes its behavior similar to that, as if it included additional elements: a condenser, inductivity and a resistor.

The aggregate of additional capacity, inductive and active resistances represents an additional impedance contributed by the test sample to the system, this attribute is measured by the resonance sensor.

Changes of parameters of the resonance circuit are reflected in the change of its amplitudefrequency response, namely, resonance frequency and amplitude of the circuit change.

Studying these changes, one may estimate impedance of the tests sample.

\section{Principle of processing of data obtained from resonance sensors}

The resonance sensor allows determining a value of a resultant impedance of the studied sample at an operational frequency of this sensor (see "Brief description of the resonance method").

This value by its self is low informative.

But everything ultimately changes, if we have a set of sensors with different operating frequencies.

In this case there is an opportunity of using a unique natural phenomenon observed in all types of substances: non-organic, organic and biological. This phenomenon resides in the fact that a substance changes its specific impedance depending on a frequency of an electric field affecting it, and this change depends upon a composition of a studied substance.

A rapidly developing in the recent time scientific field called Magnetic resonance spectroscopy - impedance spectroscopy studies this phenomenon and actively uses it.

In English-language sources it is more often called Electrochemical Impedance Spectroscopy (EIS) (impedance spectroscopy). 
Magnetic resonance spectroscopy - impedance spectroscopy is a method of researching various objects, based on measurement and analysis of dependences of impedance upon frequency of the alternating current.

Different objects and processes are characterized by different dependences of active and reactive impedance on frequency, which makes possible solving an inverse problem obtaining information about these objects and processes through analysis of frequency characteristics of their response at the alternating current.

The fact that change of impedance while changing frequency depends upon a composition of the substance, allows detecting changes and influence of each component on resultant impedance of the substance at different frequencies.

After determining weight coefficients of influence of relevant components on resultant impedance of the substance on each of operating frequencies of resonance sensors, it is possible based on sensor readings, solving a system of linear equations to obtain information about a concentration of studied components.

A right choice of operating frequencies of sensors makes a great impact on accuracy of this method.

By scanning in a wide range of frequencies it is necessary to determine the most inherent for each component frequency domains, i.e frequencies, at which a component gives the largest response.

Traditional impedance spectroscopy in its researches uses ac voltage source, which affects the studied sample by a contact method, whereas electric current appears in the circuit, a value and phase shift of which depend upon impedance of the sample.

Results are displayed as a rule as Lissajous patterns or Nyquist diagrams.

With such researches it is difficult to achieve high sensitivity and accuracy of measurements. The proposed methodology, in which measurement of impedance is made using resonance circuits, has a significantly higher sensitivity and accuracy, furthermore it is contactless.

There exist certain technical difficulties of creating an oscillating circuit with a resonance frequency readjusted in a wide range, therefore it will be necessary to use traditional Magnetic resonance spectroscopy - impedance spectroscopy for searching for frequencies "inherent" for components.

When inherent frequencies are found and resonance sensors are created for these frequencies, a system of component monitoring created based on these sensors will have exclusive sensitivity and accuracy. 


\section{Interference immunity}

Such "mechanic" parameters as VISCOSITY, DENSITY, TRANSPARENCY, PRESSURE (if a medium is incompressible) shall not make any impact on measured electric parameters of a substance.

MOTION SPEED IN A PIPLELINE and TURBULENCE - these phenomena are too slow in order to make an impact on "megaherz" processes of impedance measurement. HARDNESS is a chemical parameter, which is fully determined by components that are included into the substance.

Temperature, as a rule, makes an impact on a value of impedance, but measurement of temperature and its recording while impedance measuring is not a complicated technical task.

1. Principled foundations of secure coding of optical storage media or information tanks, predominantly in the form of a disk, transparent for luminous flux, coming from an exit optical system of a single-mode laser diode, having standard required dimensions, - outer diameter, - 120 millimeters, and thickness, -1,2 millimeter.

The disk is pasted of two halves, each 0,6 millimeter thick; a coating is applied on one of halves of the disk on a ring, the outer dimeter of which is 120 millimeters, and the inner dimeter of which is 118 millimeters; the thickness of the coating varies in a range from 1 micron to 10 microns with an interval 100 angstrom.

Suh accuracy is fully ensured by properties and parameters of a technology of rapid electrochemical coatings and as well as ensures high accuracy of identification in operation, not introducing mistakes related to inaccuracy of code measurements.

1.1. Conceptual foundations of coding reside in the following principle: - a coding signal is produced from a reaction of a sensing unit or a group of sensing units to a thickness of a ring coating on a disk, comparison of the obtained signal to a statistical reference of this signal, - equivalent of the resonance reaction of sensing units to the thickness of the coating, specific indicators of the coating material, conductivity of the coating material, density of the coating material, electric resistance of the coating material.

1.2. Instead of one of points of the group combination, a signal from a decoding sensing unit of the secure coding system is introduced to the servo-marking system of the formatted disk, which, as a rule, has a type of group combinations of servo-points on information tracks of the disk, and in case of coincidence of an integrated signal from three sensing units with pre-set parameters of the signal, the servo system of a disk drive starts orienting a focus of the laser on the information track, and thus the system starts the reading or recording process on the optical disk.

1.3. In case of non-coincidence of the signal from sensing units with a statistical form of the signal in the memory of the disk drive processor, the servo system of the disk drive does 
not orient and does not stabilize a trajectory of a beam focus of the laser diode on the information track of the disk and reading or recording on the disk become impossible.

\section{OPTIONS OF IDENTIFICATION OF A DISK IN A DISK DRIVE:}

2.1. Identification of a disk in a disk drive can be run using measurement of a coating thickness in a real-time mode, comparison of results of measurement to a statistical value of this parameter stored in the disk drive processor and delivery of the signal to a comparator in the disk drive processor.

2.2. The identification process can be run during disk rotation or during installation of the disk to the disk drive.

2.3. During identification during installation of the disk to the disk drive, negative results of identification do not allow switching of any structure of the disk drive, and on the contrary a positive signal of identification switches on necessary structures of the disk drive.

2.4. Similar logics and procedure of operation take place in the coding and decoding system of one-use tool in different devices and technical systems of any level.

2.5. Such design interrelation substantially standardizes all technological transitions of the identification, coding and decoding process, irrespective of a specific type and scope of application of the product, and only depending on design features and characteristics of a special unit for coding and decoding.

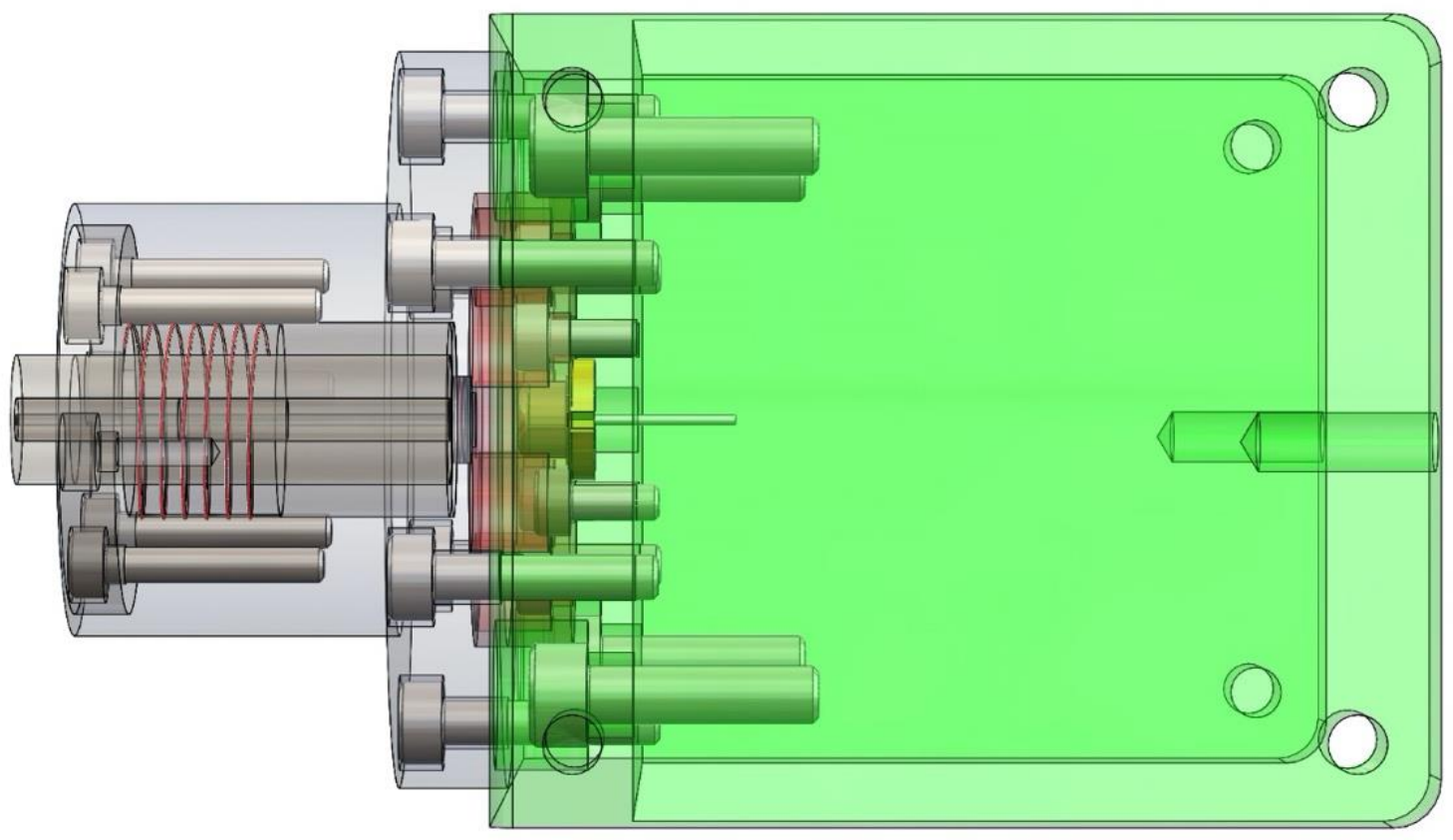

Figure 16, - a three-dimensional model of a docking device of a laser endoscope for installation and identification of one-use tool is also shown in the drawing. The model is shown in a transparent version, top view, where a system of functional interaction is seen between a laser diode and an optical cable, as an axis of one-use tool. The solenoid of the 
sensing unit from the signal of which coding and decoding of one-use tool is performed is visible

\section{DESIGN OPTIONS OF DISK DRIVES:}

3.1. Elements of the secure system of resonance coding - decoding can be without any design or circuit limitations, embedded to any existing today design of the disk drive, implementing all known technologies of optical memory.

3.2. Existing disk drives can also be modified for assembling of the system of microsensors, by insetting a sensor micromodule to a bearing structure of the disk drive casing.

3.3. If necessary, a coating can be performed out on already existing disks.

\section{APPROXIMATE PROCESSING ROUTE OF MANUFACTURING A DISK WITH A CODING COATING:}

4.1. Special technologies and equipment are not required for manufacturing an optical disk with a secure coding coating.

4.2. Upgraded processing equipment, which is currently used, can be used for manufacturing.

4.3. Application of a coding coating can be combined with manufacturing a copy of the disk in a press mould using a master disk with an identification point in the formatted servomarking system, which in such manner will be pressed on each information track, - and there are more than 37000 in an ordinary optical disk.

\section{OPTIONS OF USING DISKS WITH A SECURE COATING IN SYSTEMS OF OPTICAL MEMORY OF CORPORATE CLIENTS:}

5.1. An approximate scheme of using disks with protective coding-decoding in corporate clients provides for manufacturing a certain amount of disks for each such client with parameters of thickness and coordinates of microsensors inherent only to this client.

5.2. Design and technical characteristics of a sensor micromodule can also be modernized based on client's wishes, but in accordance with control parameters of the secure coding coating on disks. 


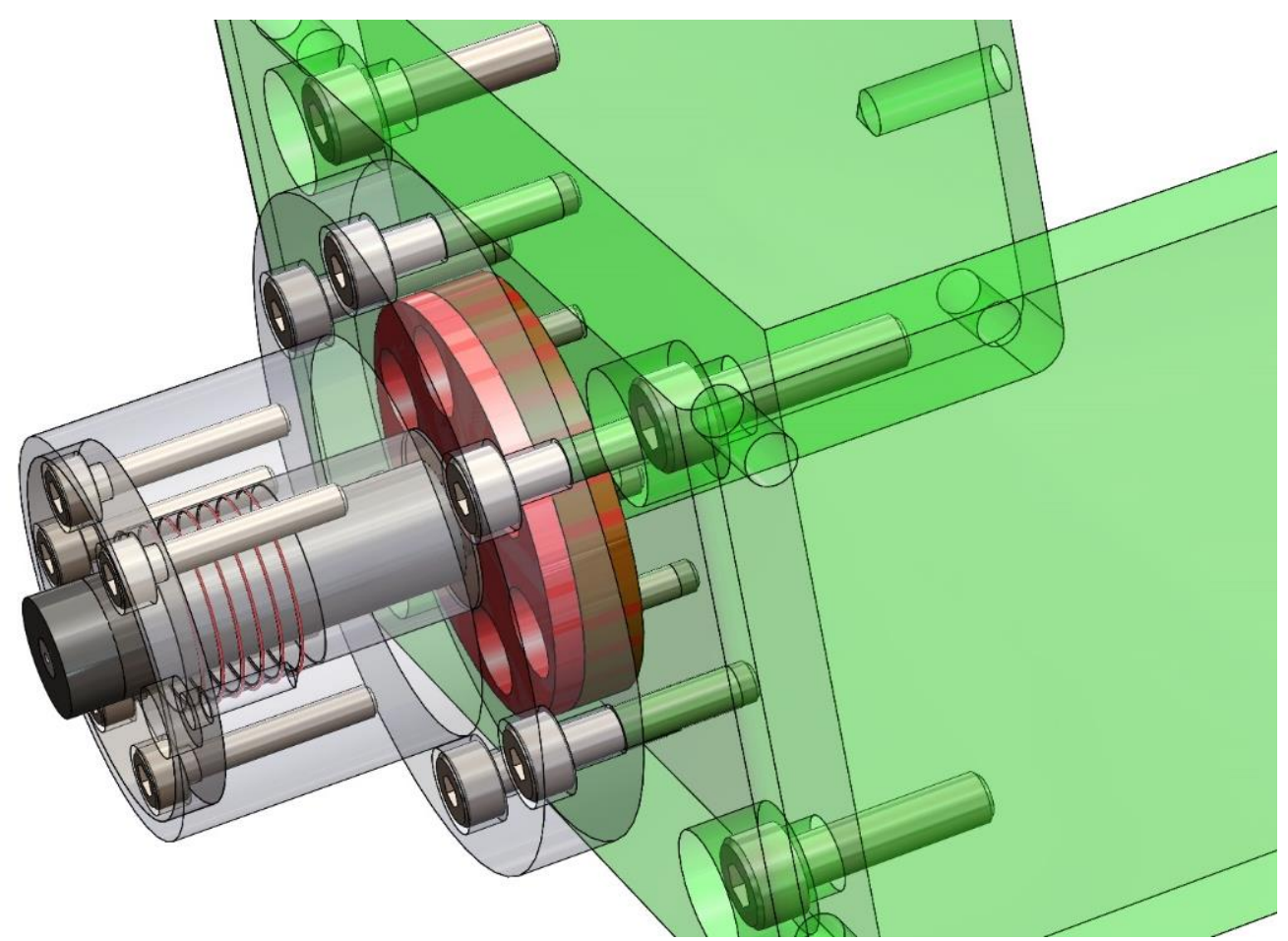

Figure 17, - a three-dimensional model of a docking device of a laser endoscope for installation and identification of one-use tool is also shown in the drawing. The model is shown in a transparent version, isometric view, where a system of functional interaction is seen between a laser diode and an optical cable, as an axis of one-use tool. The solenoid of the sensing unit from the signal of which coding and decoding of one-use tool is performed is visible

\section{OPTIONS OF USING DISKS WITH SECURE CODING IN SYSTEMS OF HOUSEHOLD RADIO TECHNOLOGY:}

6.1. Disks with secure coding can be used in Blu-Ray and HD DVD systems.

Apart from that, the system of secure coding can be applied in new developments and technologies of optical digital memory including disks with especially high density of recording, multi-layer disks, monolithic optical disks with the memory capacity 1 and more terabyte.

6.2. While manufacturing disks, necessary indication to servo-marking can be introduced in the pressing process.

A servodrive of the disk drive starts orientation of a focal point of the laser beam only in case of coincidence of a coding signal from the coding and decoding system produced by the system of three micro-sensors, which using magnetic resonance methods, compare a thickness of the coating to a reference and in case of coincidence of parameters of the signal with the reference at least in two sensors, the obtained signal is added to the system of symbols and marking points of servo-marking, reading which the servodrive 
of the disk drive starts stabilizing a focus of the laser on a necessary track in a field of disk recording.

\section{OPTIONS OF USING DISKS WITH A SECURE COATING IN PERSONAL COMPUTERS:}

7.1. A manufacturing technology of disks for personal computers is similar to the manufacturing technology of such kind of disks for other options of optical memory.

7.2. A methodology of using disks with secure coding is built up based on a type of a computer, extent of its saturation and capacity, speed and etc.

7.3. Especially important is a possibility of using processes and technology of secure coding in created hybrid disks, combining in itself a hard disk with an optical disk.

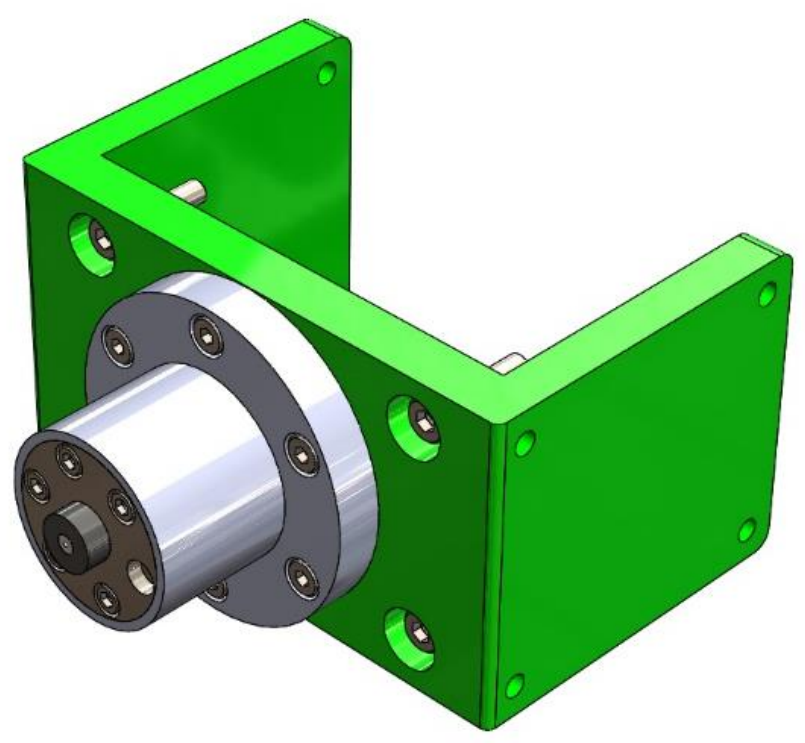

Figure 18, - a three-dimensional model of the casing of the plug-in connector docked to the main detail bearing the laser diode (designated with the green color in the drawing) is shown in the drawing

\section{Proposal on the system of obtaining professional information from Internet}

An optical disk is the main tool, on which a coding coating is applied in a ring zone, in which there is no information recording.

A microsensor is an auxiliary tool, which is embedded into the disk drive. 
A signal from the microsensor is produced while measuring a thickness of the coating; accuracy of measurement, - 100 angstrom and this is value for which each group of disks differ from the other group.

A signal from the microsensor is a code for entering arrays of information placed in Internet.

A software shall make it possible to identify a signal from a microsensor and in case of coincidence of the signal with the reference one to open arrays of information and in the process of its downloading to continue controlling fidelity of the signal till completion of the information downloading process.

This enables preventing replacement of a disk during recording to a non-licensed one.

It is impossible to falsify such disk, since the thickness of the coating is determined during manufacturing and, even having such disk, it is impossible to use it, without a microsensor configured to a strictly definite nature of the signal.

Disks and sensors can be manufactured at any existing today production facility of optical disks; disks can be manufactured in series by 100 - 250 pieces with a similar thickness of the coding tape and with a set of sensors.

Every user may acquire one or several series of disks and use them while working with Internet.

In the same principle programs and other information can be sent to users, only in the inverse order, which warrants full confidentiality and protection while being in Internet from unauthorized messages and viruses.

This is very general information if course, if you readers consider it deserving attention, then the author could elaborate this project.

Taking into account the fact that the mechanical part of this project is as a matter of principle implemented, this project is a software, which may become a basis of the project in this direction. 


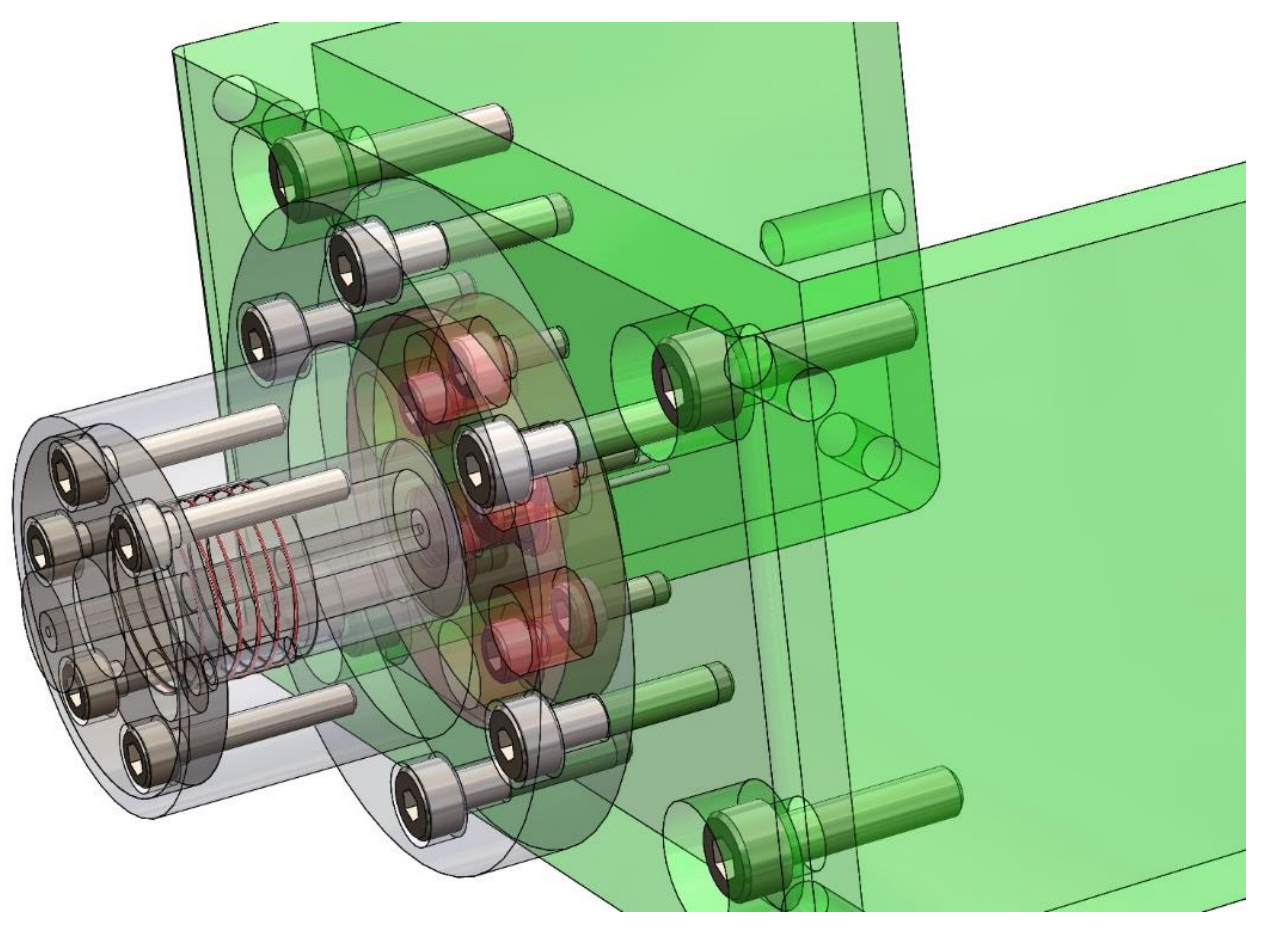

Figure 19, - a coaxial casing of a plug-in connector with a coding and decoding device as a coaxially installed solenoid is shown in the drawing, which jointly with an impulse generator produces a coding and decoding signal and after its passage sorts and transmits a resonance signal to an analytical unit

\section{Advantages of the proposed technology, responding to the essence of problems detected on the market of systems of optical storage media:}

1. There is a variety of options of thicknesses of coding coatings, which allow having a variety of options of a security code, as distinguished from well-known technologies, which have only one option of the code.

2. In the process of application of a coating, a control technology is used fully identical to the decoding technology, which allows fully controlling quality of coding in the process of disk manufacturing, without removal of the disk from a conveyor, as distinguished from existing technologies, in which a disk for control should be removed from the conveyor and installed in a control device.

Thus, control is selective, and in the proposed technology, - 100\% control, which excludes production of defective disks, which are found in existing technologies only during operation.

3. In the proposed technology there is a possibility of coding all categories and types of disks irrespective of a format of recording and reading, as distinguished from existing technologies, in which coding depends upon a format of recording and reading of the disk. 
4. In the proposed technology a coding coating can be a basis for a personal secret code or cipher, which existing technologies do not have.

5. In the proposed technology a sensing unit of decoding and identification is mobile and may have several options of delivery, including an autonomous option, not related to a disk drive, and in the existing technologies the decoding system is installed only in disk drives; thus it is possible to control availability and correctness of coding only in the process of installation of the disk to the disk drive, and in the proposed technology it is possible to control and identify a code out of the disk drive, for example in shops or in entrance control posts of enterprises and institutions, which is especially important for ensuring full nondisclosure mode.

6. In the proposed technology decoding excludes any dependence upon optical systems of the disk drive, but results of decoding may change operation of optical systems, for example a servo drive for orientation and control of a focus position of a reading or recording laser, as distinguished from existing technologies, in which a decoding process fully depends upon optical elements of the disk drive, which complicates its design and severely reduces reliability.

7. The proposed technology has several hierarchies of a fundamental operating scheme, has a flexible algorithm and can be built into any security system of optical memory including to hybrid information-carrying media, which have apart from an optical component, media constructed on other basic principles; existing technologies do no have specified flexibility.

8. The proposed technology allows using a code of a disk as an entrance password for entering professional arrays of information of Internet, which existing technologies do not have.

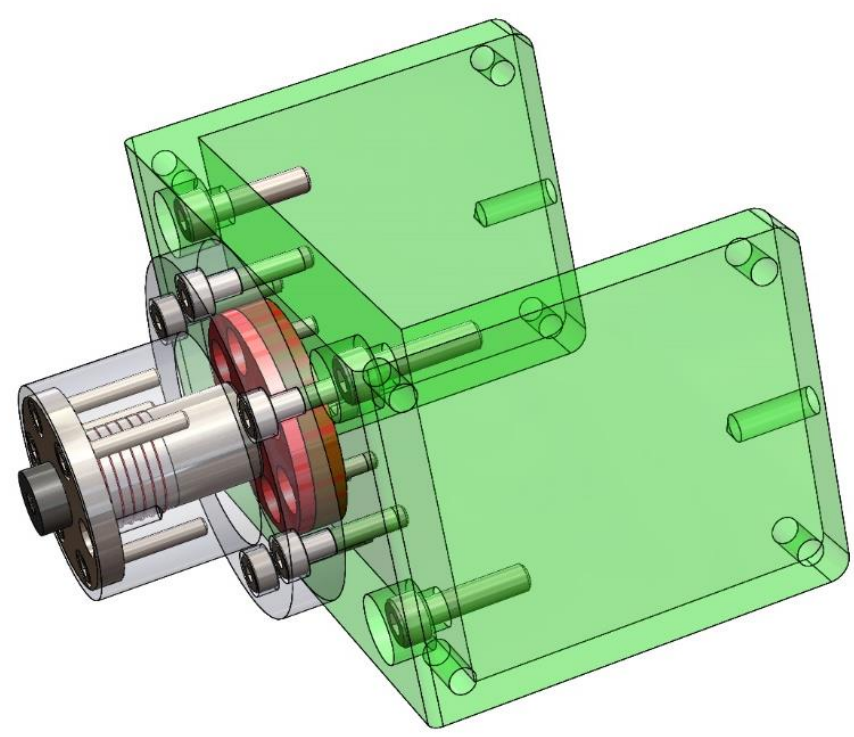

Figure 20, - a module of a laser diode with a coding and decoding plug, containing a sensing unit-solenoid located coaxially to a plug connector of one-use tool is shown in the 
drawing, notably that the connector plug is made as a plug, a wall thickness of which is a key to a ciphering code, which is identified using resonance spectroscopy

Technology and design solution of such an integrative sensing unit, allows its building into practically any technical system, where connection, for example of one-use tool is performed using a plug-in connector.

The following general marketing strategy is developed preliminarily according to results of a complex analysis using algorithms of the patenting and licensing strategy.

\section{Preliminary determination of a core product of the project ad its functional and operating features and characteristics:}

1. The main sector of the market, - corporate clients:

- Banks and financial companies;

- Industrial corporations;

- Scientific Research Laboratories;

- Transport companies, railway station buildings, airports and sea ports;

○ Large distribution networks;

- Municipal services;

- Governmental organizations and institutions;

- Large medical institutions;

- Insurance companies;

- Branches of arms of armed forces;

$\circ$ Police and intelligence services.

2. Product characteristic (as an example):

- A product of the project (main), - an optical disk with a coding ring with an embedded sensor module, as a rule consisting of three microsensors;

- If necessary, the sensor module can be supplied without a disk drive;

- If necessary, a company running the project, can provide services for corporate clients, organizing bringing a coding system and information security into action, - on a turnkey basis. 


\title{
LIST OF REFERENCES, PATENT AND LICENSE MATERIALS
}

\section{ANNEX 1}

United States Patent Application

20190281321

Kind Code

ZHAO; Xin ; et al.

September 12, 2019

\section{METHOD AND APPARATUS FOR VIDEO CODING}

\begin{abstract}
Aspects of the disclosure provide method and apparatus for video coding. In some examples, an apparatus includes processing circuitry. The processing circuitry determines an intra prediction mode for generating a prediction image of a current block, determines one or more primary transforms according to signaling information that is extracted from a coded video bitstream, and determines a secondary transform according to the determined intra prediction mode and the determined one or more primary transforms. The processing circuitry also reconstructs a residue image of the current block based on one or more coefficients of an input block extracted from the coded video bitstream, the determined one or more primary transforms, and the determined secondary transform. The processing circuitry then reconstructs an image of the current block based on the prediction image and the residue image of the current block.
\end{abstract}

\section{ANNEX 2}




\title{
SHARING OF MOTION VECTOR IN 3D VIDEO CODING
}

\begin{abstract}
Joint coding of depth map video and texture video is provided, where a motion vector for a texture video is predicted from a respective motion vector of a depth map video or vice versa. For scalable video coding, depth map video is coded as a base layer and texture video is coded as an enhancement layer(s). Inter-layer motion prediction predicts motion in texture video from motion in depth map video. With more than one view in a bitstream (for multiview coding), depth map videos are considered monochromatic camera views and are predicted from each other. If joint multiview video model coding tools are allowed, inter-view motion skip is used to predict motion vectors of texture images from depth map images. Furthermore, scalable multiview coding is utilized, where inter-view prediction is applied between views in the same dependency layer, and inter-layer (motion) prediction is applied between layers in the same view.
\end{abstract}

\section{ANNEX 3}

United States Patent Application

20190273948

Kind Code

Yin; Hujun ; et al.

September 5, 2019

METHOD AND SYSTEM OF NEURAL NETWORK LOOP FILTERING FOR VIDEO CODING

\section{Abstract}

A method, system, medium, and article provide neural network loop filtering for video coding with multiple alternative neural networks.

\section{ANNEX 4}

United States Patent Application

20190273932

Kind Code

HAQUE; MUNSI ; et al.

September 5, 2019

VIDEO CODING SYSTEM WITH TEMPORAL SCALABILITY AND METHOD OF OPERATION THEREOF 


\begin{abstract}
A method of operation of a video coding system includes: receiving a video bitstream; extracting a video syntax from the video bitstream; extracting a hypothetical reference decoder (HRD) fixed syntax from the video syntax; extracting a HRD variable syntax from the video syntax; extracting a temporal layer from the video bitstream based on the HRD fixed syntax and the HRD variable syntax; and forming a video stream based on the temporal layer for displaying on a device.
\end{abstract}

\title{
ANNEX 5
}

United States Patent Application

20190268098

Kind Code

A1

CHUN; Jinyoung ; et al.

August 29, 2019

DATA TRANSMISSION METHOD IN WIRELESS COMMUNICATION SYSTEM AND

DEVICE THEREFOR

\begin{abstract}
A downlink (DL) multi-user (MU) transmission method in a wireless local area network (WLAN) system, the DL MU transmission method including receiving a DL MU physical protocol data unit (PPDU) including a physical preamble and a data field from STA (Station) and transmitting the ACK frames in response to the DL MU PPDU to STA. In addition, the data field includes at least one medium access control (MAC) protocol data unit (MPDU), the at least one MPDU includes a MAC header and a MAC frame body, wherein the MAC header includes acknowledge (ACK) indication information, the ACK indication information includes frequency resource allocation information for an uplink (UL) MU orthogonal frequency division multiple access (OFDMA) transmission of ACK frames and modulation and coding scheme (MCS) level information, and the frequency resource allocation information includes an index value indicating a resource unit allocated for the UL MU OFDMA transmission of the ACK frames, and the resource unit corresponds to a 26-tone resource unit, a 52-tone resource unit, a 106-tone resource unit, a 242-tone resource unit, a 484-tone resource unit, or a 996-tone resource unit.
\end{abstract}

\section{ANNEX 6}

United States Patent Application

20190268016

Kind Code

JANSSON TOFTG RD; Tomas ; et al.

August 29, 2019 


\begin{abstract}
A method for partitioning of input vectors for coding is presented. The method comprises obtaining of an input vector. The input vector is segmented, in a non-recursive manner, into an integer number, N.sup.SEG, of input vector segments. A representation of a respective relative energy difference between parts of the input vector on each side of each boundary between the input vector segments is determined, in a recursive manner. The input vector segments and the representations of the relative energy differences are provided for individual coding. Partitioning units and computer programs for partitioning of input vectors for coding, as well as positional encoders, are presented.
\end{abstract}

\title{
ANNEX 7
}

United States Patent Application

20190261010

Kind Code

A1

Luo; Ning ; et al.

August 22, 2019

METHOD AND SYSTEM OF VIDEO CODING WITH REDUCED SUPPORTING DATA SIDEBAND BUFFER USAGE

\begin{abstract}
Methods, systems, and articles of video coding with reduced supporting data sideband buffer usage.
\end{abstract}

\section{ANNEX 8}

United States Patent Application

20190253706

Kind Code

ZHAO; Liang ; et al.

August 15, 2019

METHOD AND APPARATUS FOR USING AN INTRA PREDICTION CODING TOOL FOR INTRA PREDICTION OF NON-SQUARE BLOCKS IN VIDEO COMPRESSION

\begin{abstract}
A method for video decoding includes determining whether an angular intra prediction mode for a current block is a wide angle mode that is spaced apart from a vertical mode and a horizontal mode by an angular distance that is more than a predetermined threshold. The method further includes, in response to the determination that the angular intra prediction mode
\end{abstract}


is the wide angle mode, determining a non-wide angle mode that corresponds to the wide angle mode, the non-wide angle mode being spaced apart from the vertical mode or the horizontal mode by an angular distance that is less than or equal to the predetermined threshold. The method includes determining whether a coding tool is specified for the determined corresponding non-wide angle mode, and, in response to the determination that the coding tool is specified for the determined corresponding non-wide angle mode, using the coding tool in a decoding process.

\title{
ANNEX 9
}

United States Patent Application

20190181979

Kind Code

Wang; Ying ; et al.

June 13, 2019

\section{ADJUSTED FRACTALLY ENHANCED KERNEL POLAR CODES FOR ACHIEVABLE SIGNAL-TO-NOISE RATIO SPIKE MITIGATION}

\begin{abstract}
Methods, systems, and devices for wireless communications are described. In some systems, wireless devices may implement adjusted fractally enhanced kernel polar coding. An encoder may receive a number of information bits and a block size for transmission, and may append an additional number of information bits to the information bits for transmission. The encoder may perform a recursive bit allocation process to allocate the aggregate set of information bits between a set of sub-blocks based on mutual information metrics. To obtain the correct number of information bits and block size, the encoder may remove a number of information bits equal to the number of appended additional bits (e.g., from a first half of the sub-blocks), assign the remaining information bits to bit channels in each sub-block, and block puncture a set of bits (e.g., from the first half). The resulting codeword may mitigate occurrences of achievable signal-to-noise ratio (SNR) spikes.
\end{abstract}

\section{ANNEX 10}

United States Patent Application

20190182503

Kind Code

Tsai; Yi-Ting ; et al.

METHOD AND IMAGE PROCESSING APPARATUS FOR VIDEO CODING

\section{Abstract}


A method and an image processing apparatus for video coding are proposed. The method is applicable to an image processing apparatus and includes the following steps. A current coding unit is received, and the number of control points of a current coding unit is set, where the number of control points is greater than or equal to 3. At least one affine model is generated based on the number of control points, and an affine motion vector corresponding to each of the at least one affine model is computed. A motion vector predictor of the current coding unit is computed based on the at least one motion vector so as to accordingly perform interprediction coding on the current coding unit.

\title{
ANNEX 11
}

United States Patent Application

20190190578

Kind Code

A1

Mittal; Udar ; et al.

June 20, 2019

\section{CODING MAIN BEAM INFORMATION IN CSI CODEBOOK}

\begin{abstract}
Apparatuses, methods, and systems are disclosed for preparing a channel state information ("CSI") codeword. One apparatus includes a processor and a transceiver configured to communicate 805 with a transmit-receive point ("TRP") over a radio access network using spatial multiplexing, wherein multiple transmission layers are transmitted at a time, each transmission layer comprising multiple beams. The processor identifies a main beam for each of the multiple transmission layers and determines whether the main beams of each transmission layer are the same. The processor prepares a CSI codeword, wherein the CSI codeword comprises a first bit indicating whether the main beams of each transmission layer are the same, a first set of bits coding the main beams, and a second set of bits coding the remaining beams. The transceiver transmits the CSI codeword to the TRP.
\end{abstract}

\section{ANNEX 12}

United States Patent Application

20190200025

Kind Code

A1

Li; Bin ; et al.

June 27, 2019

\section{CODED-BLOCK-FLAG CODING AND DERIVATION}

\begin{abstract}
Techniques for coding and deriving (e.g., determining) one or more coded-block-flags associated with video content are described herein. A coded-block-flag of a last node may be
\end{abstract}


determined when coded-block-flags of preceding nodes are determined to be a particular value and when a predetermined condition is satisfied. In some instances, the predetermined condition may be satisfied when log.sub.2(size of current transform unit) is less than log.sub.2 (size of maximum transform unit) or log.sub.2(size of current coding unit) is less than or equal to log.sub.2(size of maximum transform unit) +1 . The preceding nodes may be nodes that precede the last node on a particular level in a residual tree.

\title{
ANNEX 13
}

United States Patent Application

20190222863

Kind Code

HANNUKSELA; Miska Matias ; et al.

July 18, 2019

\author{
VIDEO CODING AND DECODING
}

\begin{abstract}
There is disclosed a method, an apparatus, a server, a client and a non-transitory computer readable medium comprising a computer program stored therein for multi view video coding and decoding. View random access (VRA) pictures or access units are coded into a multiview bitstream. VRA pictures enable starting the decoding of a subset of the views present in the bitstream. The views selected to be accessible in VRA pictures are alternated in successive VRA pictures so that all views are gradually reconstructed when two or more VRA pictures have been decoded.
\end{abstract}

\section{ANNEX 14}

United States Patent Application

20190200026

Kind Code

LAINEMA; Jani ; et al.

June 27, 2019

\section{APPARATUS, A METHOD AND A COMPUTER PROGRAM FOR VIDEO CODING}

\begin{abstract}
There is disclosed an apparatus, a method and a computer program for video coding. The apparatus comprises a selector configured for selecting a pixel for prediction; a projection definer configured for determining a projection of said pixel to a set of reference pixels; and a prediction definer configured for selecting one or more reference pixels from said set of reference pixels on the basis of said projection, and using said selected one or more reference pixels to obtain a prediction value for said pixel to be predicted.
\end{abstract}




\title{
ANNEX 15
}

United States Patent Application

20190208222

Kind Code

UGUR; Kemal ; et al.

July 4, 2019

APPARATUS, A METHOD AND A COMPUTER PROGRAM FOR VIDEO CODING AND DECODING

\begin{abstract}
There are disclosed various methods, apparatuses and computer program products for video encoding and decoding. In other embodiments, there is provided a method, an apparatus, a computer readable storage medium stored with code thereon for use by an apparatus, and a video encoder, for encoding a scalable bitstream, to provide indicating an encoding configuration, where only samples and syntax from intra coded pictures of base layer is used for coding the enhancement layer pictures. In other embodiments, there is provided an apparatus, a computer readable storage medium stored with code thereon for use by an apparatus, and a video decoder, for decoding a scalable bitstream, to receive indications of an encoding configuration, where only samples and syntax from intra coded pictures of base layer is used for coding the enhancement
\end{abstract}

\section{ANNEX 16}

United States Patent Application

20190222859

Kind Code

CHUANG; Tzu-Der ; et al.

July 18, 2019

\section{METHOD AND APPARATUS OF CURRENT PICTURE REFERENCING FOR VIDEO CODING}

\begin{abstract}
A method and apparatus for a video coding system with the current picture referencing (CPR) mode enabled are disclosed. According to one method, the luma and chroma blocks of the current image are jointly coded using a same coding unit (CU) structure if the CPR mode is selected for the luma and the chroma blocks. Alternatively, if the luma and chroma components are partitioned into the luma and the chroma blocks separately using separate $\mathrm{CU}$ structures, the luma and chroma blocks are encoded or decoded using a coding mode selected from a coding mode group excluding the CPR mode. According to another method, the luma and chroma blocks of the current image are coded separately using a different CU structure if the
\end{abstract}


CPR mode is selected for the luma and chroma blocks. In yet another method, reconstructed reference data is disclosed for the CPR mode with CU equal to PU.

\title{
ANNEX 17
}

United States Patent Application

20190230365

Kind Code

Tanner; Jason ; et al.

July 25, 2019

\section{VIDEO CLUSTER ENCODING FOR MULTIPLE RESOLUTIONS AND BITRATES WITH PERFORMANCE AND QUALITY ENHANCEMENTS}

\begin{abstract}
Techniques related to video cluster encoding are discussed. Such techniques include encoding the video at a first resolution and first bitrate, translating block based coding parameters corresponding thereto to block based encode controls for encode of the same video at a second resolution or a second bitrate, and encoding the video at the second resolution and/or bitrate using the encode controls.
\end{abstract}

\section{ANNEX 18}

United States Patent Application

20190246126

Kind Code

Abbas; Adeel ; et al.

August 8, 2019

APPARATUS AND METHODS FOR VIDEO COMPRESSION USING MULTIRESOLUTION SCALABLE CODING

\begin{abstract}
Apparatus and methods for digital video data compression via a scalable, multi-resolution approach. In one embodiment, the video content may be encoded using a multi-resolution and/or multi-quality scalable coding approach that reduces computational and/or energy load on a client device. In one implementation, a low fidelity image is obtained based on a first full resolution image. The low fidelity image may be encoded to obtain a low fidelity bitstream. A second full resolution image may be obtained based on the low fidelity bitstream. A portion of a difference image obtained based on the second full resolution image and the first full resolution may be encoded to obtain a high fidelity bitstream. The low fidelity bitstream and the high fidelity bitstream may be provided to e.g., a receiving device.
\end{abstract}


ANNEX 19

United States Patent Application

20190246118

Kind Code

A1

YE; Jing ; et al.

August 8, 2019

METHOD AND APPARATUS FOR VIDEO CODING IN MERGE MODE

\begin{abstract}
A method for video coding using a merge mode by a decoder or encoder. An embodiment of the method includes receiving a current block having a block size, setting a grid pattern based on the block size of the current block, wherein the grid pattern partitions a search region adjacent to the current block into search blocks, and a size of the search blocks is determined according to the block size of the current block, and searching for one or more spatial merge candidates from candidate positions in the search blocks to construct a candidate list that includes the one or more spatial merge candidates.
\end{abstract}

\title{
ANNEX 20
}

United States Patent Application

20190034583

Kind Code

Kartalov; Emil P. ; et al.

January 31, 2019

SIGNAL ENCODING AND DECODING IN MULTIPLEXED BIOCHEMICAL ASSAYS

\begin{abstract}
This disclosure provides methods, systems, compositions, and kits for the multiplexed detection of a plurality of analytes in a sample. In some examples, this disclosure provides methods, systems, compositions, and kits wherein multiple analytes may be detected in a single sample volume by acquiring a cumulative measurement or measurements of at least one quantifiable component of a signal. In some cases, additional components of a signal, or additional signals (or components thereof) are also quantified. Each signal or component of a signal may be used to construct a coding scheme which can then be used to determine the presence or absence of any analyte.
\end{abstract}

\section{ANNEX 21}

United States Patent Application

20170236521

Kind Code 


\title{
Chebiyyam; Venkata Subrahmanyam Chandra Sekhar ; et al.
}

\section{ENCODING OF MULTIPLE AUDIO SIGNALS}

\begin{abstract}
A device includes an encoder and a transmitter. The encoder is configured to determine a mismatch value indicative of an amount of temporal mismatch between a reference channel and a target channel. The encoder is also configured to determine whether to perform a first temporal-shift operation on the target channel at least based on the mismatch value and a coding mode to generate an adjusted target channel. The encoder is further configured to perform a first transform operation on the reference channel to generate a frequency-domain reference channel and perform a second transform operation on the adjusted target channel to generate a frequency-domain adjusted target channel. The encoder is also configured to estimate one or more stereo cues based on the frequency-domain reference channel and the frequency-domain adjusted target channel. The transmitter is configured to transmit the one or more stereo cues to a receiver.
\end{abstract}




\title{
Computer Implementation of the Dichotomy Algorithm for Transcendent Equations when Determining the Thread Tension
}

\author{
V. Shcherban, A. Petko, Ju. Makarenko, N. Kolva, D. Egorov \\ Kyiv National University of Technologies and Desing, Ukraine
}

\begin{abstract}
The studies of the computer implementation of the dichotomy algorithm for determining the thread tension on technological equipment determined the values of the thread tension. It is proved that the number of guides on each specific technological machine, the radius of curvature of each guide, the angle of the thread of the guide, the angle of the radial coverage of the thread, the physic-mechanical and structural characteristics of the thread influence the magnitude of the tension of the threads.

The value of the angles of the thread guides and the angles of radial coverage of the thread by the surface of the guide are determined by the geometric parameters and the design of both the filing system on the technological equipment and specific guides. Thanks to this, it became possible at the initial stage of the design of the technological process to determine the tension of the thread in front of the formation zone, depending on the geometric and structural parameters of the equipment and the physico-mechanical and structural characteristics of the thread.

The increase in thread tension occurs due to friction in the contact area with the guides. The magnitude of the friction forces depends on the material of the thread and the guide, the ratio of their geometric dimensions (the radius of the cross section of the thread and the radius of curvature of the guide in the contact zone), the actual angle of the thread of the guide and the angle of the radial coverage of the thread on the surface of the guide, the physicomechanical and structural characteristics of the thread, tension threads in front of the guide. Sequential passage of the thread along the guides, from the entrance zone to the zone of fabric formation and knitwear, leads to a stepwise increase in tension. In this case, the output tension parameter after the previous guide will be an input parameter for the subsequent guide, which allows the use of recursion in determining the tension in front of the formation zone. Keywords: dichotomy algorithm, tension, thread, recursion, guide, radius of curvature, angle of coverage.
\end{abstract}

Statement of the problem, analysis of research and publications. The work [1] is devoted to determining the tension of a thread in interaction with various guides. This study takes into account the real physical and mechanical properties of the threads, namely its bending stiffness, the nonlinear dependence of the friction forces on the normal pressure, the real angle of the thread guide and the angle of the radial surface of the thread guide surface. The effect of kinematic parameters on the value of the thread tension is considered in [2]. In [3], the influence of the thread tension on the amount of deformation of the guide surface is established. Of interest is the work [4], in which the tension is determined depending on the structural characteristics of the thread.

Statement of purpose. The study on the computer implementation of the dichotomy algorithm for determining the thread tension in interaction with the guides of technological equipment should be considered relevant.

The system of equations that describes the change in tension over the zones will have the form 


$$
\begin{aligned}
& P_{1}=P_{0} f_{0}\left(P_{0}\right), \\
& P_{2}=P_{1} f_{1}\left(P_{1}\right) \text {, } \\
& P_{i}=P_{i-1} f_{i-1}\left(P_{i-1}\right), \\
& i=1,2 \ldots n \text {, }
\end{aligned}
$$

where $P_{0}$ - thread tension in the zone of entry into the filing system of the technological machine; $P_{1}, P_{2} \ldots P_{i}$ - thread tension when leaving the corresponding zone; $f_{0}\left(P_{0}\right), f_{1}\left(P_{1}\right) \ldots f_{i-1}\left(P_{i-1}\right)$ functions that bind the thread tension before and after the guide in each zone; $i$ - current zone number; $n$ - the number of guides in the threading system of a particular technological machine.

Using a recursive approach to determining the tension of the thread in front of the fabric and knitwear formation zone, in which the output tension after the guide in the previous zone will be the input tension value in front of the guide in the subsequent zone, we will represent equations (1) in the form

$$
P_{n}=P_{0} \prod_{i=0}^{n} f_{i}\left(P_{i}\right)
$$

The fact is that the dependence that connects the thread tension before and after the guide is a system of two transcendental equations. To determine the root of the transcendental equation in the work, the dichotomy method was used.

Analysis of the conditions of interaction of a thread whose diameter is equal to $2 r$, from $i$ radius guide $R_{i}$ showed that it is necessary to consistently determine its tension in sections $0 \mathrm{~A}$, $\mathrm{AB}$ and B1. It is obvious that at points 0 (input branch that has tension $P_{i-1}$ ) and 1 (output that has tension $P_{i}$ ) the curvature of the monofilament axis is zero. The gravity of the thread can be neglected.

The general system of differential equations in projections on the axis of a natural trihedron, describing the equilibrium of an infinitesimal element of a thread, has the form

$$
\begin{aligned}
& \frac{d P}{d s}-K Q+F_{\tau}=0, \\
& \frac{d Q}{d s}+K P+F_{n}=0, \\
& \frac{d M}{d s}+Q=0, \quad M=B K, B=E I, \\
& K=\frac{1}{\rho}, \rho=\frac{d s}{d \varphi},
\end{aligned}
$$


where $P$ - thread tension;

$s$ - arc coordinate;

$K$ - thread axis curvature;

$Q$ - shear force in the cross section of the thread;

$F_{\tau}, F_{n}$ - projections of external forces on the tangent and normal axis of a natural trihedron;

$M$ - thread bending moment;

$\rho$ - the radius of curvature of the axis of the thread;

$\phi_{i}$ - angle of surface coverage between the initial and final sections of the thread. Considering that external forces do not act on monofilament in sections $0 \mathrm{~A}$ and $\mathrm{B} 1$, we can transform the system of differential equations (3) to the form

$$
\frac{d P}{d s}-K Q=0, \frac{d Q}{d s}+K P=0, \frac{d M}{d s}+Q=0 .
$$

Integration of the system of differential equations (4), taking into account the boundary conditions for points $0, \mathrm{~A}, \mathrm{~B}, 1$, made it possible to obtain the following equations for determining the tension at points $\mathrm{A}$ and $\mathrm{B}$

$$
P_{A}=P_{i-1}-\frac{B}{2\left(R_{i}+r\right)^{2}}, P_{B}=P_{i}-\frac{B}{2\left(R_{i}+r\right)^{2}} .
$$

In turn, the expressions for determining the tension force (always directed tangentially to the axis of the thread) at points A and B can be obtained by parallel transfer of the tension vectors $\vec{P}_{i-1}, \vec{P}_{i}$ at points A and B and decomposing them into tangent and normal using stiffness angles $\gamma_{0 i}, \gamma_{1 i}$. Then we get

$$
P_{A}=P_{0} \cos \left(\gamma_{0 i}\right), P_{B}=P_{1} \cos \left(\gamma_{1 i}\right) .
$$

Solving equations (5) and (6) together, we obtain expressions for determining the stiffness angles

$$
\gamma_{0 i}=\arccos \left[1-\frac{B}{2 P_{i-1}\left(R_{i}+r\right)^{2}}\right], \gamma_{1 i}=\arccos \left[1-\frac{B}{2 P_{i}\left(R_{i}+r\right)^{2}}\right] .
$$

Thus, the actual angle of the thread, taking into account (8), can be determined from the expression

$$
\phi_{i}=\phi_{P i}-\arccos \left[1-\frac{B}{2 P_{i-1}\left(R_{i}+r\right)^{2}}\right]-\arccos \left[1-\frac{B}{2 P_{i}\left(R_{i}+r\right)^{2}}\right],
$$

where $\phi_{P i}$ - estimated value of the angle of coverage $i$ guide.

We proceed to the determination of the tension of the thread in the section $\mathrm{AB}$. In this section, external friction forces will act on the thread $F$ and normal pressure $N$. We assume that the 
friction force is proportional to normal pressure, and the variable is the value of the coefficient of friction, which depends on tension, the radius of curvature of the guide, the angle of the thread of the guide. Based on this, the friction force, taking into account the radial coverage of the thread by the surface of the guide, can be represented as

$$
F=\mu N, \mu=a \frac{4 \sin \left(\frac{\beta_{i}}{2}\right)}{\beta_{i}+\sin \left(\beta_{i}\right)}\left(\frac{P}{R_{i}}\right)^{n_{1}} .
$$

Consider the equilibrium of the element of the thread in section AB. Given that the radius of curvature of the cylinder $R_{i}$ is a transverse force constant $Q=O$. Then, taking into account (10), the system of differential equations (3) takes the form

$$
\begin{aligned}
& \frac{d P}{d s_{i}}-a \frac{4 \sin \left(\frac{\beta_{i}}{2}\right)}{\beta_{i}+\sin \left(\beta_{i}\right)}\left(\frac{P}{R_{i}}\right)^{n_{1}} N=0, \\
& \frac{P}{R_{i}}-N=0, d s_{i}=R_{i} \phi_{i} .
\end{aligned}
$$

We integrate the last differential equation, taking into account the boundary conditions at points $\mathrm{A}$ and $\mathrm{B}$, we obtain

$$
P_{B}^{-n_{1}}-P_{A}^{-n_{1}}=-\frac{4 \sin \left(\frac{\beta_{i}}{2}\right)}{\beta_{i}+\sin \left(\beta_{i}\right)} \frac{n_{1} a \phi_{i}}{R_{i}^{n_{1}}}
$$

We transform equation (12) using the Lopital rule and find the limit for $n_{1} \rightarrow 0$

$$
P_{B}=P_{A} e^{a \frac{4 \sin \left(\frac{\beta_{i}}{2}\right)}{\beta_{i}+\sin \left(\beta_{i}\right)}\left(\frac{P_{A}}{R_{i}}\right)^{n_{1}} \phi_{i}} .
$$

Solving together (5), (8), (12), we obtain a system of two transcendental equations for the tension of the leading branch of the monofilament $P_{1}$

$$
\begin{aligned}
& P_{i}=P_{i-1} e^{a\left[\frac{P_{i-1}-\frac{B}{2\left(R_{i}+r\right)^{2}}}{R_{i}}\right]^{n_{1}} \phi_{i}}+\frac{B}{2\left(R_{i}+r\right)^{2}}\left\{1-e^{a\left[\frac{P_{i-1}-\frac{B}{2\left(R_{i}+r\right)^{2}}}{R_{i}}\right]^{n_{1}} \phi_{i}}\right\}, \\
& \phi_{i}=\phi_{P i}-\arccos \left[1-\frac{B}{2 P_{i-1}\left(R_{i}+r\right)^{2}}\right]-\arccos \left[1-\frac{B}{2 P_{i}\left(R_{i}+r\right)^{2}}\right] \text {. }
\end{aligned}
$$

Then the connection function of the thread tension before and after the guide will be

$$
f_{i-1}\left(P_{i-1}\right)=e^{a\left[\frac{P_{i-1}-\frac{B}{2\left(R_{i}+r\right)^{2}}}{R_{i}}\right]^{n_{1}} \phi_{i}}+\frac{B}{2 P_{i-1}\left(R_{i}+r\right)^{2}}\left\{1-e^{a\left[\frac{P_{i-1}-\frac{B}{2\left(R_{i}+r\right)^{2}}}{R_{i}}\right]^{n_{1}} \phi_{i}}\right\} .
$$


Figure 1 shows the main forms of the program for determining the tension. Figure 1a shows the results of determining the tension for a nylon complex thread with a design diameter of 0.2 $\mathrm{mm}$. Figure $1 \mathrm{~b}$ shows the results of determining the tension for a nylon monofilament $0.2 \mathrm{~mm}$ in diameter.

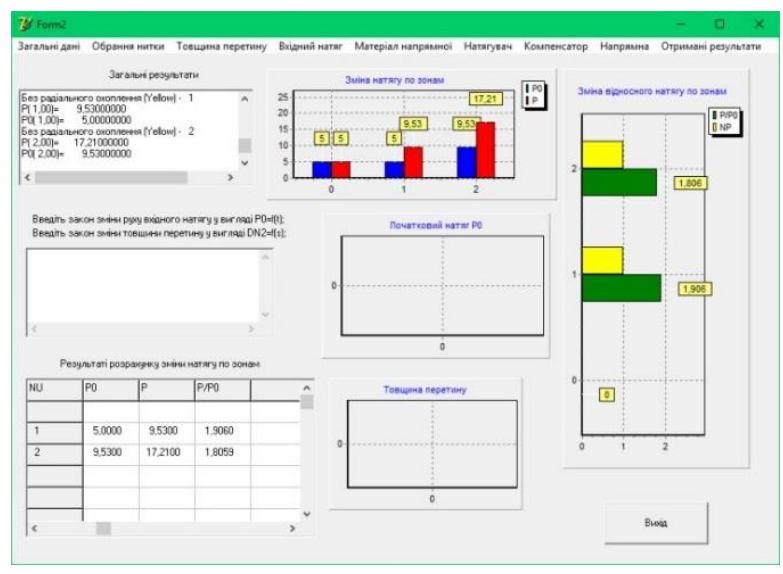

a

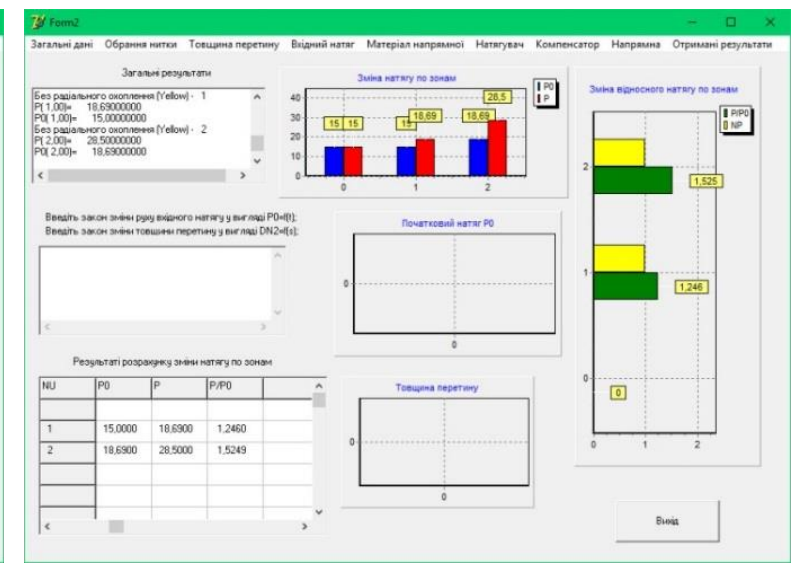

b

Picture 1

Conclusions. Based on the developed block diagram, a computer implementation of the dichotomy algorithm was carried out when determining the tension of the thread in interaction with the guides of the technological equipment. Studies have shown that the number of guides on each specific technological machine affects the tension of the filament in front of the formation zone.

Based on the developed theoretical model, a computational experiment was carried out to determine the tension and the function of linking the tension of the thread before and after the guide, taking into account the radius of curvature of the guide, the angle of the thread of the guide, the angle of the radial coverage of the thread, physical and mechanical and structural characteristics of the thread. It has been established that the value of the angles of filament guides and the angles of radial envelopment of the filament by the surface of the guide are determined by the geometric parameters and design of both the filing system on the technological equipment and specific guides.

\section{References}

1. Scherban V. Interaction yarn guide surface/V.Scerban, M. Sholudko, V. Kalashnik, O. Kolisko//Intellectual Archive, Toronto: Shiny World Corp., Richmond Hill, Ontario, Canada. May 2015. - Volume 4.- Number 3. - P. 10-15.

2. Scherban V. Kinematics of threads cooperates with the guiding surfaces of arbitrary profile /V. Scherban, N. Murza, O. Kolisko, M. Sheludko, I. Semenova //Intellectual Archive, Toronto: Shiny World Corp., Richmond Hill, Ontario, Canada. - May/June - 2016. - Volume 5.- Number 3. - P. 23-27. 
3. Scherban V. Basic parameters of curvature and torsion of the deformable thread in contact with runner/V. Scherban, N.Murza, A.Kirichenko, O. Kolisko, M. Sholudko //Intellectual Archive, Toronto: Shiny World Corp., Richmond Hill, Ontario, Canada. - Nov/Dec - 2016. Volume 5.- Number 6. - P. 18-23.

4. Scherban V. Equalizations of dynamics of filament interactive with surface/V. Scherban, G. Melnik, A.Kirichenko, O. Kolisko, M. Sheludko//Intellectual Archive, Toronto: Shiny World Corp., Richmond Hill, Ontario, Canada. - January/February 2017. - Volume 6.- Number 1. P. 22-26. 
DOI 10.32370/IA_2020_01_8

\title{
Modern Methods of Processing and Utilization of Oil Sludge
}

\author{
Saidov Jafar Jamshedovich \\ Student of Kazan National \\ Research Technological University, Uzbekistan, Bukhara.
}

\begin{abstract}
Hazardous pollutants of all natural environmental components are oil-containing waste, that are oil sludge. Storage of oil sludge causes complex ecologic problems, while at the same time, its oily component is valuable organic raw material. In this article it's considered the problem of eliminating environmental pollution by oil-containing waste and there are provided modern methods of utilization and processing of oil sludge. There are set comparative description of main pros and cons, effectiveness of disposal methods.
\end{abstract}

Keywords: ecology, oil sludge, pollution sources, utilization methods, best available techniques.

\section{Introduction:}

Oil producing and oil refining industries currently play a huge role in economy of our country. But unfortunately, oil industry in terms of negative impact on environment occupies one of the first places in the national economy sectors.

\section{Relevance:}

Significance of problem is determined by both considerable amount, and negative impact of oil waste on almost all environmental components. One of the tasks aimed at reducing negative impact of waste on the environment and improving resource supply of economy is disposal of oil-containing waste. One of the most dangerous pollutants of almost all components of environment, i.e. surface and groundwater, soil cover, atmospheric air is oilcontaining waste that is oil sludge.

\section{Goals:}

Analyze the main advantages and disadvantages of modern methods of oil sludge utilization and processing.

Oil sludge is a complex physic-chemical mixture, which consists of oil products, mechanical impurities (clay, metal oxides, sand) and water. The ratio of its component 
elements can differ at all. Qualitative characteristics of oil sludge at enterprises fall within the following limits: ${ }^{1}$

- organic substances from 10 up to 25 percent by mass;

- mechanical impurities from 5 up to 30 percent by mass;

- water from 50 up to 70 percent by mass.

Basic methods of utilization and neutralization of hydrocarbon-containing wastes are: thermal, chemical, physical, physicochemical and biological. ${ }^{2}$ Choice of a method for processing and neutralization of oil sludge mainly depends on amount of oil products in them: in each case, when choosing an option for neutralization and purification of oil sludge at enterprises, it's necessary differentiated approach taking into account both environmental and economic indicators.

Thermal methods. Main thermal method of disposal is burning of oil sludge in chamber, bubbler, pit, fluidized bed and rotary furnaces. Process requirements: $\mathrm{t}=800-1200$ ${ }^{\circ} \mathrm{C}$, excess of oxygen.

\footnotetext{
${ }^{1}$ Mustafin I.A., Akhmetov A.F., Gaysina A.R. Methods of utilization of oil sludge of various origin // Oil and gas business. 2011. T.9, №3.

${ }^{2}$ Zhumaev K.K., Oripova L.N. Choice of method for neutralization and purification of oil sludge // Young scientist. 2014. № 1 (60).
} 


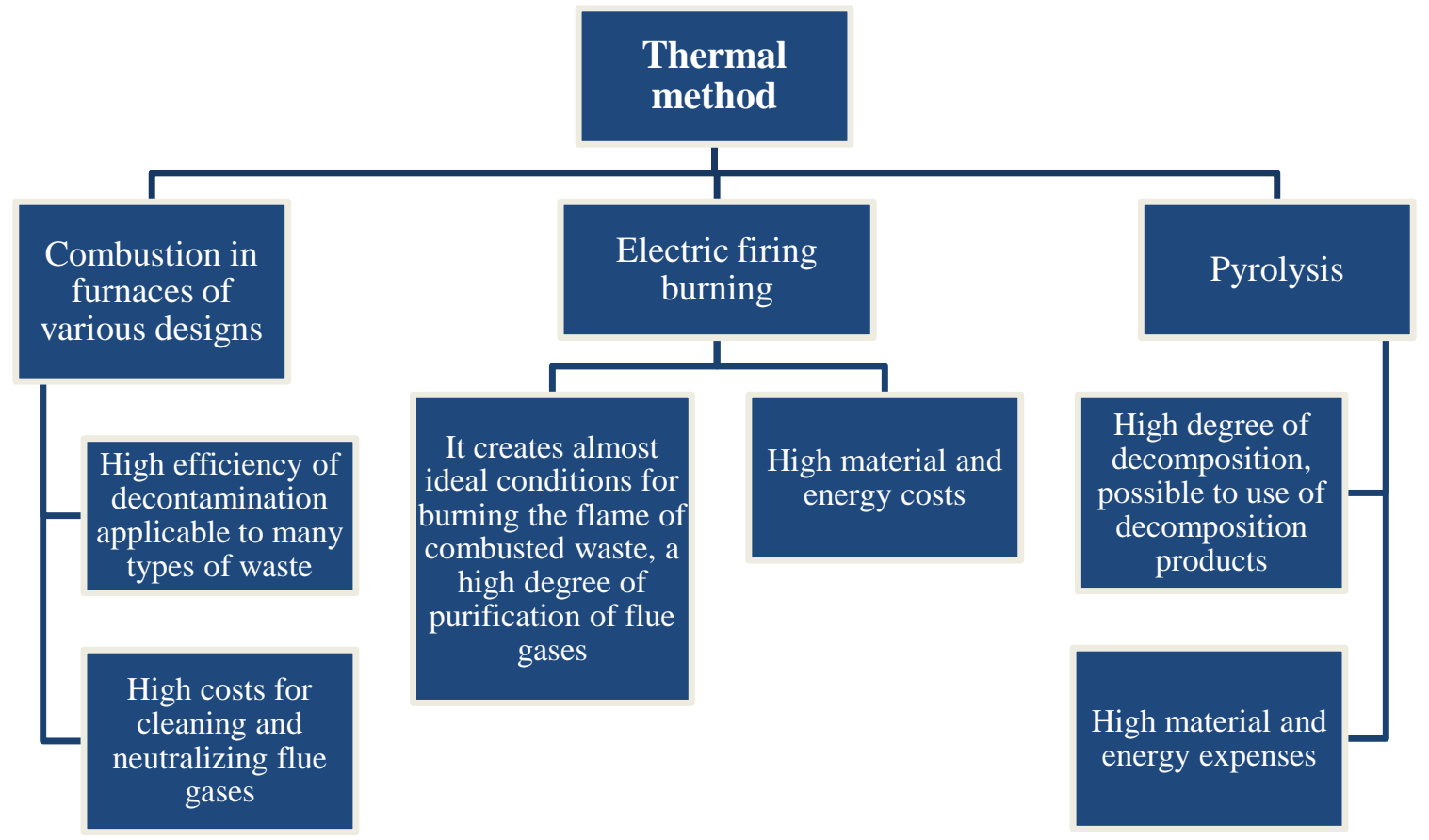

Chemical methods. Chemical methods of utilization of oil sludge are characterized by use of solvent. As solvents for dispersing oil sludge, there are used low-boiling paraffin hydrocarbons, for example, n-hexane, a wide fraction of light hydrocarbons, gas condensate, etc. One of the most used reagents in the practice of oil sludge disposal by chemical method is calcium oxide, or quicklime. The most common method of chemical neutralization is processing of quicklime waste in amount of 5-50\% by weight (amount of lime is determined by results of chemical analysis of oil sludge), pre-treated with stearic acid or other surfactants. After drying in natural conditions during 2-20 days, it can be obtained dry, highly hydrophobic powder, which is used as a construction material in highway engineering. Oil sludge particles are enclosed in the capsule's calcareous shell and are evenly distributed throughout the product. Oil products bound by reagent thus become inert, are not washed out by water, and accordingly do not affect soil and groundwater. 


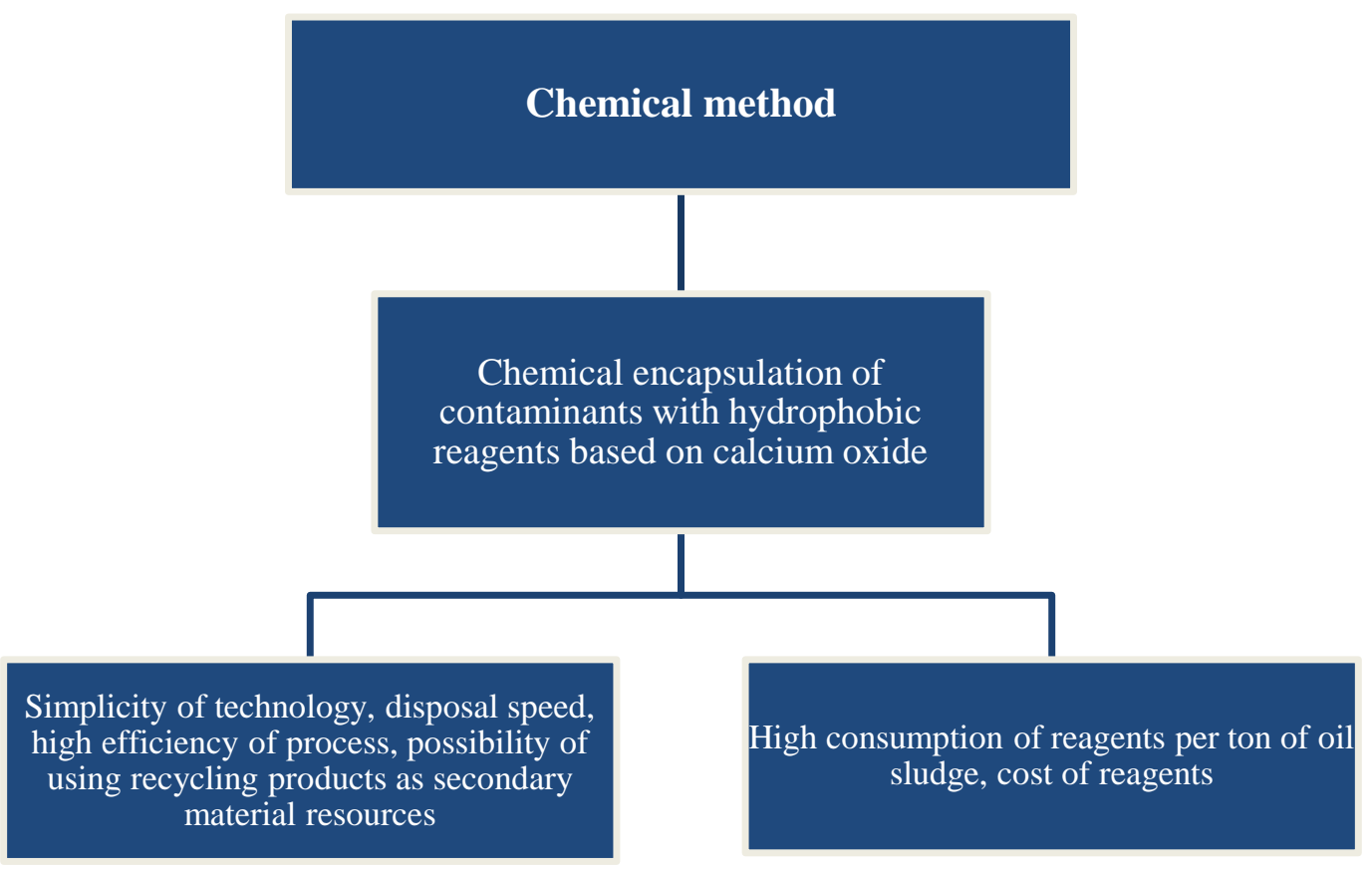

Physical methods. Physical methods of processing and utilization of hydrocarboncontaining waste are represented by hydromechanical processes. Basis of hydromechanical processes is hydrostatic or hydromechanical impact on the processed environment and materials. Such processes include stirring, settling (sedimentation), filtering, centrifugation, which have driving force like hydrostatic pressure or centrifugal force. 


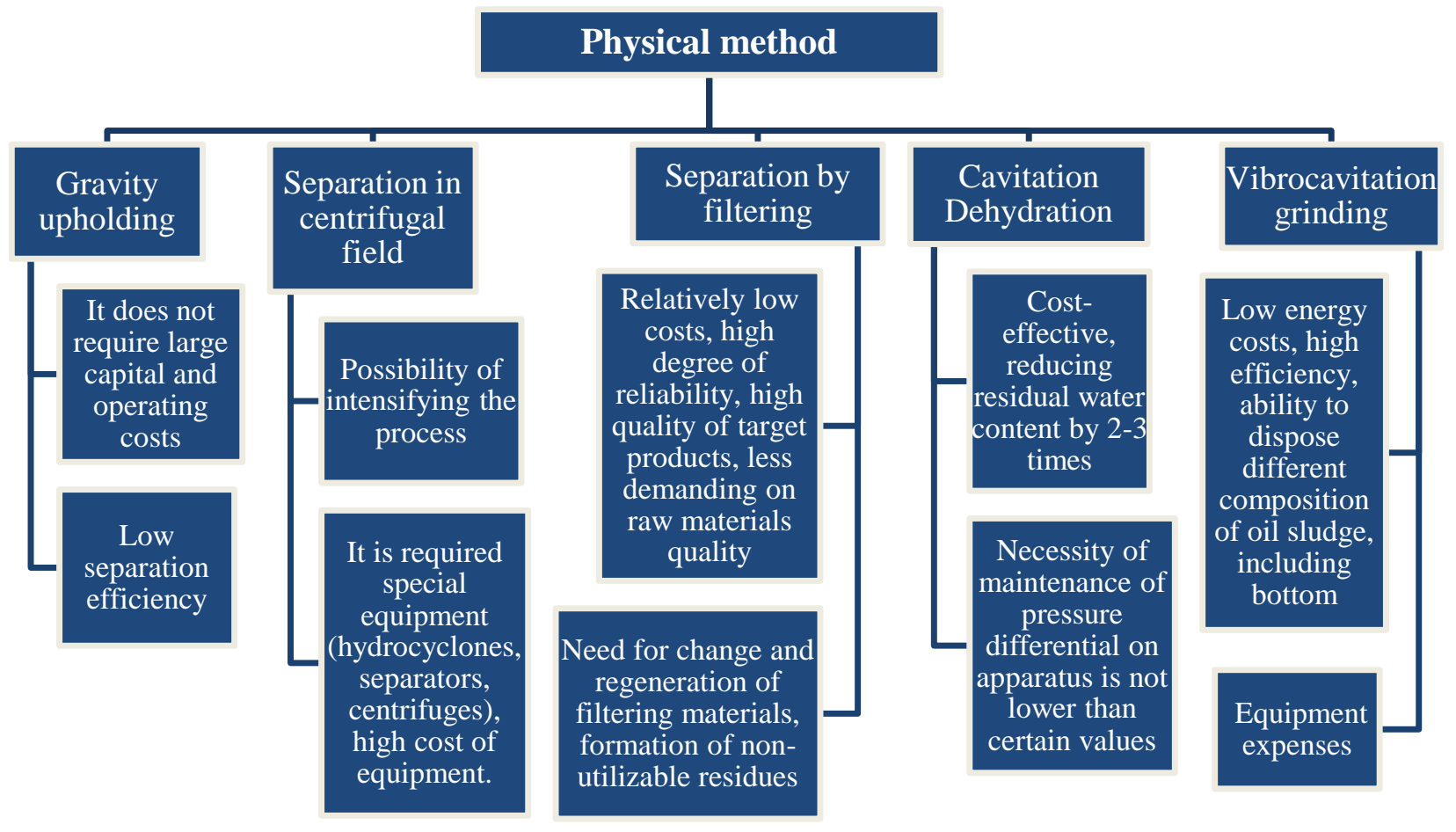

Physicochemical methods. Physicochemical methods are mostly used not only for processing and disposal, also for neutralization of hydrocarbon waste. Significant influence on change in system properties during physical and chemical processes is exerted by external conditions (pressure, volume, temperature, etc.) where they are realized. In this case, surface and interfacial properties can significantly change, and other phenomena of mixed nature will develop. Typically, oil sludge is preheated during processing, separated into their component parts: oil, water and solids, and each component will be disposed. When separated into oil and water phases, oil sludge is treated with demulsifier. 


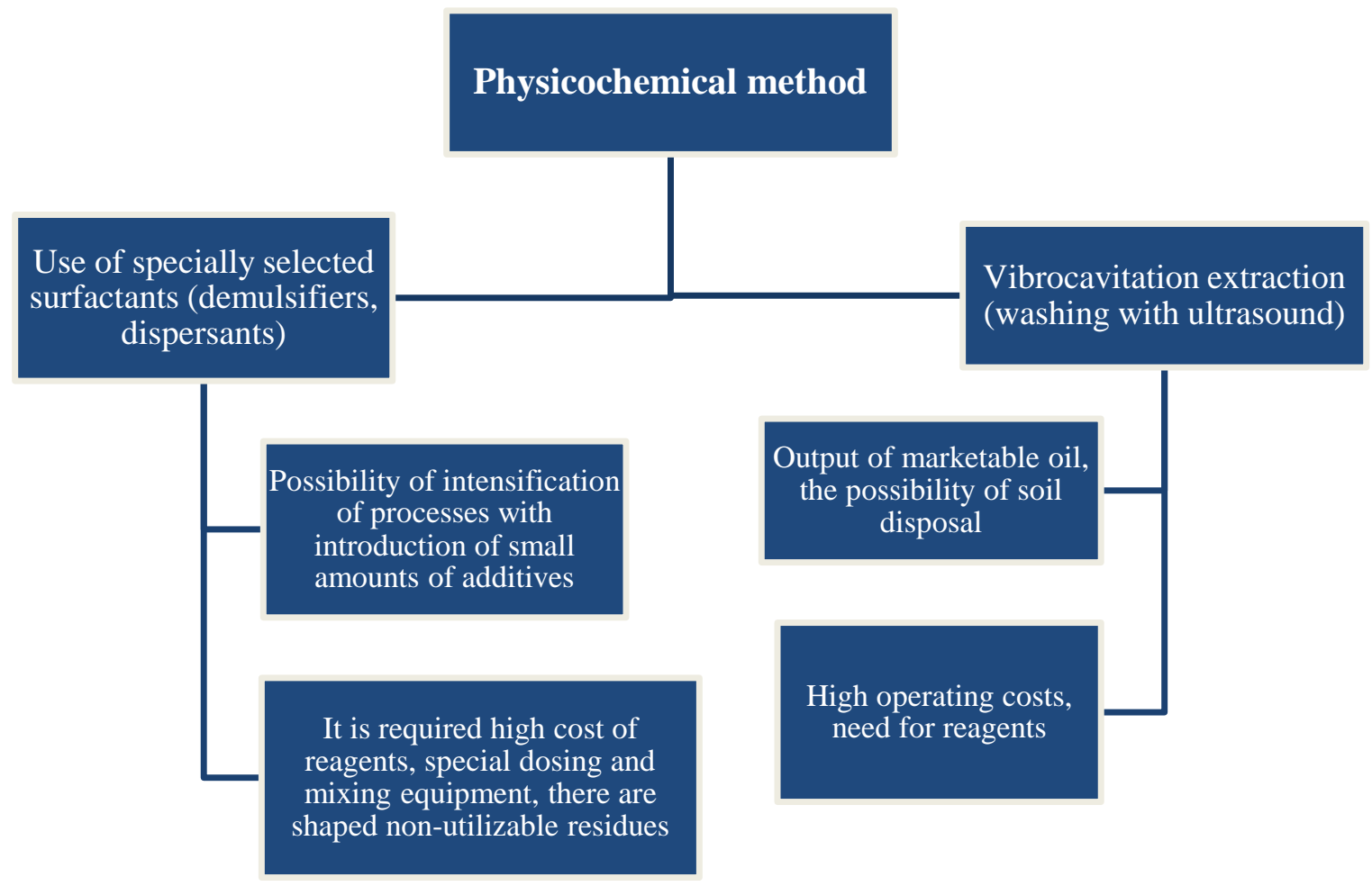

Biological methods. Biochemical methods for decontamination of hydrocarboncontaining waste are increasingly used in our country and especially abroad. Biochemical processes are chemical transformations that occur with participation of wildlife, acting as biological catalyst. Biochemical treatment of hydrocarbon-containing waste is based on ability of some microorganisms to convert aromatic and aliphatic hydrocarbons into harmless carbon dioxide and water. These reactions occur under aerobic conditions. 


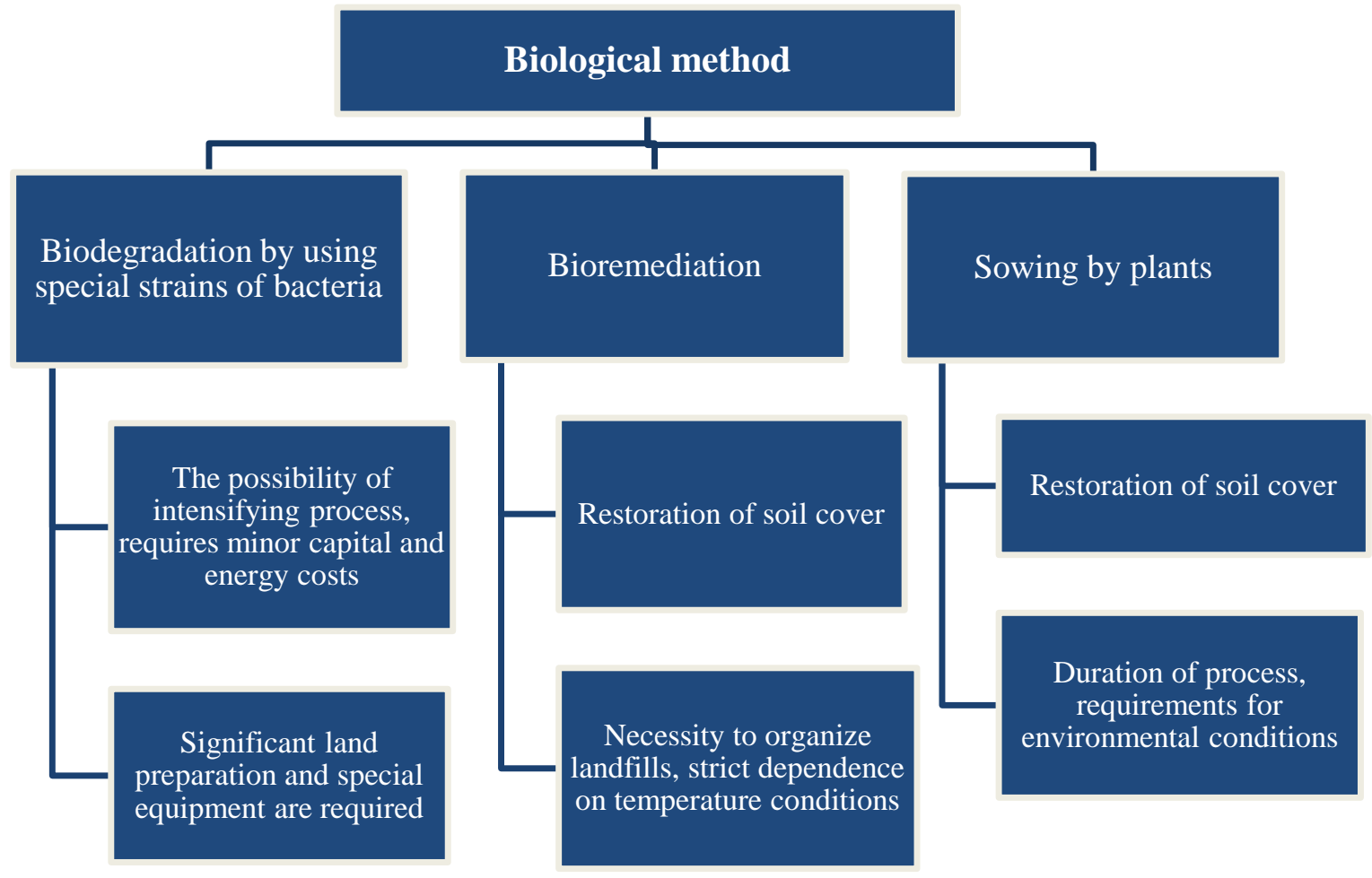

\section{Conclusion.}

Thus, it should be noted that in each case, when choosing the option of neutralizing and cleaning oil sludge for enterprises, it is necessary differentiated approach taking into account both environmental and economic indicators. Inclusion of devoted to waste disposal problems, in particular, disposal of oil sludge in targeted ecologic programs should be the priority task.

\section{References}

1. Mustafin I.A., Akhmetov A.F., Gaysina A.R. Methods of utilization of oil sludge of various origin // Oil and gas business. 2011. T.9, №3.

2. Zhumaev K.K., Oripova L.N. Choice of method for neutralization and purification of oil sludge // Young scientist. 2014. № 1 (60).

3. Mazlova E.A., Meshcheryakov S.V. Problem of oil sludge utilization and methods of their processing. 2001. 
DOI 10.32370/IA_2020_01_9

\section{Forensic Examination of Packaging with Indications of Counterfeiting Present on Optical Media in Civil and Criminal Proceedings}

Kofanov Andrii

Legal Advisor to the Council of Europe Programme "Decentralization and Territorial Consolidation in Ukraine”, PhD of Juridical Sciences, Associate

Professor, Professor of Department of Forensic Support and Forensic Expertise of the National Academy of Internal Affairs, Kiev, Ukraine ORCID ID 0000-0002-5242-2518 kofanov_andrey@ukr.net

\section{Pavlovska Nataliia}

PhD of Juridical Sciences, Associate Professor, Professor of Department of Civil Law and Process of the National Academy of Internal Affairs, Kiev, Ukraine ORCID ID 0000-0003-3311-0364_wwwpav@gmail.com

\section{Svoboda Eugenia}

PhD of Juridical Sciences, Associate Professor, Professor of Department of Forensic Support and Forensic Expertise of the National Academy of Internal Affairs, Kiev, Ukraine ORCID ID 0000-0002-8639-8333 jeechka@ukr.net

\section{Tereshchenko Yuliia}

PhD of Juridical Sciences, Professor of Criminal Law Disciplines and Operative and Investigative Activities of the Precarpathian Department of the

National Academy of Internal Affairs, Kyiv, Ukraine ORCID ID 0000-0002-5353-0887 vladysikter@ukr.net

\section{Strilets Halyna}

PhD of Judicial Sciences, Associate Professor of the Departament of Law of Prydunai Branch of Private Jointstock Company «Higher Educational Institution of Interregional Academy of Personnel Management», Izmail, Ukraine

ORCID ID 0000-0002-1067-0820 galinastrelets2018@gmail.com

\section{Abstract}

Today, forensic examination of audiovisual products distributed on optical media requires extensive use of the concepts and principles of copyright and related rights, technologies of media (incl. audio and video 
cassettes, CDs) manufacture, modern cyber techniques and devices, basic principles of the forensic identification, diagnostics and situology theories.

Keywords: expertise of copyright objects and related rights, articles, computer programs and databases, implementation of phonograms, videos, programs of loans organizations.

Introduction For forensic examination, audiovisual products represent a complicated subject. During their analysis, special knowledge from the following forensic branches is applied, depending on the problem to be addressed:

- examination of intellectual property items (to identify and decrypt notation on discs and/or cassettes, which describes holders of rights to the works, as well as the information that evidences the transfer of copyright and/or related rights; to identify the work and/or advertising unit; to identify features of counterfeit products);

- trace evidence examination (to determine the methods used in manufacturing audioand videocassettes or CDs and their packaging; to identify equipment used in the manufacture of these items, etc.);

- phonoscopy (to investigate physical parameters of an audio or video recording, identify indications of editing, identify a person by his/her voice, identify the recording equipment, etc.);

- technical analysis of documents (to study the process and quality of marking made on the surface of a disk, cassette and/or their packaging, printing materials, methods of manufacturing the Ukrainian control stamp; to identify actors in video recordings, etc.);

- technical computer analysis (to search for and identify video and audio recordings on a $\mathrm{CD}$ and in the computer's memory, to determine the type of the equipment used in replicating the work, etc.);

- chemical and/or biological examination (to study materials from which audio or videocassettes, CDs, their packaging, inserts, Ukrainian control stamps, etc., were manufactured).

Counterfeit audiovisual products have tangible features that distinguish them from the licensed ones. These features are identified through expert analysis. Their presence mostly evidences the non-compliance of a specific copy of an audiovisual product comprising a disc, packaging, layout design, control stamp, etc., with the manufacturing technology and retail trade rules that are established for a similar licenced sample copy [1]. 
As a result of rapid advances in technology and changes in the legal regulation of the manufacture and distribution of phonographic and audiovisual products, changes occur in their features; thus, a standard specimen of a sample copy used for comparison purposes also changes over time. Therefore, using a standard specimen of a licenced item is only feasible when the researcher is aware of the current state of science and technology in the field of phonographic and audiovisual products.

Upon integration of expert practice, standard specimens of licenced and counterfeit sample copies of audio products were created, having specific sets of features.

Thus, when examining external packaging of a sample phonogram copy, which may be in the form of a transparent colourless polymer film and a hard plastic or cardboard case, attention should be paid to the following signs of counterfeiting: no individual shrinkwrapping; plastic self-adhesive bags that may be re-sealed; no polymer case, or injection moulding defects of polymer case in the form of air pockets or flow lines; traces of cutting tools (scissors, etc.) on the edges of liner notes or tray card; chipping of, and small cracks around the holes, etc [2].

The layout design of discs and inlays is examined to detect the presence of the following signs: poor layout design of discs and inlays (blurred images, ghosting, distorted colour rendition of images, moiré, dots instead of uniform tint, grey scale imbalance, etc.); low-quality paper used; black and white print or no accompanying illustrated booklet; unintelligible printed (especially small) text; use of fonts other than those in licenced sample copies; grammar and spelling errors; information (usually on a label side) that the examined disc is a recordable disk; no Ukrainian control stamp, unless otherwise provided by Article 8.5 of the Law of Ukraine "On Distribution of Copies of Audiovisual Works, Phonograms, Videograms, Computer Software, Databases"; an entirely new layout design of the inlay, based on different or on somewhat modified segments (where a sample copy of the legitimate product is available); inlays printed on one side only; irregular inlay edges and folding; no copyright or related rights protection signs on the inlay or information about the phonogram author(s), manufacturer, transfer of copyright and related rights to third parties; about rightsholders within Ukraine; track titles and description; the manufacturer's addresses and/or telephone numbers for filing complaints; no information about songwriters, performers, designers, photographers, etc.; no distributor logo; no tracklist indicating duration of tracks; no album 
lyrics; no album number from the copyright holder's catalogue; no manufacturer's barcode [3].

The inlay contains false information about rightsholders, such as Meccophone 200; Navigator; Neformal LLC; Studio Retro; TUDA Records; 21 st Century G.V.D. Rus; Balalayka Records; Classic Records; Gold Records; HIGH END Records; Monsters Of Rock; Pop SHOW; Pull Dance; R-Proekt; Russian Dance Studio; Star Records; Tornado Records; Ultra Studio.

- the inlay contains information about pirate trademarks (Fig. 1) [6,7,8]:

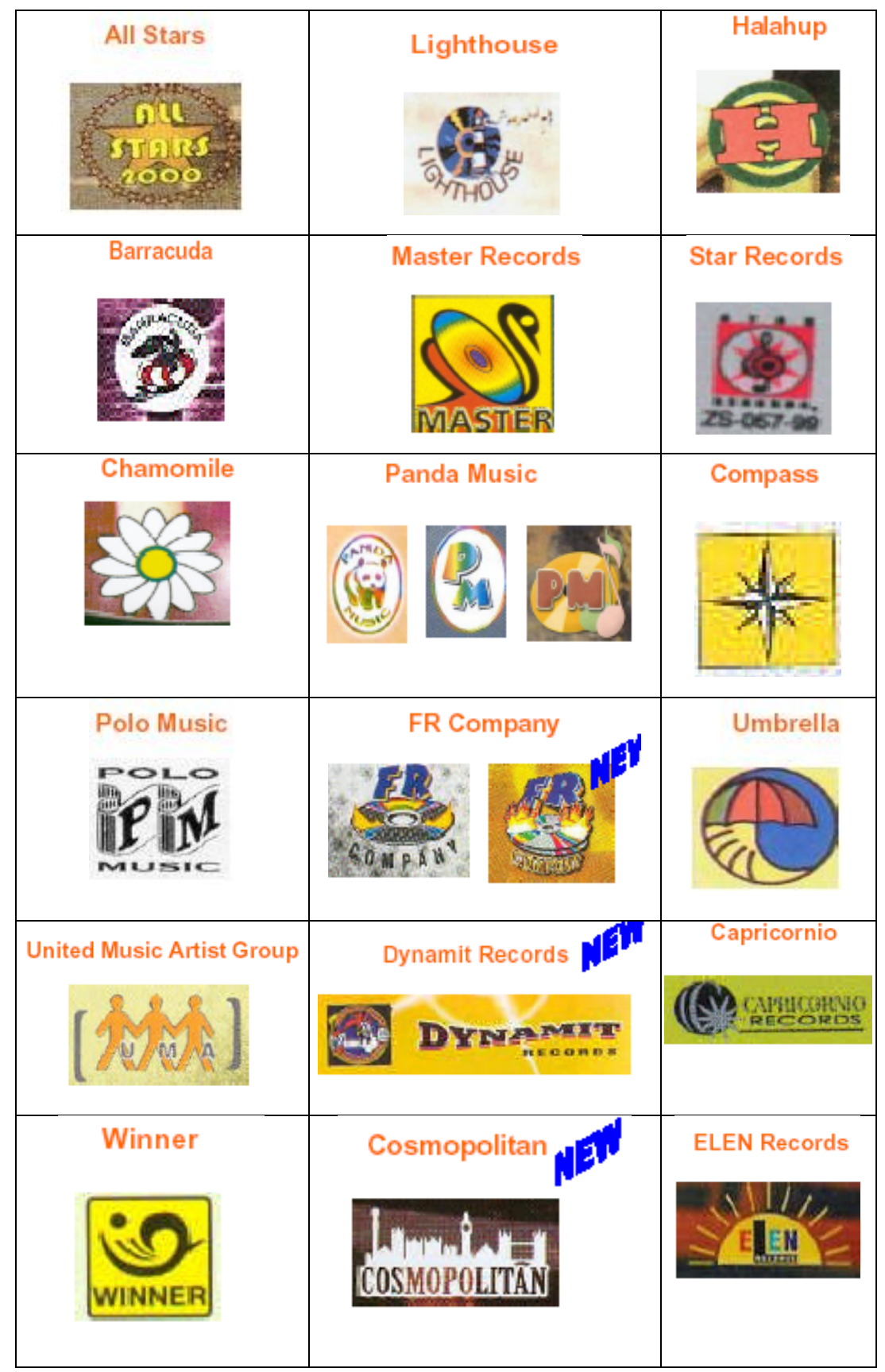




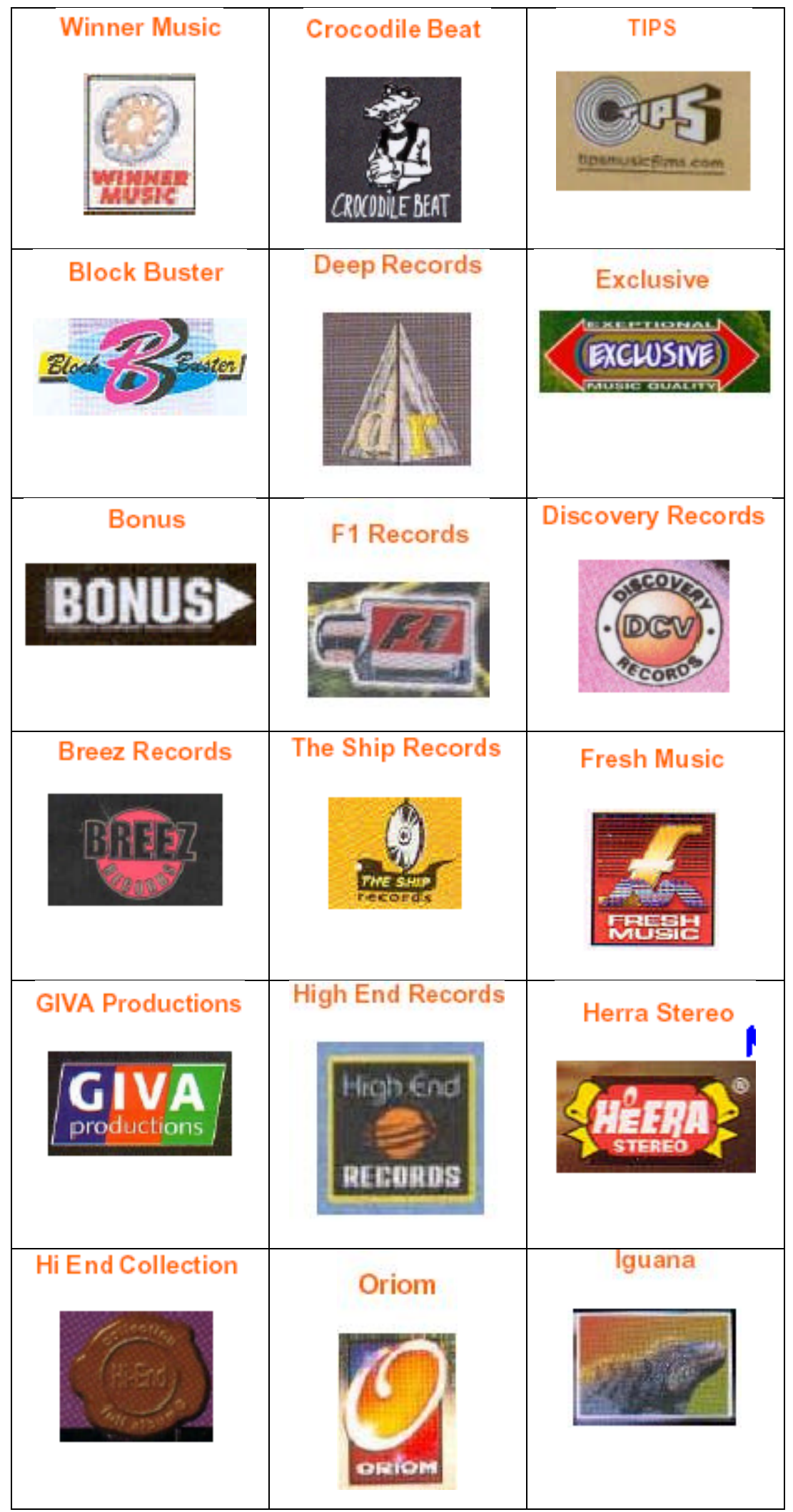




JS Records LMR Music all Soundboard Quality

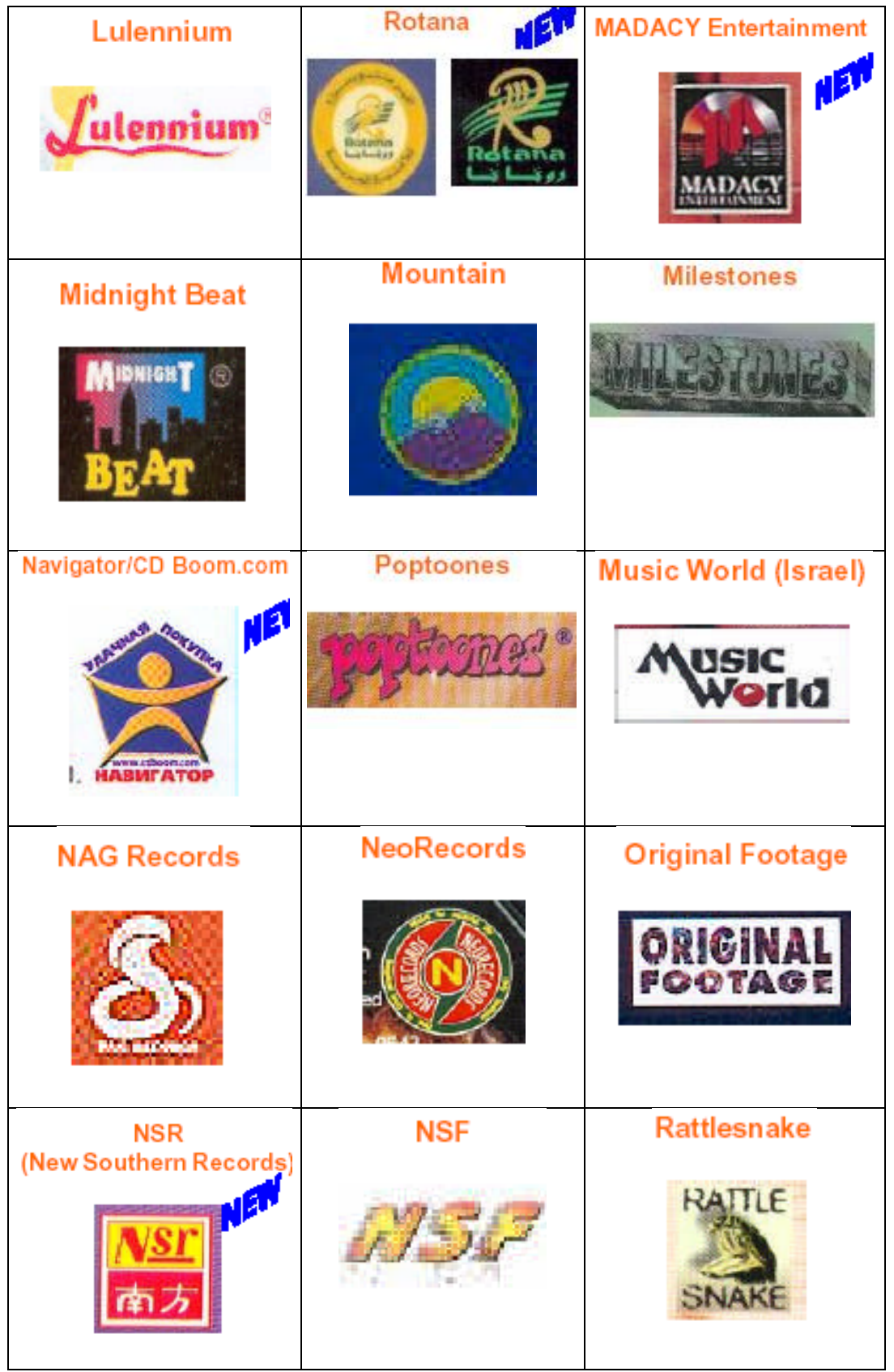




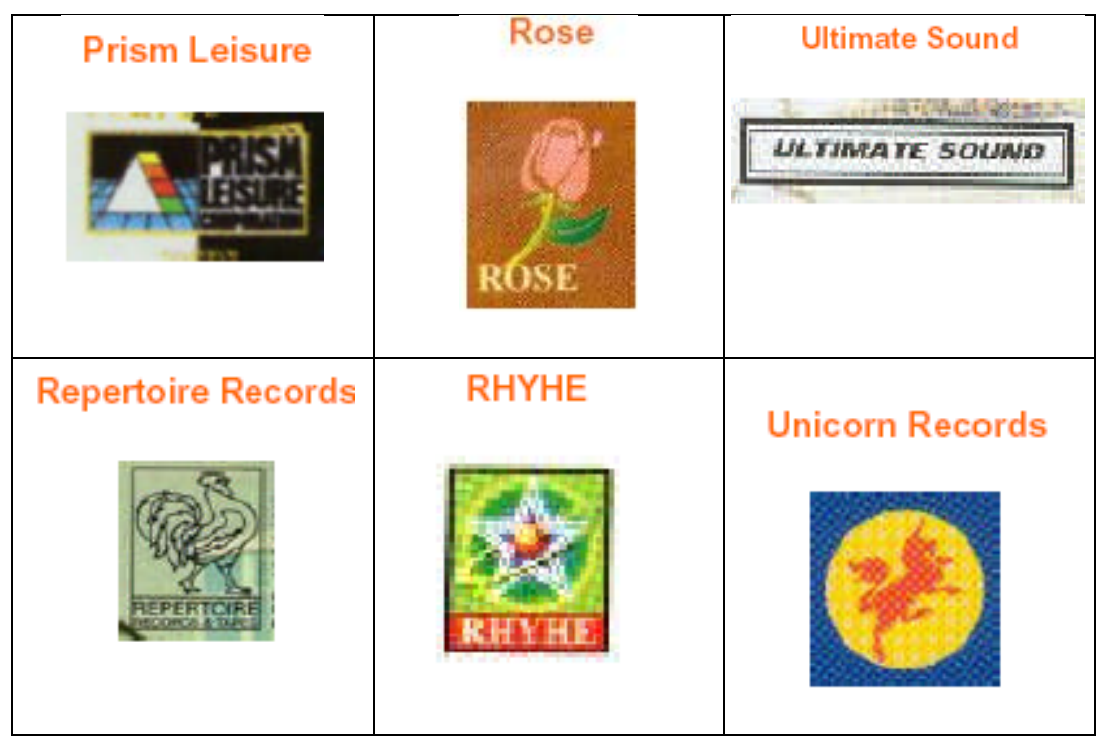

At the next stage, the storage medium - the optical disk, i.e., its label and data surfaces - is examined.

The label side of legitimate CDs contains high-quality colour images and may include information about the performer(s); track titles and description; copyright/related rights signs; first published date; information about rightsholders; rightsholder companies' logos; information about rightsholders within Ukraine; about transfer of copyright and related rights to third parties; the manufacturer's address for filing complaints.

Counterfeit CDs exhibit the lack of proper artistic design, manifested in the blurred images, misalignment of text relative to the central hole of the disc, chipped and uneven CD printing ink; discrepancy between the information provided on the CD label and in the inlay; grammar errors in the text; no names of rightsholder companies or their logos; no artist name and album title, album number under the rightsholder's catalogue.

Examining images on the audiovisual products' layout design items.

Comparative analysis of layout design between the counterfeit and legitimately manufactured products may indicate differences in a number of features.

1. Manufacturers use specific design for their legitimate products. Sometimes an entirely new design is developed for counterfeit products, using different or somewhat modified segments from the original products. If any discrepancy is identified in the design of a sample copy of the recorded audio product, a violation of the copyright to the layout design has taken place.

2. The following information is printed on the packaging: authors of audiovisual 
products; manufacturer; copyright mark (must comprise three components: the $\mathbb{C}$ copyright symbol itself, the name of the copyright holder and the date when the product was manufactured for the first time, or another protection mark for exclusive related rights "(T)", which also comprises three elements similar to those mentioned above); transfer of copyright and related rights to third parties; copyright holder; title and description of the audiovisual product; age restrictions; for erotic products - the information on a special expert examination; a brief overview of the product; details of the copyright holder (or seller) for filing complaints.

3. During the exclusive manufacture of audiovisual products, certain manufacturers use only a specific type of magnetic media whose playing time is usually different from a standard one (195 or 240 minutes). Only one film and a commercial are recorded on a videocassette.

4. Discrepancies in the design of the case, depending on the videocassette (either through the top or bottom side, or along the spine), as well as a different opening. There is no need to completely unglue the case - merely folding it will make the differences in the shape of the flaps quite obvious.

5. Using different translation for the same audiovisual product title.

6. Discolouration. Pink and red colours often tend to acquire a purple tint. This defect in graphics is particularly evident for the human eye.

7. Quality of printed images. Both vector (mostly small type and logos) and bitmap (for other images) graphics are used in the legitimately manufactured products. Cases for counterfeit products are printed using only bitmap graphics, which significantly affects the quality of smaller images. If a legitimately manufactured case was used as an original copy to print the case, superimposition of two bitmap graphics results in even more deteriorated image quality.

8. Using lower resolution bitmaps than for the original casing. This primarily affects the quality of smaller images, for example, frames from audiovisual products.

9. Fonts of other size and typeface are used. This may indicate that the images had been scanned and the texts had been retyped in a text editor.

10. Holographic stickers on the videocassette and/or its case. The hologram must contain the manufacturer's or the rightsholder's logo.

11. The use of finishing processes. In the printing industry, varnishing of the finished 
product is common. An additional section for applying varnish is often installed on printing equipment. The boundary of the varnish layer is sometimes clearly visible on the case flaps and can be detected by mechanical action: in the microscope, a transparent substance seems to appear on the surface - the so-called "dew point effect". Varnishing is intended to protect the cardboard case against soiling of the packaging. Colourless stamping with coloured tin foil is also used.

12. A sticker containing almost all the information listed in paragraph 1 must be attached to the videocassette case.

Whenever such expert examinations are performed, we generally have to handle a significant number of variously titled items of counterfeit audiovisual products. A comparison study, conducted in the manner adopted for forensic proceedings, along with a detailed description of variations from the sample copy, is quite a lengthy and time-consuming process. To make things easier, the above signs of counterfeit products are summarised in the table below [4].

Diagnostic and identification tasks are primarily addressed during the forensic analysis of chemicals (or their mixtures). To this end, a set of physical and chemical techniques for analysing their composition and production processes has been developed.

Regarding application of control stamps to copies of audiovisual works, phonograms, videograms, computer software, databases.

It should be noted that the Law "On Distribution of Copies of Audiovisual Works, Phonograms, Videograms, Computer Software, Databases" (the "Law on Control Stamps") is currently in force in Ukraine.

In practice, certain challenges, associated with the implementation of its provisions, emerged and, as a result, the control stamp mechanism has by now lost its relevance and efficiency. Shadow schemes of illegal purchase of control stamps, including through the use of bogus licence agreements, were becoming widespread. The "control stamp" protection element turned out to be faulty, allowing for initial markings to be tampered with by the perpetrators and for the counterfeit products to be stamped with such holographic elements.

The dynamics of control stamp purchases by bona fide manufacturers and distributors of copies of audiovisual works, phonograms, videograms, computer software, database 
indicate a significant decrease in the demand for this administrative service: from 14,000,000 control stamps in 2011 to $1,100,000$ in 2017.

In particular, national associations representing the interests of the music industry challenge further application of control stamps to phonogram copies [5].

A similar point of view has been voiced by international organisations for quite a long time. For example, in its 2010 Report, the International Intellectual Property Alliance (IIPA) noted that the existing hologram system in Ukraine is regarded by foreign experts as lacking transparency and should be substantially improved or repealed.

Conclusions The Ministry for Development of Economy, Trade and Agriculture of Ukraine registered with the Verkhovna Rada of Ukraine the draft Law of Ukraine "On Amendments to Certain Legislative Acts of Ukraine" (reg. No. 2565 of 10/12/2019), whereby the Law "On Distribution of Copies of Audiovisual Works, Phonograms, Videograms, Computer Software, Databases" is to be declared no longer in effect, while the relevant amendments and modifications are to be made to the Law of Ukraine "On Copyright and Related Rights", to the Criminal Code of Ukraine, the Criminal Procedure Code of Ukraine, and the Code of Ukraine on Administrative Offences.

\section{References}

1. Avdieieva H.K., 2006. Forensic examination of counterfeit audiovisual products (based on criminal case files). Monograph, ed. Professor V. Yu. Shepitko. Kharkiv: Pravo, available at http://dspace.nlu.edu.ua/bitstream/123456789/648/1/AvdeevaMono.pdf

2. The Law of Ukraine "On Distribution of Copies of Audiovisual Works, Phonograms, Videograms, Computer Software, Databases", available at https://zakon.rada.gov.ua/laws/show/1587-14

3. Lelotko Yu. I. The official web portal of the Verkhovna Rada of Ukraine. Available at http://elar.naiau.kiev.ua/jspui/bitstream/123456789/9057/1/\%D0\%A1\%D0\%A3\%D0\%94\%D 0\%9E\%D0\%92\%D0\%9E\%D0\%95\%D0\%9A\%D0\%A1\%D0\%9F\%D0\%95\%D0\%A0\%D0\%A2\%D0\%9D\%D0\%90_p 218-220.pdf

4. The Order No. 591 of the Ministry of Internal Affairs of Ukraine dated 17/07/2017. The official web portal of the Verkhovna Rada of Ukraine. Available at https://zakon.rada.gov.ua/laws/show/z1024-17 
5. The draft Law of Ukraine "On Amendments to Certain Legislative Acts of Ukraine", registration No. 2565 of 10/12/2019. The official web portal of the Verkhovna Rada of Ukraine. Available at http://w1.c1.rada.gov.ua/pls/zweb2/webproc4_1?pf3511=67591

6. Chvankin V.A. Osobennosti otdel'nykh kriminalisticheskikh issledovanii produktsii, obladaiushchei priznakami kontrafaktnosti : posob. [Features of individual forensic studies of products with signs of counterfeiting: handbook] / V.A. Chvankin, A.L. Poskreblo. - Minsk, 2005.

7. Chvankin V.A. Praktychni aspekty kryminalistychnyh doslidzhen' produkcii' $z$ oznakamy kontrafaktnosti : metod.rek. [ Practical aspects of forensic studies of products with signs of counterfeit: method.] / V.A. Chvankin, A.V. Kofanov. - K. : KYJ, 2008.

8. Chvankin V.A. Metodika tekhniko-kriminalisticheskogo issledovaniia poligraficheskogo oformleniia $i$ elementov zashchity magnitnykh $i$ opticheskikh nositelei informatsii, soderzhashchikh ob"ekty intellektual'noi sobstvennosti, v tseliakh ustanovleniia tekhnicheskikh priznakov kontrafaktnosti [Methodology of technical and forensic investigation of printing design and protection elements of magnetic and optical information carriers containing intellectual property objects, in order to establish technical signs of counterfeiting] / V.A. Chvankin // Kriminalisticheskoe obespechenie rassledovaniia i raskrytiia prestuplenii: nauch. tezisy, vystup. i soobshch, Kiev, 30 apr. 2010 g. / Kiev. nats. un-t vnutr. del Ukrainy. K.: KII, 2010. - S. 150-155.

\section{Translation of references to the original language}

6. Чванкин В.А. Особенности отдельных криминалистических исследований продукции, обладающей признаками контрафактности : пособ. / В.А. Чванкин, А.Л. Поскребло. - Минск, 2005.

7. Чванкін В.А. Практичні аспекти криміналістичних досліджень продукції 3 ознаками контрафактності : метод.рек. / В.А. Чванкін, А.В. Кофанов. - К. : КИЙ, 2008.

8. Чванкин В.А. Методика технико-криминалистического исследования полиграфического оформления и элементов защиты магнитных и оптических носителей информации, содержащих объекты интеллектуальной собственности, в целях установления технических признаков контрафактности / В.А. Чванкин // Криминалистическое обеспечение расследования и раскрытия преступлений: науч. тезисы, выступ. и сообщ, Киев, 30 апр. 2010 г. / Киев. нац. ун-т внутр. дел Украины. К.: КИЙ, 2010. - С. 150-155. 
DOI 10.32370/IA_2020_01_10

\title{
Missed Opportunities for Previous Examinations under the Current Criminal, Civil and Criminal Procedure Codes of Ukraine
}

\begin{abstract}
Atamanchuk Volodymyr
PhD of Juridical Sciences, Chief of Department of Forensic Support and Forensic Expertise of the National Academy of Internal Affairs, Kiev, Ukraine ORCID ID 0000-0002-1464-7871 atamanchykvlad@ukr.net

\section{Pavlovska Nataliia}

PhD of Juridical Sciences, Associate Professor, Professor of Department of Civil Law and Process of the National Academy of Internal Affairs, Kiev, Ukraine ORCID ID 0000-0003-3311-0364 wwwpav@gmail.com
\end{abstract}

\section{Kulyk Maryna}

PhD of Juridical Sciences, Associate Professor of Forensic Support and Forensic Expertise of the National Academy of Internal Affairs, Kiev, Ukraine ORCID ID 0000-0003-1373-6749 coolss777@ukr.net

\section{Symchuk Anatolii}

Senior Teacher of Forensic Support and Forensic Expertise of the National Academy of Internal Affairs, Kiev, Ukraine ORCID ID 0000-0002-8663-8210 symchukas@gmail.com

\section{Filipova Natalia}

Asistant of the Departament of Law of Prydunai Branch of Private Jointstock Company «Higher Educational Institution of Interregional Academy of Personnel Management», Izmail, Ukraine ORCID ID 0000-0001-7132-8343 filinatalja2310@ gmail.com

\footnotetext{
Abstract

The article presents the author's viewpoint on the assessment of positive and negative aspects beyond the procedural status of the expert's opinion, as well as current prospects for the implementation of the previous positive experience.

The key purpose, for which the conduct of examinations and provision of expert opinions was intended, was to implement the principle of rapid (prompt) delivery of evidentiary information for the purpose of detection
} 
and investigation of crimes. Most often, the conduct of examinations and provision of opinions by experts were ordered in the areas of forensic examination, one of which included forensic weapon analysis.

Keywords: investigator, examinations, expert's opinion, forensic weapon analysis.

Introduction Naturally, the sooner the investigator (operative officer) received most of the useful information on the case, the higher the chances were that the crime would be solved and the perpetrator exposed. Therefore, these examinations were largely conducted at the initial phase of the investigation, at a stage of initiation of a criminal case or (before the start of the criminal process) at a stage of operative and investigative activities focused on identifying the crime, i.e., when the essential evidentiary information reflected in the physical traces of the crime had to be obtained as soon as possible, while no conditions, possibilities or legal prerequisites existed for the conduct of a forensic examination.

The nature of any performed forensic weapon analysis, finalised in such a document as an expert opinion, was both procedural and non-procedural. Its existence or absence in the legal framework depended on the stage of the process at which it was ordered and on the agency (person) initiating such analysis [1].

At present, certain challenges arise in determining the items of crimes, when categorised under Articles 262-264, 410 of the Criminal Code of Ukraine.

The provisions of Articles 262-264, 410 of the Criminal Code of Ukraine (the "CCU") criminalise, in particular, illegal actions related to dealing with firearms, ammunition, explosive devices (items of crime) that, for the purpose of categorising certain items as items of crime under these articles are analysed during forensic examinations of weapons and explosives.

Thus, Article 263 of the CCU defines two types of weapons: firearms (other than smooth-bore weapons) and cold weapons, although no definition of the types of ammunition or explosive devices is given.

The Resolution No. 3 of the Plenary Assembly of the Supreme Court of Ukraine "On judicial practice in the cases of theft and other illegal handling of weapons, ammunition, explosives, explosive devices or radioactive materials" dated 26/04/2002 clarifies that:

firearms shall mean all types of combat, sports, and rifled hunting weapons, both commercially manufactured and home-made or converted, which use the pressure force of gases formed by combustion of an explosive substance (gunpowder or other special 
combustible mixtures) to fire a shot;

ammunition shall mean cartridges for rifled firearms of various calibres, artillery shells, bombs, mines, grenades, warheads of missiles and torpedoes and other assemblies, equipped with explosives and intended for firing firearms or making an explosion;

explosive devices shall mean home-made or industrially manufactured disposable products, specially prepared and, under certain circumstances, capable, through the use of chemical, heat, electrical energy or physical action (explosion, impact), of generating effects - i.e., to cause death, bodily injury or significant material damage - through the release, dispersion or exposure to toxic chemicals, biological agents, toxins, radiation, radioactive materials or other similar substances.

In addition to the said items of crime, experts nowadays also examine disposable and reusable rocket launchers, artillery systems (guns and mortars), flamethrowers, portable and mobile (combat vehicles) anti-tank missile and anti-aircraft missile systems, their ammunition and missiles that, at least, are not defined by the above regulations. Electroshock devices with certain characteristics remain beyond the categorisation of weapons [2].

Given the above, forensic categorisation of weapons requires more precise definitions, based on the expert practice and provisions of regulatory documents that govern it, first of all, the Instructions on ordering and conducting forensic examination and expert analysis, and the Methodological guidelines on preparing and ordering forensic examination and expert analysis, approved by the Order No.53/5 of the Ministry of Justice of Ukraine dated 08/10/1998, as well as similar regulatory documents from the expert services of the Ministry of Internal Affairs of Ukraine (the "MIA") and the Security Service of Ukraine (the "SSU").

It is thus proposed that the forensic categorisation of weapons and their ammunition be updated to read as follows: cold weapons; non-kinetic weapons (electroshock devices and nonrocket flamethrowers); small arms (industrially manufactured or home-made) and their ammunition; reusable grenade launchers and flamethrowers, rounds (grenades) for them; disposable grenade launchers and flamethrowers; artillery pieces (guns, howitzers, mortars) and their ammunition; rocket weapons (rocket systems (RS), multiple launch rocket systems (MLRS), portable and mobile anti-tank missile systems (ATMS) and anti-aircraft missile systems (AAMS), their ammunition and missiles.

Based on the weapon categorisation, items of the expert examination of weapons and 
explosives have been defined, i.e., "Items of analysis in the expert examination of weapons."

It is suggested that the current list (all types of combat, sports, rifled hunting weapons), with regard to new items, be revised to read as follows: 1. Small arms (industrially manufactured or home-made) and their ammunition; 2. Reusable grenade launchers and flamethrowers; 3. Cold weapons; 4. Non-kinetic weapons (electroshock devices and nonrocket flamethrowers); 5. Artillery pieces (guns, howitzers, mortars); 6. Rocket and missile weapons (rocket systems (RS), multiple launch rocket systems (MLRS), anti-tank missile systems (ATMS), mobile and portable anti-aircraft missile systems (AAMS) and modifications thereof).

\section{Items of analysis in the expert examination of explosives}

The current list (artillery shells, bombs, mines, grenades, warheads of missiles and torpedoes and other assemblies, equipped with explosives and intended for firing firearms or making an explosion) be amended to include the following: grenades (rounds) for reusable grenade launchers and flamethrowers; disposable grenade launchers and flamethrowers, torpedoes, missiles.

Items of analysis in the expert examination of weapons and explosives may also include fragments of the above-listed items of crime, which have remained after their use.

Therefore, to ensure adequate regulatory support for circulation of weapons in this country, laws must be drafted and adopted to govern the circulation of military and specialpurpose weapons, as well as the circulation of civilian weapons.

Furthermore, the list of expert specialities in the field of expert examination of weapons has been updated: "Expert examination of weapons."

1. Analysis of small arms and their ammunition, reusable grenade launchers and flamethrowers (Speciality 3.1).

2. Analysis of traces of small arms and their ammunition, reusable grenade launchers and flamethrowers, traces and situational circumstances of their application (Speciality 3.2).

3. Analysis of cold weapons (Speciality 3.3).

4. Analysis of non-kinetic weapons, traces and situational circumstances of their application (Speciality 3.4).

5. Analysis of artillery pieces, traces and situational circumstances of their application (Speciality 3.5). 
6. Analysis of rocket and missile weapons, traces and situational circumstances of their application (Speciality 3.6).

Further efforts to improve statutory instruments that govern expert examination of weapons and explosives would require a revision of the methodologies for analysis of those objects that may constitute items of crimes under Articles 262-264, 410 of the Criminal Code of Ukraine, along with subsequent refinement of these methodologies.

Therefore, during the conduct of (at the stage of conducting) operative and investigative activities (the stage where elements of crime are identified), an analysis would be ordered at the request of operative units $(\S \S 4.1-4.6$ of the Guidance on the operation of the forensic expert service of the Ministry of Internal Affairs of Ukraine); this analysis was not regarded as a procedural one, since it was taking place beyond the framework of a criminal process (conduct of investigation).

Any analysis was regarded to have been regulated procedurally, if ordered after the criminal case had been initiated - at a stage of preliminary or judicial investigation, on the basis of attorneys' applications (Article 6 of the Law of Ukraine "On the Bar", Article 48 of the "previous" Criminal Procedure Code of Ukraine (the "CPCU")), at the request of customs authorities, notaries $(\S 3$ of the Instructions on ordering and conducting forensic examinations, as approved by the Order of the Ministry of Justice of Ukraine No. 53/5 dated 08/10/99), following the applications filed by organisations and members of the public (the Law of Ukraine "On Forensic Examination”).

From a legal viewpoint, the most contentious issues of non-procedural analysis have been due to the following: performing the analysis of items and substances that could acquire the status of evidence prior to expert examination as a procedural action; performing the analysis outside the framework of criminal process, i.e., the impossibility for parties to the investigation to control the material evidence; a unique nature of the analysis of certain items, in particular, in the field of "forensic weaponology" involving home-made weapons and homemade cartridges that could only be recognised as firearms or ammunition following a forensic experiment (experimental firing), which involved the expenditure (destruction) of a certain number of these items (the analysis would involve only those techniques that neither alter the appearance or properties of the analysed items nor result in their loss or preclude subsequent expert examination under $\S 5.3 .4$ of the Guidance. The experts conducting such an analysis 
followed the relevant advice that, where necessary, not all the analysed items should be used up, as another expert institution might be later charged with performing the analysis); "blanket" rejection of the expert's opinion as a source of evidence in criminal proceedings; the person conducting the analysis is granted the status of an expert whose written opinion lacks a written warning against giving a knowingly false opinion. A good example of this is the following situation: on the instructions from an investigator of the oblast prosecutor's office, a forensic analysis of a sawn-off shotgun was performed by an expert institution of the Ministry of Internal Affairs of Ukraine, finalised as an expert opinion. The sawn-off shotgun was found to be a firearm suitable for firing. It subsequently became necessary to file charges under Article 263 of the CCU in addition to those that have already been filed. Due to the fact that the expert opinion could not be used as the basis for the indictment, the judge, upon submission from the prosecutor, issued a ruling to perform a forensic ballistic examination, albeit at another expert institution in the MIA system.

In this particular instance, the progress and outcomes of the examination should have been recorded, meaning that where the examination is not possible without the use of the techniques that change the appearance of the examined items, their initial state is to be additionally recorded and photographed, and a summary of the examination process is to be provided, containing references to the techniques and technical means applied, along with the description of the identified features and their assessment results. The nature of the changes and the amount of the materials used should also have been indicated. All this should have been attested by a signature of the person performing the examination. Taken together, these requirements would have allowed for the essential principle in the law of evidence, i.e., reliability (validity as an integrity of evidentiary properties of the investigated traces, and the reliability of the actual data obtained during their examination) to be maintained [3].

At the same time, any examination of physical sources of evidentiary information, which may subsequently acquire the status of physical evidence, should under no circumstances, in this author's opinion, be moved beyond the legal framework or conducted "covertly" outside legal formalities, without recording the progress and outcomes of such examination in legal (procedural) documents. Handling the sources of evidentiary information should at all times be open and recorded with the use of procedural means. The record must be complete, comprehensive and continuous, beginning with the identification of a physical item 
that has a probative value and ending with its examination. Otherwise, no guarantees could exist that the evidentiary properties of a seized item have remained intact and the item itself may represent a reliable source of information and means of finding the objective truth.

Thus, a contradictory nature of the expert opinion has been observed, with regard to the possibility of its expanded presentation and compliance with the principles of reliability and admissibility of physical evidence against the background of the preliminary examination performed outside the criminal process; accordingly, the expert opinion required reviewing, rather than revoking its status in the context of the criminal process in Ukraine.

Therefore, since those items that have the status of physical evidence or may acquire this status later are subjected to examination, any preliminary analysis of such items should not be performed covertly. This examination must be performed within the framework of legal formalities, thereby guaranteeing the reliability of the analysis, the validity of its conclusions, and the integrity of the investigated evidence. In this context, the experts' opinions should have been given the status of an evidentiary source. This could have been particularly pertinent to the properly registered items that had been sufficiently established procedurally and might have acquired the status of physical evidence, whose examination is unique and, at the same time, should be done fast. In this case, it is impractical, from a legal viewpoint and for reasons of saving the expert's and the investigator's time, efforts and resources, to perform, under a respective ruling, any complete "automatic" re-examination following a preliminary analysis that should be granted the appropriate legal status ("sources of physical evidence").

In view of the European standards of justice being introduced in this country, an urgent need exists for improvements to the administrative and legal principles of conducting a forensic examination, processing its results and for bringing them closer to European standards. This approach should ensure prompt handling of analysed items, while observing the principles of legality, reliability and objectivity. The prerequisite for transitioning to a simplified option for finalising the expert opinion is rooted in the common format of examining the sources of evidentiary information, which is determined by the commonality of the content and methods of examination, since the analysis methodology at the stage of preliminary examination is no different from the one used at the stage of expert examination. The simplified nature of a finalised expert opinion is manifested in the absence of an analysis section (description of the analysis process) in such an opinion. An expert opinion may be used in this form where a need 
exists for a fast and immediate examination, while the examination itself does not entail annihilation of the examined items with destructive methods. The expert must provide a descriptive section of the analysis at the request of the initiator of the forensic examination or the court (where not initiated by the court) [4].

This simplified option for drafting the expert opinion is also supported through implementation of a management system according to the requirements of the DSTU ISO/IEC 17025 standard.

Compliance with the DSTU ISO/IEC 17025 requirements enables the implementation of such key principles of the MIA expert service operation as continuous improvements in the quality of examinations, along with strict monitoring of the reliability of their outcomes.

The improved reliability of the studies is achieved by precise functioning of the personnel training and retraining system, application of advanced research techniques (methods), introduction of modern measuring devices and test equipment and their proper maintenance, observance of strict confidentiality, professional secrecy and the customers' rights of ownership.

Similar to any introduction, intended to improve the efficiency of the unit's operation and the quality of its examinations, this introduction also has its purpose, goals and objectives.

The introduction of a management system has the purpose of guaranteeing high quality of measurements, tests and analyses, thereby ensuring objective and reliable results; unconditional observance of the requirements imposed by regulations (standards), and of investigative techniques, as well as of the rules for the use of measuring devices and test equipment; creating the required environment for examinations; the appropriate level of the personnel competence; prompt fulfilment of the customers' requests for examinations at minimum costs; carrying out continuous self-analysis (audit of in-house activities), as well as being personal responsible for the quality of the work performed.

The key goals in the policy of implementing the management system include: maximum satisfaction, within one's competence, of customers' needs with the quality of laboratory services and precise discharge of all obligations before the customers; stable quality; ensuring the customer's appropriate confidence in quality; confidentiality conditions when performing examinations; objectivity of the outcomes, validity of conclusions, opinions and 
interpretations of the results; carrying out continuous self-analysis (audit of in-house activities) to achieve ongoing improvements in the in-house management system.

Introduction Functioning of the management system is supported by the following factors: availability of premises to perform examinations, of the necessary test equipment, measuring devices and other technical facilities to conduct analyses in full conformance to the statutory, methodological and technical documents; the statutory, methodological and technical documents that govern requirements on the examined items, methods of their examination, as well as requirements on the examination tools and the procedure for their application; skilled personnel, competent in the matters of staging and performing examinations, processing and reporting of outcomes, as well as comfortable psychological climate in the team.

\section{References}

1. Kofanov A. V., 2015. Specifics of expert's errors and ways to prevent them in a forensic ballistic examination. In: Forensic Science and Examinations - An inter-agency instructional research compendium. Ministry of Justice of Ukraine, KNDISE. Kyiv. Vol. 60. pp. 275-287.

2. Kofanov A. V., 2013. Certain aspects of the challenges in the training of forensic experts, their interaction with senior officials and assessment of their analyses. In: Forensic Bulletin - Academic and practical compendium. Chief editor V. Cherniei. DNDEKC, MIA of Ukraine; NAIAU. Kyiv: A to Z Printing House. No. 2 (20). pp. 107-116.

3. Kofanov A. V., 2015. Deficiencies in psychological and legal (professional) training of a forensic expert as a prerequisite for occurrences of experts' errors. In: Forensic Bulletin Academic and practical compendium. Chief editor V. Cherniei. DNDEKC, MIA of Ukraine; NAIAU. Kyiv: A to Z Printing House. No. 1 (23). pp. 72-79.

4. Kofanov A. V., 2018. Debatable nature of an expert opinion or its equivalent (past and future). In: Current issues of standardisation and accreditation of trace evidence and weapon examination - Proceedings of the inter-agency academic and practical seminar (Kyiv, 12 January 2018). KNDISE. Kyiv. pp. 30-34. 
UDC 340.13

\title{
Immediate Steps of Judicial Reform in Ukraine
}

\author{
Aleksandr B. Kapustin, lawyer, \\ Founder of "The European Union of Christian Attorneys", Odessa, Ukraine
}

\begin{abstract}
The United State Agency for International Development (USAID), the Center of the European researches, the International Monetary Fund (IMF), OSCE, the Council of Europe, GRECO carried out the Ukrainian reforms project and other projects together with the Ukrainian authorities, allocated the huge amounts of money to Ukraine for carrying out reforms in the field of the right. Dull haste of the previous power led to the fact that for universal dismissal of judges and enrollment for work of new personnel in courts: many courts operating in Ukraine, including the Supreme Court of Ukraine were liquidated. As now, it is visible from the decision of the Constitutional Court of Ukraine that the reforms were undertaken contrary to provisions of the Constitution of Ukraine. Therefore, with the Decision of the Constitutional Court of Ukraine N 2-p/2020 dated 18.02.2020 we state new and rather next collision of reforming of the right in Ukraine. All this is absolutely and certainly reflected in the constitutional guarantees of independence of judges, affects both prestige and trust of citizens to judicial authority and morale of judges. Monetary grants of the international donors are once again spent by the authorities of Ukraine not reasonably and not effectively. In inferior courts, some citizens dare to pour paint in buildings of the courts over judges, to spit the Themis's representatives. Periodically the power of Ukraine tries to review (reduce) the monetary reward to judges. As we have noticed it above, all this affects both prestige of employees of the court, and trust of citizens to Themis. The personnel hunger in courts of Ukraine is catastrophic; courts due to the lack of judges are systematically closed. The reformed procedural legislation provides the introduction of the institute of jurymen; however, citizens do not want to be directly involved in sentences of justice and everywhere personnel hunger in the matter is seen. The immediate measures proposed in this work will lead to quick and effective overcoming the crisis in judicial reform.
\end{abstract}

Keywords: Judicial reform, the judicial system, justice, independence of judges, guarantees of legal proceedings.

\section{Introduction}

The legislation and practice of its application inevitably absorb in themselves the previous experience of the human civilization. In the state and legal sphere - it is, first of all, those its stages when democratic principles of the social system and such progressive political and legal ideas as supremacy of law, the constitutional state, separation of the authorities, the priority of the rights and freedoms of the person over other social values began to get into public consciousness and practice gradually. At the same time, it is pertinent to remember the known sayings: "New is well forgotten old" and "History teaches us to the fact that it teaches us to nothing". The last in its pointed polemic form emphasizes inability or unwillingness of some statesmen and scientists-lawyers to wholly make use of historical experience of state and legal construction, including domestic. There can be the question: about what loan of experience in the state and legal sphere can there be a speech concerning the absolutist Russian Empire, in which the most of present Ukraine or the totalitarian Soviet state had been. But the ambiguously negative answer to this question 
would be wrong and in essence anti-historical. Therefore, first, it is always possible to find something positive in complex systems of legal regulation and to use it in modern conditions. Second, it is possible to avoid mistakes which had been made in due time by our predecessors. [1, pp. 309-310].

The judicial reform is the set of interconnected, system, normative fixed at the nationwide level (by codification included) changes in the area of the judicial system and the legal proceedings undertaken for the achievement of maximum efficiency of functioning of the judicial system and performed during the historically limited period.

The declaration on the state sovereignty of Ukraine adopted by the Supreme Council of the Ukrainian SSR on July 16, 1990, proclaimed the state sovereignty of Ukraine. The declaration proclaimed: "All citizens are equal in the eyes of the law, irrespective of the origin, the social and property status, the racial and national identity, sex, education, political views, religious beliefs, the sort and character of occupations". On August 24, 1991, the Supreme Council of the Ukrainian SSR adopted the Act of Declaration of Independence of Ukraine and adopted the Resolution "About Declaration of Independence of Ukraine" which announced Ukraine as the independent democratic state. From the moment of formation of modern Ukraine, that is for nearly three decades, in Ukraine reforms are undertaken. A legislator of Ukraine continuously reforms judicial authority.

At the heart of judicial and legal reform in Ukraine accepted by the Supreme Council of Ukraine on April 28, 1992, it was indicated that need of judicial and legal reform is caused by the fact that courts endure the deep crisis caused by many factors which negatively influence their activity. The judicial reform undertaken in Ukraine was intended to make the number of cardinal changes, to accept precepts of the law which in the set shall change the judicial system of Ukraine, that is the system of the judicial organization and the order of permission of legal cases. The crucial elements of the reform are reorganization of courts and reduction of the staff of judges; updating and clarification of the judicial body - the qualified estimation of the effective judges; and also competitive selection: both candidates for the judgeship, and effective judges at their personnel movement (change of court or specialization of the judge); independence of judicial management: the public authority to which competence including also forming of the judicial body and execution of authority punishments - the Supreme Council of Justice are referred is created; optimization of legal proceedings: electronic legal proceedings, remote legal proceedings, increase in court fee, lawyer monopoly for representation in courts, introduction of the concept of "standard or model affairs", modification of the existing procedural codes of Ukraine. [2].

So, as we have established above, for all history of the existence of modern Ukraine, a legislator of Ukraine undertakes in the country infinite reform in the field of justice. At the same time, reforms in the field of the judicial system, legal professions that is - not specialists in that area are engaged in the legal reforms. Many projects of schools of sciences 
of jurisprudence of Ukraine and remained unconsidered by the Supreme Council of Ukraine as in the most cases all next "large-scale" reforms are undertaken spontaneously - to please to the next won political force and have rather populist character, without setting as the purpose improvement of quality of life of the Ukrainian people, and in this case, their right to fair trial and protection of the civil and constitutional rights.

The Constitutional Court of Ukraine notes that renaming of the authority fixed in the Constitution of Ukraine - the Supreme Court of Ukraine - may not happen without transfer of judges of the Supreme Court of Ukraine into positions of judges of the Supreme Court as there are no distinctions between the legal position of a judge of the Supreme Court of Ukraine and a judge of the Supreme Court, and withdrawal of the word "Ukraine" - the own state - from the verbal structure "The Supreme Court of Ukraine" may not be the basis for release of all judges of the Supreme Court of Ukraine or their transfer to another court, especially a lower court. The Constitutional Court concluded that the judges of the Supreme Court of Ukraine should continue to perform the powers as the judges of the Supreme Court. Therefore, the actual differentiation of the judges of the Supreme Court of Ukraine and the Supreme Court does not accord with the principle of the irremovability of judges; it is the compound constitutional guarantee of the independence of judges - the Decision of the Constitutional Court of Ukraine N 2-p/2020 dated 18.02.2020. [3].

On February 18, the Constitutional Court adjudged liquidation of the Supreme Court of Ukraine during the judicial reform of 2016 as unconstitutional. By its decision of 18.02.2020, the Constitutional Court adjudged provisions of such clauses of Section XII "Final and Transitional Provisions" of the Law "On the Judicial System and the Status of Judges": - 7 - about liquidation of the Supreme Court of Ukraine; - 14 - concerning competition to the position of the judges of the Supreme Court; - 25 - about the right to monthly perpetual monetary pay - to not correspond the Constitution. Thus, the Constitutional Court of Ukraine concluded that the judges of the Supreme Court of Ukraine should continue to perform the powers as the judges of the Supreme Court. The relevant provisions of the Law "On the Judicial System and the Status of Judges" become not applicable from the date of adoption of this decision by the Constitutional Court of Ukraine. However, the Constitutional Court in the decision did not call into question competition on screening of the judges in the new Supreme Court. Besides that, the Constitutional Court of Ukraine did not adjudge creation of the new Supreme Court (that is the clauses of the Law based on which, it was created) as unconstitutional.

As it is stated above, reforms in the field of the right in Ukraine did not yield a positive result, which citizens of Ukraine had expected. We offer immediate emergency steps, which will lead to recovery of prestige of the occupation of judge, including to the material guarantee of the salary of judges as the part of the legislative package of independence of judges, and in turn will result in the efficiency of the judicial system, we suggest to make the following changes to the legislation of Ukraine: 
1. To make the following changes to paragraph 3 of article 135 of the Law of Ukraine "On the Judicial System and the Status of Judges": The basic amount of the official pay rate of a judge is:

1) judges of local court - 50 (instead of specified now 30) living wages for ablebodied persons, the amount of which was established for January 1 of a calendar year;

2) justices of appeal, judges of the supreme specialized court - 60 (instead of specified now 50) living wages for able-bodied persons, the amount of which was established for January 1 of a calendar year;

3) judges of the Supreme Court - 70 (instead of specified now 55) living wages for able-bodied persons, the amount of which was established for January 1 of a calendar year.

To make the following changes to paragraph 7 of article 135 of the Law of Ukraine "On the Judicial System and the Status of Judges": Monthly surcharge for scientific degree of Candidate (Doctor of Philosophy) or Doctor of Sciences with the corresponding specialty at a rate of respectively 20 and 25 (instead of specified now 15 and 20) per cent of the official pay rate of the judge of the relevant court is paid to the judges.

Monthly surcharge for the state awards of Ukraine - the honorary title: The honoured lawyer of Ukraine, and (or) the Honored academic and technical figure of Ukraine (now it is not paid) of 10 per cent of the official pay rate of the judge of the relevant court is paid to the judges for each award.

2. To state article 376 of the Criminal Code of Ukraine in the following version:

1). Intervention in any form in the activity of the judge with the purpose to interfere with the accomplishment of service duties by him/her or to achieve pronouncement of an illegal decision is punished by corrective works for a period of from one year up to two years, or arrest for a period of up to six months.

2 ). The same actions, if they interfered prevention of a crime or detention of a person who had made it, or executed by a person with use of the official position, are punished by deprivation of the right to hold certain positions and to be engaged in the certain activity for a period of up to five years and imprisonment for a period of from three to five years.

3. To state article 377 of the Criminal Code of Ukraine in the following version:

1) Threat of murder, violence, destruction or damage of property concerning a judge, a juryman or a juror, and concerning their close relatives in connection with their activity connected with implementation of justice is punished by restriction of freedom for a period of up to three years or imprisonment for a period of from three to five years. 
2) Intentional causing a judge, a juryman or a juror or to their close relatives beating, bodily damages of light or medium severity in connection with their activity connected with implementation of justice is punished by restriction of freedom for a period of up to five years or imprisonment for a period of from five to eight years.

3). Intentional causing heavy bodily damage to a judge, a juryman or a juror or their close relatives in connection with their activity connected with implementation of justice is punished by imprisonment for a period of from eight to fourteen years.

4. To state article 378 of the Criminal Code of Ukraine in the following version:

1). Intentional destruction or damage of the property belonging to a judge, a juryman or a juror or their close relatives in connection with their activity connected with implementation of justice is punished by imprisonment for a period of from five to eight years.

2). The same actions made by arson, explosion or another all-dangerous method, or which entailed death of people or other heavy effects are punished by imprisonment for a period of from eight to fifteen years.

\section{Conclusions:}

Reforms in the field of justice, which are undertaken systematically in Ukraine, have spontaneous character; numerous scientific lawyers are not involved in their development, as the central element the political idea of authorities in power is often brought in the basis of those reforms. The steps specified by us will without delay render essential measures: on recovery of independence of legal proceedings in Ukraine, to efficiency of any further reforms which would be obvious to be carried out the newly elected power of Ukraine, to recovery of trust of citizens to judicial authority of Ukraine, recovery of prestige of the occupation of a judge and respectively to filling a judicial authority by the professional personnel. Moreover, the problem of hunger for the personnel, and the actual absence of wish of citizens of Ukraine to become jurymen would also respectively be solved positively.

\section{References}

1. Yu. E. Polianskii. Use of historical and analytical approach in the course of judicial reform in Ukraine. / Yu. E. Polianskii // Urgent problems of the state and right. - Odessa: "Legal literature", No. 45, 2009. - pp. 309-310.

2. A. B. Kapustin. Judicial reform in Ukraine: Legal collapse and its reasons. / A. B. Kapustin // Constitutional state. - Odessa: "Phoenix", No. 29, 2018.

3. The decision of the Constitutional Court of Ukraine of 18.02.2020 on the case on the constitutional representation of the Supreme Court of Ukraine N 2-p/2020. 
Case N 1-15/2018 (4086/16) [The electronic resource]. - Access mode: http://www.ccu.gov.ua/sites/default/files/docs/2_p_2020.pdf?fbclid=IwAR3a4SjQ htgFfUd_vhUzdeU4c_4Kk-Cv-azXKNgWdxw9ufrDY0vZP1hRE9k2 


\title{
Unplugging in Language Learning and Teaching \\ Laptinova Yuliia \\ Kharkiv I. P. Kotlyarevsky National University of Arts \\ ORCID ID \\ https://orcid.org/0000-0002-4175-0858
}

\begin{abstract}
This article explores the possible way to make English Language Learning and Teaching more efficient among university students. Viewing language as a skill to acquire not a subject to study, the paper suggests Teaching Unplugged as an approach that empowers learners with the skills they need to speak in the target language outside the classroom. The article analyses psychological learning theory, which explains how people might acquire knowledge and learn. It also reviews some main principles and benefits of Teaching Unplugged as well as some of the issues that serve as a disruption for teachers and students. The article outlines one technique that could be used to unplug the classroom.
\end{abstract}

Key words: Teaching Unplugged, language learners, speaking skills, constructivism, 'Dogme’ moment.

\section{Introduction}

Nowadays the majority of English learners are very motivated and goal-oriented. They learn another language in order to be fully functional in our global society. Goal-oriented students are demanding, and this motivates teachers to do their best to meet the goals of all their students.

Reflecting on my teaching at the University, I have noticed that the over-dependency on external obstacles (like pre-set syllabus, fixed course book, pre-decided order and pace, pre-selected grammar items) as well as the overuse of language learning resources available to you at a click of a button serve as a distraction for the learning opportunities in the classroom. Language Teaching and Learning should be treated as a process of jointly constructing speaking skills out of the talk yet materials overload and restrictions prescribed by a course hinder real classroom communication. Therefore, it is timely to enrich classroom activities and procedures by relinquishing the course book in exchange for the content provided by spontaneous conversation that occurs naturally amongst teachers and learners. What if we untether the lesson i.e. make our lessons free from course books, externally set syllabus and restrictive materials. We advocate the adoption of Unplugged method ("Dogme") which welcomes teachers' willingness and authority to keep this complicated balancing act between curriculum regulation and curriculum freedom. Luke Meddings and Scott Thornbury coined the term "Unplugged" ("Dogme") having adapted the philosophy of the film movement to English Language Teaching. The idea was to encourage the Language Learning and Teaching without all the photocopies, visual aids, and other handouts what is seen as excesses in the classroom and to take 
teaching back to its essence: the communication between teachers and students "making the most of minimal means" [3]. In their article "Teaching Unplugged: Dogme in English Language Teaching" Luke Meddings and Scott Thornbury state the precepts that lay the foundations of the Dogme Approach and, thus, establish grounds to classroom activities and procedures [5]. An education writer Janelle Cox in her article "Classroom Management: Benefits of Being Unplugged" states a few unexpected classroom management benefits language learners will have from an unplugged classroom, as well as ideas for how to make it work [1]. Ken Lackman has developed a method for Teaching Unplugged, which he has named Conversation Activated Teaching (CAT), implementing the main features of Dogme [3]. Lisa Dold views Teaching Unplugged not as a methodology to follow but rather as a mindset, which can be adopted by teachers who take issue with the proliferation of published materials or feel overwhelmed with a plethora of coursebook supplements [2]. Sharmila Pixy Ferris and Hilary Wilder, Professors at William Paterson University provide techniques to help teaching and learning in an age where technology untethers instruction from the classroom, from semester seat-time, and from a single source of expertise [7].

This paper intends to highlight and explain the main principles and ideas that underpin Teaching Unplugged; discuss constructivism learning theory as a ground for Teaching Unplugged; define the teaching/learning context required for a successful implementation of Teaching Unplugged; expose one effective and motivational technique to unplug the class; take a look at benefits of being unplugged.

\section{The main principles of the Unplugged approach}

\section{- $\quad$ Conversation as a mode of language learning.}

The most prominent feature of Teaching Unplugged is that it puts conversation at the heart of language learning [6]. This statement infers that conversation is both the process and the product of language learning. This comes from the belief that conversation is not a product of learning, but necessary for learning to happen. Spontaneous conversations in class allows learners to come up with coherent ideas, and not isolated sentences that might not be relevant. A focus on conversation is believed to better prepare learners to use language in real life. They are more likely to produce utterances in the full context of a conversation than produce a series of pre-fabricated phrases or structures in isolation.

- $\quad$ Generating the emergent language. 
Dogme considers language learning to be a process where language emerges rather than one where it is acquired. The emergent language is the language that the students produce as they are talking spontaneously in class. As language is stimulated rather than simulated, there is no need to follow a syllabus that is externally set. Hence, there is no right order in which grammar structures should be learnt. Any grammar that is the focus of instruction should emerge from the lesson content, not dictate it. It should be more memorable as it comes from students' desire to communicate. It's also possible to let them decide on a language point they would like to work on. Knowledge is, therefore, constructed, instead of being imposed or transmitted by the teacher.

- $\quad$ Diagnosing the language and dealing with it.

The teacher's task is to watch out for learning opportunities that may simply spring up during lessons. If you hear an interesting expression used by a student, call other students' attention to it. Lisa Dold views the teacher's role is to help learners engage with emergent language - that means the teacher should diagnose the language and lexical gaps to target in follow-up work, without having established grammatical or lexical items prior to the lesson [2]. The language that emerges from the teacher-student interaction becomes core language and it is dealt with in exactly the same way teachers have always dealt with language.

- $\quad$ Scaffolding the learning process.

Scaffolding is a key feature of effective teaching, where the teacher continually adjusts the level of his or her help in response to the learner's level of performance [4]. A teacher or more experienced peer is able to provide the learner with scaffolding hints, prompts, drilling exercises and direct instruction to support the development of speaking skills.

- $\quad$ Untethering from pre-set restrictions.

Perhaps most of university teachers today are being plugged into one or another curriculum, predecided order and pace, pre-planned syllabus, pre-selected course book. Moreover, having direct access to lots of resources and social media platforms internationally, they have subjected themselves to published materials, photocopies, supplements, handouts and other embellishments. Every teacher should look critically at the activities in their course books and feel free to reject them, if necessary, in favour of more meaningful and quality time in class. Being unplugged from pre-set distractions fosters interaction in class and allows language to emerge out of the communicative needs, interests and desires of the learners in the room. Scott Thornbury has noticed "By reducing the amount of 
material that is imported into the classroom, the teacher frees the learning space for the kind of interactive, talkmediated learning opportunities that are so crucial for language development" [8].

\section{The psychological ground for teaching unplugged}

Constructivism learning theory as a paradigm for teaching and learning establishes grounds for Teaching Unplugged since it explains how mere conversation leads to students actually learning. Social constructivism was developed by Lev Vygotsky who suggested that: 1) learning is the process of constructing new skills and knowledge only through active engagement with the real-world problem solving. This constructivist view of learning considers the learner as an active agent in the process of knowledge acquisition; 2) learning is a social process of interaction with a more knowledgeable participant. Vygotsky argued that the most effective learning occurs when the 'experts' (teachers) help 'novices' or 'apprentices' to develop their proficiency and stretch their skills [9]. Teachers cannot simply transmit knowledge to students, but students need to actively construct knowledge in their own minds by observing teacher or more experienced peer who are slightly more advanced than they are [4]. Thus, social constructivism suggests that learning is largely a collaborative process, where the new skills are jointly constructed in activity with more capable peers.

\section{Implementation of the Unplugged Teaching}

Success and effectiveness in an Unplugged classroom hinge on:

1) the changes in attitude to language learning and teaching. Students should cultivate the sense of agency rather than seeing learning as the teacher's responsibility. Agency involves the initiative or self-regulation of the learner, the awareness of the responsibility of one's own actions on others. Unplugged Teaching emphasizes that learning only takes place if learners are active and decisive decision-makers involved in the process of knowledge acquisition;

2) the complicated balancing act between curriculum regulation and curriculum freedom. On the one hand, every university has a prescribed curriculum framework that provides all who teach the courses with instructional resources, such as lesson plans. On the other hand, education policies give teachers flexibility to teach in the ways they think best. That is typically left up to teachers to bring life and passion to sometimes unrealistic or boring curriculum.

Passive learners and teachers rigidly enforcing the plan they envisioned for the lesson are completely irrelevant with these types of learning. 


\section{How to unplug the classroom.}

A teacher who wishes to unplug the classroom should create a few unplanned moments in the class so that real communicative needs and language emerge. A "Dogme" moment is a concept describing those moments in a class when you get students speak by selecting suitable stimuli for conversation. A stimulus could be any questions about their professional issues, any controversial topic you think will create discussion, a photo, a set of questions, or even building upon the small talk that is already in progress at the start of the lesson. Students are most engaged by content they have created themselves. Buzz group discussions, debates and vocabulary games all work well with these lessons. Dogme moments can be experienced during many types of activities, which can be done without resources and which engage the students in maintaining a "good vibe" i.e. harmonious informal chatting, which doesn't seem to be something the teacher has planned. Thus, to experience this state, one has to stay away from the attention-robbers common in a modern fast-paced life. A first step would be to turn off your smartphone when speaking.

\section{Benefits of being unplugged.}

There are two important reasons why unplugged teaching will benefit your students.

\section{1) Unplugging makes students more social.}

Consulting electronic devices as well as preaching a point-by-point lesson plan are distracting. We have already become too addicted to the dings of our phones. Holding students' attention, computers disconnect us from alive communication. When you unplug from technology in the classroom you are essentially forcing face-to-face interaction among the students. The more social interaction language learners have without using technology, the better for jointly constructing speaking skills.

2) Unplugging makes students use their minds and imagination. It is easy to just "Google" or search "Pinterest" for an idea, but if you don't have access to technology, then it forces you to use your critical thinking skills.

\section{Conclusions}

The article argues that Language Teaching and Learning should be seen as a way to further improve and refine students' speaking skills by thought-provoking conversation, rather than a way to gain language knowledge prescribed by a course-book. Thus, language teaching and learning should 
be unplugged (disconnected) from distracting, unrealistic or boring external obstacles (prescribed course book, pre-decided order and pace, pre-planned syllabus, any sort of pre-selected materials) which divert attention from real classroom communication. The article advocates the adoption of Unplugged Language Learning and Teaching approach, which seeks to overcome the limitations imposed on teachers in the workplace and welcomes the learner as an active agent involved in the process of co-constructing speaking skills through the 'dialogic' nature of learning. Unplugged Teaching is grounded in social constructivism (a Psychological Theory of Learning) based on the work of Lev Vygotsky who suggested that learners jointly construct skills through interaction with other people under the guidance of an educator or more competent peer.

Successful Unplugged Learning and Teaching takes place if there is an "unplugged" moment - a stimulus of some sort (a photo, a set of questions, a professional issue), which generates a "good chatting vibe". By establishing and maintaining face-to-face cooperative interaction unplugging forces students to use their imagination and critical thinking skills.

\section{References}

1. Cox J. Classroom Management: Benefits of Being Unplugged. TeachHUB.com. Available at: https://www.teachhub.com/classroom-management-benefits-unplugged-classroom.

2. Dold L. Dogme: A Teacher's View. IH Journal of Education and Development. Available at: http://ihjournal.com/dogme-a-teacher's-view-by-lisa-dold.

3. Lackman K. Cat: A framework for dogme. Methods and activities for more effective teaching with less preparation, Toronto. Available at: http://kenlackman.com/files/cathandoutfinal.pdf

4. McLeod S. A. What Is the zone of proximal development? Simply Psychology. Available at: https://www.simplypsychology.org/Zone-of-Proximal-Development.html

5. Meddings L., Thornbury S. Teaching Unplugged: Dogme in English Language Teaching. Delta Publishing, 2009.

6. Parry J. Teaching unplugged: the revitalization of ELT? Carleton University, Ottawa, Ontario. Available at: https://www.semanticscholar.org/paper/TEACHING-UNPLUGGED\%3A-THEREVITALIZATION-OF-ELT-Parry/71e2ebf06a8b251cf25594450d80f9e9ca875157

7. Sharmila Pixy Ferris and Hilary Wilder. Unplugging the Classroom: Teaching with Technologies to Promote Students' Lifelong Learning. Cambridge, Massachusetts: Chandos Publishing.

8. Thornbury S. Speaking Unplugged: 30 Activities for One-to-One Classes. Available at: https://www.onlinetefltraining.com/wp-content/uploads/2013/11/SpeakingUnplugged30Activities.pdf

9. Vygotsky L. S. Mind in Society: Development of Higher Psychological Processes. Revised ed. Edition. Harvard University Press. 


\title{
Teaching Fractions in a Course Basic College Mathematics
}

\author{
Anastasiya Yakunina, Ph.D. \\ Associate Professor of Mathematics \\ Voorhees College, \\ 213 Wiggins Drive, Denmark, SC 29042 USA \\ ayakunina@voorhees.edu
}

\begin{abstract}
The article is dedicated to the problem of optimization of teaching Basic College Mathematics in a college. An author's original method of teaching fractions is presented. Concrete instructions for increasing effectiveness of teaching fractions are given. Special attention is paid to a student-centered approach that is considered in this article as an effective instrument for an improvement of the results of teaching Basic Mathematics at college level. All conclusions and recommendations are based on the results of modern research in Education and on the author's teaching experience (12 years).
\end{abstract}

\section{Key Words}

Basic college mathematics, teaching fractions, methods for increasing effectiveness of teaching math in college, student-centered education

Basic College Mathematics courses are designed for students who don't demonstrate satisfactory mathematical skills on entry-level college placement tests.

The goal of these courses is to help students acquire the necessary math skills for taking more advanced math courses required for their major.

But the instructor may face several serious barriers from the outset. First, students may have studied this material (whole numbers, fractions, etc.) in middle school and high school but still don't understand the concepts. Students often resent having to take these courses again in college. Perhaps the most significant barrier to learning is student dislike of math in general. At the outset of each semester, I ask students whether they like mathematics or not. The results have been the same each year-about $90 \%$ of students say they don't like math because it's difficult and boring.

After reviewing a diverse variety of sources on the problem, and based on my personal classroom experiences, I have created an original "decomposition" method for teaching fractions that seems to alleviate some of the learning barriers mentioned previously.

At the start of his/her unit on fractions, the instructor should give students a clear definition of prime numbers (a natural number greater than 1 whose factors are 1 and the 
number itself) and a definition of a composite number (a number which can be "decomposed" as a product of prime numbers). It is essential to discuss several concrete math problems at this point.

Problem 1. Determine whether the following numbers are prime numbers or composite numbers. If the number is composite, find its prime factors.
a. 5
b. 12

Discussion Strategy:

For (a), ask your students "Can the number 5 be decomposed (represented as a product of other numbers)? Students will answer "No, this number can't be decomposed." So the students have identified 5 as a prime number. It can't be divided.

For (b), ask your students "Can the number 12 be decomposed (represented as the product of other numbers?). Students will answer "Yes it can," recognizing that 12 can be divided into 2 times 6 . You should write $12=2 \times 6$ on the board. Say to the students, "So we've decomposed 12 but is $2 \times 6$ a final answer? Or can we continue the process of decomposition? Remember that the answer must contain only prime numbers. Are all the factors here prime? Students should answer "No, it's not a final answer because 6 is not a prime number; it can be decomposed."

Write $12=2 \times 6=2 \times 2 \times 3$ on the board. At this point, ask if the process of decomposition is complete. "Are all the numbers prime?" Students should say yes.

There may be some students who will ask if 12 can be represented as $4 \times 3$, instead of $2 \times 6$. Both variants are correct and decompose is the same:

$12=4 \times 3=2 \times 2 \times 3 \quad 12=2 \times 6=2 \times 2 \times 3$

It's very important to solve a number of problems like these with the students joining in the conversation. They need to master decomposition of numbers because it helps them see the structure of numbers and to solve problems that contain fractions quite easily,

It's not necessary to ask your students to memorize the definitions of a prime and a composite number because practice shows that students intuitively understand these concepts. Almost all students can tell a composite number from a prime number even if they don't remember the exact definitions. Practice is a key to success here. You'll need to solve enough problems with your students to make sure that everybody understands how to decompose 
numbers. Only after you see that your students can do it correctly and easily, can you begin to study fractions and how to cancel a common factor in a numerator and denominator.

The normal method for teaching students how to cancel a common factor is by finding the greatest common factor (GCF) of a numerator and denominator of the fraction [4]. But the author knows from experience that it's difficult for the students to memorize the algorithm of finding GCF and they often confuse GCF with LCM (the least common multiple). Actually finding GCF is not necessary to simplify a fraction. It's enough just to decompose the numerator and denominator and then to cancel all common factors.

Before discussing a concrete example, it's important to remind students that the numerator is the "top" of a fraction and the denominator is the "bottom" of a fraction in order to make the material more understandable for all students in the class.

Problem 2. Simplify this fraction into its simplest form): $\frac{20}{24}$

Discussion Strategy: The instructor should tell the students to "Decompose the numerator (the "top" of the fraction) and the denominator (the "bottom" of the fraction) writing this on the board:

$$
\frac{20}{24}=\frac{10 \cdot 2}{6 \cdot 4}
$$

You should point out that "The process of decomposition is incomplete," and ask students to continue decomposing the numbers until all factors become prime numbers. Write this on the board:

\section{$\frac{5 \cdot 2 \cdot 2}{2 \cdot 3 \cdot 2 \cdot 2}$}

At this point, you can say "Now all factors are prime and we can cancel common factors (here we can cancel 2 twice)" writing this on the board:

$$
\frac{5 \cdot 2 \cdot 2}{2 \cdot 3 \cdot 2 \cdot 2}=\frac{5}{2 \cdot 3}
$$

You can now tell the students "We've canceled all common factors so it's time to "assemble" our fraction)" writing this on the board:

$$
\frac{5}{6}
$$


This is the final answer. Since we've canceled the common factors, our fraction is in its simplest form.

Here is another problem demonstration to write on the board:

$\frac{36}{210}=\frac{4 \cdot 9}{21 \cdot 10}=\frac{2 \cdot 2 \cdot 3 \cdot 3}{3 \cdot 7 \cdot 5 \cdot 2}($ we can cancel 2 and 3$)=\frac{2 \cdot 3}{7 \cdot 5}=\frac{6}{35}$

You can see that to solve such problems, students don't really need a concept of GCF. They just need to decompose the numerator and denominator, cancel common factors and simplify the resulting fraction.

After students master this material, the lessons can move on to multiplication and division of fractions. (Usually instructors study addition and subtraction first but decomposition works perfectly for multiplication and division of fractions too. Practicing it with multiplication and division will help students gain confidence and the skills necessary for studying more difficult material (addition and subtraction of fractions).

Problem 3. Solve the following multiplication of fractions: (Write this on the board):

$\frac{18}{35} \cdot \frac{21}{32}=\frac{2 \cdot 9}{7 \cdot 5} \cdot \frac{7 \cdot 3}{8 \cdot 4}$

Remind your students that all numbers in the expression must be decomposed completely before we can cancel common factors.

$\frac{2 \cdot 3 \cdot 3}{7 \cdot 5} \cdot \frac{7 \cdot 3}{2 \cdot 2 \cdot 2 \cdot 2 \cdot 2}($ we can cancel 2 and 7$)=\frac{3 \cdot 3 \cdot 3}{5 \cdot 2 \cdot 2 \cdot 2 \cdot 2 \cdot 2}=\frac{27}{80}$.

The same principle works great for division of fractions. You just need to give your students this formula for division:

$\frac{a}{b} \div \frac{c}{d}=\frac{a}{b} \cdot \frac{d}{c}=\frac{a d}{b c}$

Using the formula,

$\frac{36}{45} \div \frac{24}{42}=\frac{36}{45} \cdot \frac{42}{24}=\frac{6 \cdot 6}{9 \cdot 5} \cdot \frac{6 \cdot 7}{8 \cdot 3}=\frac{2 \cdot 3 \cdot 2 \cdot 3}{3 \cdot 3 \cdot 5} \cdot \frac{2 \cdot 3 \cdot 7}{2 \cdot 2 \cdot 2 \cdot 3}$

Tell your students we can cancel three twos and two threes so the final answer is $\frac{7}{5}\left(=1 \frac{2}{5}\right)$.

Practice shows that students solve such problems correctly, easily, and quickly when they use the described method and they usually enjoy the process of decomposing numbers and cancelling common factors. 
Addition and subtraction of fractions are much more difficult for students.

As known, fractions can be added and/or subtracted only if they have a common denominator. If the denominators are different, the students need to find a common denominator and reduce the fractions to this denominator. As a rule, instructors tell the students that they need to find LCM (the least common multiple) of the denominators in order to find a common denominator of the fractions [4]. The algorithm of finding LCM seems difficult and complicated to many students and they often confuse it with the algorithm of finding GCF.

With this in mind, it can be more effective to use decomposition of denominators of both fractions to find the common denominator. Before solving the problems by this method the students need to be reminded that a numerator and denominator of a fraction can be multiplied by the same non-zero number without changing the value of the fraction: $\frac{a}{b}=\frac{a \cdot c}{b \cdot c}$

So the procedure of adding fractions will look like this:

Problem 4. Solve this problem: $\frac{7}{10}+\frac{1}{6}$

Discussion: Tell students "The denominators are different and we can't add the fractions right now. Let's decompose the both denominators to see their structure" and write this on the board $\frac{7}{5 \cdot 2}+\frac{1}{3 \cdot 2}$

Say to students "The denominators must be the same in order to add fractions. What is missing in the denominator of the first fraction? We have 5 and 2 there but we don't have 3. So, to get the missing number 3 in the "bottom" of the first fraction, we need to multiply the "top" and the "bottom" of the fraction by 3." Write this on the board:

\section{$\frac{7 \cdot 3}{5 \cdot 2 \cdot 3}$}

"Now, what is missing in the "bottom" of the second fraction?" Students will say "We have 3 and 2 there but we don't have 5."

Ask "How can we get 5 in there?"

Students will say, "We need to multiply the 'top' and the 'bottom' of the fraction by 5."

$$
\frac{1}{3 \cdot 2}=\frac{1 \cdot 5}{3 \cdot 2 \cdot 5}
$$


Now we have:

$$
\frac{7}{10}+\frac{1}{6}=\frac{7}{5 \cdot 2}+\frac{1}{3 \cdot 2}=\frac{7 \cdot 3}{5 \cdot 2 \cdot 3}+\frac{1 \cdot 5}{3 \cdot 2 \cdot 5} \text {. }
$$

Say to the students "Now the denominators are the same $(5 \cdot 2 \cdot 3=3 \cdot 2 \cdot 5)$, so we're in the position to add our fractions. But before doing it we need to simplify our fractions (we don't need the prime factorizations of the bottoms anymore because we've already found the common denominator):

$$
\frac{7 \cdot 3}{5 \cdot 2 \cdot 3}+\frac{1 \cdot 5}{3 \cdot 2 \cdot 5}=\frac{7}{30}+\frac{5}{30}=\frac{12}{30} \quad\left(=\frac{2}{5}\right)
$$

Problem 5. Solve this problem: $\frac{3}{16}+\frac{5}{8}$.

Discussion: tell the students "The denominators are different and we can't add the fractions right now. Let's decompose the both denominators to see their structure" and write this on the board:

$$
\frac{3}{2 \cdot 2 \cdot 2 \cdot 2}+\frac{5}{2 \cdot 2 \cdot 2}
$$

Say to the students "The denominators must be the same in order to add fractions. What is missing in the denominator of the first fraction?"

Students will say "Nothing is missing, we have four twos here."

Say to students "What is missing in the denominator of the second fraction?"

Students will say "Two is missing because in the bottom of the first fraction we have four twos and in the bottom of the second fraction we have only three twos. So we need to multiply the top and the bottom of the second fraction by the missing number 2 ".

Now we have:

$$
\frac{3}{16}+\frac{5}{8}=\frac{3}{2 \cdot 2 \cdot 2 \cdot 2}+\frac{5}{2 \cdot 2 \cdot 2}=\frac{3}{2 \cdot 2 \cdot 2 \cdot 2}+\frac{5 \cdot 2}{2 \cdot 2 \cdot 2 \cdot 2}=\frac{3}{16}+\frac{10}{16}=\frac{13}{16} .
$$

Of course I've presented only a brief description of teaching fractions by decomposition, but I hope this method will resonate with other Basic Mathematics teachers who face similar barriers to student success.

I'd like to conclude with a few suggestions for my colleagues that have worked for me. 
First, it is critical to maintain student engagement - even for those students who claim to know "all this stuff." A good solution to this problem is to make such students your helpers during a class session. For example, you can ask them to "remind" you of some useful formulas instead of writing them down by yourself. Actually every student in class can become your "helper" for solving more or less difficult math problems. Of course even the best students sometimes make errors so you should be ready to correct them if needed. It's of the utmost importance to keep your students willing to assist you during the class, to make them actively involved in the learning process. It's possible to attain this only if you are polite and friendly. When you encourage students to help and they make an error, you should never criticize them rudely and don't allow other students in class to do so. Instead, correct the student politely and at the end of the discussion thank the student for his/her help.

In accordance with the student-centered theory of teaching, maintaining a friendly, comfortable atmosphere during every class session is one of the essential factors that will allow you to succeed in teaching not only Basic Mathematics but courses in all disciplines.

\section{References}

1. Friedberg, Solomon. Teaching Mathematics in Colleges and Universities: Case Studies for Today's Classroom/Solomon Friedberg. - Student ed. Conference Board of Mathematical Sciences, Rhode Island, 2001

2. Kelton, Suzanne. An Introduction to Teaching Mathematics at the College Level/ Suzanne Kelton - Assumption College (https://www.ams.org/profession/careerinfo/grad-school/Kelton-TEACH.pdf)

3. Krantz, Steven. How to Teach Mathematics/Steven G. Krantz - $3^{\text {rd }}$ edition. American Mathematical Society, Rhode Island, 2015, P. 26.

4. Aufman, Richard and Lockwood, Joanne. Introductory Algebra: an Applied Approach, 2011 
DOI 10.32370/IA_2020_01_14

\title{
TERMINOLOGY WORK OF FUTURE BIOLOGY TEACHERS DURING THE FIELD-BASED TRAINING IN BOTANY
}

Pererva Viktoriia

\author{
Assistant of Botany and Ecology Department of Kryvyi Rih State Pedagogical \\ University, Kryvyi Rih, Ukraine
}

ORCID ID 0000-0002-7086-3050 pererva@kdpu.edu.ua

\begin{abstract}
This paper focuses on problems of terminology work during the field-based training in botany and examines means and exercises of forming professional and terminological competence of future biology teachers. It is shown that improving the level of professional and terminological competence of students provides the background to better scientific awareness of students and contributes to improvement of the quality of academic activity during the professional practice.

Biology is based on the results of field research and discoveries. Therefore training that occurs in a field setting is a powerful experience that promotes the development of creative teachers, enhances environmental literacy, and instills ecological responsibility. The content literacy is an important component of studies. In order to improve literacy, it is primarily necessary to form an active professional vocabulary.
\end{abstract}

Keywords: terminology, professional and terminological competence, field-based training in botany, future biology teacher.

\section{Introduction}

The main guideline of modern higher education is the search for methodological ways of organizing the educational process of training a competent specialist. The future biology teacher should optimally organize his professional and communicative activities. A basis for this is proficiency in professional terminology, which is one of the indicators of understanding professional and subject concepts, the level of culture of professional communication.

That is why mastering professional terminology is crucial to the formation of professional and terminological competence of the future specialist and one of the important problems of modern professional education.

The relevance of this study is due to the lack of experience that would identify the specificity and system of terminology work during the field-based training in botany.

\section{Statement of the problem}

Scientific terminology is the highest manifestation of human thought, the basic structural element of improving and standardizing the language of a particular industry. 
Terminology is a general word for the group of specialized words or meanings relating to a particular field, and also the study of such terms and their use [9]. Terms are words and compound words or multi-word expressions that in specific contexts are given specific meanings - these may deviate from the meanings the same words have in other contexts and in everyday language [6]. A definition is a statement of the meaning of a term (a word, phrase, or other set of symbols). Terms are used in the knowledge and assimilation of scientific and professional objects and relations between them.

In the analysis of terms, the priority is given to the structural and cognitive aspect, mastering the term elements. Since biological terminology has Latin origin, we consider it necessary to study the semantics and the etymology of the terminological elements of Greek and Latin origin.

An individual active terminological vocabulary of a future specialist is the basis of his professional and terminological competence. It is formed as a result of mastering the subordinated system of term elements.

Professional and terminological competence is a formed capacity to use professional terms correctly and precisely during the professional activity; acquired skills of oral and written speech with use of experience gained during the studies of professional disciplines.

The purpose of the article is to reveal the essence, conditions and specifics of the terminology work of future biology teachers during the field-based training in botany.

The purpose is addressing two tasks concerning the field-based training in biology (botany): 1. The role of field-based experience is important to consolidation of biological terminology. 2. How the fieldwork may enhance the students proficiency in biological terminology. Here, we explore each of these questions and offer suggestions about the ways to ensure that future generations of biologists will be able to master the terminology knowledge that can be acquired in field settings.

\section{Summary of the Research}

The professional activity of a teacher needs both professional mastery and wide general knowledge of subject (professional competence) during the oral and written communication. Modern higher school is aimed more at the formation of creative abilities than just developing mental skills of the future specialist. The ability to use some acquired knowledge in a creative way represents social value since it helps the specialist to perform his social and professional 
functions, to adapt quickly to new economic conditions, contributes to a flexible transition from one activity to another. Professional competence is determined by the correct application of professional terms in the professional activity.

Field-based education is particularly critical to the biological sciences, providing fundamental training for key disciplines such as botany, zoology, ecology, physiology, systematics, and conservation science $[1,4,11]$. Field studies underlie the conceptual and technical bases for these disciplines and are required to ensure their healthy growth. Now, as society struggles to respond appropriately to losses of biodiversity, range shifts due to climate change, and the emergence of new human pathogens, the decline in opportunities for field study means that subsequent generations of biologists will be increasingly divorced from the primary setting, the natural environment, in which the phenomena that they study occur. As the capacity to modify biological systems expands from genomes to ecosystems to global cycles, it is imperative that scientists and the broader public are able to critically evaluate the outcomes of these changes in the context of complex natural settings. Within academia, this need also applies to the educators charged with training future generations of problem-solvers [7]. In summary, field studies are an essential component of every scientist's training [4].

Fieldwork tasks should be as productive as possible and recognize ways in which the learning experience could be further enhanced [2]. Enhancements range from a staged sequence of enquiry-based exercises [5] through use of mobile devices to utilise travel time effectively $[1,3]$

The field practice is a very important practice link in pharmacy undergraduate education, and it is an important teaching link of botany theory [12].

The field-based training is a type of practical and research activity that is important during the professional training of students for their future professional activity, including environmental education of schoolchildren. The program in biology for a secondary school involves teaching the elements of research work with plants and educational excursions. Thus a biology teacher must have necessary skills of arrangement and conducting excursions in nature, research and practical work, i.e. he/she must be professionally competent.

In this paper we propose to analyse the conditions and methods of formation of terminological competence of biology students during the field-based training in botany, the section «Anatomy and morphology of plants». Its goal is to form the skills to distinguish plants 
in nature and describe them as organisms; to master a field method of research and a laboratory one for studying streptophyta; to study species composition of plants in Dnipropetrovsk region; to reveal anatomical and morphological peculiarities of plants conditioned by the mutual influence of organisms (symbiotic, competitive, parasitic, allelopathic) and their interaction with non-living components of environment (morphological peculiarities of plants being a part of different ecological groups); promotion of activities aimed at the environmental protection.

The main tasks of the practice are consolidation and broadening of knowledge about the anatomical and morphological peculiarities of plants of different localities; mastering the techniques of collecting plant specimens and making herbaria, compiling systematic and biological collections; forming the skills to carry out excursions in different biotopes (steppe areas, forest plantations, meadows, agrophytocenoses, coastal and anthropogenically transformed areas, etc.); formation of a caring attitude to the environment, studying rare and endangered species of local flora [8].

During the field-based training students study species composition of flora, zonal and anthropogenically changed vegetation, carry out phenological observations, reveal biological and ecological peculiarities and distribution of different plant species, work out the methods of morphological analysis and identification of plants as well as the methods of collecting, fixation and laboratory treatment of field data.

The summer field-based training in anatomy and morphology of plants aims to systematize and consolidate students' knowledge in botany (including understanding and proper use of botanic terminology); to acquire the methods of collecting of algal and mycological material in nature, making herbaria of vascular plants; to teach students to make morphological and biological descriptions of woody and herbaceous plants of local flora, to determine their systematic position according to the identification guide; to apply the skills of carrying out geobotanical descriptions of a plot (formation of skills to carry out a research in nature).

The field-based training ensures actualization, systematization and consolidation of the ecological and botanical term system concerning the anatomical (tissue level of organization) and morphological (organ level of organization) plant structure, the specificity of anatomical and morphological structure of plants being a part of different ecological groups depending on the humidity level (hygromorphic plants), soil condition (trophomorph species), soil texture, 
temperature (thermomorphs, climamorphs), correlation with a certain type of phytocenosis (cenomorphs) etc.

It should be noted that knowledge and skills acquired and consolidated during the summer field-based training represent the basis for the further learning of academic subjects of fundamental (natural science) and scientific training.

I. Early spring excursions are held in April or May and they are timed to the study of ephemerals and ephemeroids, phenological observations in nature at the beginning of the plants vegetation. They are usually carried out in park areas or a botanical garden after classes. During this type of excursions students work out ecological and botanical term system related to prevernal vegetation, life cycle and life forms of plants: ephemerals, ephemeroids, underground modifications of shoots (tubers, bulbs, rhizomes, corms), rosellate shoots, vegetation, juvenile, verginile, generative and senile stages of plants development, budding and flowering, an inflorescence, a habitus, a herbaceous plant, a shrub, a subshrub, a dwarf shrub, a dwarf subshrub, a tree, vegetative and generative buds, catkins, pollinosis etc.

The summer field-based training in botany includes some excursions in nature (in June) to different biotopes: steppe areas, meadows, coastal areas, man-made forest plantings (forest belts and park areas), anthropogenically transformed areas, agrophytocenoses and conservation areas. During these excursions students have an opportunity to study the species diversity of vegetation communities in the biotopes mentioned above and peculiarities of the anatomical and morphological plant structure that ensure plant adaptations to different growth conditions.

II. The study of steppe vegetation. The zonal type of vegetation in the Dnipropetrovsk region is the steppe, but it has been preserved in its original form only in locations inaccessible to farming or on the conservation areas. As a result students can learn the main representatives of steppe flora during the excursion to Kryvyi Rih Botanical Garden of the National Academy of Sciences of Ukraine (exposition of steppe vegetation) and the excursion to the nature monument «Rocks of MODR». During these excursions the students work out some notions referring to ecological and biological system, for example: zonal vegetation, climax communities, steppe plants, sclerophytes, succulent plants, heliophytes, psammophytes, petrophytes, halophytes, arid climate etc.

III. The study of meadow vegetation. The students can learn about meadow and coastal vegetation during the excursions to the lake Solone, the ponds on «Terminal» and the 
Karachunovsky reservoir. This kind of excursions is related to mastering such terms of ecological and biological system as meadows, meadow vegetation, coastal vegetation, paludant , eumesophyte, mesophyte, xeromesophyte and mesoxerophyte, hygrophyte, heliophyte, sciophyte.

IV. The study of agrophytocenoses. Excursions to agrophytocenoses are necessary for revealing the functional peculiarities and supporting the monocultural growth of plants; functioning of climax multi-species zonal communities. There the students practise using such terms of ecological and biological system as phytocenosis, plant community, biocoenosis, biogeocenosis, monoculture, cultivated plant, weed, ruderal and segetal vegetation, expansion, vital force, competitors, stress tolerators and ruderals etc.

V. The study of anthropogenically transformed areas. The aim of excursions carried out in such kind of biotopes is to reveal the specific features of anthropogenic changes of natural zonal vegetation. So far as Kryvyi Rih is a city with developed mining and metallurgical industry, its basis is represented exactly by ecotopes of anthropogenic type. There are some restrictions for carrying out training excursions on industrial territories, but we have access to adjacent areas that also have a high anthropogenic load. It is possible to study the vegetation in sanitary-hygienic zone (forest plantings) of ore-dressing enterprises. The main goal of these field studies is application and consolidation of terms concerning anthropogenic flora, e.g.: melioration plant, sanitation, ruderants, plants of residential area, anthropogenic transformation, anthropogenic load, vegetational fluctuation and its types, etc.

It is obvious that during the excursions the students constantly revise and use their knowledge of anatomy and morphology of vegetative and generative parts of plants, adaptive peculiarities in the morphology of these parts, vital forms, ecological groups of plants, types of reproduction and habitat of plants paying attention to the following aspects:

1. Non-vascular plants (also known as thallophytes or 'lower plants') and fungi: ecological groups of non-vascular plants, their value in biocenosis; morphological features of the sporocarp or fruiting body structure of different fungi classes and genera, a type of their nutrition;

2. Vascular plants (also known as streptophyta or 'higher plants'): plant propagation and plant life-forms; differences in the structure of sporous and seed plants of different systematic groups (alternation of sporophyte and gametophyte generations); structure of seeds and 
seedlings (comparative analysis of monocotyledonous (monocots) and dicotyledonous (dicots) plants); morphology of vegetative organs of plants such as a root, a shoot, modified stems; structure of plan generative organs (morphology of the gynoecium, the androecium, the perianth, racemose (monopodial) and cymose (sympodial) inflorescences); structure of fruits (dry and fleshy, simple, aggregate and multiple or collective fruits).

\section{Conclusions and prospects for further research}

All things considered we can come to the conclusion that the formation of students' terminological and professional competence is realized when they learn professional academic disciplines. The formation and improvement of the professional and terminological competence of future biology teachers in their professional field is achieved by forming the motivation to independent work aiming the improvement of professional communication and implementation of specially developed methodology.

The experience of educational and practical activity of biology students has shown that much attention is paid to the revealing of plants adaptation to growth conditions, to issues of protection and reproduction of flora, to actualisation and consolidation of theoretical knowledge acquiered during the study of the discipline «Botany with the basics of geobotany», to mastering practical skills of plants study in nature. The future teacher will be able to realize these practical skills of field research when he works with students at school during excursions in nature, optional classes, extracurricular and scientific work (managing students' scientific research). So it is obvious that the field-based learning in botany contributes to the consolidation of theoretical knowledge and enables the formation of practical skills in various field conditions, provides the ecological approach to learning, and it is an important stage of training highly qualified specialists in the system of modern pedagogical education.

\section{References}

[1] Baggott, G.K. \& Rayne, R.C. 2007. The use of computer-based assessments in a field biology module. Bioscience Education e-Journal, 9, (art. 5). Retrieved from https://eprints.bbk.ac.uk/538/1/beej-9-5.pdf (accessed 15 January 2020).

[2] Besenyei, L., Watkin, G. \& Oliver, K. 2003. An evaluation of the educational effectiveness of fieldwork within environmental science awards at the University of 
Wolverhampton. CELT Learning and Teaching Projects. Retrieved from http://hdl.handle.net/2436/3694 (accessed 15 January 2020).

[3] Elkins, J.T. \& Elkins, N.M.L. 2006. Improving Student Learning During Travel Time on Field Trips Using an Innovative, Portable Audio/video System. Journal of Geoscience Education, 54 (2), 147-152.

[4] Fleischner, T., Espinoza, R., Gerrish, G. et al. 2017. Teaching Biology in the Field: Importance, Challenges, and Solutions. BioScience, Volume 67, Issue 6, P.558-567. DOI: $\quad 10.1093 /$ biosci/bix036 Retrieved from https://academic.oup.com/bioscience/article /67/6/558/3798229 (accessed 15 January 2020).

[5] Fleischner, T.L. 2005. Natural history and the deep roots of resource management. Atural Resources Journal, 45: 1-13.

[6] Merriam-Webster. 2015. Dictionary. Encyclopedia Britannica Online. Retrieved from https://www.merriam-webster.com/dictionary/dictionary (accessed 15 January 2020).

[7] Pauly, D. 1995. Anecdotes and the shifting baseline syndrome of fisheries. Trends in Ecology and Evolution, 10: 430.

[8] Pererva, V. V. 2018. Training Field Practice as Means of Shaping of Professional Competence the Future Biology Teachers. Adaptive Management: Theory and $\begin{array}{llll}\text { Practice. } & \text { Pedagogics, } & \text { Retrieved } & \text { from }\end{array}$ https://amtp.org.ua/index.php/journal/article/view/112 (accessed 15 January 2020).

[9] Sonneveld, H. \& Loenning, K. 1994. Introducing terminology, in Terminology, p. 1-6.

[10] Tingting, D., Jin, L. \& Chao, Z. 2019. Teaching Reforms of Field Practice in Botany. 9th International Conference on Management, Education and Information (MEICI 2019). Retrieved from https://webofproceedings.org/proceedings_series /ESSP/MEICI\%202019/MEICI19086.pdf (accessed 15 January 2020).

[11] Wilson, E.O. 1982. The importance of biological field stations. BioScience, 32: 320. DOI: $10.2307 / 1308847$.

[12] Wu, J., Guo, Y. \& Ping, W. 2016. The teaching reform of Pharmaceutical Botany Field Practice in teaching mode of competence-based. Advances in Social Science, Education and Humanities Research, volume 87 272. 2nd International Conference on Economics, Management Engineering and Education Technology (ICEMEET 2016). 


\title{
Retrieved from https://www.atlantis-press.com/proceedings/icemeet-16/25869116
} (accessed 15 January 2020).

\begin{abstract}
Анотація
У роботі проаналізовано проблеми термінологічної роботи під час навчально-польової практики з ботаніки та розглянуто засоби та послідовність формування професійно-термінологічної компетентності майбутніх учителів біології. Доведено, що підвищення рівня професійно-термінологічної компетентності студентів створює передумови зростання наукової обізнаності студентів та покращення якості навчальної діяльності під час фахової практики.

Біологія базується на результатах польових досліджень та лабораторних відкриттів. Тому навчання у польових умовах надає суттєві практичні знання, що сприяють розвитку творчості вчителів, підвищують екологічну грамотність та екологічну відповідальність. Важливою складовою навчання $\epsilon$ змістова грамотність. Для підвищення рівня грамотності, передусім необхідним є формування активного фахового словника.
\end{abstract}

Ключові слова: термінологія, професійно-термінологічна компетентність, навчально-польова практика з ботаніки, майбутній вчитель біології.

\section{Аннотация}

В работе представлена проблема терминологической работы во время ботанической учебнополевой практики, рассмотрены средства и последовательность формирования профессиональнотерминологической компетентности будущих учителей биологии. Доказано, что повышение уровня профессионально-терминологической компетентности студентов создает предпосылки роста научной осведомленности студентов и улучшения качества учебной деятельности во время профессиональной практики.

Биологические знания базируются на результатах полевых исследований и лабораторных открытий. Поэтому обучение в полевых условиях дает существенные практические знания, которые способствуют развитию творчества учителей, повышают экологическую грамотность и экологическую ответственность. Важной составляющей обучения является содержательная грамотность. Для повышения уровня грамотности определяющим условием является формирования активного профессионального словаря.

Ключвые слова: терминология, профессионально-терминологическая компетентность, учебно-полевая практика с ботаники, будущий учитель биологии. 


\title{
Diagnostics of Formation the Subject Competences in Future Doctors
}

\author{
Khmil Iryna \\ Senior Lecturer \\ Bogomolets National Medical University (Ukraine, Kyiv)
}

\begin{abstract}
The article analyzes the results of diagnostics of future doctors' motivational-value sphere. The peculiarities of studying the significance of motives in forming subject competences of students are revealed. It is stated that the greatest value for students is the external motives, which reflect the material interest of the individual in the results of their professional activity. In the hierarchy of students' motives, respectively, the following rankings are occupied by motives of prestige, motives of manifestation of personality in the profession and professional motives. The motives of their professional activity characterize the students' interest in the profession of doctor. Proved that the motives of the professional activity itself constitute a valuable basis for the content of the profession.

Keywords: future doctors, student, subject competence, motives, value-motivational sphere, diagnostics, methodology, surveys.
\end{abstract}

Постановка проблеми. В сучасних соціально-економічних умовах функціонування системи охорони здоров'я суттєво зростають вимоги до рівня правової підготовки майбутніх лікарів. Правова підготовка майбутніх лікарів $є$ базовою системотвірною ланкою у формуванні їхніх професійних знань та умінь. Разом з тим спостерігаються протиріччя між запровадженням сучасних форм, методів формування предметних компетентностей у процесі вивчення медичного правознавства та недостатнім науково-методичним забезпеченням, моніторингом цього процесу у закладах вищої медичної освіти, низьким рівнем мотивації студентів. Означені протиріччя потребують негайного вирішення.

Метою статті $є$ діагностика у студентів мотивів вступу в університет, мотивів вивчення медичного правознавства.

Аналіз попередніх досліджень і публікацій. Питання формування предметних компетентностей у своїх працях досліджували А. Будас, Л. Овсієнко, Н. Писименна, О. Пишко та ін. Правова компетентність майбутніх фахівців була предметом вивчення А. Анікіної, С. Воєводіної, Т. Волох, С. Гуріна, Я. Кічук, І. Огороднійчук, О. Панова, М. Полякової та ін. Підготовку фахівців медичної галузі висвітлено у дослідженнях 
М. Асмолова, М. Банчук, О. Волосовець, Л. Войтенко, В. Лазоришенець, М. Мруги, Н. Стучинської, Т. Реви, І. Фещенко, Я. Цехмістера, О. Чалого, Б. Шахова та ін.

Водночас аналіз психолого-педагогічної літератури свідчить про недостатню розробку проблеми формування предметних компетентностей майбутніх лікарів, зокрема в контексті вивчення впливу мотивації на означений процес.

Результати дослідження. Предметну правову компетентність розглядаємо як сукупність набутих майбутнім лікарем знань 3 медичного правознавства, умінь екстраполювати їх у практичну діяльність, здатності розв'язувати пізнавальні завдання правового змісту в освітньому процесі та життєвих ситуаціях. Предметну компетентність з медичного правознавства характеризуємо як показник високої якості їх правових знань, умінь, здатності установлювати зв'язки між набутими знаннями та реальними практичними ситуаціями.

Вивчення рівнів сформованості предметних компетентностей майбутніх лікарів передбачало визначення наявності у студентів провідних ціннісних мотивів вступу до університету, мотивів вивчення медичного правознавства.

Для дослідження мотивів вступу до магістратури студентам було запропоновано анкету, розроблену на основі методики Т. Ільїної «Мотивація навчання у ЗВО». Виявлення провідних мотивів вступу студентів до магістратури здійснювали за такими шкалами: 1) отримання знань (прагнення до отримання знань, допитливість); 2) оволодіння професією (прагнення до оволодіння професійними знаннями, правовими уміннями розвитку професійно важливих якостей); 3) отримання диплому (прагнення до отримання диплому за умови формального засвоєння навчальних предметів, іноді пошук неетичних, незаконних засобів для отримання заліків та оцінки на іспитах).

Результати опитування засвідчують, що більшість студентів надали перевагу мотиву «отримання диплому» - 52,8 \%, мотив «отримання знань» зайняв друге місце, його обрали - 33,9\% респондентів. Мотивом «оволодіння професією» керувались у своєму виборі закладу вищої освіти тільки 13,3 \% майбутніх лікарів. Відтак можемо констатувати, що провідним мотивом навчальної діяльності студентів у даній вибірці виявився мотив «отримання диплому», що ще раз підтверджує низьку мотивацію до оволодіння професією та низький рівень сформованості цінностей, пов'язаних із нею.

Слід зазначити, що серед провідних мотивів навчання майбутніх лікарів мотив 
«отримання диплому» займає перше місце. Отриманий результат можна пояснити, на наш погляд, з одного боку - прагненням підтвердити свою дорослість і незалежність, а з іншого - недостатнім розвитком пізнавального інтересу у певної частини студентів. Мотив «отримання знань» у переважної кількості студентів виражений досить посередньо, мотивація до здобуття знань дещо занижена. Серед мотивів оволодіння професією в обох групах переважають зовнішні мотиви, значущим внутрішнім мотивом є лише позитивне ставлення до певних навчальних дисциплін, які вивчаються в університеті. Можна стверджувати, що ціннісні орієнтації значної частини студентів лежать поза межами професії лікаря. Отже, формування перших двох показників предметних компетентностей («отримання знань» та «оволодіння професією») потребують особливої уваги в процесі підготовки майбутніх лікарів.

Так, рушійною силою будь-якої діяльності, зокрема щодо формування предметних компетентностей, вивчення медичного правознавства $є$ мотивація. Ми вважали за необхідне детальніше дослідити ціннісно-мотиваційну сферу майбутніх лікарів, можливості впливу на неї, також нас цікавили питання організації освітнього процесу (вплив знань і вмінь студентів на процес і результат оволодіння предметними компетентностями 3 медичного правознавства). Усе це обумовило необхідність вивчення значущості мотивів формування предметних компетентностей у майбутніх лікарів у зазначених групах. 3 метою вивчення значущості мотивів нами використовувалася методика самооцінки мотивів, запропонована М. Віленським, П. Образцовим, А. Уманом [3].

Кількість мотивів визначалася необхідністю вибору студентами найбільш значущих для них мотивів, відповідних їх суб'єктивній ранговій системі цінностей, цілей. Результати оцінювання значущості мотивів, які спонукають майбутніх лікарів до формування правової предметної компетентності у процесі навчання медичного правознавства засвідчує, що найбільшу цінність для студентів мають зовнішні мотиви, що відображають матеріальну зацікавленість особистості в результатах своєї професійної діяльності: бажання забезпечити високий заробіток завдяки отриманим знанням, отримати високооплачувану роботу. Отже, престижність на ринку праці, яка асоціюється з їі затребуваністю бачать 16,8 \% студентів та як наслідок, матеріальне благополуччя, пов'язане із професією 29,7 \%. Зовнішній мотив займає чільне місце в 
мотивації навчальної діяльності, але не є визначальним (скоріше за все, він пов'язаний 3 віддаленою перспективою заробляти гроші, а навчальна діяльність розглядається як засіб досягнення цієї мети).

Мотиви престижу професії, які тісно пов'язані із прагненням особистості до самоствердження у суспільстві займають друге рангове місце в ієрархії мотивів навчання. Вища освіта багатьма молодими людьми автоматично сприймається як універсальна цінність, абсолютно логічний етап власного соціального становлення. Поряд із цим тут присутня й статусна мотивація. Для успішного проходження конкурсу, формування корпоративної культури, ефективного засвоєння медичного правознавства необхідний досить високий рівень домагань особистості та інших суб'єктивних факторів. Одержання статусу магістра, за результатами наших досліджень, дотепер сприймається як високий рівень саморозвитку в неформальній молодіжній ієрархії, досягнення значимої чергової (після закінчення бакалаврату) соціально-ієрархічної сходинки. 3 огляду на це, можна констатувати, що сучасні магістри $[4 ; 6 ; 7 ; 10 ; 11 ; 11$; $12 ; 16 ; 17 ; 19]$ недостатньою мірою орієнтовані на навчання заради формування власної громадянської позиції, розбудови країни.

Наступне місце в ієрархії мотивів навчання займають мотиви прояву особистості у професії, що супроводжується особливостями формування самосвідомості особистості в умовах взаємодії з професією (переконаність у власній професійній придатності, у достатньому творчому потенціалі, в тому, що обрана професія $\epsilon$ покликанням та ін.). Ці студенти займають активну соціальну й пізнавальну позицію. Навчання, формування предметних компетентностей відбувається у процесі реалізації власного інтелектуального та особистісного потенціалу. Професійна самореалізація майбутніх лікарів включає пошук «себе в професії», формування професійного іміджу, власного образу «Я», окреслення для себе професійних перспектив та шляхів їх досягнення, побудова своєї діяльності на правовій основі. Їх дії спрямовані на розуміння ступеня відповідності індивідуальних особливостей вимогам професії, яку вони обрали; на розвиток власних можливостей в процесі навчання медичного правознавства. Сформованість такої мотивації $є$ соціально значущою та впливає на результат майбутньої професійної діяльності. 
Важливе місце в мотиваційній структурі навчальної діяльності майбутніх лікарів посів мотив розуміння призначення професії. Мотив розуміння призначення професії формується як інтерес до професії та схильність займатися нею. В ідеалі мотиви розуміння призначення професії повинні збігатися з мотивами вибору. Важливо щоб майбутній лікар був готовий до вирішення професійних завдань, виявляв інтерес до професії та ціннісне ставлення до неї. Так, мотиви розуміння призначення професії відображають також бажання відповідати образу професії й орієнтують на розвиток таких важливих для фахівця професійних якостей, як комунікабельність, уважність, гнучкість, толерантність, емпатія, відповідальність, рефлексія щодо власних вчинків і рішень, порядність, дотримання правових та етичних норм у майбутній професійній діяльності, неприпустимість маніпулювання людьми.

До власне професійних мотивів відносимо мотиви, пов'язані з усвідомленням соціальної та особистісної значущості професійної діяльності лікаря, що підкріплюються задоволеністю результатами самої діяльності (стати висококваліфікованим спеціалістом, забезпечити успішність майбутньої професійної діяльності, домогтися високих результатів діяльності у сфері медицини та ін). Мотиви власне професійної діяльності характеризуються наявністю у студентів інтересу до професії лікаря, прагненням до досягнення успіху в реалізації поставлених цілей. Ця група мотивів пов'язана з такими особистісними якостями, як наполегливість у досягненні кінцевого результату діяльності. Вони складають ціннісну основу змісту професії.

Висновки. Отже, можна стверджувати, що не всі студенти досить усвідомлено підходять до вибору професії, більшість із них не пов'язує майбутню професійну діяльність із правовою підготовкою, знаннями й дотриманням правових норм у професійних взаєминах. Необхідна спеціальна робота щодо розвитку мотивації студентів. Подальшого дослідження потребують такі важливі аспекти цієї проблеми: застосування сучасних інформаційних технологій для формування предметних компетентностей майбутніх лікарів, дистанційна освіта у професійному розвитку лікарів.

\section{References}

1. Bakulina O.S. Suchasni problemy motyvatsii personalu silskohospodarskykh pidpryiemstv [Current problems of motivation of staff of agricultural enterprises]. Rol nauky $u$ 
pidvyshchenni tekhnolohichnoho rivnia i efektyvnosti APK Ukrainy: mater. 2-yi vseukr. nauk.prakt. konf. z mizhnar. uchastiu, (16-18 travnia 2012). Ternopil : Krok, 2012. P. 236-238.

2. Bakulina O.S. Motyvatsiia personalu silskohospodarskykh pidpryiemstv: teoretychnyi aspect [Motivation of staff of agricultural enterprises: theoretical aspect]. Visn. nats. un-tu vodnoho hospodarstva ta pryrodokorystuvannia. 2008. Issue 4(44). P. 3-10.

3. Vilenskij M. Ya. Tehnologii professionalno-orientirovannogo obucheniya $v$ vysshej shkole [Technologies of vocational-oriented education in higher education]. Moskva : Pedagogicheskoe obshestvo Rossii, 2002. 275 p.

4. Ovsiienko L. M. Problemy vprovadzhennia kompetentnisnoho pidkhodu v protses pidhotovky maibutnikh pedahohichnykh kadriv [Implications of implementing the competence-based approach into the process of professional training of future teaches]. Ukrainska mova i literatura $\mathrm{v}$ shkoli: scientific-andmethodological journal. Kyiv: Vydavnytstvo TOV «Lazuryt-Polihraf» Publ., 2011. № 6. Pp. 46-48.

5. Petko S. M. Modeli korporatyvnoho upravlinnia v diialnosti korporatsii na svitovykh rynkakh [Models of Corporate Governance in Corporate Activity of Global Markets]. Molodyi vchenyi. 2015. № 2 (17). Part 2. Pp. 225-229.

6. Pet'ko L.V. Vyklyky XXI stolittia dlia osvitnoho prostoru Ukrainy. Naukovi pratsi ChNU: nauk. zhurnal [The challenges of educational space in the $21-^{\text {st }}$ century] / Chornom. Nats. un-t im. Petra Mohyly; red. Kol.: O.P.Meshchaninov (holova) [ta in.]. - Mykolaiv: Vydvo ChNU imeni Petra Mohyly, 2017. T. 303. Issue 291. P. 10-14 (Pedahohika)

7. Pet'ko L V. Realizatsiia kontseptsii pidhotovky mahistriv $v$ Ukraini dlia roboty $v$ inkliuzyvnomu osvitnomu prostori [The conception of the preparation masters' in Ukraine Implementation to work in an inclusive educational environment] // Aktualni problemy navchannia ta vykhovannia liudei $\mathrm{v}$ intehrovanomu osvitnomu seredovyshchi: $\mathrm{Tr}$. KhI Mizhnar. nauk.-prakt. konf., lystopad 2011 r. Ch. I. Kyiv: VMURL «Ukraina». 2011. P.92-94.

8. Ternopilska V. I. Vidpovidalnist osobystosti: humanitarnyi aspect [Personality responsibility: the humanitarian aspect]. Visnyk Zhytomyr. derzh. un-tu im. I. Franka. 2004. No. 14. P. 47-50.

9. Ternopilska V. I. Teoretychni zasady realizatsii tsinnisnoho pidkhodu u vykhovanni osobystosti [Theoretical bases of realization of value approach in education of personality']. Novi tekhnolohii navchannia. 2016. Issue. 88. P. 118-121.

10. Ternopilska V. I. Suchasni tendentsii motyvatsii navchalno-profesiinoi diialnosti studentiv [Modern tendencies of motivation of students' educational and professional activity]. Visnyk Natsionalnoho universytetu oborony Ukrainy, 2012. 5(30). P. 141-144.

11. Ternopol'skaja V. I., Bakulina O. S. Osobennosti formirovanija professional'noj kompetentnosti budushhih specialistov po nalogooblozheniju i tamozhennomu delu [Features of the formation of professional competence of future specialists in taxation and customs]. Mezhdunarodnyj nauchnyj zhurnal «Progress». Tbilisi : «Progress», 2018. No. 1-2. P. 94-97.

12. Ternopilska V. I. Struktura profesiinoi kompetentnosti maibutnoho fakhivtsia [The structure of professional competence of the future specialist]. Naukovyi visnyk Melitopolskoho derzhavnoho pedahohichnoho universytetu, 2012. No. 9. P. 208-213. 
13. Ternopilska V. I., Chyzhova N. V. Mobilnist osobystosti yak predmet naukovoho doslidzhennia [Personality mobility as a subject of scientific research]. Teoretyko-metodychni problemy vykhovannia ditei ta uchnivskoi molodi. 2016. Issue 20. P. 234-243.

14. Ternopilska V. I., Derev'ianko O. V. Vyznachennia kryteriiv sformovanosti profesiinoi kompetentnosti maibutnikh hirnychykh inzheneriv [Determination of criteria for the formation of future mining engineers'professional competence]. Nauk. chasopys NPU imeni M.P. Drahomanova. Ped. nauky, 2012. Issue 31. P. 264-267.

15. Ternopilska V. I., Kolomiiets T. V., Piontkivska I. O. Dovidnyk $z$ vykhovnoi roboty zi studentamy [Handbook of educational work with students]. Ternopil : Vyd-vo «Bohdan», 2014. $184 \mathrm{p}$.

16. Babiy S., Bezkorovaina O., Matviienko O., Petko St., Ternopilska V., Soichuk R., Stanislavchuk N. Entrepreneurship model of professional development of actuaries in Canada // Journal of Entrepreneurship Education (JEI). USA. 2019. Vol: 22 Issue: 3. P. 1-6.

17. Ovsiienko Liudmyla. Innovative approaches to improving professional training of spesialists in higher educational institutions // Current scientific research: Collection of scientific articles. - Publishing house «BREEZE», Montreal, Canada, 2017. P. 215-220.

18. Ovsiienko L. M. Regularities of competently directed learning of text linguistics of studets' philological specialities // Economics, management, law: socio-economical aspects of development: Collection of scientific articles. Volum 2. Psychology. Pedagogy and Education. - Edizioni Magi - Roma, Italy. 2016. P. 245-250.

19. Pet'ko L. The development of student youth aesthetic culture on professional direction // Topical issues of contemporary science: Collection of scientific articles. - C.E.I.M., Valencia, Venezuela, 2017. P. 188-192.

Translation of the Title, Abstract and References to the Author's Language

УДК 378.147-057.875[34:61]616-051:005.336.2

Хміль І. Ю. Діагностика сформованості предметних компетентностей майбутніх лікарів.

Здійснено аналіз результатів діагностики мотиваційно-ціннісної сфери майбутніх лікарів. Розкрито особливості вивчення значущості мотивів формування предметних компетентностей студентів. Зазначено, що найбільшу цінність для студентів мають зовнішні мотиви, що відображають матеріальну зацікавленість особистості в результатах своєї професійної діяльності. В ієрархії мотивів студентів відповідно наступні рангові місця займають мотиви престижу, мотиви прояву особистості в професії та власне професійні мотиви. Мотиви власне професійної діяльності характеризують наявність у студентів інтересу до професії лікаря, прагненням до досягнення успіху в реалізації поставлених цілей. Доведено, що мотиви власне професійної діяльності складають ціннісну основу змісту професії.

Ключові слова: майбутні лікарі, студент, предметна компетентність, мотиви, ціннісно-мотиваційна сфера, діагностика, методика, опитування. 


\section{Література}

1. Бакуліна О. С. Сучасні проблеми мотивації персоналу сільськогосподарських підприємств. Роль науки у підвищенні технологічного рівня $і$ ефективності АПК України: матеріали 2-ї всеукр. наук.-практ. конф. з міжнар. Участю (м. Тернопіль, 16-18 травня 2012 р.). Тернопіль : Крок, 2012. С. 236-238.

2. Бакуліна О. С. Мотивація персоналу сільськогосподарських підприємств: теоретичний аспект. Вісн. наи. ун-ту водного господарства та природокористування. 2008. Вип. 4 (44). Ч. 5. С. 3-10.

3. Виленский М. Я. Технологии профессионально-ориентированного обучения в высшей школе: уч. пос. / под ред. В.А. Сластенина. Москва: Педагогическое общество России, 2002. 275 с.

4. Овсієнко Л. М. Проблеми впровадження компетентнісного підходу в процес підготовки майбутніх педагогічних кадрів. Украӥнська мова $і$ література в школі: науково-методичний журнал. Київ: Видавництво ТОВ «Лазурит-Поліграф», 2011. № 6. C. 46-48.

5. Петько С. М. Моделі корпоративного управління в діяльності корпорацій на світових ринках. Молодий вчений. 2015. №2 (17). Ч. 2. С. 225-229.

6. Петько Л. В. Виклики XXI століття для освітнього простору України. Наукові nраиі [Чорноморського державного університету імені Петра Могили комплексу "КиєвоМогилянська академія"]. Серія : Педагогіка : наук. журн. / Чорном. держ. ун-т імені Петра Могили; ред. кол. : О. П. Мещанінов (голова) [та ін.]. Миколаїв : Вид-во ЧНУ імені Петра Могили, 2017. Т. 303. Вип. 291. С. 10-14.

7. Петько Л В. Реалізація концепції підготовки магістрів в Україні для роботи в інклюзивному освітньому просторі // Актуальні проблеми навчання та виховання людей в інтегрованому освітньому середовищі: Тр. XI Міжнар. наук.-практ. конф., листопад 2011 р. Ч. І. Київ: ВМУРЛ «Україна». 2011. С. 92-94.

8. Тернопільська В. І. Відповідальність особистості: гуманітарний аспект. Вісник Житомир. держ. ун-ту ім. І. Франка. 2004. № 14. С 47-50.

9. Тернопільська В. І. Теоретичні засади реалізації ціннісного підходу у вихованні особистості. Нові технології навчання. 2016. Вип. 88. Ч. 2. С. 118-121.

10. Тернопільська В. І. Сучасні тенденції мотивації навчально-професійної діяльності студентів. Вісник Наџіонального університету оборони Украӥни, 2012. №5(30). C 141-144.

11. Тернопольская $\quad$ В. И., Бакулина $\quad$ О. С. Особенности формирования профессиональной компетентности будущих специалистов по налогообложению и таможенному делу. International scientific journal «Progress». Tbilisi : International Publishing House «Progress», 2018. № 1-2. P. 94-97.

12. Тернопільська В. І. Структура професійної компетентності майбутнього фахівця. Науковий вісник Мелітопольського державного педагогічного ун-ту. Серія: Педагогіка, 2012. № 9. С. 208-213. 
13. Тернопільська В. І., Чижова Н. В. Мобільність особистості як предмет наукового дослідження. Теоретико-методичні проблеми виховання дітей та учнівської молоді. 2016. Вип. 20. Кн. 2. С. 234-243.

14. Тернопільська В. І., Коломієць Т. В., Піонтківська І. О. Довідник з виховної роботи зі студентами: навч. посіб. Тернопіль : Вид-во «Богдан», 2014. 184 с.

15. Тернопільська В. І., Дерев'янко О. В. Визначення критеріїв сформованості професійної компетентності майбутніх гірничих інженерів. Наук. часопис НПУ імені М. П. Драгоманова. Пед. науки, 2012. Вип. 31. С. 264-267.

16. Babiy S., Bezkorovaina O., Matviienko O., Petko St., Ternopilska V., Soichuk R., Stanislavchuk N. Entrepreneurship model of professional development of actuaries in Canada // Journal of Entrepreneurship Education (JEI). USA. 2019. Vol: 22 Issue: 3. P. 1-6.

17. Ovsiienko Liudmyla. Innovative approaches to improving professional training of spesialists in higher educational institutions // Current scientific research: Collection of scientific articles. - Publishing house «BREEZE», Montreal, Canada, 2017. P. 215-220.

18. Ovsiienko L. M. Regularities of competently directed learning of text linguistics of studets' philological specialities // Economics, management, law: socio-economical aspects of development: Collection of scientific articles. Volum 2. Psychology. Pedagogy and Education. - Edizioni Magi - Roma, Italy. 2016. P. 245-250.

19. Pet'ko L. The development of student youth aesthetic culture on professional direction // Topical issues of contemporary science: Collection of scientific articles. - C.E.I.M., Valencia, Venezuela, 2017. P. 188-192. 


\title{
To the Question of Formation of Competences for the Safety of Life of Students with Intellectual Disabilities
}

\author{
Natalia Maksimenko \\ Assistant Professor of Special Education and Human Health \\ Central Ukrainian State Pedagogical University named after V. Vynnychenko, \\ Kirovograd, Ukraine
}

\begin{abstract}
The history of society has shown that neglect of security can lead to serious negative consequences and even tragedies, so the achievement of an adequate level of safety is possible only with the high culture and awareness of the entire population of the state. Pupils with intellectual disabilities are of particular concern because they are the most vulnerable.

The urgent issue today is to provide for the needs of each child and to create all the conditions for their full life. Children who have intellectual disabilities need special care.

Currently, the problem of socialization of children with intellectual disabilities is intensifying and their integration into society successfully. The complexity of this problem is determined by the fact that the pupils of both special schools and institutions with inclusive education have quite a variety of profound disorders of psychophysical development.

In such a case, the question arises as to the need for the formation of competencies for safety. This can be done at various levels, including the educational level, by building competencies for life safety in lessons. The formation of students' relevant competences occurs during the entire study. But a special place in this process should belong to the subject "Health Fundamentals". After all, when studying this particular subject, students have the opportunity to develop knowledge, skills and safety skills.

In his work, the author raises the issue of forming competencies for the safety of life of students with intellectual disabilities.

Keywords: competences, pupils with intellectual disabilities, guard, threat, danger.

Постановка та обгрунтування актуальності проблеми. Найбільш актуальною проблемою сьогодення є безпека життєдіяльності не лише окремої особистості, а й всього українського суспільства, що визначається дуже помітними нині тенденціями зростання кількості природних і техногенних катастроф. Основною причиною таких зрушень $є$ насамперед людський фактор, оскільки будь-яка діяльність людини у соціумі та у довкіллі здійснюється без відповідного теоретичного обгрунтування і продуманих дій. В такому випадку загострюється питання, що до необхідності формування компетентностей з безпеки життєдіяльності. Це можливо зробити на різних рівнях, зокрема на освітянському, формуючи компетентності з безпеки життєдіяльності на уроках. Формування в учнів відповідних компетентностей відбувається під час усього навчання. Але особливе місце в цьому процесі має належати навчальному предмету
\end{abstract}


«Основи здоров'я». Адже при вивчені саме цього навчального предмета учні мають можливість розвивати знання, уміння і навички з безпеки.

Аналіз актуальних досліджень і публікацій. Різним аспектам безпеки життєдіяльності людини присвячено багато теоретичних і практичних доробків таких авторів як: П. Атамчук, В. Мендерецький, О. Панчук, О. Чорна, Ю. Скобло, Н. Герман [5], О. Пуляк, А. Ткачук, Є. Желібо, О. Кобилянський, О. Кожемякін, О. Мягченко, та інші, які розглядають проблему у двох напрямках: науковотеоретичному й освітянському.

Педагогічні умови формування здоров'язбережувальної компетентності в учнів загальноосвітніх шкіл аналізують О. Аксьонова, Т. Бабко, Т. Бойченко, А. Карабашева та ін. У системі вищої школи зазначену проблему вирішують Н. Бєлікова, Д. Воронін, О. Дворнікова, О. Дорошенко, Н. Панчук та ін. Сутність поняття «педагогічні умови» досліджують О. Бойцун, Н. Бугаєць, В. Манько, Р. Серьожникова та ін.

Однак проблема формування в учнів 3 інтелектуальними порушеннями компетенції з питань безпеки життєдіяльності розглядається опосередковано. Так, дослідженню проблем життєдіяльності дітей з особливими потребами присвячені праці вітчизняних та зарубіжних науковців: Ю. Василькової, А. Колупаєвої, Т. Ілляшенко, Л. Шипіциної, Е. Даніелс та ін. Такі науковці, як Ю. Василькова, Т. Ілляшенко, А. Колупаєва зазначають, що головною умовою дитячого благополуччя є почуття захищеності батьківською любов’ю та адекватне іiі сприйняття оточуючими.

Заслуговують на увагу праці Л. Калузької, Л. Гураш, О. Лози, які визначили умови організації навчання дітей з особистої безпеки та зміст роботи з формування активної позиції щодо власного життя і здоров’я. Умови і засоби збереження власного життя, допомоги іншим у разі небезпечних ситуацій вивчали Г. Навроцька, Л. Яковенко. Проблема формування у дітей навичок здорового та безпечного способу життя є предметом досліджень Н. Авдєєвої, Н. Бібік, О. Ващенко, Г. Дудчак, С. Кондратюк, І. Калиниченко, Н. Коваль. 
Метою статті $\epsilon$ визначити основні методи формування компетентностей 3 безпеки життєдіяльності учнів початкової школи з порушеннями інтелектуального розвитку.

Виклад основного матеріалу дослідження. Формування компетенцій 3 безпеки життєдіяльності багато в чому залежить від особливостей навчальновиховного процесу закладу освіти та методів, що використовує учитель на уроці. Це створює необхідність удосконалення сучасної освіти та спонукає науковців до обгрунтування та пошуку ефективних методів формування компетентностей 3 безпеки життєдіяльності.

У навчально-виховному процесі, формування знань 3 безпеки життєдіяльності, містить усвідомлення й засвоєння світоглядних основ у сфері безпеки життєдіяльності, відповідних компетентностей та компетенції, формування принципів безпечного існування у соціумомі [10]. Все це має бути реалізовано через систему практичних занять, що спрямовані на засвоєння й усвідомлення знань, умінь і навичок з безпечної життєдіяльності.

Компетентності з безпеки життєдіяльності - це готовність самостійно вирішувати питання власного захисту і захисту оточуючих у процесі певної діяльності, вміння реагувати на небезпеки та знешкоджувати їх, користуватися принципом безпечної життєдіяльності. Суть компетентностей 3 безпеки життєдіяльності проявляється у проведенні профілактичних заходів, застосуванні технологій та методів безпечної життєдіяльності[3].

Науковець Л.Калузька зазначає, що для дитини здоров’я набуває сенсу тільки тоді, коли вона починає розуміти його значення для ефективного виконання тієї чи іншої діяльності у грі або навчанні. У створенні внутрішньої картини здоров’я бере участь вся особистість загалом. Чим складніше психіка, чим різноманітніші мотиви діяльності, тим більшого значення набуває для дитини їі стан здоров’я. У віці 6-7 років дитина не тільки здатна оцінювати своє здоров'я, але і намагається зберігати його, за постійної підтримки дорослих. Коли дитина тільки переступила поріг школи, їй важко звикати до режиму навчання, саме тому потрібна вміла, чуйна і тактовна підтримка вчителя. У своїй педагогічній діяльності педагогу важливо створювати сприятливі умови навчання дитини в школі (відсутність стресових ситуацій, 
адекватність вимог, методик виховання); забезпечувати оптимальну організацію навчального процесу, повноцінний раціонально організований руховий режим. Так, для створення сприятливого мікроклімату доцільно: перевірити освітлення, температурний режим, провірити кімнату, дотримуватись правил гігієни, чистоти в класі, створити затишок, а розсаджуючи дітей за парти, ураховувати: вади здоров'я (зір, слух), зріст, психологічну сумісність. [6]. Упроваджуючи нові прогресивні технології та інтерактивні методи навчання, учителеві слід дотримуватися правила: «Не нашкодь, навчаючи». Для цього під час навчального процесу потрібно стежити за своєчасною зміною статичної напруги під час письма та читання (фізкультхвилинки, вправи для корекції зору, музичні паузи); забезпечувати зміну різних видів навчальної діяльності, використовувати наочність з урахуваннями вимог гігієни; ураховувати позитивні емоції, які полегшують засвоєння матеріалу, що, своєю чергою, зменшує втому, стимулює вищу нервову діяльність, покращує психологічний клімат у класі. Компетенції безпеки життєдіяльності молодших школярів цілеспрямовано формуються на уроках з основ здоров'я [7]. Метою цього предмету визначено формування в учнів здоров'язбережувальних компетенцій на основі засвоєння ними знань про здоров'я та безпеку, практичних навичок здорового способу життя та безпечної поведінки, повсякденної практичної діяльності щодо збереження власного здоров'я та здоров’я інших людей, що сприятиме утвердженню ціннісного ставлення до життя і здоров'я. Науковці доводять, що компетенції $є$ особистісним утворенням, яке проявляється в процесі активних самостійних дій людини. У контексті безпеки життєдіяльності ці компетенції визначають як знання, уміння і навички, за допомогою яких людина може бути максимально готовою до всіх нестандартних ситуацій і явищ навколишнього середовища. Проведення уроків з основ здоров'я у початковій школі полягає в тому, що оволодіння комплексом компетенцій зі збереження здоров'я і безпеки життєдіяльності потребує багаторазового вправляння, насамперед у процесі групової взаємодії. Тому необхідна організація практичної, ігрової, індивідуальної та колективної діяльності учнів, що грунтується на суб'єктсуб'єктній взаємодії вчителя з учнями і учнів між собою. Такий підхід (за визначенням ООН) отримав назву «освіта на основі набуття життєвих навичок» і характеризується застосуванням інтерактивних методів 
навчання, що забезпечує ефективне засвоєння учнями навчального матеріалу i відпрацювання ними поведінкових навичок[2].

Виховання дитини з порушеннями інтелектуального розвитку вимагає врахування особливостей їх розвитку та сприймання навколишнього світу, в першу чергу - надання знань щодо їх безпеки. Тут важливого значення набуває коло спілкування (колектив однолітків як референтна група), що впливає на їх соціалізацію[1].

Ключовим моментом модернізації сучасної системи соціального супроводу та освіти є положення про створення умов для розвитку і самореалізації людини. Стратегія модернізації системи супроводу людини з особливими можливостями здоров'я та його освіти ставить перед фахівцями - спеціальними (корекційним) педагогами - нові орієнтири у визначенні корекційно-розвивальних технологій, спрямованих на підвищення можливостей до адаптації, соціалізації дітей, в тому числі і з важкими порушеннями інтелектуального розвитку. Ця стратегія безпосередньо зачіпає i спеціальні (корекційні) установи. Також необхідно відзначити, що сучасна система освіти трансформується, змінюється, визначаються нові освітні стандарти, в основу яких покладено компетентнісний підхід [8].

Використання компетентнісного підходу при навчанні учнів 3 порушеннями інтелектуального розвитку означає поступовий перехід від репродукції знань і навичок до конструювання змісту освіти, застосування різних моніторингів та використання систем контролю якості освіти. При наявності такої сильної практичної складової зміст навчального процесу буде будуватися на основі освоєння навчальних компетенцій[8]. Використання в педагогіці поняття "компетенції", як синонім "компетентності" призводить до того, що розуміння проблеми формування компетенцій стає подвійним. 3 одного боку це може означати уточнення, розширення уявлень про самих компетенціях, а з іншого - розвиток знань і умінь у учнів.

Компетентність - ця міра відповідності знань, умінь і досвіду осіб певного соціально-професійного статусу реальному рівню складності виконуваних ними завдань і розв'язуваних проблем.

Навчальна компетенція - характеристика вимог до навчального, які дозволять стати йому компетентним у навчальній діяльності. 
Дж. Равен, основоположник психологічної теорії компетентності, дає визначення "компетентності як сукупності компетенцій", підкреслюючи ї множинність. У теоретичних дослідженнях вітчизняних вчених (В.М. Галямін, І.А. Зимової, Н.А. Селезньова, В.В. Серікова, А.А. Хутірського і ін.) Відзначається, що компетентність набувається і формується в процесі життєдіяльності, і являє собою особистісну характеристику, що розглядається в двох аспектах: об'єктивному і суб'єктивному. У більшості випадків, об'єктивний аспект, який включає фундаментальні знання і вміння, реалізується в діяльності, в тому числі і трудовий. Суб'єктивний аспект характеризується позитивною мотивацією суб'єкта до даної діяльності, ціннісносмисловими уявленнями про зміст і результат. У спеціальних психолого-педагогічних дослідженнях узагальнено наукові дані, що відображають зміст і компоненти компетентності (когнітивний, мотиваційний, поведінковий, рефлексивно-оцінний), а також дано визначення базових навчальних компетенцій, таких як: навчальнопізнавальні; ціннісно-смислові; інформаційні; комунікативні; соціально-трудові. Європейський варіант ключових компетенцій включає вміння: вивчати; шукати; думати; співпрацювати; братися за справу; адаптуватися [8]. Безпека як об'єктивна реальність проявляється у відсутності або мінімальному рівні ризику виникнення збитків інтересам людини (здоров'ю, матеріальному благополуччю і т.д.) в звичайних умовах і при взаємодії з шкідливими і небезпечними чинниками життєдіяльності.

У той же час безпека життєдіяльності - це галузь наукових знань, що охоплюють теорію і практику захисту людини від небезпечних і шкідливих чинників у всіх сферах людської діяльності і середовищі проживання. [9].

Особлива небезпека підстерігає учнів початкової школи 3 порушеннями інтелектуального розвитку. Завдання дорослих полягає не тільки в тому, щоб захистити і оберігати дитину, але і в тому, щоб підготувати іiі до зустрічі з різними складними, а часом небезпечними життєвими ситуаціями.

Необхідно підкреслити такі правила поведінки, які учні зобов'язані виконувати неухильно, так як від цього залежить їхнє здоров'я і безпека. Ці правила необхідно грунтовно роз'яснювати учням, а потім спостерігати за їх виконанням. Крім цього, учні можуть опинитися в несподіваній ситуації на вулиці і вдома, тому основним завданням $є$ стимулювання розвитку в учнів самостійності і відповідальності, так як все без 
винятку, чого навчають учнів, вони повинні вміти застосовувати в реальному житті, на практиці. [5].

На нашу думку, для більш кращого засвоєння інформації про безпеку/небезпеку потрібно використовувати 3Д технології. Таким чином учні не лише побачать певну ситуації, а й ще стануть повноцінними учасниками. Так, наприклад, при моделюванні ситуації правил дорожнього руху, можна трансформувати за допомогою ЗД технологій рух машин і запропонувати учням перейти дорогу. Якщо, хтось з учнів не правильно вчинить, то шкоди здоров'ю імітована машина не принесе, а учень набуду життєвого досвіду.

Правила поведінки та заходи безпеки головним чином взаємопов'язані 3 умовами проживання людини, будь то сучасний місто або сільська місцевість, звична домашня обстановка - кожна середа диктує абсолютно різні способи поведінки і запобіжні заходи.

Висновки. Розуміючи, що учні з порушеннями інтелектуального розвитку ніколи не стануть настільки самостійними, щоб обходитися без допомоги і підтримки дорослої людини. Але кінцевою метою нашої роботи по формуванню компетенції у школярів 3 обмеженими можливостями здоров'я ми бачимо в тому, щоб навчити їх бути по можливості комунікабельними, розумно активними, які вміють звертатися по допомогу і приймати іï, бути культурними в поведінці і вести здоровий спосіб життя , бути корисними хоча $б$ в рамках своєї родини. В кінцевому підсумку це $\epsilon$ найважливішою передумовою для подальшої соціальної адаптації, яка є найважливішим завданням навчання і виховання учнів початкової школи в спеціальному (коррекционном) закладі, за допомогою можливої корекції і компенсації первинного дефекту i вторинних порушень

\section{References}

1. Atamanchuk, P.S., Menderets'kyy, V.V., Panchuk, O.P., Chorna, O.G. (2011) Bezpeka zhyttyediyal'nosti [Life Safety] navch. posib. Kyyiv. 276.

2. Tkachuk, A.I., Pulyak, O.V. (2017) Bezpeka zhyttyediyal'nosti ta osnovy okhorony pratsi. Kurs lektsiy [Safety of life and the basis of occupational safety. Course of lectures] navch. posib. Kropyvnyts'kyy. 184. 
3. Skoblo, YU.S., Sokolovs'ka, T.B., Mazorenko, D.I., Tishchenko, L.M., Troyanov, M.M. (2003) Bezpeka zhyttyediyal'nosti [Life Safety] navch. posib. Kyyiv. 424.

4. Zaporozhets', O.I., Zaplatyns'kyy, V.M., Khalmuradov, B.D., Prymenko, V.I., Mykhaylyuk, V.O., Kanitsula, T.YA. (2013) Bezpeka zhyttyediyal'nosti [Life Safety] pidruchnyk. Kyyiv. 448.

5. Herman, N.V. (2000) Bezpeka zhyttyediyal'nosti lyudyny $v$ pedahohichniy spadshchyni ukrayins 'kykh prosvityteliv (kintsya XIX - pochatku XX stolittya) [The safety of human life in the pedagogical heritage of Ukrainian educators (the end of the nineteenth and early twentieth centuries)] avtoref. dys. Kyyiv. 17.

6. Kobylyans'kyy, O.V. (2013) Kompetentnisnyy pidkhid do vyvchennya dystsyplin tsyklu bezpeky zhyttyediyal'nosti u vyshchykh navchal'nykh zakladakh [Competent approach to the study of life cycle safety disciplines in higher education institutions] Naukovyy visnyk Skhidnoyevropeys'koho natsional'noho universytetu imeni Lesi Ukrayinky. № 7. 42-47.

7. Kozhemyakin, O.S. (2015) Optymizatsiya metodolohiyi formuvannya kompetentnosti $z$ bezpeky zhyttyediyal'nosti [Optimization of the methodology of forming competence for life safety] Nauka i osvita. № 5. 45-52

8. M.V.Luzyk. Suchasni problemy formuvannya zhyznenatsional'nykh dosyahnen' ditey z obmezhenymy mozhlyvostyamy zdorov'ya zdorov'ya .// Korektsiyna ta sotsial'na pedahohika ta psykholohiya. - 2012.- №4 s.338-345

9. Polishchuk, O.V., Repins'kyy, S.V., Slabkyy, A.V. (2016) Formuvannya kompetentsiy $z$ bezpeky zhyttyediyal'nosti $v$ studentiv vyshchykh navchal'nykh zakladiv. [Formation of competences for the safety of life in students of higher educational institutions] Pedahohika bezpeky. № 1. 72-80

10. Sadovyy, M.I. (2017) Naukovo-metodychni pryntsypy eksperymental'noyi ta doslidnoyi diyal'nosti maybutnikh uchyteliv tekhnolohiy [Scientific and methodical principles of experimental and research activity of future technology teachers] Naukovi zapysky. Seriya: Problemy metodyky fizyko-matematychnoyi i tekhnolohichnoyi osvity. Vyp. 11, CH. 3. 147151. 


\title{
Translation of the Title, Name and Abstract to Author's Language
}

\author{
Анотація
}

\section{УДК 376.42}

\section{Максименко Н.В. До питання формування компетентностей 3 безпеки} життсдіяльності учнів з порушеннями інтелекту.

У статті проаналізовано стан розробленості проблеми, що досліджувалась у загальній та спеціальній психолого-педагогічній літературі; викладено результати аналізу концептуальних підходів до проблема формування компетентностей 3 питань безпеки життєдіяльності, означено сучасний стан проблеми, зокрема проблема формування компетентностей з питань безпеки життєдіяльності в учнів 3 порушеннями інтелектуального розвитку.

\section{Лimepamypa:}

1. Атаманчук П.С. Безпека життєдіяльності: навч. посіб. / Атаманчук П.С., Мендерецький В.В., Панчук О.П. Чорна О.Г. - К.: Центр учбової літератури, 2011. $276 \mathrm{c}$.

2. Ткачук А.І. Безпека життєдіяльності та основи охорони праці. Курс лекцій: навч. посіб. для студ. вищ. пед. навч. закл. всіх спец. за ОКР «бакалавр» / А.І. Ткачук, О.В. Пуляк. - [перевид. доп. та перероб.]. - Кропивницький: ПП «ЦОП «Авангард», 2017. $184 \mathrm{c}$.

3. Безпека життєдіяльності: навч. посіб. для вищ. навч. закл. III-IV рівнів акредитації / Ю.С. Скобло, Т.Б. Соколовська, Д.І. Мазоренко, Л.М. Тіщенко, М.М. Троянов. - К.: Кондор, 2003. -424 с.

4. Безпека життєдіяльності: підруч. / О.І. Запорожець, В.М. Заплатинський, Б.Д. Халмурадов, В.І. Применко, В.О. Михайлюк, Т.Я. Каніцула. - К.: Центр учбової літератури, 2013. - 448 с.

5. Герман Н.В. Безпека життєдіяльності людини в педагогічній спадщині українських просвітителів (кінця XIX - початку XX століття): автореф. дис. на здобуття наукового ступеня канд. пед. наук: спец. 13.00.01 «Загальна педагогіка та історія педагогіки» / Н.В. Герман. - К., 2000. - 17 с. 
6. Кобилянський О. Компетентнісний підхід до вивчення дисциплін циклу безпеки життедіяльності у вищих навчальних закладах / О. Кобилянський // Науковий вісник Східноєвропейського національного університету імені Лесі Українки. - 2013. - № 7. C. $42-47$.

7. Кожем'якін О.С. Оптимізація методології формування компетентності з безпеки життєдіяльності / О.С. Кожем’якін // Наука і освіта. - 2015. - № 5. - С. 45- 52. - Режим доступу: http://nbuv.gov.ua/UJRN/NiO_2015_5_10. 10. Мягченко О.П. Безпека життєдіяльності людини та суспільства: навч. пос. / Мягченко О.П. - К.: Центр учбової літератури, 2010. - 384 с.

8. М.В.Лузик. Современные проблемы формирования жизненноважных компетенцийу детей с ограниченными возможностями здоров'я.// Корекційна та соціальна педагогіка і психологія. - 2012.- №4 с.338-345

9. Поліщук О.В. Формування компетенцій з безпеки життєдіяльності в студентів вищих навчальних закладів / О.В. Поліщук, С.В. Репінський, А.В. Слабкий // Педагогіка безпеки. -2016 . - № 1. - С. 72-80.

10. Садовий М.I. Науково-методичні принципи експериментальної та дослідної діяльності майбутніх учителів технологій / M.І. Садовий // Наукові записки. Серія: Проблеми методики фізико-математичної і технологічної освіти; відп. за випуск: М.I. Садовий. / КДПУ ім. В. Винниченка. - 2017. - Вип. 11, Ч. 3. - С. 147-151. 


\title{
Development of Future Teachers` Professional Thinking by Means of Task Approach to Organization of Educational Process
}

\author{
Vyshkivska Vanda, \\ Ph.D., Associate Professor \\ Dragomanov National Pedagogical University (Ukraine Kyiv) \\ Kushniruk Svitlana \\ Ph.D., Associate Professor \\ Dragomanov National Pedagogical University (Ukraine Kyiv)
}

\begin{abstract}
The importance of the problem of developing the future teachers' professional thinking as a condition for their effective pedagogical activity is actualized in the article; different approaches of scientists to its interpretation are analyzed. Professional pedagogical thinking is interpreted as a necessary basis for providing future teachers with research skills, developing their pedagogical intuition and key operations, procedures of scientific thinking, forming professional reflection skills. The task approach to the organization of the educational process is such that it involves the introduction into the content of training programs for professionals of applied nature, which allow first to mentally construct different options for solving practical problems of different levels of complexity, to predict their effectiveness, and only then to design the plane of their application in practice, to reflect.
\end{abstract}

Keywords: professional pedagogical activity, thinking, professional pedagogical thinking, pedagogical tasks, task approach.

Сучасній історичній епосі притаманна особлива модель освіти, орієнтована на творчу ініціативу, самостійність, конкурентоспроможність майбутніх фахівців. Творчий потенціал вчителя залежить від рівня сформованості його професійного мислення, яке, в свою чергу, ініціює рефлексивну діяльність, що дає йому можливість стати професійно активним педагогом, здатним до самостійної інноваційної діяльності, i, як результат, - бути затребуваним i конкурентоспроможним.

У системі шкільної освіти відбулися серйозні зміни: диверсифікація, збагачення шкільної практики новими освітніми технологіями, формами навчання, можливістю самостійно вибирати навчально-методичну базу. Підвищення теоретичного рівня змісту навчальних дисциплін призвело до загострення протиріч між загальними цілями освіти і реальними можливостями майбутніх учителів 
вирішувати практичні завдання (нерідко має місце невміння молодих фахівців проявляти самостійність і творчо застосовувати теоретичні знання, набуті у вузі, в шкільній практиці). А сьогодні необхідний вчитель, який володіє сучасною методикою викладання предметів 3 метою формування в учнів ключових компетенцій, готовності до здійснення самостійної пізнавальної діяльності (освітньої компетенції, яка передбачає накопичення інтегрованих знань і їх застосування на практиці).

Відтак, випереджальний розвиток педагогічної освіти обумовлює необхідність розвитку у майбутнього фахівця професійного мислення, здатності оперативно реагувати на різноманітні ситуації в освітньому процесі.

Аналіз останніх досліджень. У наукових дослідженнях професійне мислення вчителя розглядається в трьох аспектах: гносеологічному (О.А. Абдулліна, А.І. Піскунов), діяльнісному (А.К. Маркова, В.А. Сластьонін), творчому (Ю.Н. Кулюткін, М.М. Кашапов та ін.). При цьому автори розглядають його як особливий склад розуму, що володіє рядом ознак, якостей і властивостей, що дозволяють говорити про педагогічне «бачення» світу (В.Е. Тамарін, Д.С. Яковлева); як «системне бачення педагогічного процесу» (Е.П. Нечитайлова); як готовність вчителя до розв'язання різноманітних педагогічних ситуацій (Л.В. Нікітенкова); як здатність застосовувати теоретичні положення педагогіки, психології та методики до конкретних педагогічним ситуацій в поєднанні з умінням «бачити» в конкретному явищі його загальну педагогічну сутність (Л.П. Маслова); як спеціальну сукупність особливостей, властивих практичному мисленню (Е.К. Осіпова).

Деякі аспекти розвитку професійного мислення через розв'язання ряду педагогічних завдань представлені в працях Л.Ф.Спіріна, М.А. Степінського, М.А. Фрумкіна та інші.

Однак формування професійного мислення в період навчання в педагогічному вузі у студентів і сьогодні відбувається не цілеспрямовано і не належним чином науково-організовано. Більш того, згідно з дослідженнями вчених (О.А. Абдулліна, Ю.Н. Кулюткін, М.М. Кашапов і ін.), професійне мислення більшості вчителів є далеко не творчим, рясніє стереотипами і методичними штампами. А тому 
необхідною є систематична спеціальна робота 3 його формування 3 використанням відповідного змісту, форм і методів.

Мета статті - на теоретичному рівні дослідити можливість вирішення педагогічної багатокомпонентної проблеми формування у майбутніх учителів професійного мислення з урахуванням основних факторів, що визначають розвиток освіти сьогодні (у період іiі модернізації), засобами задачного підходу.

\section{Виклад основного матеріалу.}

Деякі вчені (В.І. Загвязінський, Ю.Н. Кулюткін, Н.І. Мешков, Е.К. Осіпова, O.I. Чемоданова і ін.) відзначають, що творчий потенціал вчителя, впершу чергу, залежить від рівня сформованості професійного мислення і потреби у творчій діяльності. Д.В. Вількеев, В.І. Загвязінський, Н.А. Половнікова пов'язують його формування 3 вирішенням наступних конкретних завдань: 3 формуванням логічної культури мислення у студентів; з озброєнням майбутніх педагогів професійними дослідницькими вміннями i навичками; 3 навчанням переносу психологічних i педагогічних теорій, категорій, принципів і законів в конкретні педагогічні ситуації; з розвитком у студентів педагогічної інтуїції; з вихованням у майбутніх учителів і вихователів основ діалектичного мислення; 3 навчанням студентів методам та процедурам наукового мислення, з вихованням здатності до професійної рефлексії, 3 навчанням умінню вести уявний діалог інтелектуального спілкування з учнями; 3 формуванням у студентів предметного наукового мислення в сфері спеціалізації.

Ю.Н. Кулюткін, вказуючи на той факт, що саме мислення дозволяє організовувати та осмислювати інформацію, регулювати внутрішні та зовнішні аспекти діяльності педагога, до важливих якостей педагогічного мислення відносить кмітливість, діалогічність, аналітичність, технологічність, які забезпечують здатність педагога оперативно усвідомлювати та вирішувати завдання; мислити послідовно, формулювати помірковані, доказові умовисновки; детально аналізувати факти та явища, проектувати і переносити задум у площину педагогічної діяльності [9].

Г.С. Костюк, своєю чергою, наголошував, що розвиток мислення має

неабияке значення в загальному процесі формування особистості, піi розумових властивостей. Саме розвиток мислення свідчить про здатність особистості 
орієнтуватися в різних складних ситуаціях, передбачати хід подій і результати своїх власних дій, панувати над обставинами і над собою [7].

Відтак, проблема розвитку професійного мислення майбутнього вчителя пов'язана з роботами з загальної теорії мислення і грунтується на дослідженнях особливостей практичного мислення. Поняття «професійне мислення» використовується у науковій літературі в двох аспектах: коли мають на меті підкреслити високий професійно-кваліфікаційний рівень спеціаліста (якісний аспект) та коли акцентують на особливостях мислення, обумовлених характером професійної діяльності (предметний аспект).

С.Л. Рубінштейном були виділені деякі характеристики професійного мислення: витончена спостережливість, вміння використовувати для вирішення завдання особливе i одиничне в даній проблемній ситуації, уміння швидко переходити від мислення до дії і навпаки [10].

В.А. Сластьонін обгрунтовує думку про те, що специфіка педагогічного мислення визначається, 3 одного боку, особливостями мисленнєвої діяльності педагога, професійною спрямованістю пізнавальних процесів, а 3 іншого конструктивно-перетворювальними особливостями, осмисленням кожної навчальновиховної ситуації, оперативним вибором і реалізацією оптимального варіанту іiі вирішення [13].

Т. Гура визначає професійне мислення фахівця як надскладний, інтегративний, поліпроцесуальний i полісистемний феномен; особливий вид мислення, що забезпечує вирішення фахівцем проблем його професійної діяльності і професійного розвитку, функціонує на предметно- орієнтованому, діяльнісноорієнтованому та методологічному рівнях завдяки різнорівневій рефлексії [4]. Науковець також наголошує, що структура професійного мислення фахівця зумовлена особливостями його професійної діяльності та професійного становлення, має багатоплощинну i багатокомпонентну єдність процесуальних i змістових складових. Розпочинається ж воно 3 проблематизації стимульної ситуації, що знаменує умовний початок мисленнєвого пошуку [4].

Визначаючи професійно-педагогічне мислення як здатність до прогнозування, вміння поєднувати теоретичний аналіз і практичне мислення, В.В.Краєвський 
досліджує його в контексті конструктивно-діяльнісного підходу та методології культури, що актуалізує необхідність сформованості у педагога методологічної рефлексії, здатності до наукового обгрунтування, критичного мислення, творчого застосування певних концепцій, форм, методів пізнання, управлінських умінь тощо [8].

Розділяємо думку А. Орлова, який педагогічне мислення розглядає як здатність учителя аналізувати педагогічні ситуації, виокремлювати і вирішувати професійно-педагогічні завдання [10]

Розглянемо детальніше такий засіб розвитку педагогічного мислення, як навчальні задачі (завдання, ситуації). О.А. Дубасенюк визначає останні як спеціально відібрані і складені на основі типових педагогічних ситуацій, що виникають у реальній педагогічній дійсності і які вимагають від майбутніх педагогів застосування набутих психолого-педагогічних знань, оволодіння комплексом педагогічних умінь та навичок, урахування соціального замовлення суспільства, сучасних методологічних підходів, концептуальних ідей, гнучких інноваційних засобів та методів 3 метою отримання продуктивного результату у вигляді позитивних новоутворень в особистісній сфері та діяльності учня і такі, які сприяють зростанню особистості i викликають у неї потребу у саморозвитку та самовдосконаленню у майбутньому та орієнтують на досягнення успіху у подальшій професійно-педагогічній діяльності [5].

Відповідно сутність задачного підходу можна визначити як можливість «побудувати навчальне пізнання особистості як систему задач та розробити алгоритм їх розв'язання $з$ метою допомогти студентам усвідомити проблемність певних ситуацій, знайти форми та методи їх аналізу та розв'язання у процесі підготовки майбутнього фахівця у закладах вищої освіти» [6].

C.В. Сапожніков зауважує про те, що задачний підхід до навчання передбачає уведення до змісту навчальної інформації таких завдань, які активізують мисленнєві процеси студентів, закріплюють у них вміння оперувати теоретичними знаннями у практичних ситуаціях, користуватися ними при вирішенні навчальних завдань, осмислювати і бачити їх прикладний характер у професійній діяльності. Педагогічне ж завдання є одним з інструментів перетворення нових знань на елемент практики, 
найпростішою моделлю якої вони $є$. Засвоюючи певні поняття, теоретичні положення, які пропонуються студентам у вигляді мисленнєвого завдання, студенти відчувають потребу в дії [12].

У методичній підготовці майбутніх учителів у закладі вищої освіти сучасні дослідники пропонують орієнтуватися саме на професійні задачі (проектування, реалізація, аналіз) і відповідні їм функціональні одиниці діяльності. Саме тому задачі і вправи, що відповідають різним рівням рефлексії, включають визначення параметрів навчальної ситуації, аналіз дій учителя й учнів, визначення характеру й причин прояву труднощів, аналіз емоційного стану вчителя й учнів у конкретній ситуації, виділення основних і другорядних факторів, що впливають на динаміку ситуації, пошук альтернативних рішень у даній ситуації, моделювання нової навчальної ситуації, застосування методу аналізу (самоаналізу) [14].

Висновок. Таким чином, професійне педагогічне мислення - це розвинена здатність використовувати прийняті саме в даній професійній області прийоми вирішення проблемних завдань, способи аналізу проблеминх педагогічних ситуацій, прийняття професійних рішень. Задачний підхід до організації освітнього процесу у вищому педагогічному навчальному закладі забезпечує: варіативне відтворення навчального матеріалу з постановкою різноманітних цілей, застосування засобів використання знань у подібних і творчих ситуаціях, систематизацію, самостійний пошук доказів та відповідей на поставлені питання. Відтак, підготовка сучасного вчителя має бути спрямована на формування творчої ерудованої особистості фахівця, що володіє комплексом універсальних знань, професійно спрямованим мисленням, розвиненою педагогічної рефлексією, самосвідомістю, нарешті, громадянина, який чітко усвідомлює відповідальність за свої дії, наслідки яких проектуються не тільки в сьогодення, а й у майбутнє.

Перспективи подальших досліджень пов’язані 3 визначенням особливостей розвитку професійного мислення у майбутніх учителів гуманітарного профілю.

\section{References}

1. Vyshkivska V.B. Rozvytok profesiinoi kompetentnosti maibutnikh fakhivtsiv shliakhom pedahohichnoho proektuvannia kulturno-osvitnoho seredovyshcha zakladiv vyshchoi osvity [Development of professional competence of future specialists through 
pedagogical designing of cultural and educational environment of higher education institutions] // Estetyka i etyka pedahohichnoi dii: zb.nauk.prats / In-t ped.osvity i osvity doroslykh NAPN Ukrainy.- Poltava, 2018. - S. 172-181.

2. Vyshkivska V.B., Kushniruk S.A. Do problemy formuvannia profesiinoi kompetentnosti vchytelia Novoi ukrainskoi shkoly. [To the problem of formation of professional competence of the teacher of the New Ukrainian school] Naukovyi chasopys Natsionalnoho pedahohichnoho universytetu imeni M.P. Drahomanova. Seriia №5. Pedahohichni nauky: realii ta perspektyvy. Vypusk 64. Kyiv: Vyd-vo NPU imeni M.P. Drahomanova. 2018. S. 44-48.

3. Vyshkivska V.B. Modeliuvannia osvitnoho seredovyshcha yak umova vdoskonalennia profesiinoi pidhotovky fakhivtsiv [Modeling the educational environment as a condition for improving the professional training of specialists] // Materialy naukovoi konferentsii vykladachiv kafedry teorii ta istoriipedahohiky / Za nauk. red. L.P. Vovk, V.B. Vyshkivskoi. K.: Vyd-vo NPU imeni M.P.Drahomanova, 2018. S. 95-103.

4. Hura T. Profesiine myslennia: mekhanizm uspishnoi/nevdaloi sotsializatsii fakhivtsia [Professional Thinking: The Mechanism of Successful / Unsuccessful Socialization of the Specialist] //Visnyk Kyivskoho Natsionalnoho universytetu imeni Tarasa Shevchenka. №1 (3). 2015. S. 26-29

5. Dubaseniuk O.A. Realizatsiia zadachnoho pidkhodu u profesiinii pidhotovtsi maibutnoho vchytelia [Implementation of the task approach in the future teacher's professional training] // Novi tekhnolohii navchannia: Materialy mizhnarodnoi konferentsii "Dukhovno-moralne vykhovannia i profesionalizatsiia: vyklyky KhKhI st. ] // № 66. Ch. 1: Instytut innovatsiinykh tekhnolohii i zmistu osvity Ministerstva osvity i nauky Ukrainy, Akademiia mizhnarodnoho spivrobitnytstva z kreaktyvnoi pedahohiky. Kyiv-Vinnytsia, 2010. S. 159-164

6. Zhelanova V.V. Kontekstne navchannia maibutnoho vykladacha pochatkovykh klasiv: teoriia i tekhnolohiia: monohrafiia. [Contextual education of the future elementary school teacher: theory and technology: monograph] Luhansk: LNU imeni Tarasa Shevchenka, 2013. 484 s.

7. Kostiuk H.S. Psykholohiia. Pidruchnyk dlia pedahohichnykh vuziv [Psychology. Textbook for teachers] / za redaktsiieiu prof. H.S. Kostiuka. K.: Radianska shkola. 1961. S. 257.

8. Kraevskyi V.V. Kontseptualnost kursa pedahohyky kak uslovyia formyrovanyia u budushchykh uchytelei konstruktyvno- deiatelnostnoi pozytsyy [Conceptualisation of the course of pedagogy as a condition of formation of constructive-activity position in future teachers] // Pedahohyka kak nauka y uchebnыi predmet. Tula: Yzd-vo THPU, 2000. Ch.1. 217 s. S.16.

9. Kuliutkyn Yu.N. Yndyvydualnble razlychyia v mblslytelnoi deiatelnosty vzroslblkh uchashchykhsia. [Individual differences in the thinking activity of adult learners] M.: Pedahohyka, 1971. $111 \mathrm{~s}$.

10. Orlov A. A. Professyonalnoe myshlenye uchytelia kak tsennost [Teacher's professional thinking as a value ] //Pedahohyka. 1995. № 6. S. 63-68. 
11. Rubynshtein S.L. O myshlenyy y putiakh eho yssledovanyia. [About thinking and ways of its research.] M.: Nauka, 1958. 220 s.

12. Sapozhnykov S.V. Naukovo-praktychni pidkhody do orhanizatsii navchalnoho protsesu $v$ pedahohichnykh universytetakh $i$ koledzhakh krain chornomorskoho rehionu [Scientific and practical approaches to the organization of educational process in the pedagogical universities and colleges of the Black Sea region] // Pedahohichni nauky. 2014. № 2 (8). S. 115-121.

13. Slastenyn V.A. Metodolohycheskaia kultura uchytelia [Methodological teacher culture] /V.A. Slastenyn, V.E. Tamaryn // Sovetskaia pedahohyka. 1990. №7. S. 82-88.

14. Solovova E.N. Metodycheskaia podhotovka y perepodhotovka uchytelia ynostrannoho yazyka: yntehratyvno-refleksyvnbil podkhod: monohrafyia. [Methodical preparation and retraining of a foreign language teacher: an integrative-reflexive approach: a monograph] M.: HLOSSA-PRESS, 2004. 336 s.

15. Kushniruk S.A. Formation of readiness for research activity of students in the process of general-pedagogical preparation//Science and practice: Collection of scientific articles. Thorpe Bowker. Melbourne, Australia, 2016. P. 262-267.

16. Kushniruk S.A. Normative and legal frameworks of students' scientific and research activity in pedagogical universities of Ukraine(the $20^{\text {th }}-$ beginning of the $21 \mathrm{st}$ century) // Perspective directions of scientific researches: Collection of scientific articles. Agenda Publishing House, Coventry, United Kingdom, 2016. P. 302-305.

\section{Translation of the Title, Abstract and References to the Author's Language}

\section{УДК 378.091.3:159.955:37091.2}

\section{Вишківська В.Б., Кушнірук С.А. Розвиток професійного мислення майбутніх учителів засобами задачного підходу до організації освітнього процесу.}

У статті актуалізується значущість проблеми розвитку професійного мислення майбутніх учителів як концептуально важливої умови здійснення ними результативної педагогічної діяльності; аналізуються різні підходи науковців до його тлумачення. Професійне педагогічне мислення трактується як необхідна основа озброєнням майбутніх педагогів дослідницькими вміннями, розвитку у них педагогічної інтуїції та ключових операцій i процедур наукового мислення, формуванням навичок професійної рефлексії. Задачний підхід до організації освітнього процесу є таким, що передбачає введення до змісту програм підготовки фахівців педагогічних завдань прикладного характеру різного рівня складності, які дозволяють спочатку мисленнєво конструювати різні варіанти їх розв'язання, прогнозувати результативність, i тільки після цього проектувати площину застосування у практичній діяльності, здійснювати рефлексію

Ключові слова: професійна педагогічна діяльність, мислення, професійне педагогічне мислення, педагогічні задачі, задачний підхід 


\section{Література}

1. Вишківська В.Б. Розвиток професійної компетентності майбутніх фахівців шляхом педагогічного проектування культурно-освітнього середовища закладів вищої освіти// Естетика і етика педагогічної дії: зб.наук.праць / Ін-т пед.освіти і освіти дорослих НАПН України. Полтава, 2018. С. 172-181

2. Вишківська В.Б., Кушнірук С.А. До проблеми формування професійної компетентності вчителя Нової української школи. Науковий часопис Наиіонального педагогічного університету імені М.П.Драгоманова. Серія №5. Педагогічні науки: реалії та перспективи. Випуск 64. Київ: Вид-во НПУ імені М.П. Драгоманова. 2018. C. 44-48.

3. Вишківська В.Б. Моделювання освітнього середовища як умова вдосконалення професійної підготовки фахівців // Матеріали наукової конференції викладачів кафедри теорії та історіїпедагогіки / За наук. ред. Л.П. Вовк, В.Б. Вишківської. К.: Вид-во НПУ імені М.П.Драгоманова, 2018. С. 95-103.

4. Гура Т. Професійне мислення: механізм успішної/невдалої соціалізації фахівця //Вісник Київського Національного університету імені Тараса Шевченка. №1 (3). 2015. C 26-29

5. Дубасенюк О.А. Реалізація задачного підходу у професійній підготовці майбутнього вчителя // Нові технології навчання: Матеріали міжнародної конференції "Духовно-моральне виховання і професіоналізація: виклики XXI ст.] // № 66. Ч. 1: Інститут інноваційних технологій і змісту освіти Міністерства освіти i науки України, Академія міжнародного співробітництва з креактивної педагогіки. Київ-Вінниця, 2010. С. 159-164.

6. Желанова В.В. Контекстне навчання майбутнього викладача початкових класів: теорія і технологія: монографія. Луганськ: ЛНУ імені Тараса Шевченка, 2013. 484 с.

7. Костюк Г.С. Психологія. Підручник для педагогічних вузів / за редакцією проф. Г.С. Костюка. К.: Радянська школа. 1961. С. 257.

8. Краевский В.В. Концептуальность курса педагогики как условия формирования у будущих учителей конструктивно- деятельностной позиции / В. В. Краевский // Педагогика как наука и учебный предмет. Тула: Изд-во ТГПУ, 2000. Ч.1. 217 с. С.16.

9. Кулюткин Ю.Н. Индивидуальные различия в мыслительной деятельности взрослых учащихся / Ю.Н. Кулюткин, Г.С. Сухобская. М.: Педагогика, 1971. $111 \mathrm{c}$.

10. Орлов А.А. Профессиональное мышление учителя как ценность// Педагогика. 1995. № 6. С. 63-68.

11. Рубинштейн С.Л. О мышлении и путях его исследования. М.: Наука, 1958. $220 \mathrm{c}$.

12. Сапожников С.В. Науково-практичні підходи до організації навчального процесу в педагогічних університетах і коледжах країн чорноморського 
регіону / Вісник Дніпропетровського університету імені Альфреда Нобеля Серія «Педагогіка і психологія» // Педагогічні науки. 2014. № 2 (8). С. 115-121

13. Сластенин В.А. Методологическая культура учителя / В.А. Сластенин, В.Э. Тамарин // Советская педагогика. 1990. №7. С. 82-88.

14. Соловова Е.Н. Методическая подготовка и переподготовка учителя иностранного языка: интегративно-рефлексивный подход: монография. $\mathrm{M}$.: ГЛОССА-ПРЕСС, 2004. $336 \mathrm{c}$.

15. Kushniruk S.A. Formation of readiness for research activity of students in the process of general-pedagogical preparation //Science and practice: Collection of scientific articles. Thorpe Bowker. Melbourne, Australia, 2016. P. 262-267.

16. Kushniruk S.A. Normative and legal frameworks of students' scientific and research activity in pedagogical universities of Ukraine(the $20^{\text {th }}$ - beginning of the $21 \mathrm{st}$ century) // Perspective directions of scientific researches: Collection of scientific articles. Agenda Publishing House, Coventry, United Kingdom, 2016. P. 302-305. 


\title{
Consideration of the Future Music Teacher's Performing Skill in the Aspect of Forming His / Her Performing Mobility
}

\author{
Kalishuk Oleksandra \\ ORCID: http://orcid.org/0000-0002-0120-7834 \\ Concertmaster at the Department of Theory and Methods of Voice Production \\ Faculty of Arts named after A. Avdiievskyi \\ Dragomanov National Pedagogical University (Ukraine, Kyiv,)
}

\begin{abstract}
Considered the raising level of performing skills in future Music teacher in the way of forming his performing mobility. The specificity of the instrumental training students is presented and described the various scientific views their professional competence as a teacher and a musician.

Based on teacher observation and analysis of performance practice, the impact skills of improvisation on performing activity of a musician is defined. Disclosed the essence and meaning notion of «improvisation skills» of future Music teacher.

The essence of the peculiarities of the pianist's performance is revealed; structural analysis of its components (information-need, operational-technological, emotional-evaluative and social-communicative) is described. Defined the concepts performing competence and performing activity. Analyzed the abilities of a music teacher to ensure his ability to perform.
\end{abstract}

Keywords: future Music teacher, performing competence, performing mobility, the components of performing mobility.

Актуальність. Динамізм та швидкоплинність сучасних соціальних процесів змінюють умови багатопрофільної та поліфункціональної виконавської діяльності учителя музики, що полягає у синтезі музичного мистецтва та педагогічного впливу на учнів. Це зумовлює необхідність розвитку його здатності до швидкої адаптації щодо змінних умов професійної діяльності та оперативного реагування на ці зміни, а отже, формування його виконавської мобільності $[4 ; 5 ; 6 ; 12 ; 13 ; 14 ; 15]$.

У нашому сьогоденні, щоб бути конкурентноздатним до вимог, які окреслюються для педагога-музиканта закладу загальної середньої освіти (ЗЗСО), треба володіти не тільки високим рівнем професійної компетентності та педагогічної майстерності, але й бути спроможнім до самореалізації, самовдосконалення у своїй професійній діяльності. Через що, специфіка підготовки висококваліфікованого вчителя музики полягає у вихованні одночасно виконавця-музиканта і педагога. За один урок учитель музичного мистецтва в закладі загальної освіти повинен навчати своїх учнів музичної грамоти, сприяти оволодінню учнями навичками слухання музики, розвивати в достатній мірі музичні здібності, співацький голос, ритмічне чуття, надати відомості 
про музичні твори та їх авторів. Педагог-музикант доносить до свідомості слухачівучнів ідеї та художньо-образний зміст музичного твору й авторських засобів музичної виразності. При цьому вчитель музики здійснює словесні пояснення або ставить певні завдання своїм учням. Така діяльність відрізняється від професійної діяльності виконавця-артиста, яка виражається лише в інтерпретації музичного твору без його словесного пояснення.

Виклад основного матеріалу. Українська вчена Г. Падалка називає музичну культуру і педагогічну майстерність основними факторами професійної підготовки майбутніх вчителів музики. Важливо, щоб вчитель музики міг виразно на високому професійному рівні виконувати музичний твір; особливе значення має уміння вчителя образно, цікаво, доступно розповісти учням про музику, дати словесну інтерпретацію музичної образності таким чином, щоб спрямувати сприйняття учнів та пробудити в них любов до музики [8, с. 62].

Професійна підготовка вчителя музики повинна забезпечити можливість його адаптивності до змін соціокультурного середовища. Рівень такої адаптивності залежить від того, як швидко він знайде взаєморозуміння з колегами та учнями, керівниками колективів, а також буде здатним до варіативної зміни діяльності у відповідності до поставлених педагогічних задач. Початком процесу такої адаптації в соціумі буде усвідомлення вчителем того факту, що стереотипи, які були засвоєні в попередній діяльності, не забезпечують досягнення успіху і стають причиною необхідності перебудови поведінки у відповідності до вимог нових соціальних ситуацій. I, коли в процесі діяльності вчителя музики коригуються власні установки, ламаються шаблони та стереотипи, це свідчить про наявність у нього професійної мобільності.

На думку Л. Арчажнікової основою професії учителя музики $є$ синтез педагогіки та музичного мистецтва, які формують його музично-педагогічну діяльність. У процесі вивчення спеціальних дисциплін майбутній вчитель музики набуває таких педагогічних знань та умінь: уміння знайти репертуар та будувати музичний літературний матеріал; володіння виразним мовленням для впливу на учня та пробудження його емоційної сфери; встановлення доцільної манери спілкування 3 учнями; контролювання та здатність об’єктивно оцінювати свої педагогічні дії [1, с. 243]. Такий підхід свідчить про єдність педагогічного та виконавського аспектів 
підготовки майбутнього вчителя музики.

Виконавська компетентність майбутнього учителя музики виступає як сукупність, взаємодія особистісного, когнітивно-діяльнісного та рефлексивного компонентів, ступінь сформованості яких дозволяє йому ефективно здійснювати музично-виконавську діяльність.

Зупинимося на розгляді визначених нами вище компонентів.

Особистісний компонент охоплює музичні здібності, емоційно- вольові якості майбутнього вчителя музики і $є$ провідним компонентом його виконавської компетенції . Особистісний компонент відображає психологічну готовність та здібність майбутнього вчителя до здійснення виконавської діяльності та удосконалення власного досвіду.

Когнітивно-діяльнісний компонент виконує інформаційну, орієнтаційну та трансляційну функції, представляє собою єдність педагогічних, культурологічних, музично- теоретичних та спеціальних знань, а також комплекс умінь, які забезпечують практичну реалізацію виконавської компетентності майбутнього учителя музики.

Рефлексивний компонент містить в собі як критичні, так і евристичні аспекти, виступає як джерело нового знання , виконує регуляторну функцію. Він характеризує здатність до осмислення, самоаналізу та самооцінки майбутнім учителем музики власної музично-виконавської діяльності та її результатів , спроможність до уточнення на підставі власного досвіду оптимальних методів та прийомів роботи над музичним твором [7, с. 45].

Отже, виконавська діяльність являє собою постійний творчий пошук, заснований на діалектичній єдності об'єктивного першоджерела, яке закладено в точному авторському тексті, та суб'єктивного ставлення, пов'язаного 3 творчою ініціативою самого виконавця. Наочним зразком може слугувати опис у наукових доробках Л. Петько створення музичних образів головних персонажів вірша М. Ховітт «The Spider and the Fly» американським композитором і піаністом Вільямом Гіллоком [13, с. 398], акцентуючи увагу на тому, що робота над музичним твором починається 3 розуміння художнього образу і звукового ідеалу твору в цілому та суттєвих у нашому предметі розгляду - образів Павука і Мухи [6, с. 186-187], коли українська піаністка Вікторія Єрмольєва шляхом засвоєння художнього тексту вірша «Паук і Муха», розуміння його головних образів демонструє у своїй творчості «феномен 
метадіалогічності» (багаторівневу міжсуб'єктну взаємодію, що має складну ієрархічну детермінацію і породжує неперервну інтерпретацію текстових структур у різних знакових системах, як особливе свойство музичної свідомості особистості [6, с. 186].

Музичне виконавство, на думку Н. Яранцевої, є одним з найбільш дієвих видів творчої діяльності, так як воно являє собою безпосереднє динамічне спілкування музиканта та слухача. Виконавська діяльність учителя музики є багатопрофільною та поліфункціональною, тому що поєднує багато різноманітних функцій та ролей, які характеризують його функціональність як інструменталіста, вокаліста та хормейстера. Головною якістю такої діяльності є виконавські можливості музиканта, що залежать: від вроджених психологічних, фізіологічних та морфологічних особливостей людини; від набутих знань та умінь, які дозволяють йому ефективно використовувати ці особливості [11, c. 96].

Наголосимо, що виконавська діяльність вчителя музики базується як на основі специфічних технічних навичок, так і на музично-теоретичних знаннях, на вмінні осягнути сенс музичної мови та відтворити його у конкретному звучанні. До особливих якостей вчителя музики в аспекті його виконавської діяльності належить вміння виконувати інструментальні твори різного стилю; вміння розкривати художній образ музичного твору шляхом точного прочитання нотного тексту та власного виконавського досвіду; володіння навичками самостійної роботи над музичним твором; опанування додаткового інструментального репертуару засобом «читання 3 аркушу», знання специфіки виконання репертуару шкільної програми із слухання музики, яке виражається у вмінні виконувати фрагменти творів, акцентувати увагу дітей на найбільш виразних чи значимих моментах; формування та розвиток концертмейстерських навичок: виконання акомпанементу до вокально-хорових та камерно-інструментальних творів; розвиток творчих навичок, що характеризуються імпровізацією, грою «на слух», транспонуванням, створенням нескладних супроводів до пісенного репертуару, аранжування творів та ін. [2, с. 55-56].

Тому, сказане вище дозволяє стверджувати, що виконавська діяльність вчителя музики $є$ інтегрованою властивістю його особистості і характеризується технічними уміннями, здатністю до творчих пошуків у процесі інтерпретації музичних образів. Рівень оволодіння цією діяльністю визначає ступінь підготовленості вчителя музики до 
його професійної роботи в школі.

Для успішної професійної діяльності майбутнього учителя музики йому необхідно не тільки мати професійні знання та уміння. Учитель у процесі своєї виконавської діяльності повинен бути здатним до швидкої адаптації, уміти оперативно реагувати на ті зміни, що відбуваються під час уроку. Розглянемо вказані здібності як якісну характеристику учителя, що забезпечує здатність його швидкого реагування на зміни у сфері виконавської діяльності, тобто виконавську мобільність.

На думку Є. Федоровича, здатність до переключення уваги, іiї «гнучкість» формує виконавську мобільність [9, с. 132]. Переключення уваги з одного об'єкта на інший означає свідоме та осмислене її переміщення. В такому випадку очевидно, що переключення уваги у будь-якій складній ситуації, що швидко змінюється, означає здатність швидко орієнтуватись цій ситуації та визначати або враховувати значимість змін включених в неї елементів. Вміння миттєво переключати увагу дозволяє порівняти свої дії з діями учнів, досягати метроритмічної, артикуляційної, динамічної злагодженості в ансамблі.

Аналіз літературних джерел показав, що дослідженню виконавської мобільності учителя музики у єдності ії складових, що характеризують суть дане поняття, не приділялося достатньої уваги, не було комплексного підходу до розгляду цього питання.

О. Баланко під виконавською мобільністю вважає відкритість музичновиражальної системи виконавця, універсальність професійного потенціалу, здатність кардинально змінювати виконавський арсенал, інтелектуальну рухомість, легкість і швидкість у прийнятті «теоретичних» рішень щодо виконавської техніки як засобу втілення художнього образу музичного твору [2, с. 78]. Особливість виконавської мобільності характеризується сполучуваністю, взаємодією, взаємопроникненням та взаємозамінним характером складових частин, які забезпечують іiі якість, а саме: 1) мотиваційна мобільність, що представляє собою динамічну готовність до реалізації власних можливостей у різних функціональних видах діяльності (інструменталіствиконавець, концертмейстер); 2) інтелектуальна мобільність - це спосіб орієнтуватися в творчому музичному просторі, гнучкість мислення, які дозволяють знаходити нестандартні рішення, творча ініціатива; 3) емоційно-вольова мобільність представляє собою динамічне вміння здійснювати адекватну самооцінку, самоконтроль, 
самодіагностику, аналіз результатів власної виконавської діяльності, керування своїм психічним станом; 4) ригідна мобільність характеризує легкість пристосування особистості до ситуації, що змінюється, легкість зміни установок та суджень; 5) інтерпретаційна мобільність розглядається як здатність до динамічної, спонтанної інтерпретації музичного твору, що відрізняється від точного слідування тексту, який був написаний раніше; 6) особистісна мобільність представляє собою цілеспрямовану активність особистості із саморозвитку та самовдосконалення.

Тому, виконавська мобільність формує здатність до варіативності зміни ходу та змісту виконавської діяльності, дозволяє гнучко долати ії труднощі та «штампи», легко та швидко приймати рішення в швидкоплинних творчих ситуаціях, здійснювати вибір найбільш вдалого з професійної точки зору рішення поставлених творчих завдань.

У контексті вивчення феномену виконавської мобільності педагога-музиканта визначимо їі структурні складові, в основу яких покладено принцип цілісного поєднання компонентів. Додамо, що у філософській літературі структуру тлумачать як засіб закономірного зв’язку між складовими предметів і явищ природи і суспільства, мислення і пізнання; як сукупність істотних зав’язків виділених частин цілого, що забезпечує його єдність [3, с. 978].

Виокремимо ключові (опорні) слова, які охоплюють поняття «мобільність» як систему. Отже розглянемо їх послідовно: 1) інформаційно-потребнісний компонент: передбачає потребу у досконалому оволодінні виконавською діяльністю, реалізується в цілому у прагненні майбутнього педагога-музиканта до самовдосконалення у професійній діяльності, а також до реалізації власного виконавського творчого потенціалу в умовах конкурсних змагань і публічних виступів; 2) операційнотехнологічний компонент: інструментально-виконавські уміння та навички, з однієї сторони, та сукупність якостей особистості, які дозволяють успішно здійснювати професійну діяльність, 3 іншої, формують інтегрований показник професійної майстерності музиканта. Рівень інструментально-виконавської майстерності залежить від технічних засобів (умінь і навичок) музиканта для гри на інструменті, емоційновольових якостей та індивідуально-типологічних властивостей (характер, здібності, темперамент) конкретного музиканта. 3) Емоційно-оцінний компонент: осягнення емоційно-образного змісту твору здійснюється завдяки реалізації двох задач, де перша 
3 них - інтелектуальна - полягає у тому, щоб зрозуміти та усвідомити задум композитора. Реалізація цієї задачі повинна трансформуватись у сферу наступної емоційної задачі, яка характеризується емоційними переживаннями авторського задуму, що реалізує музикант-інструменталіст, виражаючи таким чином своє емоційне ставлення до музичного твору. Можливість музиканта виражати у музичному творі багатогранність емоційних хвилювань залежить від його внутрішніх здатностей генерувати у своїй психіці переживання.

4) Творчо-комунікативний компонент: володіння навичками соціокультурної комунікації, що дозволяє музиканту-інструменталісту швидше та 3 мінімальними психофізичними витратами включитись у професійний процес взаємодії з партнерамимузикантами зі спільної виконавської діяльності та різними категоріями слухачів, досягати злагодженості виконавських дій, створювати позитивний емоційний клімат у творчому колективі, що в кінцевому результаті, повинно додати динамізму та ефективності виконавській діяльності. Наполегливість, ініціативність, самостійність, витримка та саморегуляція, сміливість та рішучість - всі ці риси поведінки по-різному проявляються в діяльності музиканта.

Викладене вище, ще раз засвідчує, що специфіка професійної підготовки майбутнього вчителя музики визначається необхідністю інтегрованої взаємодії елементів, що входять в поняття «вчитель» та поняття «музикант». Багатопланова діяльність вчителя музики передбачає синтез вказаних понять, які формують професійні засади його підготовки, на базі психолого-педагогічних та фахових знань та умінь.

Висновки. Отже, проведений структурно-функціональний аналіз виконавської мобільності майбутнього вчителя музики уможливив пояснення сутності та специфіки iii компонентів, а також доводить, що в практичній діяльності педагога-музиканта усі вищезазначені компоненти синтезовані, створюють єдину систему, спрямовану на цілісне формування досліджуваного феномену у майбутніх вчителів музики вищих на художньо-педагогічних закладів освіти.

Розглянуті шляхи формування виконавської мобільності майбутнього педагогамузиканта, екстраполюючись у площину фортепіанної підготовки майбутніх учителів музики, дали змогу виокремити специфічні особливості означеної підготовки, які, в першу чергу, полягають у іï педагогічній спрямованості. Педагогічно спрямована 
фортепіанна підготовка формує знання, уміння та емоційні компетенції, необхідні для умов педагогічної діяльності, що стрімко змінюються. Це уможливлює формування здатності майбутнього педагога-музиканта швидше входити до професійного музичного середовища, набуваючи професійний досвід та оволодіваючи його стандартами та цінностями.

Нами описано складові виконавської мобільності, які характеризують різноманітність функцій та умінь майбутнього учителя музики, а проведений аналіз дозволив сформулювати поняття «виконавська мобільність» майбутнього учителя музики як інтегративний, динамічний показник, щуо характеризує здатність до варіативних змін в процесі виконавської діяльності з метою найбільш вдалого вирішення ситуаційних виконавських проблем.

Подальше вивчення виконавської мобільності майбутнього учителя музики передбачає змістовно-структурний аналіз даного феномену.

\section{References}

1. Archazhnikova L. G. Teoreticheskie osnovy professionalno-pedagogicheskoj podgotovki uchitelya muzyki [Theoretikal foundations of professionally-pedagogic treining of teachers of music]: diss. doktora ped.nauk: 13.00.0. Moscow,1986. 505 p.

2. Blanko O. M. Vikonavska mobilnist yak chinnik rozvitku suchasnoyi kamernoyi vokaknoyi muziki [Performing mobility as a factor in the development of modern chamber vokal music]. Mistetstvoznavski zapiski: zb. nauk. statey. Kyiv: Milenium, 2016. Issue 29. P.74-83.

3. Istoriya filosofii. Entsiklopeliya [History of philosophy. Encyclopedia] /compiled by Gritsanov A.A. Minsk: Knizhnyiy dom, 2002. 1376 p.

4. Padalka Gh. M.Pedaghoghika mystectva (Teorija $i$ metodyka vykladannja mystecjkykh dyscyplin) [Art pedagogics (Theory and methodology of teaching art courses)]. Kyiv: Osvita Ukrajiny, 2008. 274 p.

5. Pet'ko L.V. Robota nad pisneju v kursi anglijs'koi' movy jak odyn iz zasobiv profesijnoi' pidgotovky studentiv gumanitarnyh special'nostej VNZ [The song in English course as a means of training humanities majors university students']. Inozemni movy. 2011. No. 1. P. 44-48.

6. Pet'ko L.V. Formuvannya profesiyno orientovanogo inshomovnogo navchalnogo seredovishcha v umovah universitetu dlya studentiv spetsialnosti «Muzichne mistetstvo» (na prikladi virsha Meri Hovitt «Pavuk $i$ Muha») [Formation of professionally oriented foreign language teaching environment in the terms of university for students of Music Art specialty (on illustration of a poem «The Spider and the Fly» by Mary Howitt)] Naukovi zapiski Berdyanskogo derzhavnogo pedagogichnogo universitetu. Pedagogichni nauki: zb. nauk. pr. Issue 1. Berdyansk : FO-P Tkachuk O.V., 2016. P. 184-190. 
7. Sizova E. Z. Kommunikativnaya kultura kak faktor povysheniya kachestva professionalnoj podgotovki studentov muzykalnogo kolledzha [Communicative culture as a factor of improving the quality in vocational training of students at a musical college. Srednee professionalnoe obrazvanie. 2007. No. 12. P. 44-46.

8. Teoriya i metodika mistetskoyi osviti.Naukova shkola G.M.Padalki [ Theory and methodology of art education. Scientific school G.M.Padalki] : kolektivna monografiya /nauk.red. A.V. Kozir. Kyiv : NPU im.Dragomanova, 2010. 360 p.

9. Fedorovich E. N. Osnovy muzykalnoj psikhologii [Basics of music psychology] : ucheb. posobie. Ekaterinburg, 2007. 206 p.

10. Shcholokova O. P. Osnovni tendentsiyi fortepiannoyi diyal'nosti pedahohichnomuzychnoyi tvorchosti u inshykh muzychnykh navchal'nykh zakladakh [Major trends of piano activity of pedagogical and musical creativity in other musical educational establishments] // Topical issues of education: collective monograph. Lisbon, Portugal : Pegasus Publishing, 2018. P. 205--219.

11. Yaranceva N.YA. Khudozhestvennaya deyatelnost. Problemy subekta i obekta determinatsii [Art activity. Problems of subject and object of determination]. Kyiv: Naukova dumka. $244 \mathrm{p}$.

12. Pet'ko L. V. Formation of professionally oriented foreign language teaching environment in the conditions of university for students of specialties 023 «Fine Arts» and 022 «Design» // Economics, management, law:realities and perspectives: Collection of scientific articles. Psychology. Pedagogy and Education. - Les Editions L'Originale, Paris, France. 2016. P. 466-470.

13. Pet'ko L. V. Formation of professionally oriented foreign language teaching environment in the conditions of university for students of art specialties // Economics, management, law: problems of establishing and transformation: Collection of scientific articles. - Al-Ghurair Printing \& Publishing LLC, Dubai, UAE, 2016. P. 395-398.

14. Shcholokova O. P. Art and pedagogical designing as a means of improvement of music teacher's professional preparing // Economics, management, law: socio-economical aspects of development: Collection of scientific articles. Volum 2. - Edizioni Magi - Roma, Italy. 2016. P. 265-268.

15. Shcholokova Olga. Modern Views on the problem Piano tone production in the musicalpedagogical literature / Olga Shcholokova, Wang Xue // Intellectual Archive. Toronto : Shiny Word.Corp. (Canada). 2016. November/December. Vol. 5. No. 6. PP. 81-90.

Translation of the Title, Abstract and References to the Author's Language

УДК378.011.3-/05/:78\}:7.071.3

Калішук О. Д. Розгляд виконавської майстерності майбутнього вчителя музики в аспекті формування його виконавської мобільності.

Розглянуто актуальні питання підвищення рівня виконавської майстерності майбутнього учителя музики в аспекті формування його виконавської мобільності. Представлено специфіку інструментальної підготовки студентів даного профілю та проаналізовано різні наукові погляди щодо їх професійної компетентності як педагога 
та музиканта. Розкрито сутність особливості виконавської мобільності студентівпіаністів; описано структурний аналіз іiі компонентів: інформаційно-потребнісного, операційно-технологічного, емоційно-оцінного та соціально-комунікативного. Визначено поняття виконавської компетентності та виконавської діяльності. Досліджені здібності учителя музики, що забезпечують його здатність до виконавської мобільності.

Ключові слова: майбутній вчитель музики, виконавська мобільність, компоненти виконавської мобільності.

\section{Література}

1. Арчажникова Л. Г. Теоретические основы профессионально-педагогической подготовки учителя музыки: дисс. ... д-ра пед. наук: 13.00.01. Москва 1986. 505 с.

2. Бланко О. М. Виконавська майстерність як чинник розвитку сучасної камерної вокальної музики. Мистецтвознавчі записки. Київ: Milenium, 2016. Вип. 29. Р. 74-83.

3. Історія філософії : Енциклопедія . Москва : Книжковий дім, 2002.

4. Падалка Г.М. Педагогіка мистецтва (Теорія і методика викладання мистецьких дисциплін) : [монографія]. Київ: Освіта України, 2008. 274 с.

5. Петько Л. В. Робота над піснею в курсі англійської мови як один із засобів професійної підготовки студентів гуманітарних спеціальностей ВНЗ. Іноземні мови. 2011. № 1. С. 44-48.

6. Петько Л. В. Формування професійно орієнтованого іншомовного навчального середовища в умовах університету для студентів спеціальності «Музичне мистецтво» (на прикладі вірша Мері Ховітт «Павук і Муха»). Наукові записки Бердянського державного педагогічного університету. Педагогічні науки: зб. наук. пр. Вип. 1. Бердянськ : ФО-П Ткачук О.В., 2016. 184-190.

7. Сизова Е. 3. Коммуникативная культура как фактор повышения качества профессиональной подготовки студентов музыкального колледжа. Среднее профессиональное образование. 2007. № 12. С. 44-46.

8 Теорія і методика мистецької освіти. Наукова школа Г.М.Падалки : колективна монографія / наук. ред. А. В. Козир. Київ : Вид-во НПУ імені Н. П. Драгоманова , 2010. $360 \mathrm{c}$.

9. Федорович Е. Н., Тихонова Е. В. Основи музикальной психологии : учеб. пособие. Екатеринбург, 2007. 206 с.

10. Щолокова О. П. Основні тенденції фортепіанної підготовки педагогамузиканта у вищих мистецьких закладах освіти // Topical issues of education : collective monograph. - Lisbon, Portugal : Pegasus Publishing, 2018. Р. 205-219.

11. Яранцева Н. Я. Художественная деятельность. Проблемы субъекта и объекта детерминации . Київ: Наукова думка. 1980. 244 с.

12. Pet'ko L. V. Formation of professionally oriented foreign language teaching environment in the conditions of university for students of specialties 023 «Fine Arts» and 022 «Design»// Economics, management, law:realities and perspectives: Collection of scientific articles. Psychology. Pedagogy and Education. - Les Editions L'Originale, Paris, France. 2016. P. 466-470.

13. Pet'ko L. V. Formation of professionally oriented foreign language teaching environment in the conditions of university for students of art specialties // Economics, 
management, law: problems of establishing and transformation: Collection of scientific articles. - Al-Ghurair Printing \& Publishing LLC, Dubai, UAE, 2016. P. 395-398.

14. Shcholokova O. P. Art and pedagogical designing as a means of improvement of music teacher's professional preparing // Economics, management, law: socio-economical aspects of development: Collection of scientific articles. Volum 2. - Edizioni Magi Roma, Italy. 2016. P. 265-268.

15. Shcholokova Olga. Modern Views on the problem Piano tone production in the musical pedagogical literature / Olga Shcholokova, Wang Xue // Intellectual Archive. Toronto : Shiny Word.Corp. (Canada). 2016. November/December. Vol. 5. No. 6. PP. 81-90. 


\title{
Temperamental Properties of Personality and its Importance in Organization of Sports Activity
}

\author{
Salikhov Timur Mansurovich \\ Master of Bukhara State University \\ Shoymardanova Mukhsina Rakhmatovna \\ Director of Bukhara School № 6
}

\begin{abstract}
Modern sport is also characterized by the fact that trainings and competitions take place against the background of significant emotional and mental loads, against the background of intense mental activity caused by the rapid pace of daily life, variety of external stimuli, etc.

Studies show that temperament significantly affects sports activity, determining first of all its dynamic side. Now, apparently, it can be said that types of nervous system and temperament are characterized by different ways of balancing the body with the environment. Temperament determines the character, dynamics, and often qualitative specificity of this adaptation activity - people with different temperament properties come to the same result in a peculiar way, sometimes with different degrees of time, power and energy.
\end{abstract}

Key words: personality, temperament, psychological characteristic of temperament types, temperament properties, sports activity, choleric, sanguine, phlegmatic, melancholic.

Success in sports, as in other types of human activity, depends on a number of conditions, including peculiarities of processes of education, upbringing, training, preparation for competitions. These processes should be built and implemented not only on the basis of general psycho-pedagogical patterns, but also taking into account specific, psychologically justified methods and techniques of pedagogical influence, methods of organization of activities adapted to individual properties of the nervous system and temperament of those engaged.

The importance of studying the problem of temperament is absolutely undeniable in connection with issues of sports pedagogy, psychology, theory and methodology of physical education and sports training. Development of this problem will contribute to scientific justification of individualization of processes of education, training, preparation and participation in competitions.

The mutual relationship of properties is also manifested in the fact that the qualitative characteristic of each individual property depends on all other properties inherent in a given type of temperament. For example, lack of support is known to depend on the strength of nerve processes and their balance. Instability, i.e. the prevalence of excitation force over braking force, is possible with different expressions of nervous system force. The lack of support of a 
person whose strong arousal prevails over equally strong braking is passionate rampant. The lack of support of a person whose weak excitement prevails over even weaker braking is hysterical instability.

Psychological characterization of temperament types. At present, the following main properties are taken into account for the psychological characteristic of temperament types:

1. Sensitivity. This property is judged by what is the least external force necessary for any mental response of a person. This includes the least physical force of the irritant necessary to create sensations (low threshold of sensations), the least degree of satisfaction of the need, causing joy or suffering in the person.

2. Reactivity. This property is characterized by the power with which people respond emotionally to external or internal influences (critical remark, offensive word, threat, sharp and unexpected sound). The brightest manifestations of reactivity are emotionality, impressiveness.

Activity. This property is expressed by the degree of human influence on the outside world, its ability to overcome obstacles in the implementation of the Goals. This includes targeting and perseverance in achieving the goal, focusing on long-term work, etc.

The ratio of reactivity to activity, i.e., on which human activity depends to a greater extent: on accidental external or internal circumstances (mood, desires) or on its goals, intentions, aspirations, beliefs.

Rate of reactions. It is determined by the rate of various mental reactions and processes (speed of movements, tempo of speech, resourceness, rate of memorization, speed of mind).

Plasticity - rigidity. This property is judged by how easily and flexibly a person adapts to external influences (plasticity) or, on the contrary, how inert and oblique his behavior, habits, judgements (rigidities) are.

7. Extraversion - an introversion. In determining this property, account is taken of: on what the reactions and activities of the person depend mainly - on external impressions arising at the moment (extroversion), or on images, perceptions and thoughts related to the past and the future (introversion).

Modern science does not yet have enough facts to fully characterize all types of temperament. However, in the psychological characteristic of the four traditional types of temperaments, a clear manifestation of these properties can be seen. 
Sanguine (based on a strong, balanced, mobile type of nervous system) favours sports that are associated with great mobility, activity, require courage. These individuals easily move from performing one kind of exercise to another, but are not sufficiently firm and focused, especially in one-of-a-kind (training) activities. When studying new exercises, they quickly grab the basis of the motion being studied, can perform it quite easily from the first attempt, but with errors; Do not like painstaking and long-term work to improve the technique of performing the exercise. Athletes of this type are quite workable, confident in themselves, sociable. Their sports results are stable and, as a rule, in competitions are higher than on trainings. Before the start such athletes are most often in a state of "combat readiness."

Choleric (strong, unbalanced, excitation-dominated, type of nervous system). The cholerictype athlete prefers highly emotional sports (basketball, sprint, jumping), intense and tempo movements. He willingly and with passion begins to engage in the chosen sport, but his enthusiasm quickly disappears. Such athletes are reluctant to perform long training work on strength and endurance, but are able to repeat a difficult and dangerous exercise multiple times if it has aroused interest. Their competitive results are not stable enough, there is a tendency to "pre-launch fever," which quite often does not allow them to fully realize their opportunities in competitions.

Phlegmatic (strong, balanced, inert type of nervous system). In sports this temperament manifests itself primarily in insufficient rapid development and conversion of motor skills, in slow reactions, in difficulties of switching from one activity to another. The skills and habits formed are characterized by great strength, conservativeness. Such athletes are very capable and resistant to external stimuli, give preference to non-fast, calm, uniform exercises, are prone to long-term and careful work of technique of individual exercises, painstaking work on development of any quality, etc. Not characterized by high sociability. As a rule, very persistent and persistent. Competitive results are stable. Before the start, they are most often on "alert."

Melancholic (weak type of nervous system). In sports activity melancholic is characterized by excessively high responsibility, highly developed muscle-motor sensitivity, thin tactical sensitivity, but insufficient performance and low resistance to external stimuli, high anxiety, generating uncertainty in the forces. These persons give preference to individual sports not connected with single combat and team wrestling, competitive results of them are unstable, as 
inherent high anxiety contributes to formation of a state of "starting apathy," which makes it difficult to achieve high results.

Thus, in each type of temperament, in each type of relationship of its properties there are such qualities which can significantly influence sports activity. Therefore it is necessary to know to what extent temperament favors and how much it prevents success at different levels of sports activity.

\title{
References
}

1. Asmolov A. G. Psikhologiia lichnosti. [Psychology of Personality] - M. 1990. - 367p.

2. Viatkin B.A. Rol' temperamenta v sportivnoi deiatel'nosti. [The role of temperament in sports activities.] - M.: Fizkul'tura i sport, 1978. - 134 p.

3. Krechmer E. Teoriia temperamentov. // Psikhologiia individual'nykh razlichii. Khrestomatiia. [Theory of temperaments. // Psychology of individual differences. An anthology] - M. 2000. - P. 19-24

4. Merlin V.S. Ocherk teorii temperamenta. [Essay on the theory of temperament.] - M., 1964. $-304 \mathrm{p}$.

5. Nebylitsyn V.D. Aktual'nye problemy differentsial'noi psikhofiziologii. // Psikhologiia individual'nykh razlichii. Khrestomatiia. [Actual problems of differential psychophysiology. // Psychology of individual differences. An anthology.] - M., 2000. - P. 667-677.

6. Il'in E.L. Psikhologiia fizicheskogo vospitaniia: uchebnik dlia institutov i fakul'tetov fizicheskoi kul'tury [Psychology of physical education: a textbook for institutes and faculties of physical education] / E.L. Il'in. - SPb.: publishing house RGPU im. A.I. Gertsena, 2000. $486 \mathrm{p}$.

Translation of the Title, Abstract and References to the Author's Language

\section{ТЕМПЕРАМЕНТАЛЬНЫЕ СВОЙСТВА ЛИЧНОСТИ И ЕЕ ЗНАЧЕНИЕ В ОРГАНИЗАЦИИ СПОРТИВНОЙ ДЕЯТЕЛЬНОСТИ}

\author{
Салихов Тимур Мансурович \\ Магистрант Бухарского государственного университета, \\ Шоймарданова Мухсина Рахматовна \\ Директор школь №6 г. Бухары
}

Аннотация: Современный спорт характеризуется также тем, что тренировки и соревнования проходят на фоне значительных эмоциональных и психических нагрузок, на фоне напряженной умственной деятельности, вызываемых стремительным темпом повседневной жизни, многообразием внешних раздражителей и т. п. 
Исследования показывают, что темперамент существенным образом влияет на спортивную деятельность, определяя прежде всего ее динамическую сторону. Сейчас, видимо, можно говорить о том, что типы нервной системы и темперамента характеризуются разными способами уравновешивания организма со средой. Темперамент определяет характер, динамику, а нередко и качественное своеобразие этой приспособительной деятельности - люди с различными свойствами темперамента приходят к одному и тому же результату своеобразным путем, порой с разной степенью затраты времени, сил и энергии.

Ключевые слова: личность, темперамент, психологическая характеристика типов темпераментов, свойства темперамента, спортивная деятельность, холерик, сангвиник, флегматик, меланхолик.

\section{References}

1. Асмолов А. Г. Психология личности. - М. 1990. - 367 с.

2. Вяткин Б.А. Роль темперамента в спортивной деятельности. - М.: Физкультура и спорт, 1978. - 134 с.

3. Кречмер Э. Теория темпераментов. // Психология индивидуальных различий. Хрестоматия. - М., 2000. - С.19-24

4. Мерлин В.С. Очерк теории темперамента. - М., 1964. - 304 с.

5. Небылицын В.Д. Актуальные проблемы дифференциальной психофизиологии. // Психология индивидуальных различий. Хрестоматия. - М., 2000. - 667-677.

6. Ильин Е.Л. Психология физического воспитания: учебник для институтов и факультетов физической культуры / Е.Л. Ильин. - СПб.: изд-во РГПУ им. А.И. Герцена, 2000. -486 c. 


\title{
Psychological Features of Formation of Emotional-Will Sphere of Athlete Personality
}

\author{
Shohrukh Salihov, \\ Bukhara State University, Uzbekistan \\ Malika Umidjanova \\ Bukhara State University, Uzbekistan
}

\begin{abstract}
Numerous data on the personality characteristics of athletes and non-athletes show significant differences in the levels of expression of a range of personality qualities. Inspection of big groups of athletes of high qualification shows that most characteristics of their personality are: high emotional stability, self-confidence, independence, independence, tendency to risk, self-checking, sociability.
\end{abstract}

Key words: personality, athlete, emotional-will sphere, aggressiveness, motivation, mental tension, motive of achievement.

At present the issue of "personal profile" of the athlete engaged in a certain sport is quite widely discussed. However, the analysis of personal features of athletes of the international class on sports did not confirm the hypothesis of "personal profile."

More productive was the approach of finding common manifestations of personality that lead to success in sports. Among such features should be: sensivity, emotional resistance, activity in overcoming obstacles.

According to American sports psychologists, the most common personal traits of athletes include:

- High level of aggressiveness (which is almost always under control of the athlete of high class),

- High level of achievement motivation,

- Extroversion and character hardness,

- authoritativeness,

- Emotional stability and self-control [1].

Aggression. In many sports, especially those where direct physical contact is allowed, various forms of controlled physical aggression are simply necessary. Research data show that high-class athletes engaged in these sports are not only more aggressive, but also tend to express their aggressive tendencies more freely than representatives of the so-called normal sample. At the same time aggressiveness is expressed in different ways in athletes engaged in different sports.

Motivation to achieve success in athletes. Motivation is a key variable of mastering sports skills, the ability to demonstrate them. Motivation is, first of all, a desire for success, high results in its activities. And if a person, seeks to achieve success, high results in activity, 
he has quite strong motivation of achievement. The main psychological feature of the motives that encourage a person to engage in sports is the sense of satisfaction caused by the activities of this sport and inseparable from the sports activity itself.

From the analysis of literary sources devoted to this problem, it follows that a special place in psychological support of sports activity is occupied by motivation, which encourages a person to engage in sports. The motivation unit forms needs, motives and goals of sports activity.

E.P. Ilin notes that at the initial stage the reasons for entering sports (regardless of activity, i.e. sports) may be:

aspiration to self-improvement;

The desire for self-expression and self-affirmation;

social installations;

Satisfaction of spiritual and material needs [2].

Each of the listed reasons has more or less efficiency for a particular athlete due to its value orientations.

American psychologist B. J. Cretti identifies:

Seeking and overcoming stress; He notes that fighting to overcome obstacles, to expose yourself to stress, to change circumstances and to achieve success is one of the strongest motives of sports activity;

commitment to excellence;

increase in the social status;

Need to be a member of sports team, group, part of team;

receiving material encouragement.

Y.Y. Palayma allocated two groups of the athletes differing for the dominating motive. In the first group, provisionally called "collectivists," social, moral motives are dominant. In the second group - "individualists" - the leading role is played by motives of self-affirmation, self-expression of personality. The former perform better in teams, and the latter, on the contrary, - in personal competitions.

The social motive is characterized by awareness of the social significance of sporting activity; Athletes with dominance of this motive set for themselves high perspective goals, they are passionate about sports activities. The motive of self-assertion is characterized by excessive orientation of athletes on evaluation of the sports results. [5]

The results of practical studies conducted by sports psychologists show that contact with opponents or partners is also an important motivational feature of the chosen sport. Three main types of contacts are distinguished: direct (in hockey, boxing, wrestling, rugby, etc.), mediated (volleyball, football, chess, basketball, tennis, etc.) and conditional (swimming, gymnastics, etc.) [3]

Activity in sports of the highest achievements is connected with high psychological tension caused by non-compliance of requirements of sports activity to opportunities of the athlete and aimed at elimination of this non-conformity. [4] In comparison with other activities, 
in sports high mental tension is fundamentally impossible, without it is impossible to exceed previous records. Therefore athletes at the stage of higher sports skill form not only motives of achievement of success, but also motives and motives of avoidance of failure. Any situation that actualizes in the athlete the motive of success, at the same time generates fears of failure. What motive arises depends on subjective estimates of the probability of achieving the goal, which are formed under the influence of competitive and life experience. Thus, a steadily dominant type of activity motivation is formed.

\section{References}

1. Beron R., Richardson D. Agressiia [Aggression] - SPb, Piter, 2000 - 352 p.

2. Il'in E.P. Psikhologiia fizicheskogo vospitaniia: Uchebnoe posobie dlia studentov pedagogicheskikh institutov. [Psychology of Physical Education: A Textbook for Students of Pedagogical Institutes.] / E.P Il'in - M.: Prosveshchenie, 1987. - 287 p.

3. Piloian R.A. Motivatsiia sportivnoi deiatel'nosti: [Motivation of sports activity:] /Piloian R.A. - M.: FiS, $1984-108$ p.

4. Singer R.N. Mify i real'nost'v psikhologii sporta: uchebnik dlia institutov fizicheskoi kul'tury. [Myths and reality in the psychology of sports: a textbook for institutes of physical education.] / R.N. Singer per. s angliiskogo / Predislovie A.V. Rodionova-M: Fizkul'tura i sport $1980 \mathrm{~s} .-152 \mathrm{p}$.

5. Stanbulova N.V. Psikhologiia sportivnoi kar'ery: uchebnik dlia institutov fizicheskoi kul'tury. [Psychology of a sports career: a textbook for institutes of physical education] / N.V. Stanbulova - M.: $2005-63$ p.

Translation of the Title, Abstract and References to the Author's Language

\section{ПСИХОЛОГИЧЕСКИЕ ОСОБЕННОСТИ ФОРМИРОВАНИЯ ЭМОЦИОНАЛЬНО-ВОЛЕВОЙ СФЕРЫ ЛИЧНОСТИ СПОРТСМЕНА Шохрух САЛИХОВ, Малика УМИДЖАНОВА Бухарский государственный университет, Узбекистан}

Многочисленные данные о личностных особенностях спортсменов и лии, не занимающихся спортом, свидетельствуют о существенных различиях в уровнях выраженности целого ряда личностных качеств. Обследование больших групп спортсменов высокой квалификации показывает, что наиболее характерными особенностями их личности являются: высокая эмоциональная устойчивость, уверенность в себе, независимость, самостоятельность, склонность к риску, самоконтроль, общительность.

Ключевые слова: личность, спортсмен, эмочионально-волевая сфера, агрессивность, мотивация, психическое напряжение, мотив достиюения. 


\section{Список литературы}

1. Бэрон Р., Ричардсон Д. Агрессия - СПб, Питер, 2000 - 352 c.

2. Ильин Е.П. Психология физического воспитания: Учебное пособие для студентов педагогических институтов./ Е.П Ильин - М.: Просвещение, 1987. -287 с.

3. Пилоян Р.А. Мотивация спортивной деятельности: /Пилоян Р.А. - М.: ФиС, 1984 $108 \mathrm{c}$.

4. Сингер Р.Н. Мифы и реальность в психологии спорта: учебник для институтов физической культуры./ Р.Н. Сингер пер. с английского / Предисловие А.В. Родионова М: Физкультура и спорт 1980 с. -152 с.

5. Станбулова Н.В. Психология спортивной карьеры: учебник для институтов физической культуры./ Н.В. Станбулова - М.: $2005-63$ с. 


\section{Manuscript Guidelines}

1. All submitted papers must contain the Title, Name of author(s), Affiliation (if any), Abstract and List of References (Literature) written in English. The Abstract must count not less than 100 and not more than 300 words and must be the good representation of your article. Optionally paper may also contain this information duplicated in another language.

2. Font faces. Arial, Times, Times New Roman, Courier New and Helvetica.

3. Language. You may use any language for your paper text, however English is MUCH preferable.

4. Title. Font size - 16, bold. Position - central alignment.

5. The author's name. Font size - 14, bold. Position - central alignment.

6. The affiliation (your University etc). Font size - 14, regular (not bold). Position - left alignment.

7. The word "Abstract". Font size - 12, bold-italics. Position - central alignment.

8. The text of the abstract. Font size - 10, regular (not bold).

9. The word "Keywords" (if any). Font size - 10, bold. Position - left alignment.

10. The text of keywords (if any). Font size - 10, regular (not bold). Position - left alignment.

11. Text of article. Font size - 14. Position - left alignment or fully justified. Line spacing - 1.5 lines.

12. The word "References" (if any). Font size - 12, bold-italics. Position - central alignment.

13. The text of References (if any). Font size - 12, regular (not bold).

In all other cases please use your own good judgment or contact our Editorial Board.

\section{Where to find us}

The "IntellectualArchive" is distributed to major libraries across Canada and the US, including

Library of Congress, USA (http://lccn.loc.gov/cn2013300046 ) ,

Library and Archives Canada

(http://collectionscanada.gc.ca/ourl/res.php?url_ver=Z39.88-2004\&url_tim=2012-09-

05T01\%3A46\%3A54Z\&url_ctx_fmt=info\%3Aofi\%2Ffmt\%3Akev\%3Amtx\%3Actx\&rft_dat=40904933\&r

fr_id=info\%3Asid\%2Fcollectionscanada.gc.ca\%3Aamicus\&lang=eng) and others.

The references to articles published in the "IntellectualArchive" are available in the

Google Scholar, (http://scholar.google.ca/scholar?q=\%22IntellectualArchive\%22 ),

Arxiv.org (http://search.arxiv.org:8081/?query=\%22Intellectual\%20Archive\%22\&in= ),

WorldCat.org (https://www.worldcat.org/search?q=n2\%3A1929-4700\&qt=advanced \&dblist=638 ) ,

Academia.edu

(http://www.academia.edu/15503799/Light_diffraction_experiments_that_confirm_the_STOE_model_and

_reject_all_other_models )

The National Research Council (Italy) (http://data.cnr.it/data/cnr/individuo/rivista/ID658222 )

Наукова бібліотека of the University named after Dragomanov, Ukraine

(http://enpuir.npu.edu.ua/handle/123456789/7974?mode=full )

Google.com (https://www.google.ca/\#q=site:IntellectualArchive.com ) thousands of links etc. 